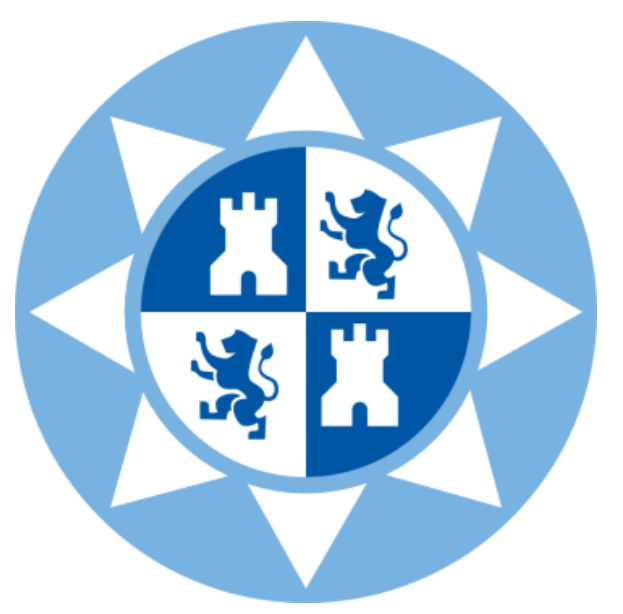

Universidad Politécnica de Cartagena

Department of Information and Communication Technologies

\title{
Analysis and Design of New Electronically Reconfigurable Periodic Leaky-Wave Antennas
}

(Doctoral Thesis)

\author{
Dissertation written by \\ Raúl Guzmán Quirós
}

\author{
Supervised by \\ Dr. José Luis Gómez Tornero
}

Cartagena, September 2014 



\section{Universidad}

Politécnica

de Cartagena

DT-16

\section{CONFORMIDAD DE SOLICITUD DEAUTORIZACIÓN DE DEPÓSITO DE TESIS DOCTORAL POR EL/LA DIRECTOR/A DE LA TESIS}

D. JOSÉ LUIS GÓMEZ TORNERO Director de la Tesis doctoral ANALYSIS AND DESIGN OF NEW ELECTRONICALLY RECONFIGURABLE PERIODIC LEAKYWAVE ANTENNAS

\section{INFORMA:}

Que la referida Tesis Doctoral, ha sido realizada por D/Da.RAÚL GUZMÁN QUIRÓS, dentro del programa de doctorado TECNOLOGÍAS DE LA INFORMACIÓN Y LAS COMUNICACIONES, dando mi conformidad para que sea presentada ante la Comisión de Doctorado para ser autorizado su depósito.

La rama de conocimiento en la que esta tesis ha sido desarrollada es:

Ciencias

Ciencias Sociales y Jurídicas

Ingeniería y Arquitectura

En Cartagena, a 5 de JUNIO de 2014

EL DIRECTOR DE LA TESIS

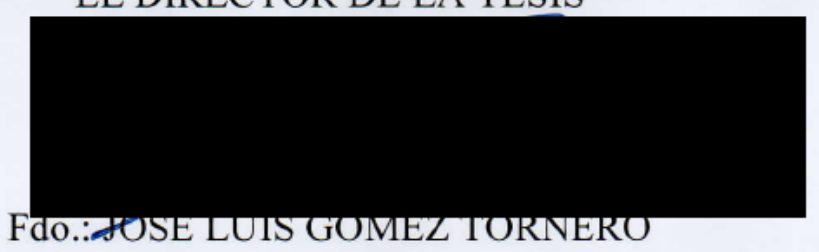





\section{CONFORMIDAD DE DEPÓSITO DE TESIS DOCTORAL POR LA COMISIÓN ACADÉMICA DEL PROGRAMA}

D. Fernando Daniel Quesada Pereira Presidente de la Comisión Académica del Programa TECNOLOGÍAS DE LA INFORMACIÓN Y LAS COMUNICACIONES.

\section{INFORMA:}

Que la Tesis Doctoral titulada, "ANALYSIS AND DESIGN OF NEW ELECTRONICALLY RECONFIGURABLE PERIODIC LEAKY-WAVE ANTENNAS”, ha sido realizada, dentro del mencionado programa de doctorado, por D. Raúl Guzmán Quirós,

bajo la dirección y supervisión del Dr. José Luis Gómez Tornero.

En reunión de la Comisión Académica de fecha 6 de Junio de 2014, visto que en la misma se acreditan los indicios de calidad correspondientes y la autorización del Director de la misma, se acordó dar la conformidad, con la finalidad de que sea autorizado su depósito por la Comisión de Doctorado.

La Rama de conocimiento por la que esta tesis ha sido desarrollada es:

$\square$ Ciencias

$\square$ Ciencias Sociales y Jurídicas

$\checkmark$ Ingeniería y Arquitectura

En Cartagena, a 6 de Junio de 2014

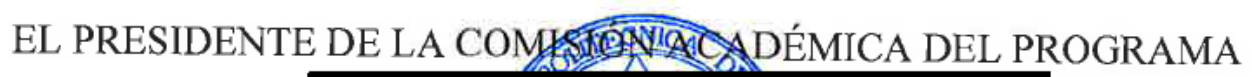

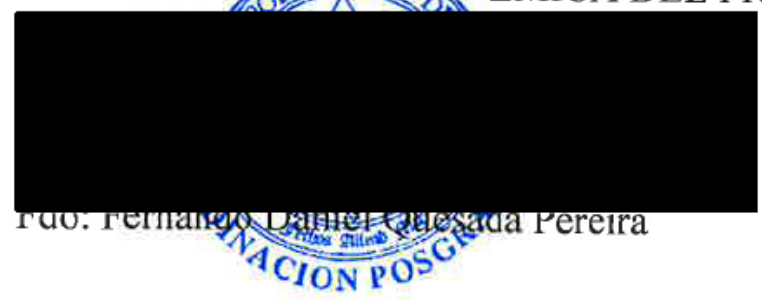

COMISIÓN DE DOCTORADO 

"Vivir no es sólo existir, sino existir y crear, saber gozar y sufrir y no dormir sin soñar.

Descansar, es empezar a morir."

Gregorio Marañón

A mis padres. 



\section{Contents}

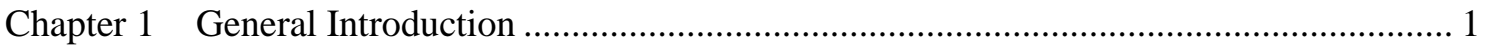

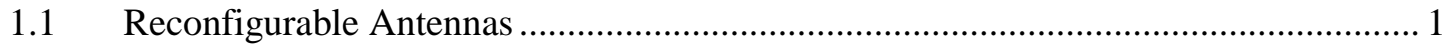

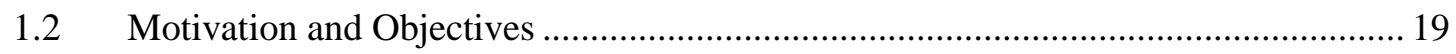

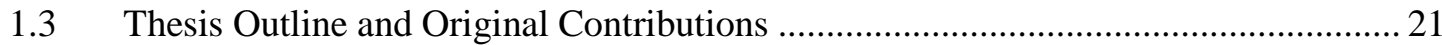

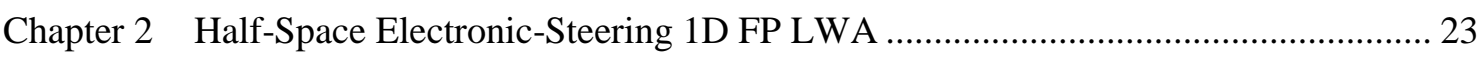

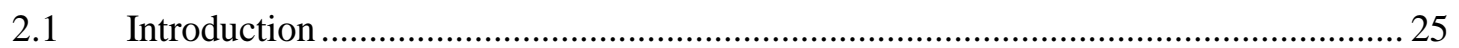

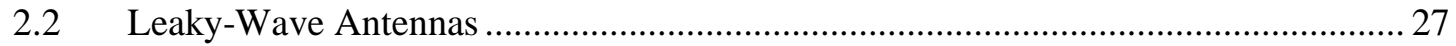

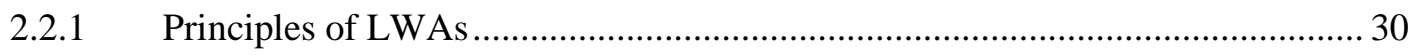

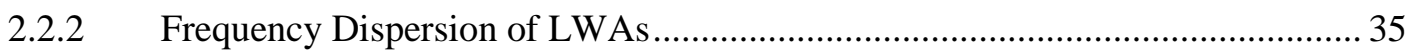

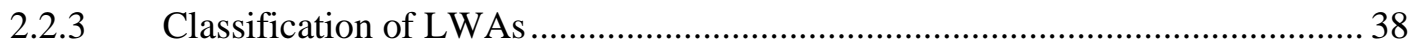

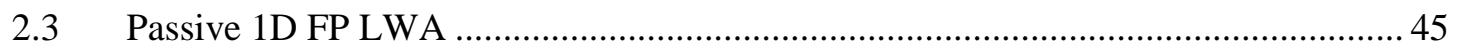

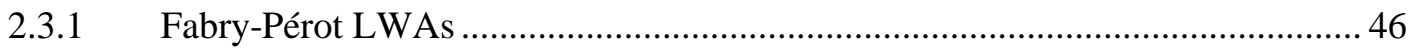

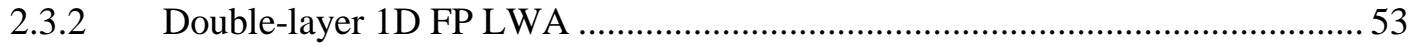

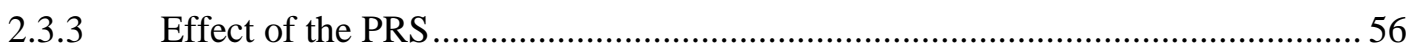

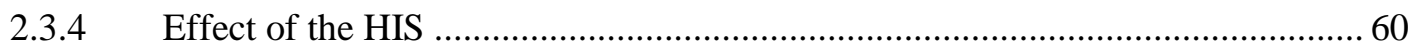

2.3.5 Control of the pointing angle and beamwidth in 1D FP LWAs ........................... 64

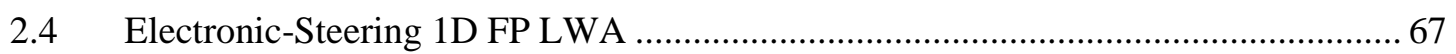

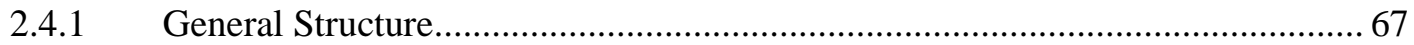

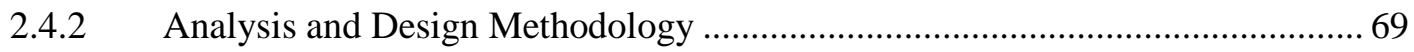

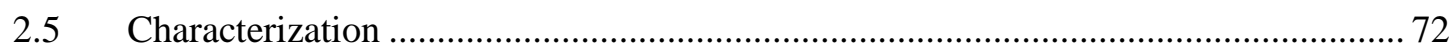

2.5.1 Transverse Equivalent Network ……............................................................ 73

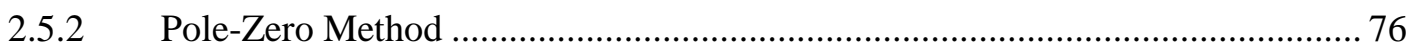

2.5.3 Design and Characterization of the Passive PRS …............................................ 80

2.5.4 Design and Characterization of the Tunable HIS …........................................... 84

2.6 Electronic Control of the Pointing Angle …….......................................................... 91

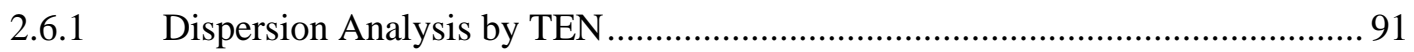

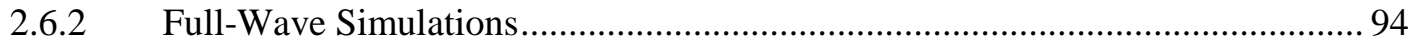


2.6.3 Optimization of the Dynamic Scanning Range .................................................. 97

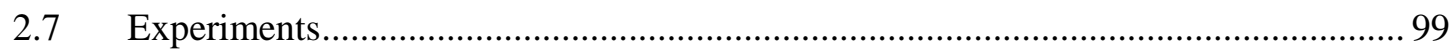

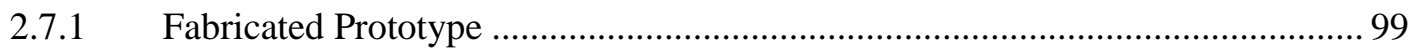

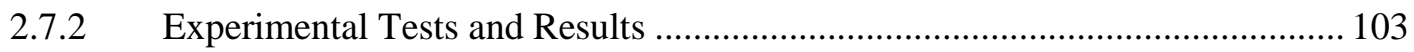

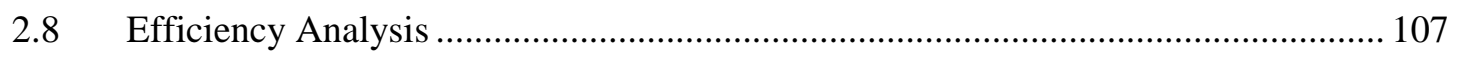

2.9 Analysis of the Scanning Pattern Bandwidth ....................................................... 111

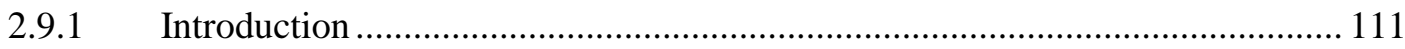

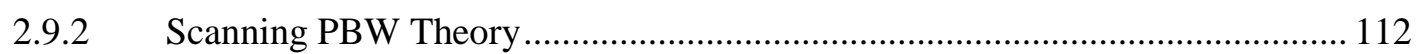

2.9.3 Analysis Example on the Electronic-Steering 1D FP LWA …......................... 114

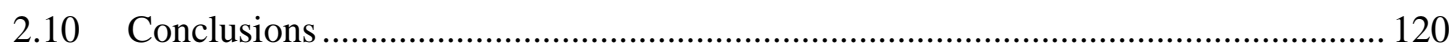

Chapter 3 Full-Space Electronic-Steering 1D FP LWA …........................................................ 123

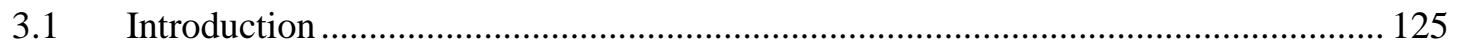

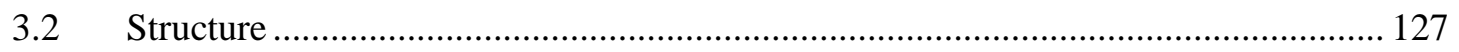

3.3 Dispersion Analysis and Defined Regions ............................................................ 128

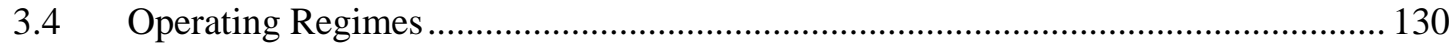

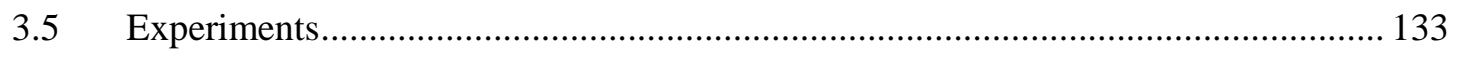

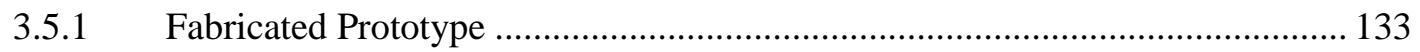

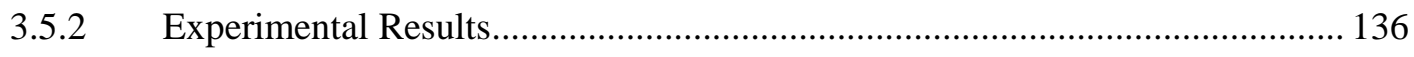

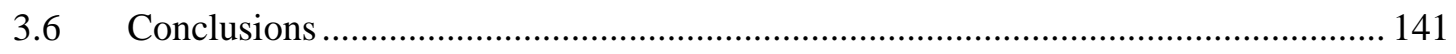

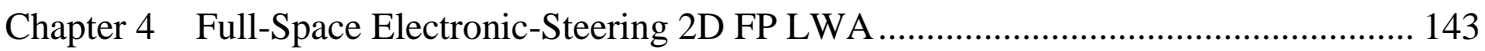

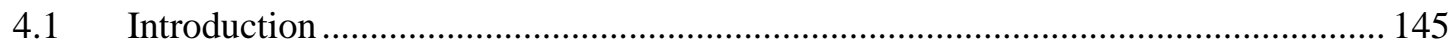

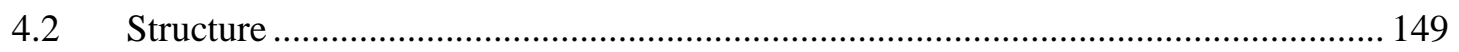

4.3 Dispersion Regions and Operating Regimes ....................................................... 152

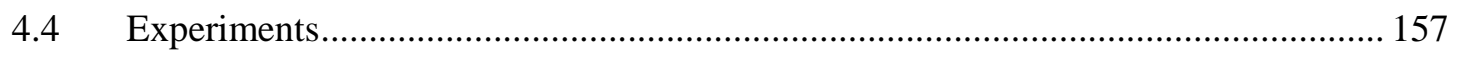

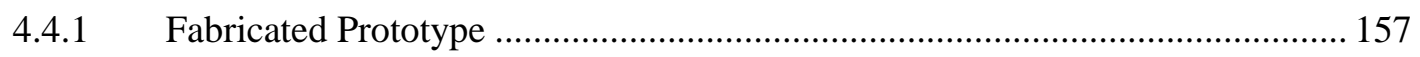

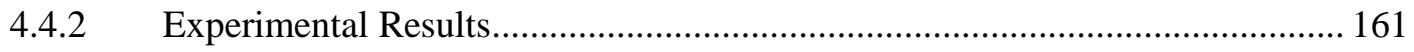

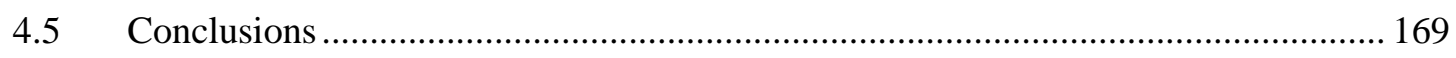

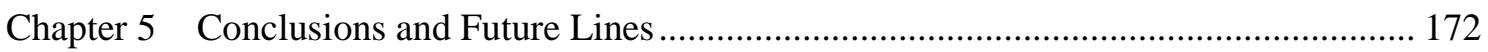

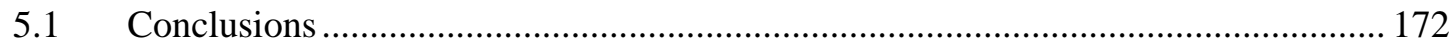




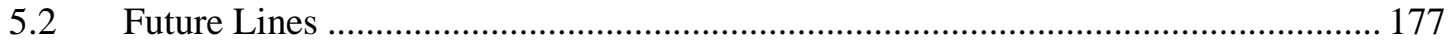

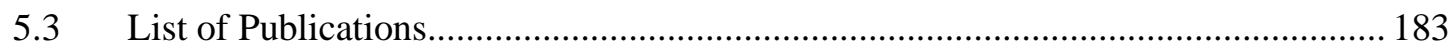

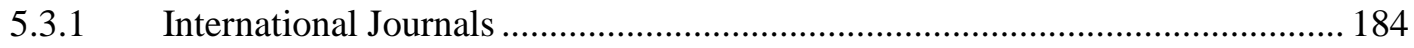

5.3.2 International Conference Proceedings.............................................................. 185

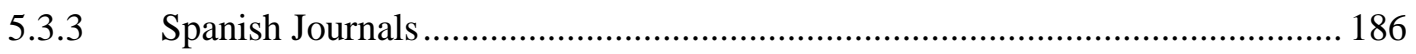

5.3.4 Spanish Conference Proceedings ………….................................................... 187

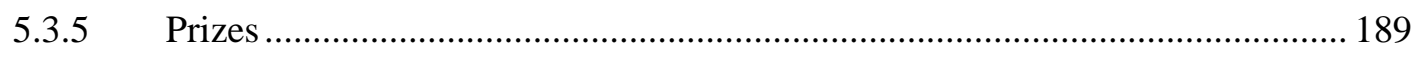

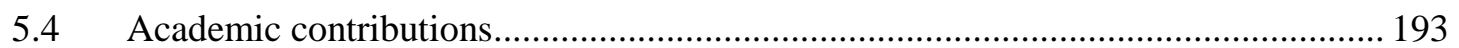

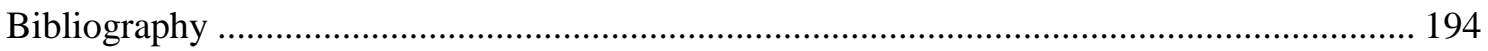

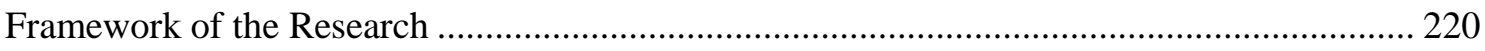

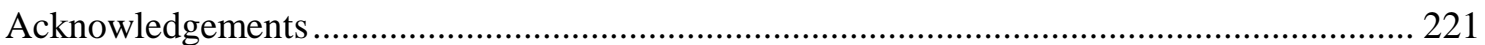




\section{List of Figures}

Fig. 1.1.1 (a) Photograph of a reconfigurable rectangular slot antenna in two different configurations (after [Gupta 2000]). (b) Fluidic frequency reconfigurable slot antenna (after [King 2013]). (c) Yagi antenna with varactor diodes for frequency reconfiguration (after [Cai 2012]). (d) Frequency and polarization agile microstrip patch antenna (after [Balcells 2010])... 4

Fig. 1.1.2 A seven-element circular array of reactively loaded parasitic dipoles for reconfigurable beam steering and beamforming (after [Harrington 1978])......

Fig. 1.1.3 Beam-steering phased array using phase-shifters at each radiating element (after [Hansen 1998]).

Fig. 1.1.4 (a) General scheme of a printed reflectarray antenna (after [Huang 2008]). (b) General scheme of a printed transmitarray antenna (after [Sazegar 2012]).

Fig. 1.1.5 (a) General scheme of a pixelled antenna (after [Pringle 2008]). Configurations of the switches to configure (b) bow-tie antenna and (c) Yagi antenna.

Fig. 1.1.6 (a) Depiction of a controllable plasma grid structure for a reconfigurable aperture: The grid structure is fabricated on top of the silicon wafer. (b) A cross-section of the plasma grid structure shows the layer interconnection (after [Fathy 2003]).

Fig. 1.1.7 (a) Top view and (b) Side view of a tunable textured surface. This feed can launch (c) forward leaky waves or (d) backward leaky waves (after [Sievenpiper 2005]).

Fig. 1.1.8 30-cell prototype of the voltage-controlled reconfigurable CRLH LWA structure with a magnified view of the unit cell. The E-field polarization is along the $x$-direction (after [Lim 2004-II]).

Fig. 1.1.9 Cutaway drawing of the frequency reconfigurable FP LWA. One quarter of the PRS material has been removed to show the details of the antenna more clearly (after [Weily 2008]).

Fig. 1.1.10 (a) Electronically tunable metasurface reflector. (b) Measured gain patterns in the Eplane $\left(\phi=90^{\circ}\right)$ at $7.9 \mathrm{GHz}$ for $\delta \mathrm{V}=0 \mathrm{~V}, \delta \mathrm{V}=0.2 \mathrm{~V}$ and $\delta \mathrm{V}=0.3 \mathrm{~V}$. The steering of the antenna's radiated beam can be clearly observed with a positive steering angle for positive bias and negative one for a negative bias (after [Burokur 2013]).

Fig. 1.1.11 Proposed array-fed PRS antenna with independent dynamic beamwidth control and beam-scanning in the H-plane (XZ-plane). All physical dimensions are in millimeters (after [Debogovic 2014-II]).

Fig. 2.2.1 Types of travelling wave antennas. (a) Leaky-wave antenna. (b) Surface-wave antenna. (c) Slot-waveguide antenna (Fig. 2.18 in [Gómez 2006-I]). 28

Fig. 2.2.2 Scheme of generic one-dimensional (1D) dielectric-filled guiding structure which supports a propagating (a) leaky wave and (b) a surface wave. 
Fig. 2.2.3 Example of classical frequency-dispersion diagram in a uniform 1D dielectric-filled LWA. Leaky-mode propagation regimes. 35

Fig. 2.2.4 Comparison between dispersion in a partly dielectric-filled waveguide LWA and an air-filled empty waveguide LWA (Fig. 11.3 in [Oliner 2007]) 36

Fig. 2.2.5 Scheme of 1D LWA excited from one end (Fig. 3.1.6 in [García-Vigueras 2012-II]).

Fig. 2.2.6 Normalized radiation pattern in $\mathrm{dB}$ of $1 \mathrm{D}$ LWA with dimensions: $\mathrm{a}=0.5 \lambda_{0}, L_{A}=6 \lambda_{0}$, fed at one end $\left(k_{y} / k_{0}=0.6+j 0.01\right)$. (a) H-plane $(Y Z)$, (b) E-plane (XZ) (Fig. 3.1.7 in [GarcíaVigueras 2012-II]).

Fig. 2.2.7 Scheme of 2D LWA excited from the center (Fig. 3.1.10 in [García-Vigueras 2012II]).

Fig. 2.2.8 Illustration of the type of beams produced by a 2D FP LWA: (a) a broadside pencil beam and (b) a symmetric conical beam at an angle $\theta_{0}$ (Fig. 7.5 in [Jackson 2008]).

Fig. 2.2.9 Pencil beam pointing at broadside produced by a 2D LWA $\left(L_{A}=6 \lambda_{0}\right)\left(k_{y} / k_{0}=\right.$ $0.1+\mathrm{j} 0.1$ ). Normalized radiation pattern in $\mathrm{dB}$ of (a) H-plane (YZ), (b) E-plane (XZ) (Fig. 3.1.11 in [García-Vigueras 2012-II]).

Fig. 2.2.10 Conical beam produced by a 2D LWA $\left(L_{A}=6 \lambda_{0}\right)\left(k_{y} / k_{0}=0.39+\mathrm{j} 0.015\right)$. Normalized radiation pattern in $\mathrm{dB}$ of 2D LWA $\left(L_{A}=6 \lambda_{0}\right)$. (a) H-plane (YZ), (b) E-plane (XZ) (Fig. 3.1.12 in [García-Vigueras 2012-II]) 40

Fig. 2.2.11 Example of uniform LWAs: (a) Slot waveguide ([Hansen 1940]), (b) Higher order mode microstrip LWA ([Menzel 1979]) and (d) 2D FP LWA with a stack of dielectric layers ([Jackson 1993]).

Fig. 2.2.12 Example of periodic LWAs: (a) Holey waveguide ([Hines 1957]), (b) Dielectric rod with metal strips, (c) LWA CRLH microstrip line ([Caloz 2005]), (d) Double-layer (PRS-HIS) 1D FP LWA ([García-Vigueras 2012-II]) and (e) 2D FP LWA composed of a dielectric slab with an array of patches ([Zhao 2005-II]). 42

Fig. 2.2.13 Dispersion in a periodic LWA (substrate with permittivity $\varepsilon_{r}$ ) (Fig. 3.1.16 in [GarcíaVigueras 2012-II]). 44

Fig. 2.3.1 Schematic diagram of a classical Fabry-Pérot LWA excited from the centre. 46 Fig. 2.3.2 (a) Original antenna scheme of multiple reflections between reflector and PRS and (b) PRS-loaded designed antennas (Figs. 1 and 7 in [Trentini 1956]).

Fig. 2.3.3 (a) Scheme of a patch-based free-standing FSS. (b) Some typical FSS unit cell geometries: (1) square patch, (2) dipole, (3) circular patch, (4) cross dipole, (5) Jerusalem cross, (6) square loop, (7) circular loop, (8) square aperture (Figs. 1 and 5 in [Mittra 1988]). 49 Fig. 2.3.4 (a) Original configuration of the passive 1D Fabry-Pérot LWA and (b) Transverse Equivalent Network proposed in [García-Vigueras 2012-II]. ( $a=H=11 \mathrm{~mm}, S=5 \mathrm{~mm}, D=1.13 \mathrm{~mm}$, 
$\varepsilon_{P R S}=\varepsilon_{H I S}=2.2, L_{P R S}=9 \mathrm{~mm}, L_{H I S}=9 \mathrm{~mm}, P=1.5 \mathrm{~mm}, Q=0.5 \mathrm{~mm}$ ) (Fig. 3.2 .1 in [García-Vigueras 2012-II]) .53

Fig. 2.3 .5 (a) Dispersion of natural modes in the 1D FP LWA ( $\left.L_{P R S}=L_{H I S}=10 \mathrm{~mm}\right)$ (b) Transverse electric fields in the cross-section of the LWA for each mode (Fig. 3.2.2 in [GarcíaVigueras 2012-II]). 54

Fig. 2.3.6 Leaky-mode frequency dispersion curves for the 1D FP LWA in Fig. 2.3.4(a) for different values of $L_{P R S}\left(L_{H I S}=9 \mathrm{~mm}\right)$ (Fig. 3.2.3 in [García-Vigueras 2012-II]). 56

Fig. 2.3.7 (a) Reflection coefficient (magnitude and phase) of a dipole-based PRS for different values of the dipoles length $\left(L_{P R S}\right)$. (b) Dispersion diagram of the PRS-HIS FP LWA (Fig. 3.2.5 in [García-Vigueras 2012-II]).

Fig. 2.3.8 (a) Reflection coefficient of the PRS and (b) Dispersion diagram of the TE leaky mode as a function of $L_{P R S}$ at fixed frequency of operation of $15 \mathrm{GHz}$ (Fig. 3.2.6 in [GarcíaVigueras 2012-II]). .58

Fig. 2.3.9 (a) Transversal and (b) longitudinal electric field of the TE leaky mode for different configurations of $L_{P R S}(15 \mathrm{GHz})$ (Fig. 3.2.7 in [García-Vigueras 2012-II]). 59

Fig. 2.3.10 Leaky-mode dispersion curves of the 1D FP LWA in Fig. 2.3.4(a) for different lengths of the HIS dipoles $\left(L_{H I S}\right)$. (a), (b) Dispersion with frequency, (c) Dispersion with $L_{H I S}$ at 15GHz (Fig. 3.2.8 in [García-Vigueras 2012-II]).

Fig. 2.3.11 (a) Reflection coefficient of the dipole-based HIS as a function of $L_{H I S}$ and (b) Electric field pattern in the transversal section of the FP LWA in Fig. 2.3.4(a) obtained from the TEN at $15 \mathrm{GHz}$ (Fig. 3.2.9 in [García-Vigueras 2012-II])

Fig. 2.3.12 Longitudinal electric field of the leaky modes for different configurations of $L_{H I S}$ at $15 \mathrm{GHz}$ (Fig. 3.2.10 in [García-Vigueras 2012-II]).

Fig. 2.3.13 General relation between the pointing angle and radiation rate of a leaky-wave (Fig. 3.2.11 in [García-Vigueras 2012-II]).

Fig. 2.3.14 2D contour curve plots of $\theta_{R A D}$ and $\alpha / k_{0}$ obtained at $15 \mathrm{GHz}$ (Fig. 3.3.1 in [GarcíaVigueras 2012-II]).

Fig. 2.3.15 Theoretical and measured normalized radiation patterns for the LWAs in Table 2.3.1 (a) showing the control of the beamwidth $(\Delta \theta)$ and (b) showing the control of the pointing angle $\left(\theta_{R A D}\right)$ (Fig. 3.3.4 and Fig.3.3.5 in [García-Vigueras 2012-II]).

Fig. 2.4.1 a) Scheme of the proposed reconfigurable 1D FP LWA b) Detailed scheme of the tunable high impedance surface unit cell $\left(a=25 \mathrm{~mm}, H=25.2 \mathrm{~mm}, L=13.6 \mathrm{~mm}, D_{H I S}=1.524 \mathrm{~mm}\right.$, $\varepsilon_{r H I S}=3, P_{H I S}=30 \mathrm{~mm}, Q_{H I S}=14 \mathrm{~mm}, L_{H I S}=17 \mathrm{~mm}, g=1 \mathrm{~mm}, D_{P R S}=0.8 \mathrm{~mm}, \varepsilon_{r P R S}=4.4, P_{P R S}=20 \mathrm{~mm}$, $\left.Q_{P R S}=18 \mathrm{~mm}, L_{P R S}=22 \mathrm{~mm}\right)$.

Fig. 2.5.1 Transverse Equivalent Network (TEN) of the reconfigurable 1D FP LWA 73 
Fig. 2.5.2 Compared scenarios to obtain the poles and zeros of the $Y_{F S S}$ for a TE incidence. TE plane wave impinging with incident angle $\left(\theta_{i}\right)$ on (a) an infinite two-dimensional FSS of

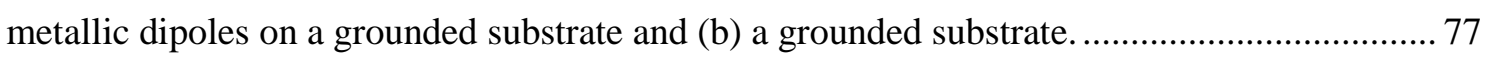

Fig. 2.5.3 Phase of the reflection coefficient under normal incidence $\left(\theta_{i}=0\right)$. ........................... 78 Fig. 2.5.4 (a) Simulation set-up to perform the plane wave incidence analysis on the PRS unit cell (Periodic Boundary Conditions (PBCs) are considered in the $\pm x$ and $\pm y$ directions). (b) Transmission line analyzed to extract the PRS admittance from the full-wave simulations. ..... 80 Fig. 2.5.5 Variation of (a) zeros and (b) poles of $Y_{P R S}$ with respect to the angle of incidence $\left(\theta_{i}\right)$.

Fig. 2.5.6 Reflection coefficient of the PRS (magnitude and phase) seen by a plane wave for different incidence angles as a function of $L_{P R S}$ at $5.6 \mathrm{GHz}$. 83

Fig. 2.5.7 Typical performance $V_{R}$ vs $C_{j}$ of Aeroflex Metelics MGV 125-08-0805-2 ([Aeroflex 2005]). 84

Fig. 2.5.8 (a) Configuration of the HIS unit cell employed to reconfigure the pointing angle of the reconfigurable antenna (Fig.3 in [Weily 2008]) and (b) Equivalent circuit of the varactor diode used to simulate the performance of the tunable HIS unit cell $\left(C_{p}=0.06 \mathrm{pF}, C_{j}=0.06 \mathrm{pF}\right.$ to 0.6pF) (Fig.4 in [Weily 2008])..... .85

Fig. 2.5.9 (a) Simulation set-up to perform the plane wave incidence analysis on the tunable HIS unit cell (PBCs are considered in the $\pm x$ and $\pm y$ directions). (b) Transmission line analyzed to extract the HIS admittance from the full-wave simulations. 87

Fig. 2.5.10 Variation of (a) zeros and (b) poles of $Y_{H I S}$ with respect to the angle of incidence $\left(\theta_{i}\right)$. 88

Fig. 2.5.11 Reflection phase of the tunable HIS seen by an incident plane wave for different incidence angles (a) as a function of frequency for different values of $C_{j}$ and (b) as a function of $C_{j}$ at $5.6 \mathrm{GHz}$. 89

Fig. 2.6.1 Leaky-mode dispersion curves as a function of frequency for different cases of $C_{j}$ $\left(L_{P R S}=22 \mathrm{~mm}\right)$ a) Normalized phase constant $\left(\beta_{y} / k_{0}\right)$ and b) Normalized leakage rate $\left(\alpha_{y} / k_{0}\right) \ldots . . .92$ Fig. 2.6.2 Leaky-mode dispersion curves as a function of $C_{j}$ for a frequency of $5.6 \mathrm{GHz}$. 92 Fig. 2.6.3 Leaky-mode electric-field pattern at the transverse section of the reconfigurable FP PRS-HIS cavity at $5.6 \mathrm{GHz}$ for different values of $C_{j}$. Results obtained by FEM ([CST 2010]). 94 Fig. 2.6.4 Leaky-mode longitudinal electric-field distribution inside the reconfigurable FP PRSHIS cavity at $5.6 \mathrm{GHz}$ for different values of $C_{j}$. Results obtained by FEM ([CST 2010]). .95

Fig. 2.6.5 Computed H-plane normalized radiation patterns for the proposed reconfigurable LWA at $5.6 \mathrm{GHz}$ for different $C_{j}$ for an antenna length $L_{A}=5.2 \lambda_{0}$. Theoretical array factor (solidlines) and simulations (dot-lines) represents the radiation patterns obtained from theoretical Array Factor approach. .95 
Fig. 2.6.6 Leaky-mode scanning-angle as a function of $C_{j}$ for different values of the Fabry-Perot cavity height, $H$, at $5.6 \mathrm{GHz}$. 97

Fig. 2.7.1 (a) 3D Model of the final design of reconfigurable 1D FP LWA ([CST 2010]) and (b) Photograph of the manufactured reconfigurable LWA prototype. 99

Fig. 2.7.2 Photographs of (a) Manufactured reconfigurable LWA prototype, (b) Tunable HIS phase agile cell, (c) Radiation pattern measurement experimental set-up and (d) S parameters measurements set-up.

Fig. 2.7.3 Coaxial-Waveguide transition scheme ([CST 2010]). (a) Perspective view of the 3D

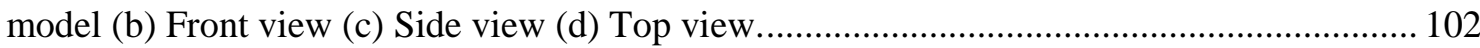

Fig. 2.7.4 Measured S parameters vs frequency for different $V_{R}$ a) $S_{11}$ b) $S_{21} \ldots \ldots \ldots \ldots \ldots \ldots \ldots \ldots . . . . . . . . . . .103$

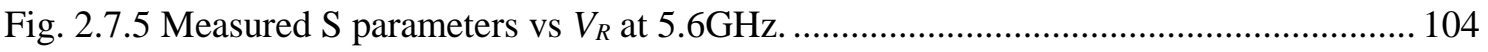

Fig. 2.7.6 Radiation response of reconfigurable LWA at $5.6 \mathrm{GHz}$ vs $V_{R}$ (a) Measured radiation patterns, (b) Pointing angle scanning, (c) Comparison between measured and theoretical (TRM) radiation patterns.

Fig. 2.8.1 Results at 5.6GHz vs $V_{R}$ (a) Measured gain, directivity and total efficiency, (b) Estimated efficiencies and (c) Simulated reflection coefficient (magnitude and phase) given by the tunable HIS (taken into account losses) as a function of the operating point of the antenna (given by simulations). 109

Fig. 2.9.1 Pattern bandwidth definition from the frequency scanning of a LWA 111

Fig. 2.9.2 (a) Normalized leaky-mode dispersion curves $\left(\beta / k_{0}\right.$ and $\left.\alpha / k_{0}\right)$ obtained from TEN for the half-space electronic scanning 1D FP LWA and (b) Scanning angle $\left(\theta_{R}\right)$ and scanning ratio (SR) as a function of frequency for different $V_{R}$ cases. (c) Scanning response and frequency scanning ratio as a function of $V_{R}$ at the fixed design frequency $f_{0}=5.6 \mathrm{GHz}$.

Fig. 2.9.3 Fractional pattern bandwidth (FPBW) as a function of scanning angle $\left(\theta_{R}\right)$ and antenna aperture length $\left(L_{A}\right)$ (theoretical lossless case).

Fig. 2.9.4 Comparison of Fractional PBW as a function of operating scanning point $\left(\theta_{R}\right)$ obtained from theory, ideal lossless simulations and lossy simulations.

Fig. 2.9.5 a) Simulated Realized Gain patterns at $f_{1}, f_{0}$ and $f_{2}$ for different scanning configurations b) Variation of the realized gain (simulated from final prototype) for each case as a function of frequency (values normalized w.r.t. maximum gain measured at central design frequency $f_{0}=5.6 \mathrm{GHz}$ for each respective case).

Fig. 3.2.1 Scheme of the proposed full-space steering 1D FP LWA. Same dimensions of original 1D FP LWA are considered $\left(a=25 \mathrm{~mm}, H=25.2 \mathrm{~mm}, L=13.6 \mathrm{~mm}, D_{H I S}=1.524 \mathrm{~mm}\right.$, $\varepsilon_{r H I S}=3, P_{H I S}=30 \mathrm{~mm}, Q_{H I S}=14 \mathrm{~mm}, L_{H I S}=17 \mathrm{~mm}, g=1 \mathrm{~mm}, D_{P R S}=0.8 \mathrm{~mm}, \varepsilon_{r P R S}=4.4, P_{P R S}=20 \mathrm{~mm}$, $\left.Q_{P R S}=18 \mathrm{~mm}, L_{P R S}=22 \mathrm{~mm}\right)$.

Fig. 3.3.1 Defined regions for the leaky-mode dispersion curves obtained for the half-space electronic-steering 1D FP LWA as a function of $C_{j}$ at $5.5 \mathrm{GHz}$. 128 
Fig. 3.4.1 Electric field distribution along the centrally excited reconfigurable 1D FP LWA obtained by FEM ([CST 2010]) for the three operating regimes: (a) Backward scanning (b) Forward scanning, (c) Broadside radiation

Fig. 3.4.2 Normalized H-plane radiation patterns obtained by FEM ([CST 2010]) at 5.5GHz for different $\left(C_{j L}, C_{j R}\right)$ operating configurations: (a) Backward scanning (b) Forward scanning, (c) Broadside radiation.

Fig. 3.5.1 (a) 3D Model of the final design of reconfigurable full-space electronic-steering 1D FP LWA excited from the center of the waveguide ([CST 2010]) and (b) Manufactured fullspace scanning 1D FP LWA prototype $\left(L_{A}=5 \lambda_{0}\right)$. 134

Fig. 3.5.2 Central coaxial feeder model ([CST 2010]). (a) Side view, (b) Front view and (c) Top view.

Fig. 3.5.3 Measured and simulated (FEM) scanning response for the full-space electronicsteering 1D FP LWA prototype at $5.5 \mathrm{GHz}$. 136

Fig. 3.5.4 Measured gain patterns for the full-space scanned 1D FP LWA at 5.5GHz for the three operating configurations.

Fig. 3.5.5 Measured and simulated S-parameters for the full-space scanned 1D FP LWA at 5.5GHz. a) Input matching $S_{11}$ b) Transmission $S_{21}, S_{31}$.

Fig. 4.1.1 (a) Schematic of the recent approach to simpler 2D scanning, involving a linear phased array of leaky-wave line sources, with frequency scanning in elevation and phase scanning in azimuth (Fig. 11.28 in [Oliner 2007]). (b) Generic pencil-beam form produced by the LWA phased array 146

Fig. 4.2.1 (a) 3D model of the full-space electronic-steering 2D FP LWA ([CST 2010]). Antenna aperture of $A=5.4 \lambda_{0} \times 5.4 \lambda_{0}$. (b) Top view of the 2D tunable HIS (with bias network included) divided in four independently biased sectors $\left(\mathrm{S}_{\mathrm{A}}, \mathrm{S}_{\mathrm{B}}, \mathrm{S}_{\mathrm{C}}, \mathrm{S}_{\mathrm{D}}\right)$ and (c) Top view of the 2D PRS.

Fig. 4.2.2 (a) Single unit-cell of the periodic 2D FP structure, (b) Transverse Equivalent Network for analyzing TE and TM leaky modes. ( $H=27 \mathrm{~mm}, D_{H I S}=1.524 \mathrm{~mm}, \varepsilon_{r H I S}=3$, $\left.P_{x}=P_{y}=30 \mathrm{~mm}, L_{H I S}=Q_{H I S}=17 \mathrm{~mm}, g=1 \mathrm{~mm}, L_{P R S}=Q_{P R S}=14.5 \mathrm{~mm}, D_{P R S}=0.8 \mathrm{~mm}, \varepsilon_{r P R S}=4.4\right) \ldots \ldots .150$ Fig. 4.3.1 (a) TE and (b) TM dispersion curves as function of $C_{j}$ at $5.5 \mathrm{GHz}$. 153 Fig. 4.3.2 (a) Near fields inside the 2D FP cavity $(z=H / 2)$ for several configurations of the sectors and (b) Associated radiation pattern in U-V coordinates (represented elevation $(\theta)$ range $\left.\theta=\left[0^{\circ}, 45^{\circ}\right]\right)$.

Fig. 4.3.3 (a) Normalized radiation patterns at $5.5 \mathrm{GHz}$ obtained from simulations (FEM ([CST 2010])). (a) TM leaky-mode scan (E-plane) at $\phi=0^{\circ}$, (b) TE leaky-mode scan (H-plane) at $\phi=90^{\circ}$, (c) Hybrid leaky-mode scan (EH-plane) at $\phi=45^{\circ}$, (d) Broadside radiation (four cutting planes). 
Fig. 4.4.1 Photographs of manufactured tunable HIS: (a) front view and (b) rear view. Photographs of the manufactured PRS: (c) front view and (d) rear view. 158

Fig. 4.4.2 Typical performance $V_{R}$ vs $C_{j}$ of Aeroflex Metelics MGV 125-20-0805-2 ([Aeroflex 2005]). 159

Fig. 4.4.3 Schematic of the stacked patch antenna employed for exciting the 2D FP cavity. (a) Side view (XZ plane), (b) Side view (YZ plane)

Fig. 4.4.4 Photographs of (a) S parameters measurement set-up and (b) Radiation pattern measurements set-up. 161

Fig. 4.4.5 Measured radiation patterns (normalized) obtained from the reconfigurable 2D FP LWA prototype at $5.5 \mathrm{GHz}$ for TM scan configurations at (a) $\phi=180^{\circ}$, (b) $\phi=0^{\circ}$ (E-Plane, $X Z$ ), for TE scan configurations at (c) $\phi=270^{\circ}$, (d) $\phi=90^{\circ}$ (H-Plane, YZ), for Hybrid TE/TM scan configurations at (e) $\phi=225^{\circ}$, (f) $\phi=245^{\circ}$, (g) $\phi=135^{\circ}$, (h) $\phi=315^{\circ}$ (diagonal planes).

Fig. 4.4.6 Broadside configuration for optimum operating point $\left(S_{A}=S_{B}=S_{C}=S_{D}=18.49 \mathrm{~V}\right)$.

Fig. 4.4.7 Measured and simulated (FEM, ([CST 2010])) scanning angle for different configurations at (a) TM regime, (b) TE regime and (c) Hybrid regime. 164 Fig. 4.4.8 Maximum Directivity (Simulated ([CST 2010])) and Realized Gain (simulated and measured) as function of the scanning angle (5.5GHz) for (a) TM scan, (b) TE scan, (c) Hybrid. 166

Fig. 4.4.9 Measured and simulated input matching $S_{11}$ for the Reconfigurable 2D FP LWA at 5.5GHz: (a) TM scan, (b) TE scan, (c) Hybrid scan. 167

Fig. 4.4.10 Measured realized gain as a function of the operating tuning voltage $\left(V_{R}\right)$ for Broadside configuration at $5.5 \mathrm{GHz}$.

Fig. 5.2.1 (a) Top view of the beam-steering 2D FP LWA. $S_{A}$ and $S_{B}$ are independently tuned at different points in the scanning region to control the wave vector of the hybrid (TE/TM) leaky mode in azimuth and elevation. (b) Associated aperture field for three configurations of the adjacent sectors ([CST 2010]) 179

Fig. 5.2.2 Schematic of proposed reconfigurable 1D FP LWA in SIW technology. (a) 3D model and cross section. (b) Front and rear views of the structure and defined unit cell (Teflon substrates with $\varepsilon_{l}=2.2$ and thicknesses $h_{l}=3.17 \mathrm{~mm}$ and $h_{2}=0.127 \mathrm{~mm}$ are used for the top and bottom layers, respectively).

Fig. 5.2.3 Normalized dispersion curves $\left(\beta / k_{0}\right.$ and $\left.\alpha / k_{0}\right)$ as a function of $C_{j H I S}$ for different $C_{j P R S}$ values, operating at a fixed frequency of $5.5 \mathrm{GHz}$. 181

Fig. 5.2.4 Theoretical radiation patterns (normalized directivity in $\mathrm{dB}$ ) obtained for different $C_{j H I S} \& C_{j P R S}\left(\beta / k_{0} \& \alpha / k_{0}\right)$ configurations operating at $5.5 \mathrm{GHz}$. 181 


\section{List of Tables}

Table 2.3.1 Electrical and geometrical parameters of the LWAs designed at $15 \mathrm{GHz}$ (Table.

3.3.1 in [García-Vigueras 2012-II]). 65

Table 2.7.1 Dimensions of the optimized coaxial-waveguide feeding network. 102

Table 2.7.2 Bias voltage and pointing angle for three operating points of the reconfigurable 1D FP LWA. 106

Table 2.9.1 Calculated Scanning Ratio for three scanning angles $\left(\theta_{R A D}\right)$ of the reconfigurable FP

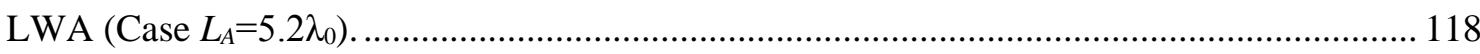

Table 3.5.1 Dimensions of the optimized coaxial-waveguide feeding network. 135

Table 4.4.1. Dimensions of the optimized stacked-patch antenna to excite the reconfigurable 2D FP LWA. 160

Table 4.4.2 Bias voltage, $S_{11}$, Realized Gain and Directivity (FEM and measured) for Broadside configuration at $5.5 \mathrm{GHz}$ at $18.49 \mathrm{~V}\left(C_{j}=0.1 \mathrm{pF}\right)$. 168

Table 5.2.1. Radiation angle $\theta_{R A D}$ and $-10 \mathrm{~dB}$ beamwidth $\left(\Delta \theta_{-10 d B}\right)$ values obtained for each $\left(C_{j H I S}, C_{j P R S}\right)$ configuration (Antenna aperture length considered: $\left.L_{A}=12 \lambda_{0}\right)$. 181 



\section{Chapter 1 General Introduction}

\subsection{Reconfigurable Antennas}

Reconfigurable antennas are becoming an increasing active topic due to the requirement for antennas to provide additional functionality and flexible properties ([Bernhard 2005]). At present, there are multiple modern wireless and radio frequency (RF) sensing systems intended for different applications (e.g., mobile communications, satellite communications, military applications, radio astronomy, medical imaging...) which require systems with increasing functionality (e.g., direction finding, radar, control, command, beam steering, tracking, etc.). In addition, with the global expansion of telecommunications, operating environments are becoming ever more challenging due to multiple factors, such as the "spectrum crunch", caused by the massive use of communications, or the complex clutter and multipath/propagation backgrounds found e.g., in urban or indoor environments ([Guerci 2014]). Consequently, there is an increasing need for systems that can adapt continuously to an ever changing transmission channel in an "intelligent" and sophisticated way. In this new context, the benefits of reconfigurability are being reappraised and recognized by the industry. Therefore, antenna reconfiguration appears to be a solution and an important direction in antenna design. For example, having different antennas for each of the multitude of wireless standard protocols which exist today (e.g., GSM, UMTS, Bluetooth, WiFi, WiMAX, DSRC, etc.), is no longer a good enough solution. Instead, a single reconfigurable antenna is able to implement the 
functionality of multiple antennas, reducing space and costs. In the field of spatial communications, antenna reconfigurability is clearly very appreciated because it can dramatically reduce cost by allowing antennas to be retasked via programming from the ground rather than requiring completely new satellites to enable new functions and all of the cost that this entails ([Bernhard 2005], [Christodoulou 2012]). Also, reconfigurable antennas have been proposed to be good candidates for the implementation of novel systems based on Software Defined Radio (SDR), because of their capability to adapt and control via microcontrollers ([Perruisseau-Carrier 2010-I]). Likewise, current Multiple-Input-Multiple-Output (MIMO) systems can benefit from reconfigurable antennas by introducing an extra degree of freedom which enhances the system performance ([Christodoulou 2012]). Another important example of utility of reconfigurable antennas is related to the mitigation of the aforementioned "spectrum crunch", that is promoted by the appearance of Cognitive Radio (CR) ([Perruisseau-Carrier 2010-I]); one of the paradigms of major interest at present in the wireless communication field. Cognitive Radio is conceived to provide additional intelligence to communication systems in order to manage dynamically the spectrum efficiently. These systems will require the availability of multi-functional antennas, which integrate multiple reconfigurable characteristics in one single device. Thus, simple and flexible RF front ends can be conceived ([Yashchyshyn 2010-II]), making the implementation of such systems possible and without prohibitive costs.

The field of antenna reconfiguration is currently progressing to the design of simpler and compact solutions which provide higher performance, being able to tune one or different antenna parameters. According to the reconfigurable parameters, reconfigurable antennas are usually classified in four categories ([Costantine 2009]): frequency reconfigurable antenna, polarization reconfigurable antenna, pattern reconfigurable antenna, and multi-function antennas, which is a combination of previous three categories. The corresponding reconfigurability for each category will require of a change in the antenna surface current distribution, a change in the feeding network, a change in the antenna physical structure or a change in the antenna radiation edges ([Christodolou 2012]). Typically, the goal is to alter one or more of these characteristics independently of the others. Therefore, it is essential that engineers take into account that any of these changes can affect to other parameters, so the design procedure must be careful in order to optimize all the antenna characteristics simultaneously and obtain the required reconfigurability.

There are a number of reconfiguration mechanisms to enable tunability. They can be categorized principally in four groups: 
- Mechanical e.g., employing reversible structure modifications, such as rotations, or electromechanical devices, such as microelectromechanical systems (MEMS), piezoelectric actuators or microfluidic.

- $\quad$ Electronic, employing electronic switches, e.g., PIN diodes, or electronic tunable devices, e.g., varactor diodes (varactors) or FET transistors.

- $\quad$ Photosensitive e.g., employing optical switches made of semiconductor materials (e.g., silicon, gallium...)

- Electrical/Magnetic by using special materials (so called smart materials) with controllable permittivity, permeability or conductivity (e.g., ferrite substrates, liquid crystal, semiconductor plasmas, graphene, etc.)

Typically, each antenna is designed to deliver functionality for a specific application. Therefore, according to the type of application to which the antenna is intended, different topologies have been studied for integrating additional functionalities, and find out their potential in this field, since some structures are more suitable for this purpose than others.

Low gain radiators such as microstrip, slot or dipole/monopole antennas have been highly reconfigured for mobile and portable communications or other applications where factors such as wide bandwidth, multiband operation, reduced size, low cost or low observability, can play a key role ([Bernhard 2005]). For example, frequency reconfigurable antennas with discrete change between two or more frequency bands have been achieved by structural modifications controlled by mechanical reconfiguration systems ([Bernhard 2001], [Tawk 2011]), or strategically locating electronic switches, such as PIN diodes ([Gupta 2000]), MEMS ([Anagnostou 2006], [Balcells 2010], [Cetiner 2010], [Topalli 2009],), optical switches ([Freeman 1992]), on the radiating structures. A photograph of a frequency reconfigurable slot antenna with eight PIN diodes in two different configurations that produce operation at two separate frequencies is shown in Fig. 1.1.1(a). Recently, microfluidic switches, based on the flow of a conductive fluid through air-filled microchannels, have been analyzed for a frequency reconfigurable slot antenna able to operate at two different operating frequencies ([King 2013]), as depicted in Fig. 1.1.1(b). These frequency reconfigurable designs offer frequency selective functionality which can reduce the adverse effects of co-site interference and jamming. It is worth to note that an alternative solution to achieve frequency reconfigurability is based on a wideband antenna (e.g., Vivaldi antenna as employed in [Tawk 2012]) combined with a tunable band-pass filter integrated in the radiator feeding line, resulting in a frequency switchable "filtenna". This concept is attractive since it does not require the insertion of switching components directly in the radiating element, although it may have other issues (e.g., related to noise rejection or compactness of the structure) to take into account ([Haider 2013]). Employing tunable reactive loadings (e.g. varactors or FET components), rather than switches, continuous 
tunability can be also achieved ([Korosek 2006], [Hum 2010]). All these structures are based on the reconfiguration of their electrical length, by isolating parts of the radiating aperture (case of switches) or tuning their reactance (case of tunable elements such as varactors), to make the structure resonate at the desired frequency bands. Based on the same principles, frequency reconfigurable Yagi antennas have been achieved, integrating switches or variable reactance devices on the parasitic dipoles, for multiple-band reconfiguration and high-gain ([Cai 2012], [Yang 2004]). Fig. 1.1.1(c) shows a frequency reconfigurable Yagi antenna prototype which attains a $46 \%$ continuous tuning frequency bandwidth with high gain and almost constant endfire pattern ([Cai 2012]). Also, polarization reconfiguration can be integrated by reconfiguring the feeding network in some way ([Balcells 2010], [Li 2010], [White 2009]). Fig. 1.1.1(d) shows a microstrip antenna loaded with MEMS for both frequency and dualpolarization reconfigurability.
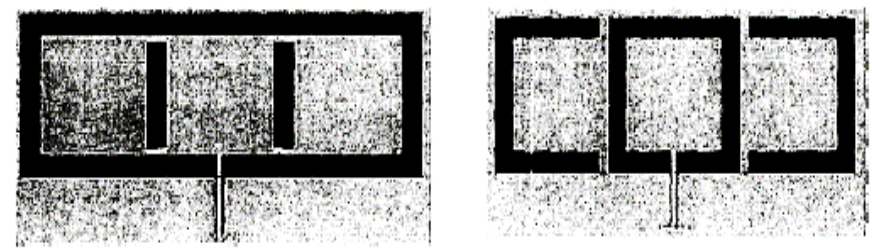

(a)

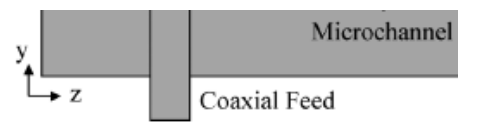

(b)

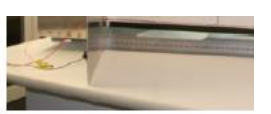

(c)

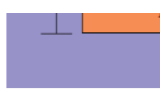

(d)

Fig. 1.1.1 (a) Photograph of a reconfigurable rectangular slot antenna in two different configurations (after [Gupta 2000]). (b) Fluidic frequency reconfigurable slot antenna (after [King 2013]). (c) Yagi antenna with varactor diodes for frequency reconfiguration (after [Cai 2012]). (d) Frequency and polarization agile microstrip patch antenna (after [Balcells 2010]).

Related to Yagi antennas, other small arrays of low gain antennas with electronically tuned or switched parasitic elements close to a driven element can provide interesting pattern variability, while maintaining impedance match and bandwidth. ([Harrington 1978], [Huff 2006], [Jung 2006], [Djerafi 2008]). Fundamentally, tuning of antenna radiation patterns in this manner relies on the change of the mutual coupling between closely spaced driven and parasitic elements, resulting in effective array behavior from a single feed point ([Bernhard 2007]). One of the first and fundamental examples of this type of "parasitic tuning" antenna was proposed by Harrington in 1978 ([Harrington 1978]), shown in Fig. 1.1.2, where the single dipole element in 
the center of the array is driven, while tunable reactance on the surrounding parasitic dipoles couple to the source antenna and produce a high-directive beam in a desired direction.

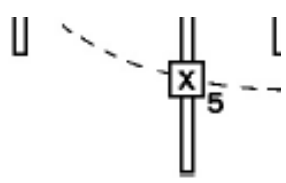

(a)

Fig. 1.1.2 A seven-element circular array of reactively loaded parasitic dipoles for reconfigurable beam steering and beamforming (after [Harrington 1978]).

Unlike previous low gain radiators, high-gain antennas have the ability to efficiently direct the injected power in a desired direction of free space, or equivalently, to receive energy preferentially from a desired direction. For this reason, high-gain reconfigurable antennas are much desired for many applications which require of long-distance communication systems, radar or more expensive and specialized systems, such as satellites, airborne platforms or longhaul land-based communications. Presently, a variety of antenna types are proposed for highgain applications with additional functionalities such as multiband, beam-steering or beamforming. Some examples have been already mentioned, such as Yagi antennas, or parasitic tunable arrays, however there are other notable examples which are currently hot topics in the field of reconfiguration, stimulated for the applications aforementioned, and which are intended to conceive new reconfigurable aperture antennas. Some of them are not new, such as phased arrays or reflector antennas, whereas others are being currently studied in this field since just a few years ago; we are mainly referring to reflectarrays, array lens antennas, pixelled antennas and leaky-wave antennas.

Phased-array antennas have been studied for decades especially for frequency and pattern reconfigurable functionalities. Historically, they were employed for military applications, however, arrays are now being used in a much broader set of scenarios to provide additional functionality and security ([Bernhard 2005]). Phased arrays are well known because of its reconfiguration flexibility, which, unlike aforementioned parasitic tuning antennas, is traditionally delivered through control of the signal magnitude and phase of each one of the identical antenna elements which constitute them, arranged either periodically or aperiodically. This can be achieved by the integration of phase-shifters/true-time delays, circulators, 
attenuators and/or amplifiers in the feeding network, which allow to conform the desired wavefront, as observed in Fig. 1.1.3.

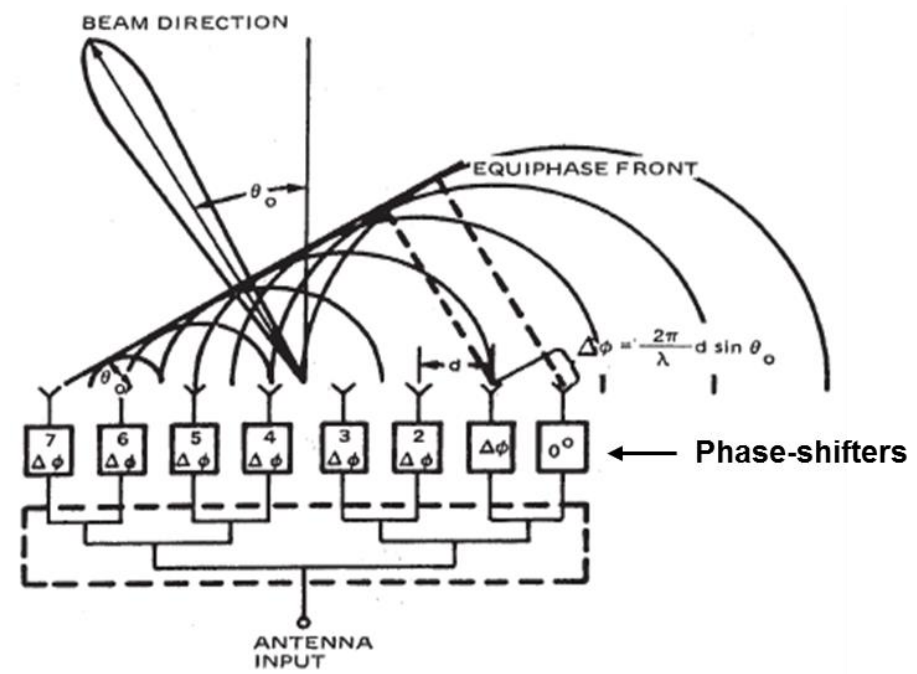

Fig. 1.1.3 Beam-steering phased array using phase-shifters at each radiating element (after

[Hansen 1998]).

For example, one-dimensional (1D) phased arrays can produce fan beams narrow in one plain, and wide in the orthogonal plane, which can be steerable in elevation or azimuth (depending on the orientation of the antenna). Two-dimensional (2D) phased arrays introduce a higher degree of freedom, allowing to produce pencil beams (narrow in both orthogonal planes) steerable simultaneously in both azimuth and elevation. Simultaneous frequency/pattern reconfigurable phased arrays have been also implemented by employing arrays of aforementioned "filtennas", or employing different radiating elements for different frequency bands ([Haider 2013]). However, all these systems suffer of technical issues which complicate the design of these systems as the number of elements or operating frequency increases. Additional losses introduced by phase-shifters are usually compensated by integrating additional amplifiers located immediately near the antenna radiating elements; however, this becomes an important technical issue which raises much the price for some applications where large arrays with hundreds of elements are required. Also, the complexity of the feeding network is cumbersome in this situation. Furthermore, many new current applications are being envisaged to take advantage of higher frequency bands (millimeter waves, THz...). In these cases, the interelement spacing required to avoid grating lobes becomes prohibitively small. For these reasons, new investigations on phased arrays and amplifiers are focused on the miniaturization and enhancement of the efficiency of these devices, for example employing MEMS to reduce cost and loss associated ([Rock 2008]). 
These issues have been some of the stimulus for the industry to look for alternative technologies which offer same or higher performance under a more compact solution. In this context, new reconfigurable aperture antennas have arisen. In concept, reconfigurable apertures represent the ideal functionality of reconfigurable antennas in that they allow the complete specification of radiating currents that can produce any desired frequency and radiation behavior. The challenge lies in developing real structures and control mechanisms that can support this functionality ([Bernhard 2005], [Manasson 2010]).

Reflector antennas are excellent candidates for pattern reconfiguration applications and frequency independent tuning, since the reflective surface is physically removed and isolated from the primary feed. Mechanical positioning or deformations of the reflector (or subreflector) surface result in changes in the currents on the reflector, which in turn alter the aperture field, and finally, the radiation pattern of the antenna. First proposals to reconfigure such type of antennas are based on directly rotating the whole system (reflector antenna + feeding antenna), as observed for example in radio astronomy or space communication, where the antennas are reoriented to point at a fixed target in the sky or to maintain the communication with a satellite, as the Earth rotates. However, these systems are usually bulky, slow and require of periodic maintenance of the mechanism, what for many applications is not feasible. Other electromechanical alternatives arisen to modify directly the main reflector surface. For example, in [Clarricoats 1991], it was demonstrated pattern reconfiguration with a reflector antenna by changing the structure of a mesh reflector by computer-controlled stepper motors. A cheaper solution was proposed in [Washington 2002] deforming a subreflector by piezoelectronic actuators, remaining the shape of the main reflector fixed.

Reflectarrays have appeared as an immediate alternative to conventional reflector antennas and phased arrays for electronic beam-scanning and general beam-forming applications ([Hum 2014], [Perruisseau-Carrier 2010-I]). Reconfigurable reflectarrays combine the simplicity and low-losses of reflector antennas with the planarity and pattern versatility of phased arrays. On the other hand, the bandwidth of reflectarray antennas is significantly narrower than that of reflectors. These antennas are usually composed of a flat textured surface (the main reflector) which is illuminated by an external basic source, such as a horn antenna (as depicted in Fig. 1.1.4(a)). This surface can be comprised of one or various layers of dielectric substrates with periodic tunable scatterers ([Barba 2007]) combined with electronically controllable elements, such as PIN diodes, ([Carrasco 2012-II], [Rodrigo 2013]) varactor diodes ([Boccia 2010], [Hum 2005, 2007], [Rodrigo 2013], [Sazegar 2012], [Sievenpiper 2002, 2003]), MEMS ([Carrasco 2012-I], [Chicherin 2006, 2011-II], [Perruisseau-Carrier 2008, 2010-II]), and tunable materials such as liquid crystal ([Hu 2008], [Perez-Palomino 2012]), ferroelectric substrates ([Romanofsky 2006]) or more recently, graphene ([Carrasco 2013]). Designing properly the 
tunable surface of the antenna with such technologies, the textured surface can be properly reconfigured by a tunable phase gradient, so that these antennas are able to reflect the incident waves producing the desired beam-form and direction of propagation. In this way, significant efforts are devoted to provide additional capabilities to reflectarrays ([Hum 2014]). Reflectarrays have arisen with beam steering at fixed frequency ([Hum 2007]). Also, beamsteering has been achieved simultaneously with flexible polarization and dual-polarization (with independent beam scanning for each polarization ([Carrasco 2012], [Perruisseau-Carrier 2010II])), with dual band ([Guclu 2012]), with amplification capabilities ([Clark 2003]) and, more recently, with multi-band ([Rodrigo 2012-III]).

Array lens (also called transmitarrays), have been also proposed in this field ([Hum 2014]). These structures are excited by an external feeder which illuminates one side of the array, while the radiation is produced on the opposite side. The array is usually composed of a single or multi-layer substrates with different tunable scatterers which allows the electronic control of the transmission properties of the structure (Fig. 1.1.4(b)). This topology has several advantages over their reflectarrays equivalents. First, array lens designs are free from feed blockage effects, which may be a consideration in small apertures. Also, in addition to far-field beam-forming, array lenses have the potential capacity to form focal points in the vicinity of the lens aperture which can be useful in applications requiring adaptive focusing, such as microwave hyperthermia. Reconfigurable transmitarrays have also been implemented in a number of electronic switches, devices, and tuning material ([Boccia 2012], [Lau 2012], [Sazegar 2012]). One of the most outstanding properties recently demonstrated is their potential for electronic beam-forming capabilities employing varactor diodes ([Hum 2014], [Lau 2012]).

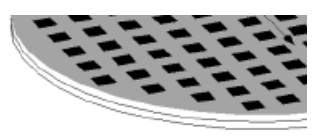

(a)

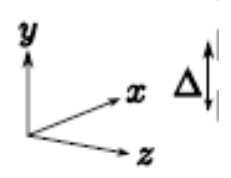

(b)

Fig. 1.1.4 (a) General scheme of a printed reflectarray antenna (after [Huang 2008]). (b) General scheme of a printed transmitarray antenna (after [Sazegar 2012]).

One of the principal drawbacks of any reflector antenna or transmitarrays falls on the necessity of an external protruding source to illuminate the surface, what complicates its integration with 
systems where a reduced volume is required. In this sense, more compact solutions, with a more streamlined geometry that integrate the source embedded in the structure itself are being investigated for these cases.

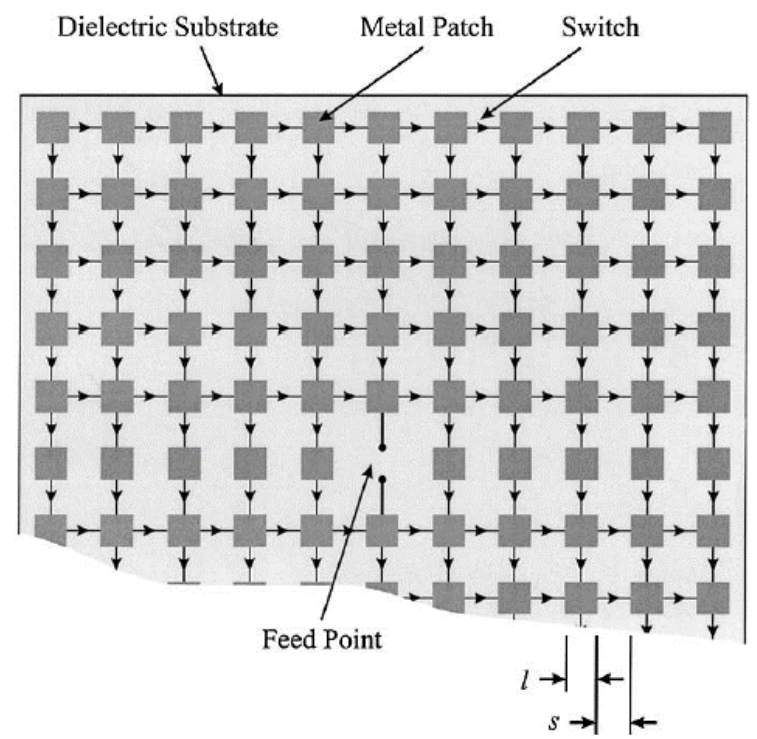

(a)

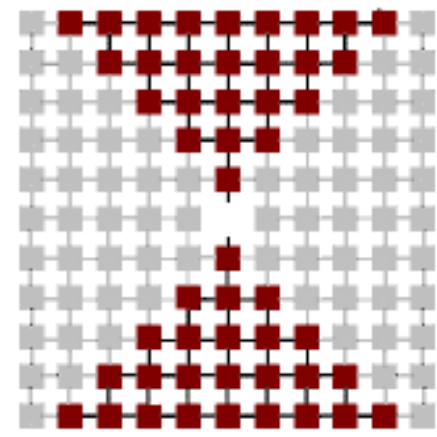

(b)

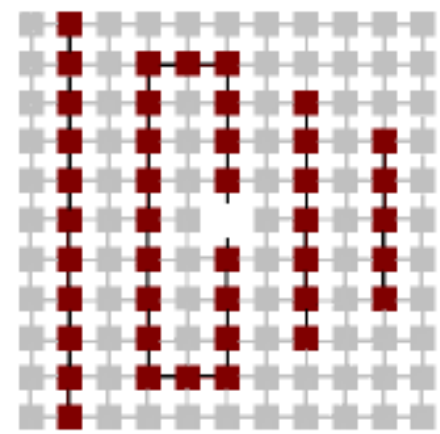

(c)

Fig. 1.1.5 (a) General scheme of a pixelled antenna (after [Pringle 2008]). Configurations of the switches to configure (b) bow-tie antenna and (c) Yagi antenna.

Another type of reconfigurable aperture antenna is a pixelled antenna ([Maloney 2000], [Pringle 2004], [Weedon 2001]). The terms self-structure or evolving-antenna have been also used to define similar concepts. These antennas are based on a grid of electrically small elements (e.g. metallic patches) interconnected by RF switches, which according to their state, allow or not the flow of current, providing reconfigurability of the current distribution over the antenna surface. The scheme of a pixelled antenna with switches for interconnecting non-resonant conductive pads can be observed in Fig. 1.1.5(a). One of the disadvantages of these antennas is that the number of switches can easily number into the thousands and generally, what slows down the optimization algorithms which are usually employed to find out the best configurations to attain 
the required performance goal. However, these antennas are able to obtain from one single reconfigurable array diverse radiation patterns, according to the configuration chosen for the switches, as observed in the examples presented in Fig. 1.1.5(b) and (c). From this principle, several structures can be envisaged. For example, most recent advances in this field have demonstrated how a MEMS reconfigurable pixelled antenna can be used for beam-steering and frequency reconfiguration with multi-size pixels ([Rodrigo 2012]). Also, other basic antennas, such as dipoles or horn antennas, have been combined with tunable pixel layers with different geometries to conceive new devices which incorporate frequency and pattern reconfigurability ([Rodrigo 2011]).

Based on the same principle, but with a less conventional architecture, a new type of reconfigurable aperture antenna based on semiconductor plasma has been proposed. One of the motivation of these structures is to avoid having dozens or hundreds of separated switches on the aperture (avoiding the bias networks that this entailed), although only some switches are involved in the generation of the desired radiation pattern, as occurs with pixelled antennas. The mechanism of these antennas, developed by researchers at the Sarnoff Corporation ([Fathy 2003], [Taylor 2003-I, II]), relies on high conductivity plasma islands that are temporarily created by DC currents that have been injected into high-resistivity silicon-based diode structures. Thus, only the necessary switches are temporarily created in the semiconductor substrate which constitutes the antenna itself. These diodes are also called surface PIN diodes or S-PIN ([Taylor 2003-II]).

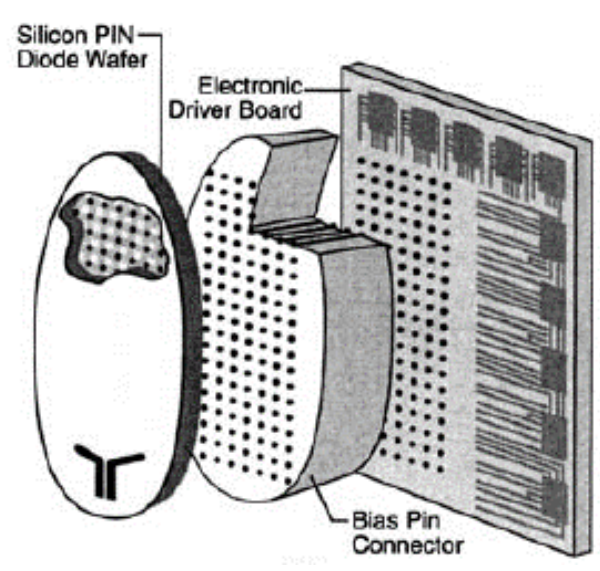

(a)

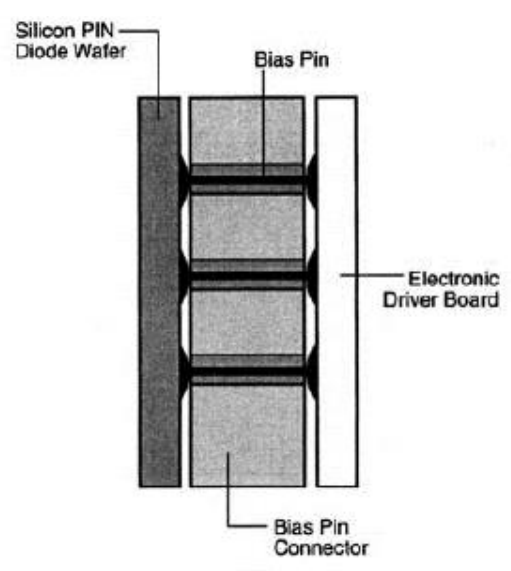

(b)

Fig. 1.1.6 (a) Depiction of a controllable plasma grid structure for a reconfigurable aperture: The grid structure is fabricated on top of the silicon wafer. (b) A cross-section of the plasma grid structure shows the layer interconnection (after [Fathy 2003]).

In the OFF state, when the S-PIN diode is not biased, it presents the high resistance of the area between the doped regions. In the on state, the S-PIN diode then represents a layer of highly 
conductive material. The resistance of an activated S-PIN device depends on the level of the forward current and the carrier lifetime at the plasma region. Fig. 1.1.6 contains a detailed description of the structure, including the plasma injection driver configuration. Thereby, the regions dominated by each S-PIN diode define the antenna structure, and they can be changed to create different antennas. While elegant in its conception, the embodiment is fundamentally as complex as the previous pixelled antennas. This means that many practical and technical issues exist with this approach, including the challenge of creating the necessary carrier densities in the silicon. Nevertheless, the applicability of these structures is promising for future applications in microwave and mm-wave applications ([Yashchyshyn 2010-I, II]). At present, holographic antennas based on a semiconductor chip that contains a set of individually controlled S-PIN diodes have been proposed. Electromagnetic waves propagate through the chip, which also serves as a planar dielectric waveguide excited by e.g., a mounted dipole antenna in the substrate. Through selective activation of S-PIN, metallization patterns can be simulated, which are excited by the propagated surface wave ([Taylor 2003-I]). The aperture field for the desired pattern is then produced through interaction with the plasma aperture, enabling beam-steering and beamforming without the need for expensive phase shifters. Likewise, this approach can be also appropriate for creating intermediate apertures illuminated by more basic sources, as in the case of reflectarrays or previous pixelled antennas. In this case, the impinging wave interacts with the plasma aperture, which is reconfigured to create the desired reflected wavefront ([Bernhard 2005]). The plasma aperture could be designed using iterative methods or other means and reconfigured to deliver performance comparable to traditional phased arrays. Recently, arrays of S-PIN diodes have been combined with the radiators of a waveguide slot antenna in order to achieve electronic beam-steering and frequency tuning capabilities ([Yashchyshyn 2010-I]). These antennas are a potential alternative to e.g., PIN biasing circuit complexity, but their nonlinearities, as well as antenna efficiency, would still require further investigations.

One of the antenna types which is attracting more attention since the last decade in the field of high-gain electronically reconfigurable aperture antennas is the leaky-wave antenna (LWA). LWAs have been in the antenna scene for many decades because of their simplicity and highgain characteristics ([Oliner 2007]). Today, several books on this topic can be found. ([Caloz 2010], [Hessel 1969], [Jackson 2008], [Oliner 2007], [Tamir 1969], [Walter 1965]). These antennas can be regarded as transmission lines that gradually radiates ("leaks") into free space the energy of the waves which propagate through the structure (hence its name, leaky wave). LWAs are able to produce narrow beams which direction, $\theta_{R A D}$, is determined by the phase constant $(\beta)$ of the leaky wave along the transmission line, while the beamwidth is determined by the length of the aperture, and the leakage rate $\alpha$ ([Oliner 2007]). However, due to the 
natural dispersion of leaky lines, the bandwidth of LWAs is usually narrow. Nevertheless, the principal advantages which have generated interest in this type of antennas are their simple structure, often planar or low profile (with only a modest depth requirement) and integrated excitation, what offers more compactness, easy integration with other microwave circuits and design simplicity if compared with large phased arrays or pixelled antennas, which require of complex feeding networks, or with reflector antennas, which need of protruding external feeds to illuminate the structure. Regarding the structure, a wide variety of different one-dimensional (1D) and two-dimensional (2D) geometries have been built to support such waves. Initially, LWAs were built by open waveguides ([Hansen 1940], [Hines 1957], [Rotman 1959]) or dielectric rods ([Peng 1978]). Later, microstrip lines excited with the higher order mode were studied ([Menzel 1979]). ([Oliner 2007]). Since then, a number of technologies have been employed to conceive low cost LWAs implemented in planar technologies such as e.g. microstrip, coplanar waveguide, slot line, substrate integrated waveguide, etc. ([Grbic 2002], [Deslandes 2005], [Martínez-Ros 2012-I, 2013-I], [Menzel 1979]...), or low-profile hybrid technologies ([Lampariello 1987], [Gómez-Tornero 2005-I], [Garcías-Vigueras 2012-II]...). In addition, it is worth to note that many of these technologies allow scalability of LWAs, what makes them also attractive not just for microwave applications ([Oliner 2007]), but for millimeter-waves ([Grbic 2002], [Manasson 1995], [Chicherin 2006, 2011-I, II], [Oliner 1988, 1990], [Raisanen 2012]) and even THz ([Jackson 2011] [Esquius-Morote 2014]). However, the lack of necessary fixed frequency reconfigurable elements is still the obstacle which is preventing the realization of tuneable LWAs at these higher frequency bands.

In this dissertation, we focus on the electronic reconfiguration of this type of antennas. Although detailed state-of-the-art revision and background knowledge is provided throughout the next chapters, below, an overview on some of the most significant reconfigurable LWAs is now given in order to clarify the context that has motivated this $\mathrm{Ph} . \mathrm{D}$. One typical property of LWAs which has been widely exploited for reconfiguration purposes is frequency scanning ([Oliner 2007]). This characteristic, which is inherent to these structures because of the aforementioned dispersive nature, confers to LWAs the capability to steer its main beam just sweeping the operating frequency, what has been interesting for some scanning applications, but finds limited applicability in current modern systems, such as point-to-point communications, where it is usually required to operate at a fixed frequency. Advances in dispersion engineering of these transmission lines have risen to novel leaky-wave guiding structures with natural properties which allow higher directivity, backward-to-forward frequency scanning, wide bandwidth, dual-band scanning (scanning at two discrete operational bands), dual-polarization, circular-polarization, etc. ([Bruni 2007], [Debogovic 2014-I], [Duran-Sindreu 2013], [Feresidis 2005], [Kelly 2007], [Kodera 2009], [Krauss 2011], [Jackson 1988], [Jackson 1993], [Ju 2012], 
[Machac 2013], [Moghadas 2013], [Neto 2003], [Orr 2014])...). Some of the most notable leaky-wave based theoretical developments, structures and systems appeared during the last decade can be found in recent books such as [Jackson 2008] or [Caloz 2010]. Regarding electronic reconfigurability, one of the potentials of many leaky-wave structures is its simplicity to be combined with tunable devices, such as tunable elements, electronic switches or tuning materials. Thus, the dispersion characteristics of the leaky lines can be altered, controlling the wave propagation velocity, and hence, reconfiguring the aperture illumination of the antenna. In this way, several types of LWAs have been conceived, especially for multiband and fixedfrequency beam-steering functionalities. Some notable examples are presented below. For example, different topologies of dielectric LWAs have been loaded with PIN diodes, MEMS, photosensitive switches or electrical switches for beam-switching among different discrete angles ([Alphones 1995], [Horn 1980], [Huan 2000], [Chang 1999], [Chicherin 2011-I, II], [Karmokar 2013], [Li 2006, 2008]). In these cases, the number of possible angles is given by the density of switches along the line and the number of combinations ([Karmokar 2013], [Manasson 2010]). Luxey and Noujeim studied the effect of reactive loads on microstrip LWAs, observing potential for continuous beam-steering at fixed frequency ([Luxey 2000], [Noujeim 1998, 2003]). Continuous scanning is now commonly achieved employing tuning devices such as varactors ([Sievenpiper 2005]) or MEMS varactors for higher frequency applications ([Chicherin 2011-I], [Zvolensky 2010]). One of the most typical examples of continuous beamsteering LWA is depicted in Fig. 1.1.7, where it is shown a textured surface loaded with varactor diodes which was presented in [Sievenpiper 2005]. This surface is constructed as a printed circuit board, with metal plates on the front side connected to a ground plane on the back by vias. Varactor diodes are connected between each pair of plates (Fig. 1.1.7(a)). Half of the plates are connected to a ground plane on the back by metal plated vias, and the other half are connected to bias lines that control the varactors by independently biased rows (Fig. 1.1.7(b)). A horizontally polarized flared notch antenna excite TE surface waves in the dielectric slab, which are transformed into forward (Fig. 1.1.7(c)) and backward (Fig. 1.1.7(d)) leaky waves when interact with the tunable textured surface, achieving fixed frequency continuous beam-steering at first and second quadrant. Notice that backward radiation is achieved by applying two alternate voltages to adjacent rows, which doubles the effective lattice period, exciting a higher order space harmonic which radiates at backward angles ([Oliner 2007]). 


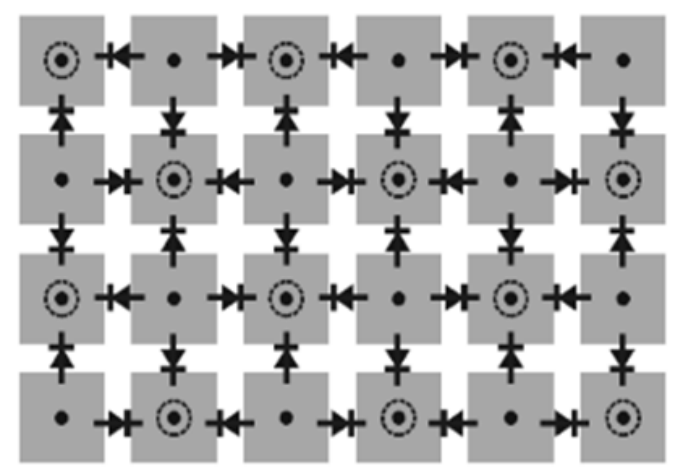

(a)

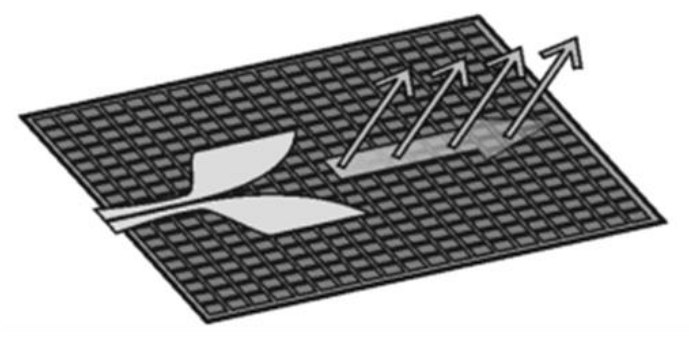

(c)

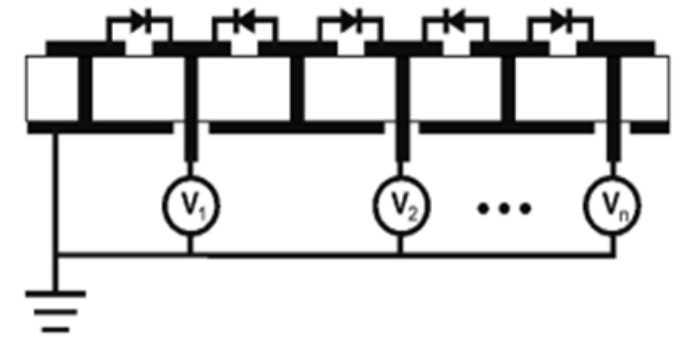

(b)

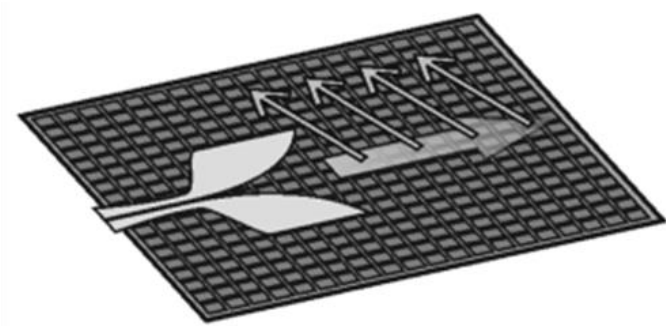

(d)

Fig. 1.1.7 (a) Top view and (b) Side view of a tunable textured surface. This feed can launch (c) forward leaky waves or (d) backward leaky waves (after [Sievenpiper 2005]).

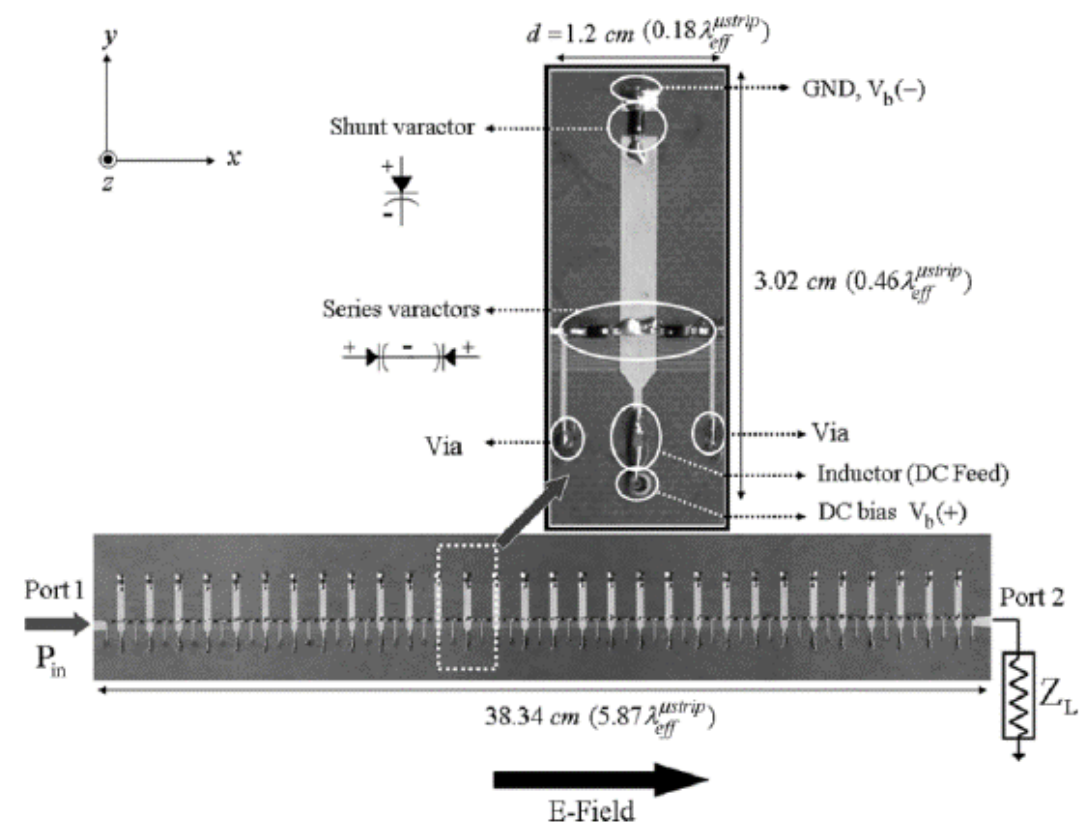

Fig. 1.1.8 30-cell prototype of the voltage-controlled reconfigurable CRLH LWA structure with a magnified view of the unit cell. The E-field polarization is along the $x$-direction (after [Lim 2004-II]).

Another important example of continuous beam-steering LWA, was given by the researchers Lim, Caloz and Itoh, which proposed in [Lim 2004-I] a reconfigurable LWA based on the 
properties of a Composite Right/Left Handed (CRLH) (also commonly referred as metamaterial) transmission line. This antenna is able to scan continuously its main beam from backward to forward, employing different shunt and series varactors in the unit cell which composed the periodic geometry of the antenna. In addition, controlling locally the phase constant of the leaky wave (independently biasing each unit cell), they achieved simultaneously control of the pointing angle and beamwidth ([Lim 2004-II]). Fig. 1.1.8 shows the fabricated prototype, and also shows in detail one single unit cell. After these works, more reconfigurable CRLH LWAs emerged with different capabilities apart from beam-steering ([Matsuzawa 2005, 2006, 2007], [Lee 2007], [Nguyen 2007, 2011], [Ouedraogo 2011], [Piazza 2010] [Suntives 2012]). At present, metamaterials is a hot topic in the field of reconfiguration ([Palmer 2011]).

Also, other LWAs have been built employing tuning materials to build the guiding structure, allowing continuous scanning by changing the permittivity/permeability/conductance of these materials. One of the first trials was with magnetically-biased ferrite substrates ([Maheri 1988], [Varadan 1994]). Now, ferroelectric substrates ([Yashchyshyn 2005-I]), or photosensitive silicon substrates ([Zuliani 2004]) have been conceived, employing electric or optical signals respectively, to control $\theta_{R A D}$. Aforementioned plasma semiconductor substrates have also been employed to excite leaky waves by reconfiguring S-PIN to create the desired pattern ([Taylor 2003-I, II]). Last advances have employed liquid crystal for mm-wave applications ([Matsuzawa 2005, 2006], [Roig 2013]) and even a graphene LWA has been recently proposed for continuous beam-steering at THz bands ([Esquius-Morote 2014]).

A type of LWA which is gaining much attention because of their simple structure, high gain characteristics and potential to integrate reconfiguration, is the Fabry-Pérot (FP) LWA. FP LWAs were firstly introduced by Von Trentini ([Trentini 1956]) in the 1950s, and are basically constituted by a FP resonant cavity composed of two reflective surfaces (for example, a partially reflective surface (PRS) made by a one-dimensional or two-dimensional frequency selective surface (FSS) and a ground plane or a high impedance surface (HIS), as will see) and an embedded (usually punctual) low gain radiator which feeds the whole structure (e.g. a patch antenna, a slot on a ground plane). The control of the aperture illumination is usually based on the reflection properties of these surfaces, which comprise the FP cavity, so tunable surfaces are employed to allow reconfiguration. In most cases, 2D FP LWAs have been employed to produce a pencil beam pointing at broadside with high directivity. Also, these antennas can generate conical beams due to the symmetry of $2 \mathrm{D}$ structures, however, although there might be potential applications for FP LWAs having conical beams ([Costa 2011]), in general it is desirable to preserve the maximum radiation in the normal direction for all states of the antenna ([Debogovic 2010]). For example, a FP LWA was proposed in [Weily 2008] for multi band operation, keeping constant a high gain pencil beam pointing at broadside at different frequency 
bands. To this aim, the FP cavity, which was centrally excited by a stacked patch antenna, was loaded with a tunable HIS with varactor diodes at the bottom of the cavity, as can be observed in Fig. 1.1.9. This tunable surface was in charge of tuning the FP resonance frequency, allowing operate from $5.2 \mathrm{GHz}$ to $5.95 \mathrm{GHz}$ (13.5\% tuning range). Similar applicability has been pursued in other works ([Ourir 2007], [Burokur 2013]).

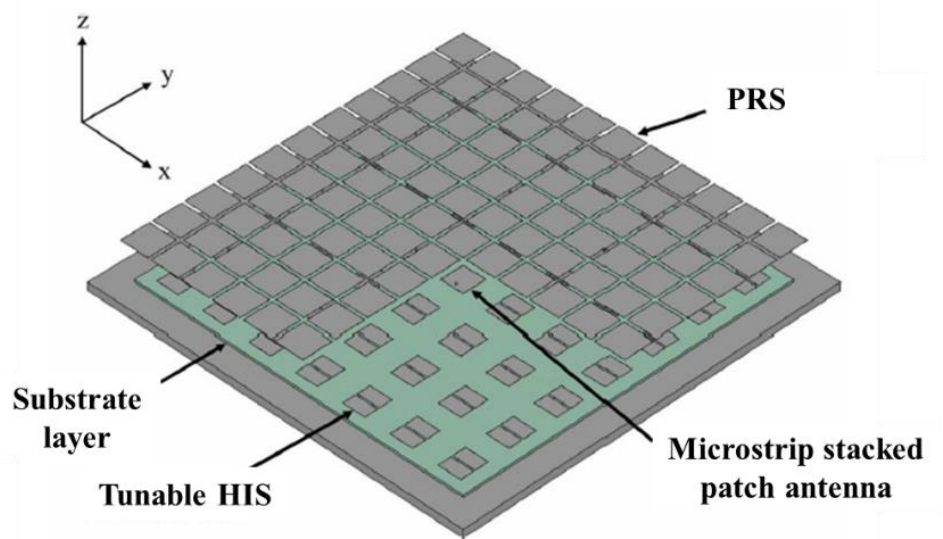

Fig. 1.1.9 Cutaway drawing of the frequency reconfigurable FP LWA. One quarter of the PRS material has been removed to show the details of the antenna more clearly (after [Weily 2008]).

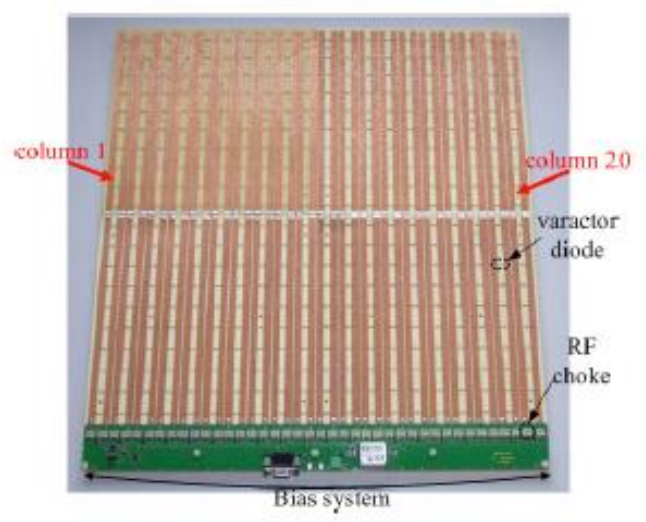

(a)

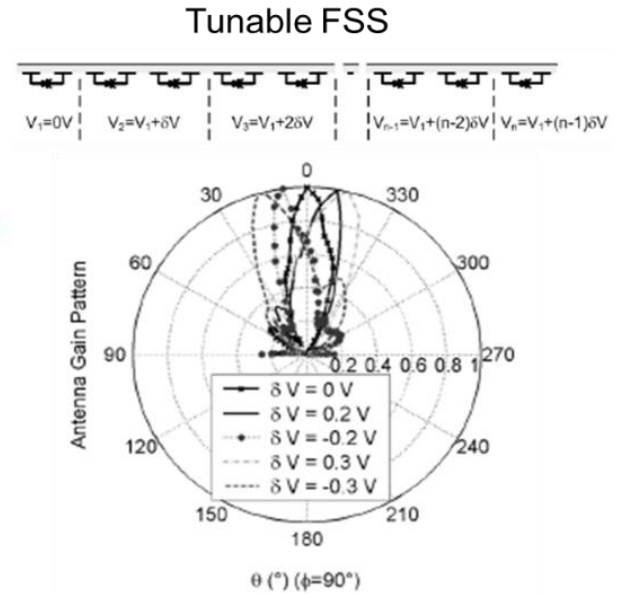

(b)

Fig. 1.1.10 (a) Electronically tunable metasurface reflector. (b) Measured gain patterns in the Eplane $\left(\phi=90^{\circ}\right)$ at $7.9 \mathrm{GHz}$ for $\delta \mathrm{V}=0 \mathrm{~V}, \delta \mathrm{V}=0.2 \mathrm{~V}$ and $\delta \mathrm{V}=0.3 \mathrm{~V}$. The steering of the antenna's radiated beam can be clearly observed with a positive steering angle for positive bias and negative one for a negative bias (after [Burokur 2013]).

Other works have proposed tunable PRS to control the beamwidth of the pencil beam for zoom applications ([Edalati 2007], [Wang 2014], [Debogovic 2010, 2014-I, II]). These works are based on the control of the leakage rate of the leaky wave through a tunable PRS which resonance response (transparency) can be electronically tuned by PIN diodes ([Edalati 2007]), varactor diodes ([Debogovic 2010, 2014-II]) or MEMS ([Devogobic 2014-I]). Also, fixed- 
frequency beam-steering capability has been attempted with these structures to scan a conical beam employing a tunable HIS with varactors ([Costa 2011]), or a ferroelectric superstrate ([Lovat 2007]). Electronic steering of a pencil beam have been recently developed with FP structures ([Ourir 2009], [Burokur 2013], [Debogovik 2014-II]), avoiding the natural evolution of FP LWAs into a conical beam. A two-dimensional FP LWA constituted by a ground plane and a tunable metasurface with varactors has demonstrated this capability ([Ourir 2009], [Burokur 2013]). The metasurface reflector employed as PRS is depicted in Fig. 1.1.10(a). This PRS imposes a phase-gradient along the FP cavity, as a function of the bias voltage applied to the row of varactors, allowing scanning of the pencil beam in the E-plane. The radiation patterns obtained for different configurations are depicted in Fig. 1.1.10(b). Also, in [Debogovik 2014II] a similar FP LWA, depicted in Fig. 1.1.11, was conceived to scan its pencil beam in the Hplane, but not through a tunable PRS, but through the phase shift of a small phased array of patch antennas embedded into the FP cavity to excite the structure. In addition, this antenna implemented the possibility of changing the beamwidth of the pencil beam by a reconfigurable PRS with varactors.

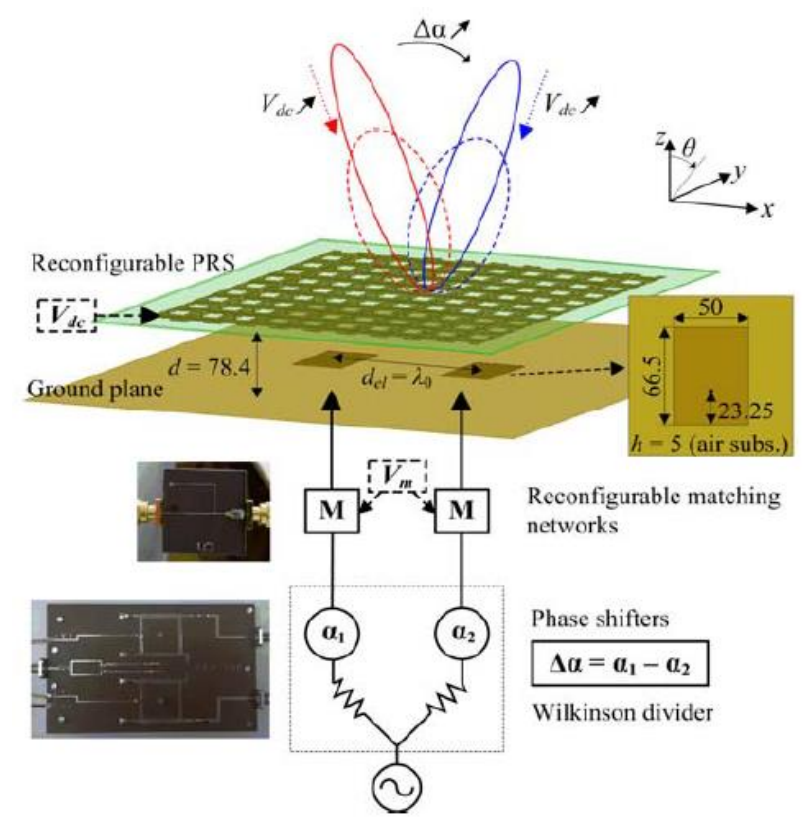

Fig. 1.1.11 Proposed array-fed PRS antenna with independent dynamic beamwidth control and beam-scanning in the H-plane ( $X Z$-plane). All physical dimensions are in millimeters (after [Debogovic 2014-II]).

In some examples presented above, it is usually employed complex structures which require of a high number of control signals or complicated design procedures to obtain the desired reconfigurable properties. In this context, and following the current tendency of reconfigurable LWAs, we proposed in this research work novel reconfigurable LWAs which take advantage of 
the concepts and properties of FP LWAs (simplicity and high gain), to obtain pattern reconfiguration capabilities in a simple way. To this aim, this work presents a simple but accurate methodology to characterize and design these structures, allowing to analyze in a fast and efficient way the response of the antenna, prior to perform full-wave methods. For first time, pattern reconfigurable 1D and 2D FP LWAs are conceived just employing their dispersion properties, without necessity of dielectric substrates with gratings, external switches or metamaterial lines, to obtain half-space and full-space beam steering capabilities. Final studies have demonstrated experimentally how a 2D FP LWA with a tunable HIS can be employed for two-dimensional beam steering of its pencil beam, in azimuth and elevation, so that the antenna can form a beam in practically any direction within a wide scanning range. In addition, it is believed that the reconfiguration mechanisms studied in this thesis can be scaled to conceive reconfigurable antennas at higher frequencies. 


\subsection{Motivation and Objectives}

Despite the increasing investigations performed during the last decades in the field of reconfigurable antennas ([Bernhard 2005]), there is still a need for reducing costs, losses and design complexity to innovate with high-performance multifunctional antennas, what is still a challenge to satisfy all the practical demands of the industry. The design of reconfigurable antennas is usually more complex than for passive (non-reconfigurable) antennas. This complexity is usually associated to the addition of mechanical, electrical or electronic mechanisms to confer their dynamic response. Mismatching issues due to the change of the input impedance with the operating mode, or the difficulties derived from the integration of tunable elements (losses, bias networks, additional control signals...) are also factors to take into account. All these factors notably increase the economic cost of these antennas. Today, the availability of different types of reliable switches and tunable elements has made reconfigurability considerably more practical for antennas than it was even a few years ago, extending the range of their applicability ([Christodoulou 2012], [Haider 2013], [Rock 2008], [Rutschlin 2013], [Yashchyshyn 2010-II]). However, the tendency is oriented to look for technologies which allow for simpler structure, frequency scalable and low cost reconfiguration mechanisms. The principal motivation of this thesis is the proposition of novel reconfigurable antennas with electronic control of the radiation pattern (pattern reconfigurable antennas) employing a simple structure in low cost technology. In particular, the thesis focuses on reconfiguration of Fabry-Pérot (FP) leaky-wave antennas (LWAs). One of the major attractions of these antennas is their structure simplicity to conceive compact high-gain antennas. The author proposes for first time three novel pattern reconfigurable FP LWAs with electronic beam-steering capabilities. These antennas can be considered low profile (with a modest depth requirement) and employ new reconfiguring mechanisms based on the electronic control of the dispersion properties of the cavity to control the beam direction while operating at fixed frequency. Experimental prototypes have been designed, manufactured and tested to operate in the microwave $\mathrm{C}$-band (around $5.5 \mathrm{GHz}$ ) as proof of concept.

The major contributions of the thesis to the field of reconfigurable antennas can be summarized in the following points:

- Proposition of new pattern reconfigurable antennas based on FP LWAs implemented in simple (easily manufacturable) and low profile technology.

- Demonstration of new electronic-reconfiguration mechanisms employing dispersion properties of FP structures.

- Guidelines for the efficient analysis and design of reconfigurable FP LWAs. 
- Discussion on limiting factors and challenges for the design of new low-cost and highperformance reconfigurable antennas.

Below, seven specific technical objectives are described, which together represent the overall goal pursued with the development of this thesis:

O1. Design of a reconfigurable 1D FP LWA with half-space electronic-steering employing a tuneable HIS.

O2. Characterization of FSSs loaded with tunable elements by an extended pole-zero expansion method.

O3. Efficient modal dispersion analysis of FP LWAs loaded with tunable FSS with lumped elements.

O4. Analysis of the pattern bandwidth of reconfigurable beam-steering FP LWAs.

O5. Design of a reconfigurable 1D FP LWA with full-space electronic-steering employing a tuneable HIS and the EBG electronic-routing mechanism.

O6. Demonstration of an electronic-routing mechanism based on the electromagnetic band-gap of periodic FP LWAs.

O7. Design of a 2D FP LWA with simultaneous and independent electronic-steering in azimuth and elevation employing a tuneable HIS and the EBG electronic-routing mechanism. 


\subsection{Thesis Outline and Original Contributions}

In this section, the organization of the thesis is given in order to sum up the principal aspects and contributions demonstrated in each chapter. The principal contents have been divided in four chapters which cover chronologically each step followed during the development of the investigation. Next, a brief description of the work described in each chapter is given.

In Chapter 2, the analysis and design of an original half-space electronic-steering 1D FP LWA is performed. This 1D antenna demonstrate its capability to electronically scan continuously a fan beam in the directive plane at the first quadrant (positive angles), just employing a tunable High Impedance Surface (HIS) loaded with varactor diodes. After an introduction to the state of the art in electronic beam-steering LWAs, the bases on LWAs and FP LWAs are reviewed to define the concepts which allow us to deal with this type of antennas. Later, the passive (nonreconfigurable) double-layer 1D FP LWA, recently proposed in [García-Vigueras 2012-II], is analyzed to explain the control mechanisms which have inspired the starting hypothesis of the reconfiguration mechanism of our electronically-steerable antenna. Then, we move on the structure of the proposed reconfigurable 1D FP LWA. An efficient modal dispersion analysis is performed by a TEN of the structure, and a characterization method is developed to integrate the effect of the reflective surfaces which comprise the FP cavity. Experimental results obtained from a fabricated prototype are shown to demonstrate the proof of concept. Also an efficiency analysis gives interesting information about the effect of losses due to varactors and materials. Finally, a study of the pattern bandwidth has been performed to analyze the effect of beamsquint in fixed-frequency beam-steering reconfigurable antennas. The fulfillment of objectives $\mathrm{O} 1, \mathrm{O} 2, \mathrm{O} 3$ and $\mathrm{O} 4$ are here pursued.

In Chapter 3, it is presented the analysis and design of a novel full-space electronic-steering 1D FP LWA. The structure of this antenna is derived from the previous half-space antenna, which in this case is excited from its center and divided in two independently controllable sections to achieve continuous scanning of the beam direction from positive (first quadrant) to negative angles (second quadrant), besides broadside radiation. To do so, leaky waves launched from the central source are rerouted by a novel concept baptized as electromagnetic band-gap (EBG) electronic-routing mechanism which is based on the electronic tunability of the EBG properties arisen in periodic FP structures. Thus, this mechanism, along with the electronic beam-steering properties demonstrated in Chapter 2, grant the continuous full-space steering capability to the antenna. Simulations and a fabricated prototype demonstrate the scanning properties of the antenna and its maximum scanning range. The objectives aimed in this chapter are O5 and O6. 
In Chapter 4, a two-dimensional version of the previous full-space electronic steering 1D antenna is considered to conceive for first time a 2D FP LWA with simultaneous scanning in elevation and azimuth. The structure is excited from its center and is loaded with a tunable HIS divided in four sectors tuned independently. This antenna is illuminated by cylindrical leaky waves which would generate in a normal homogeneous/symmetrical FP LWA a high-directive pencil beam at broadside or a conical beam when the pencil beam is scanned off broadside. However, thanks to the electronic-steering properties and the EBG electronic-routing concept presented in previous chapters, it has been demonstrated how a 2D FP LWA with a single central excitation (a stacked patch antenna in this case) is able to scan in azimuth and elevation its pencil beam as a function of the four control signals which bias the varactors of each one of the tuning sectors. Thus, a discrete azimuth scanning at eight different angles is obtained, whereas continuous scanning in elevation is achieved. A prototype has been fabricated and measured demonstrating the proof of concept. Objective $\mathrm{O} 7$ is here accomplished.

Finally, in Chapter 5 the most relevant results are compiled. Also, some comments on limiting factors of the presented antennas and short-term research perspectives are provided. 


\section{Chapter 2 Half-Space Electronic-Steering 1D FP LWA}

In this chapter it is performed the analysis and design of a novel fixed-frequency electronic scanning one-dimensional (1D) Fabry-Pérot Leaky Wave Antenna (FP LWA). This antenna is able to electronically control its fan beam direction in the first (positive) quadrant. The structure is implemented in hybrid technology, which results from the combination of a metallic waveguide and two periodic surfaces: a Partially Reflective Surface (PRS), and a tunable High Impedance Surface (HIS) loaded with varactors. The PRS confers the leaky-wave nature to this structure, whereas the tunable HIS will be responsible for the electronic control of the pointing angle. An efficient modal dispersion analysis based on a Transverse Equivalent Network (TEN) has been employed to analyze and design the antenna performance in a fast and accurate way. The analysis and characterization of the frequency selective surfaces (FSS) which compose the PRS and HIS is essential for the dispersion studies and the final design of the antenna. To this purpose, some techniques proposed in recent works based on the pole-zero expansion method have been employed and also extended for the analysis of tunable frequency selective surfaces (FSS) loaded with lumped elements, such as the varactors employed in the tunable HIS. An efficiency analysis has been carried out to analyze the radiation efficiency and lossy effects of varactors and dielectric substrates as a function of the operating point (scanning angle) of the reconfigurable antenna. Also, in order to analyze the pattern bandwidth of the antenna, a simple theoretical formulation has been derived, applicable to any reconfigurable beam-steering LWA, to estimate the maximum instantaneous bandwidth of the proposed antenna. A prototype of the 
electronic-steering 1D FP LWA has been fabricated to operate at $5.6 \mathrm{GHz}$, obtaining very good results between measurements, simulations and theory and demonstrating an electronicallycontrolled scanning region from $\theta_{R A D}=9.2^{\circ}$ to $\theta_{R A D}=34.2^{\circ}$ with a maximum bandwidth of $2.22 \%$. From the general objectives enlisted previously, the following ones will be aimed in this chapter:

O1. Design of a reconfigurable 1D FP LWA with half-space electronic-steering employing a tunable HIS.

O2. Characterization of FSSs loaded with tunable elements by the extended pole-zero expansion method.

O3. Efficient modal dispersion analysis of FP LWAs loaded with tunable FSS with lumped elements.

O4. Analysis of the pattern bandwidth of reconfigurable beam-steering FP LWAs.

The chapter is organized as follows:

In Section 2.1, an introduction to the state of the art on electronically-steerable LWAs is presented. In order to contextualize and deal with the design of the reconfigurable LWAs presented in this thesis, Section 2.2 is aimed to introduce basic concepts about LWAs and an overview on their classification. Section 2.3 focuses on the passive (non-reconfigurable) doublelayer 1D FP LWA presented in [García-Vigueras 2012-II]. After a general introduction to Fabry-Pérot LWAs, the essential radiation principles and main results derived from the analysis of the passive 1D FP LWA are reviewed. These results will be essential for the proper comprehension of the reconfigurable structures presented in this thesis. Section 2.4 shows the reconfigurable structure proposed in this chapter: an electronic-steering 1D FP LWA constituted by a PRS and a tunable HIS with varactors. Its geometry and initial hypothesis about its working principle are described in this section. Also, it is summarized the general methodology followed for the analysis and design of the antennas presented in this thesis. Later, Section 2.5 focuses on the characterization of the FP LWA by a TEN, in order to analyze the structure, and the characterization of the FSS by equivalent admittances applying a pole-zero method. Section 2.6 demonstrates theoretically and by simulation the effect of the tunable HIS for the electronic control of the pointing angle, and the effect of the height of the FP cavity to optimize the dynamic scanning range. A fabricated prototype and experimental results are shown in Section 2.7, confirming the initial hypothesis and validating the theoretical results. Finally, Section 2.8 and 2.9 are dedicated to the analysis of the efficiency and pattern bandwidth of the antenna respectively. Main conclusions of the chapter are summarized in Section 2.10. 


\subsection{Introduction}

Beam-steering is a property of some directional reconfigurable antennas which allows to control the direction of its main beam. This functionality is highly desired for different communication and RF sensing systems increasingly common in the actual panorama of the telecommunications (interference avoidance, adaptability, indoor communications, radar, surveillance, radio astronomy, medical imaging, tracking, localization, communication maintenance, etc. ([Bernhard 2005])). As aforementioned in Chapter 1, leaky-wave antennas (LWAs) have great potential to integrate this property.

Frequency scanning has been one of the very first attractions of LWAs. This property allows to control the beam direction as the antenna operating frequency is swept. Frequency scanning is inherent to the dispersive nature of LWAs and has been widely studied for several leaky-wave structures ([Fralich 1992], [Hu 2000], [Oliner 1979, 1985], [Walter 1965]...) and exploited for some scanning applications ([Bernhard 2005], [Oliner 2007]). However, many wireless communications and modern telecommunication systems normally require operate at fixed frequency at a given frequency band defined by the spectrum regulators (e.g. for establishing fixed point-to-point communication links, or maintaining the communication with a moving target). In these cases, the inherent frequency-scanning property of LWAs has very limited applicability, being necessary other mechanisms which allow to steer the beam operating at the fixed frequency of interest. In this context, electronic beam-scanning LWAs have arisen to achieve steering of the main beam with the structure simplicity and low cost features of these antennas. The aforementioned high dispersive nature which grants frequency scanning to LWAs, is precisely the property which also confers their potential for fixed-frequency electronic-steering reconfigurability. Fixed-frequency electronic steering of a LWA has been usually achieved by introducing in some way electronically-tunable elements, such as electronic switches (e.g., pin diodes, RF MEMS), tunable devices (e.g., varactors or FET transistors) or tunable materials (ferroelectric, plasma, liquid crystal, graphene...), in the leaky structure, what allows to alter in a controlled way the dispersion characteristics of the leaky guide ([Suntives 2012]), and hence vary the leaky-mode complex propagation constant at the fixed frequency of interest. As the leaky-mode phase constant is related with the pointing angle of the antenna, a change in its pointing direction $\theta_{R}$ ([Oliner 2007]) is resulted. Following this hypothesis, several proposals and technologies have been presented in recent decades to create fixed-frequency electronic scanning LWAs. For instance, PIN diodes have been added to dielectric rod onedimensional (1D) LWAs ([Horn 1980], [Huan 2000]) or microstrip LWAs ([Chang 1999], [Karmokar 2013]), obtaining a discrete change in $\theta_{R}$. Similar discrete changes are reported when introducing photosensitive switches in dielectric slab LWAs ([Alphones 1995]), or electronic 
switches in multi-port microstrip LWAs ([Li 2006, 2008]). Continuous scan of the main beam has been obtained employing tunable materials, such as ferrite LWAs ([Maheri 1988], [Varadan 1994]), microstrip LWAs over a ferroelectric substrate ([Yashchyshyn 2005-I], [Lovat 2007]), by respectively using magnetic/electric fields. Also photoinduced plasma grating LWAs ([Manasson 1995]) or microstrip LWAs loaded with photosensitive silicon substrates ([Zuliani 2004]) has been conceived employing optical signals to control $\theta_{R A D}$. Other tunable materials, such as plasma ([Kallel 2013]), liquid crystal, usually employed for mm-wave applications ([Matsuzawa 2005, 2006], [Roig 2013]), or semiconductor substrates with surface PIN diodes (S-PIN) ([Fathy 2003], [Manasson 2010], [Yashchyshyn 2009, 2010]) have been also employed ([Taylor 2003-I], [Yashchyshyn 2005-II]). Recently, a graphene-composed LWA for beamsteering at $\mathrm{THz}$ frequencies has been proposed by virtue of the tunability of graphene's conductivity ([Esquius-Morote 2014]). Also, microelectromechanical systems (MEMS) have been proposed for continuous beam-scanning LWAs ([Zvolensky 2010], [Chicherin 2011-I], [Matsuzawa 2007]). However, the most used active device to electronically control 1D LWAs in the microwave range is the varactor diode, which has been applied to multitude of leaky lines as the slotline ([Chen 2002]), the first higher-order mode microstrip line ([Noujeim 2003]), the composite right-left handed (CRLH) microstrip line ([Lim 2004-I,2004-II]), the microstrip logperiodic line [Augustin 2005], the half-mode microstrip line [Archbold 2010],[Ouegraogo 2011], and the half-mode substrate integrated waveguide (SIW) ([Suntives 2011, 2012]). Also, electronically tunable metasurfaces etched on grounded dielectric substrates have been employed for beam-scanning purposes in [Sievenpiper 2005]. Recently, a two-dimensional Fabry-Pérot LWA constituted by a ground plane and a tunable metasurface excited by a phased array has demonstrated electronic scanning of its pencil beam in the E-plane ([Ourir 2009], [Burokur 2013]). In [Debogovik 2014-II], a similar FP LWA is proposed to scan its pencil beam in the H-plane by controlling the phase difference applied to the small phased array employed to excite the FP cavity.

In this work, it is proposed a new configuration of electronically reconfigurable 1D LWA to achieve fixed-frequency beam steering, which is based on the passive (non-reconfigurable) 1D Fabry-Perot (FP) leaky-waveguides recently proposed in [García-Vigueras 2012-II]. The structure is composed of two periodic surfaces: a Partially Reflective Surface (PRS) and a High Impedance Surface (HIS). Varactors have been added to the HIS substrate in order to achieve electronic control of the pointing angle. Next, bases on general LWAs are introduced in Section 2.2. Later, in Section 2.3 it is reviewed the passive 1D FP LWA presented in [García-Vigueras 2012-II]. These concepts will be crucial to introduce the reconfigurable half-space electronicsteering FP LWA proposed in Section 2.4, and will ease the general comprehension of the starting hypothesis and the concepts demonstrated in this thesis. 


\subsection{Leaky-Wave Antennas}

LWAs can be classified inside a more general class of antennas, called travelling wave antennas ([Walter 1965]). These antennas basically employ the travelling waves which propagate through its structure as the main radiation mechanism. LWAs are capable of producing narrow beams, with the beamwidth limited by the size of the structure. The power of these waves is radiated, or "leaked", continuously as they propagate ([Oliner 2007]), so that they are commonly known as leaky waves. The principal advantage of LWAs is their simplicity, in terms of structure and excitation, and their high-gain radiation characteristics. The structure can be obtained from a simple type of open guide, for example, an open waveguide with a slot or periodic holes in the narrow wall ([Hansen 1940], [Hines 1957]), dielectric rods (e.g. [Peng 1978]), a microstrip line properly excited with the higher order mode (e.g. [Menzel 1979]), etc. Several types of LWA can be found detailed in [Oliner 2007]).

According to Marcuvitz ([Marcuvitz 1956]), who described for first time a theory which explained the nature of leaky waves, a leaky wave can be considered as a natural propagation mode (leaky mode) of an open guide. As any propagation mode, it can be characterized by a propagation constant, but due to the losses caused by the leakage of power which these waves suffers as they propagate, this constant will be always complex $\left(k_{z}=\beta-j \alpha\right)$, even if the guiding structure is lossless, consisting of both a phase constant $\beta$ and an attenuation constant $\alpha$ ([Jackson 2008]). The mechanism of radiation of a LWA is quite different from other types of travelling wave antennas and must not be confused. Fig. 2.2.1(a) represents a classical example of leaky-wave antenna. As previously explained, this antenna is characterized by a natural leaky wave which in this case is excited by the slot etched along the waveguide in the example ([Hansen 1940]). On the other hand, Fig. 2.2.1(b) shows a surface-wave antenna. These antennas support surface waves which propagate along the structure, but its radiation is not produced by the own dispersion nature of the wave, but due to the diffraction and refraction mechanisms caused at discontinuities, such as the presented at the end of a dielectric waveguide, as observed in the example. These antennas are usually more complex to characterize than leaky-wave antennas and require of a dielectric medium which allows the propagation of surface waves. Finally, another type of travelling wave antenna, the slotted waveguide antenna, is presented in Fig. 2.2.1(c). This antenna consists of an arrangement of resonant slots (approximated $\lambda / 2$ length) which couple energy from the mode of the rectangular waveguide to free space. Due to its resonant nature, these antennas are not properly considered as LWAs since leaky waves are not associated to resonant structures, although their radiation mechanism might be also explained from the leaky-wave theory viewpoint when these slots are becoming nonresonant ([Oliner 2007]). 


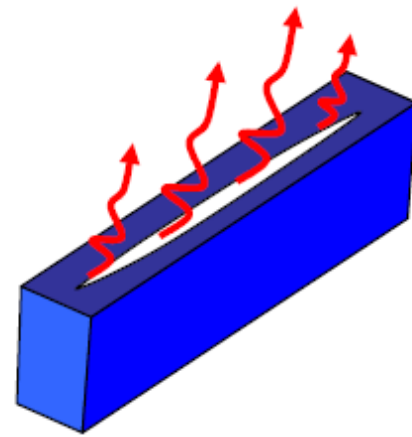

(a)

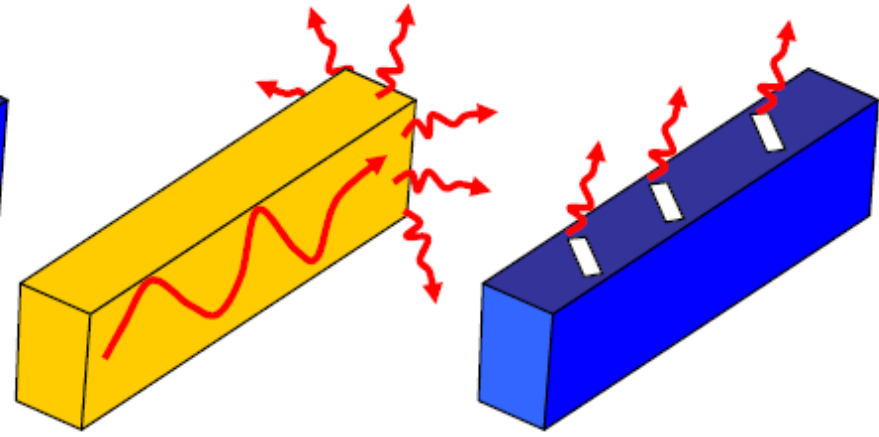

(b) (c)

Fig. 2.2.1 Types of travelling wave antennas. (a) Leaky-wave antenna. (b) Surface-wave antenna. (c) Slot-waveguide antenna (Fig. 2.18 in [Gómez 2006-I]).

As aforementioned, one important feature of LWAs is that the principal characteristics of the narrow beams produced by a LWA can be controlled by the leaky-mode propagation constant. As a general rule, it was observed that the beam angle $\left(\theta_{R A D}\right)$ of the pattern will be controlled by the phase constant, whereas the beamwidth $(\Delta \theta)$ is governed by the attenuation constant (also called leakage constant) of the leaky mode and the size of the antenna, as discussed below. Because of the relation between the phase constant with the pointing angle, and the inherent frequency dispersion which leaky waves usually suffer through the guiding-structure (as any propagation mode in a frequency-dependent transmission line) LWAs have the inherent property of frequency scanning, aforementioned in the previous section. However, it is worth to note that frequency scanning may turn into a double-edged sword for some fixed-frequency applications because it reduces the pattern bandwidth of the antenna (PBW). The PBW, which we will talk about more in detail in Section 2.9, can be defined from the frequency range over which the gain in a fixed observation direction is within $3 \mathrm{~dB}$ of that at the optimum frequency ([Balanis 2005]). The beam squint (measured in $\operatorname{deg} / \mathrm{Hz}$ ) of a LWA depends on the type of guiding structure employed ([Sievenpiper 2005]), being higher in partly dielectric-filled guides. In general, it has been observed that all common leaky-wave guides have a high beam squint, which reduces the PBW to a few percent. As can be thought, this issue represents one of the limitations of this type of antennas, which could be slightly mitigated with low-directive antennas with a wider beamwidth. In this sense, several works have analyzed this problem in detail to enhance the overall bandwidth of some types of LWAs ([Boutayeb 2007], [Bruni 2007], [Feresidis 2006], [Neto 2003], [Hosseini 2011], [Lovat 2006-II], [Mateo-Segura 2014], [Yuehe 2012], [Zhao 2005]), achieving wider bandwidth (10\%-15\% in X band) for antennas pointing at a fixed angle. However, this issue still exists for LWAs which require of simultaneous wideband, high-directive and fixed-frequency pattern-reconfigurable functionalities, such as long-range wideband communication systems. Nevertheless, many 
applications which operate at fixed frequency require of narrow band ([Bernhard 2005]), making LWAs very good candidates.

In next subsections, main concepts on LWAs are presented. In particular, in Section 2.2.1, the basic principles which govern physics of leaky waves and the radiation of LWAs are presented. In addition, some design equations employed in this thesis, and which allow a better comprehension of the concepts here explained, are also presented in this subsection. Later, in Section 2.2.3, general classification of LWAs is described, presenting some examples of complex transmission lines. Finally, it is introduced the category in which the reconfigurable FP LWAs presented in this thesis can be classified, according to its geometry and principle of radiation. 


\subsubsection{Principles of LWAs}

In order to introduce the principles and main relations which define LWAs, Fig. 2.2.2(a) depicts an example of a generic one-dimensional (1D) LWA which is supporting a leaky wave propagating along its longitudinal direction ( $y$-axis). In principle, it will be assumed that this LWA is dielectric-filled with a permittivity $\varepsilon_{\mathrm{r}}>1$ (greater than the air permittivity in free space) and will be considered narrow in the $x$-direction, so no propagation is produced along this axis, assuming a one-dimensional leaky-line oriented exclusively along the $y$-axis.

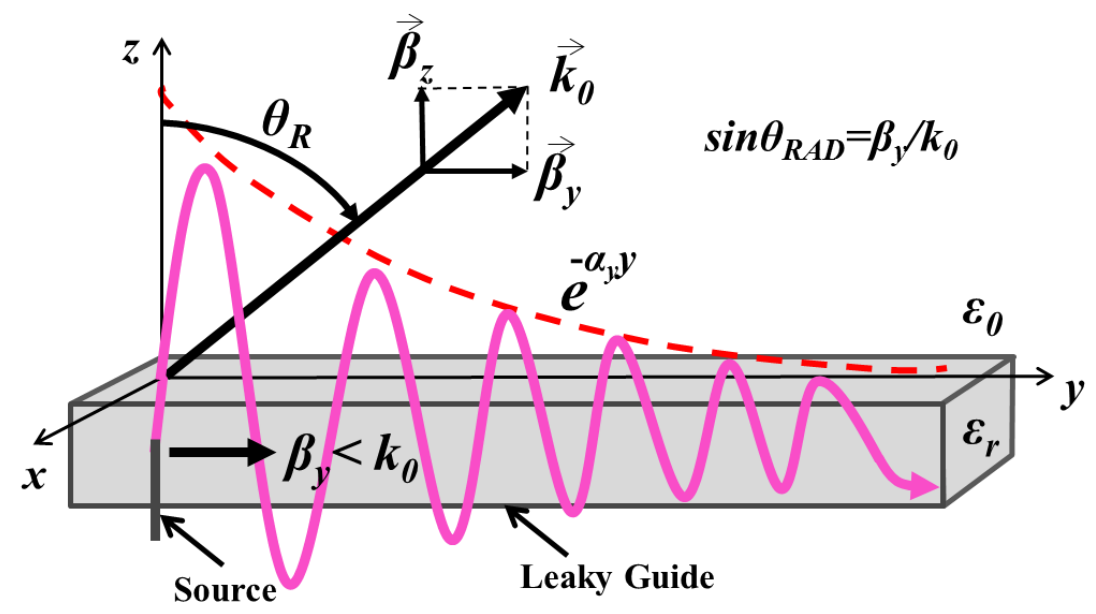

(a)

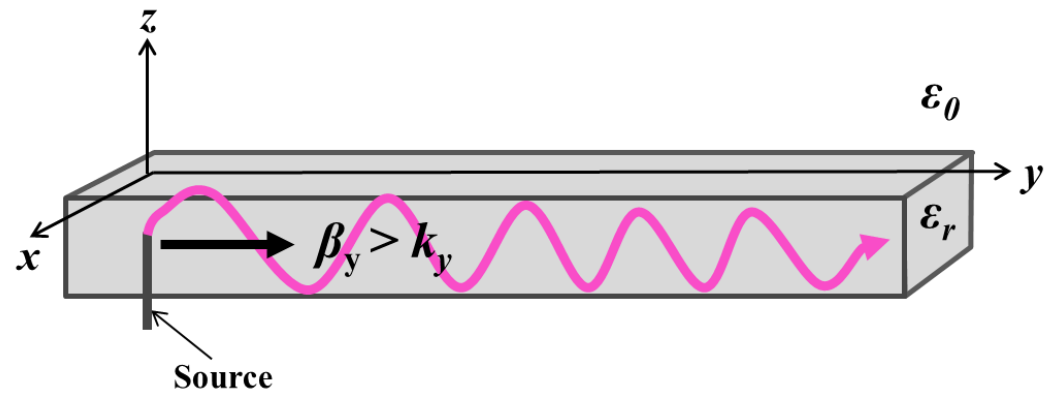

(b)

Fig. 2.2.2 Scheme of generic one-dimensional (1D) dielectric-filled guiding structure which supports a propagating (a) leaky wave and (b) a surface wave.

In this example, the complex propagation constant of the leaky wave is expressed as:

$$
k_{y}=\beta_{y}-j \alpha_{y}
$$

where the real part $\beta_{y}$ is the phase constant which defines the evolution of the wave phase along the guide in radians per meter $(\mathrm{rad} / \mathrm{m})$, and the imaginary part $\alpha_{y}$ is referred to the attenuation (leakage) constant related to the radiation losses per meter (nep/m) occurred along the antenna 
aperture (in addition to the ohmic losses due to conductors and/or dielectrics, which are assumed zero in a lossless case).

Assuming the case of a uniform (non-tapered) 1D LWA, any field component close to the aperture can be expressed as:

$$
\psi(x, y, z)=\psi_{0}(x, z) e^{-j k_{y} y}=\psi_{0}(x, z) e^{-j \beta_{y} y} e^{-\alpha_{y} y}
$$

From (2.2.2), it can be observed that the field distribution (the illumination) on the antenna will be determined by the longitudinal propagation constant $k_{y}$, being the phase shift defined by the phase constant $\left(\beta_{y}\right)$, whereas the amplitude decays exponentially as a function of the attenuation constant $\left(\alpha_{y}\right)$. Thereby, the far field (radiation pattern) generated by a LWA (which can be calculated in the usual fashion as the Fourier transform of the aperture field distribution) will be ultimately determined by the propagation constant of the leaky mode which illuminate the aperture. In this context, it is interesting to note that some of the most outstanding radiation pattern characteristics of a LWA, such its pointing angle $\left(\theta_{R A D}\right)$ or beamwidth $\left(\Delta \theta_{-3 d B}\right)$, can be approached from the phase and leakage constants of the leaky mode.

On one hand, the pointing angle, which defines the main radiation direction of the leaky wave, can be related to the longitudinal phase constant from a simple ray optics viewpoint. Observing Fig. 2.2.2(a), it is seen that such direction is defined by the longitudinal $\left(\beta_{y}\right)$ and vertical $\left(\beta_{z}\right)$ phase constants, which both together define the phase vector of the radiated wave in free space $\left(\vec{k}_{0}=\beta_{y} \hat{y}+\beta_{z} \hat{z}\right)$. Thus, the phase vector makes an angle, referred as the radiation (pointing) angle $\left(\theta_{R A D}\right)$ with respect to the $z$-axis defined by a simple trigonometric function ([Jackson 2008]):

$$
\tan \left(\theta_{R A D}\right)=\beta_{y} / \beta_{z}
$$

If the attenuation constant $\alpha_{y}$ is small $\left(\alpha_{y}<<\beta_{y}\right)$, the angle $\theta_{R A D}$ is given to a good approximation:

$$
\sin \left(\theta_{R A D}\right) \approx \beta_{y} / k_{0}
$$

with $k_{0}$ being the wavenumber of free space. Also it is worth noting that from this equation it can be easily derived that radiation from a leaky wave only can be produced if it is satisfied

$$
\left|\frac{\beta_{y}}{k_{0}}\right|<1
$$

This inequality is referred as the radiation condition and means that a leaky wave must be a fast wave, i.e. a wave with a phase velocity greater than speed of light, as stated below: 


$$
v_{p}=\frac{w}{\beta_{y}}=\frac{2 \pi f}{\beta_{y}}=\frac{2 \pi c_{0} / \lambda_{0}}{\beta_{y}}=\frac{c_{0}}{\beta_{y} / k_{0}}>c_{0} \Leftrightarrow\left|\frac{\beta_{y}}{k_{0}}\right|<1
$$

If Eq.(2.2.6) is not satisfied, the guide-structure stops behaving as a LWA, and starts supporting another type of travelling waves called surface waves, which propagate with a phase constant greater than the free space propagation constant $\left(\beta_{y}>k_{0}\right)$ (hence they are also called slow waves). A generic dielectric-filled guiding structure supporting a slow wave can be observed in Fig. 2.2.2(b). Given their slow nature, in general these waves will be only supported by partlydielectric filled structures which have materials with a dielectric permittivity greater than air $\left(\varepsilon_{\mathrm{r}}>1\right)$ in order to get phase velocities less than $c_{0}$. Thus, empty-filled waveguides will not be able to propagate them as we will show later. Although surface waves are not associated to a leakage as they propagate, as leaky waves, they also can produce radiation due to refraction and diffraction phenomena produced at discontinuities along the structure (such as bumps, imperfections, or discontinuity at the end of the line, as observed in Fig. 2.2.1(b)) ([Tamir 1963I], [Oliner 1979] [Oliner 2007], [Jackson 2008]).

On the other hand, the leakage constant $\alpha_{y}$ can be related with the beamwidth $\Delta \theta_{-3 d B}$ obtained by a LWA. From the simple structure of Fig. 2.2.2(a), and definition of the near fields in Eq.(2.2.2), it can be seen that the amplitude of the fields illuminating the LWA aperture decays as a function of $\alpha_{y}$ given the excitation of the example of Fig. 2.2.2(a). Also, it is well known that the directivity (beamwidth) of an antenna depends on its physical size. Hence, a large $\alpha_{y}$, which implies a large leakage rate and a short effective aperture length $\left(L_{A}\right)$ illumination, will produce a radiation beam with a large beamwidth. Conversely, a low $\alpha_{y}$ results in a long effective aperture and a narrow beam, provided the physical aperture is sufficiently long ([Oliner 1993]). Next equation verifies this relationship of the beamwidth with the effective aperture length and the leakage constant ([Oliner 2007]):

$$
\Delta \theta \approx \frac{1}{L_{A} / \lambda_{0} \cdot \cos \left(\theta_{R A D}\right)}
$$

where $\lambda_{0}$ is the wavelength of the operating frequency of the antenna. It is also worth to note that for a finite and fixed antenna aperture, if $\alpha_{y}$ is small, the beamwidth is determined primarily by the fixed aperture $L_{A}$, and $\alpha_{y}$ influences the beamwidth only secondarily. In such scenario, what will be affected strongly by the value of $\alpha_{y}$ is the efficiency of radiation $\left(\eta_{R A D}\right)$. The radiation efficiency of a LWA ([Goldstone 1959]) can be estimated as the total power radiated over the total power in the antenna, and it is also related to $\alpha_{y}$ as:

$$
\eta_{R A D}=1-e^{2 \alpha_{y} L_{A}}
$$


The LWA aperture length is usually designed for a radiation efficiency $\eta_{R A D}=0.9$, so that $90 \%$ of the energy must be radiated and the remaining $10 \%$ absorbed by a matched load placed at the end of the guide ([Oliner 2007]). In such a case, it can be approximated from Eq.(2.2.8) that ([Oliner 2007]):

$$
L_{A} / \lambda_{0} \approx \frac{0.183}{\alpha_{y} / k_{0}}
$$

which demonstrates the inverse relationship between the leakage constant $\alpha_{y}$ and the effective aperture length $L_{A}$. Finally, substituting Eq.(2.2.9) in Eq.(2.2.7) a direct relation between $\alpha_{y}$ and $\Delta \theta$ is obtained:

$$
\Delta \theta \approx \frac{\alpha_{y} / k_{0}}{0.183 \cdot \cos \left(\theta_{R A D}\right)}
$$

From this equation it is readily observed that high-directive antennas (low beamwidth) can be achieved by a low value of $\alpha_{y}$ and the proper aperture length.

Eq.(2.2.4-2.2.10) can be considered some of the principal equations to deal with the design of LWAs. These equations allow us to predict in a simple and accurate way some of the main characteristics of the radiation pattern and efficiency of the antenna, what is also another attractive of LWAs, since it does not require of heavy computational cost, what is very useful from a practical point of view. However, for such predictions it is inherently necessary to know the propagation constant of the supported leaky modes by a dispersion analysis of the open structure. Several techniques have been developed to compute this dispersion for different open structures ([Walter 1965], [Hessel 1969], [Tamir 1956-I, II, 1969], [Menzel 1979], [Oliner 1979], [Oliner 2007]). Thus, the prediction of the dispersion diagrams is quite important to analyze how the leaky-mode propagation constant varies as a function of frequency or some physical parameter of interest for the design of the antenna structure, what will also be of great interest for the design of the structures presented here, as will see. In addition, it is worth to note that other interesting advantage of LWAs is given precisely by the guiding structure of the antenna, which can allow in many occasions some type of physical modulation or tapering technique, to control the leaky-mode propagation constant, and hence the field distribution of the aperture with a higher degree of freedom ([Oliner 2007]). In general, the aperture field of a uniform LWA with strictly uniform geometry (with no physical variations along the propagation direction) has an exponential decay (usually slow) of the fields' amplitude, generating a radiation pattern with sidelobe level poor. However, other LWAs present a nonuniform (tapered) structure ([Oliner 19569], [Burghignoli 2003], [Gómez 2011-I], [GarcíaVigueras 2012-II], [Maci 2011], [Martínez-Ros 2012-I, 2013-I]...) along its guiding structure, modulating the phase and/or attenuation constant of the leaky wave as it propagates by 
dispersion engineering techniques. Thus, tapered LWAs in different technologies have been conceived to reduce side lobe level (e.g. [Honey 1956], [Maci 2011], [Minatti 2011] [Oliner 1959]...), enhance aperture efficiency (e.g. [García-Vigueras 2012-II]), or conceive other more exotic devices, such as conformal antennas (e.g. [Gómez 2011-II], [Losito 2007]), focusing lenses (e.g. [Monnai 2011], [Martinez-Ros 2013-II], [Gómez 2013-II]), broadbeam antennas ([Gómez 2011-III]), null-pointed antennas (e.g. [Gómez 2010]), etc. 


\subsubsection{Frequency Dispersion of LWAs}

In general, for any LWA, both $\beta_{y}$ and $\alpha_{y}$ are functions dependent on frequency $\left(\beta_{y}(w), \alpha_{y}(w)\right)$. From the dispersion-diagram response of the classical LWA presented in Fig. 2.2.3, this dependence is evidenced and different propagation regimes can be distinguished. The definition of cutoff, radiation and bounded regimes of a leaky-wave antenna can be found in books such as [Oliner 2007], or other interesting papers such as [Tamir 1963-I], [Oliner 1979, 1986-I and II, 1987], [Bagby 1993], [Lin 1997] or [Gomez 2006-III]. These references lead to the following background knowledge necessary to work with leaky waves. As an illustrative example, Fig. 2.2.3 shows the evolution with frequency of the normalized phase and leakage constants $\left(\beta_{y} / k_{0}\right.$ and $\alpha_{y} / k_{0}$, respectively) which correspond to the first mode propagated by a one-dimensional dielectric-loaded LWA such as presented in Fig. 2.2.2(a). For the characterization of the different propagation regimes we will consider the lossless case (ideal dielectric materials and metals).

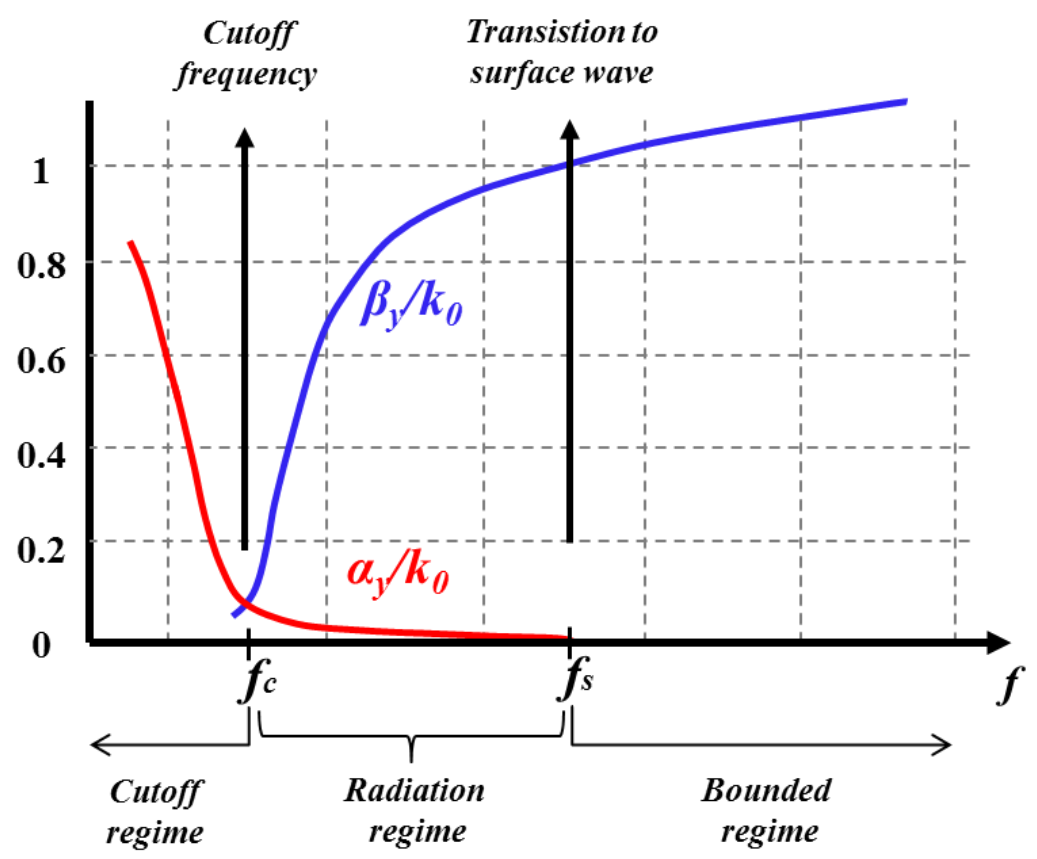

Fig. 2.2.3 Example of classical frequency-dispersion diagram in a uniform 1D dielectric-filled LWA. Leaky-mode propagation regimes.

The cutoff frequency of the leaky mode is located where $\beta_{y}=\alpha_{y}$, that in the example corresponds to $f_{c}$. At this point it is considered that the leaky mode starts propagating through the structure. The leaky wave turns into a surface wave when $\beta_{y} / k_{0}=1$ at $f_{s}$ (where the bounded regime starts). Between these two important frequencies (marked in Fig. 2.2.3), the radiation regime is located. Here, the radiation condition Eq.(2.2.5) is satisfied, and thus, the leaky-mode energy is leaked to 
the angle $\theta_{R A D}$ (related to the phase constant by Eq.2.2.4), which in principle can be scanned from nearly $0^{\circ}$ to $90^{\circ}$, hence the frequency scanning property of LWAs ([Oliner 2007]). The minimum value of $\theta_{R A D}$ is produced at the cutoff frequency, which is normally near broadside $\left(\theta_{R A D} \approx 0^{\circ}\right)$. As previously explained, the bounded regime is characterized by zero leakage $\left(\alpha_{y} / k_{0}=0\right)$, in coherence with the solid red line in Fig. 2.2.3 (considering a lossless case), and so, no leaky-wave radiation is here produced. Note that in a lossy case, $\alpha_{y}$ will not be null in this region, but it accounts just for the ohmic losses of materials, but not for radiation losses. However, the cutoff regime (also named reactive region) is characterized by values of $\alpha_{y}$ greater than $\beta_{y}$. This sharp increment of $\alpha_{y}$ below the cutoff frequency does not represent an increase of the radiation rate of the leaky wave, but an increment in the reactive rate caused by the attenuation of the fields at the waveguide input, since no leaky modes can be supported by the guide below such frequency ([Gomez 2005-I], [Lin 1997], [Oliner 2007]).

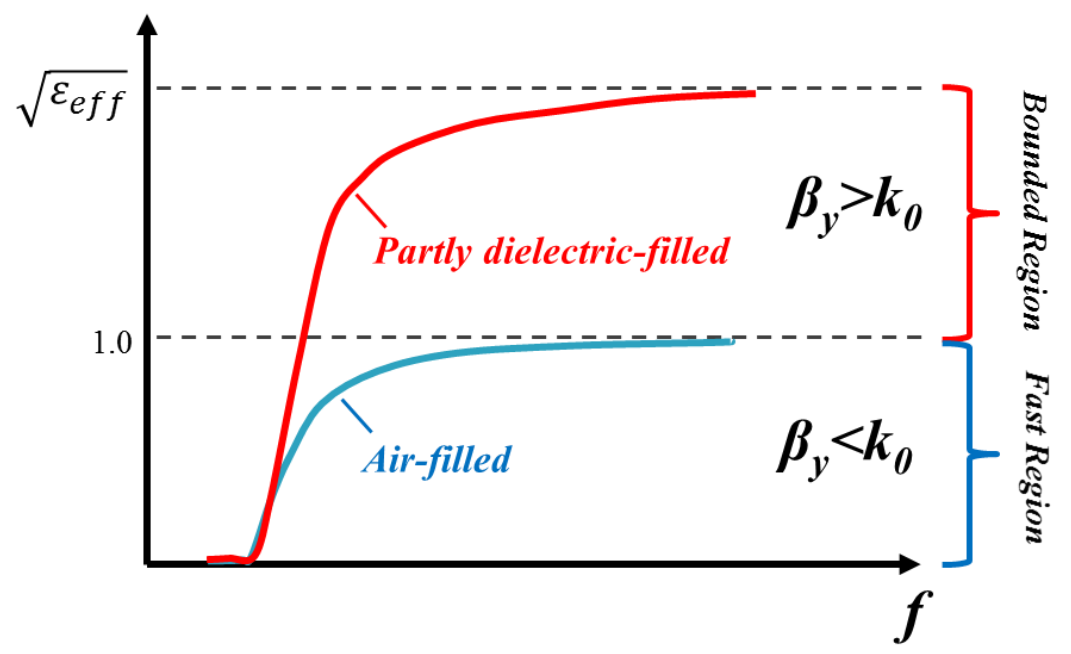

Fig. 2.2.4 Comparison between dispersion in a partly dielectric-filled waveguide LWA and an air-filled empty waveguide LWA (Fig. 11.3 in [Oliner 2007]).

In the case of air-filled guides, this scanning behavior is similar in principle but differ somewhat. When an empty-filled guiding structure is employed, no surface-wave transition is possible, since both free space and waveguide mediums have the same permittivity, and so no air-dielectric interface exists to support the propagation of surface waves, as aforementioned ([Oliner 2007]). This is qualitatively shown in Fig. 2.2.4, where the classical dispersion of an empty waveguide (blue solid line) and a partly dielectric-filled waveguide (red solid line) are shown. It is observed that when the waveguide is air-filled, as the frequency is increased, the normalized phase constant tends to one, but never reaching this value. However, partly dielectric-filled guides exceed this point, becoming to an asymptotic dispersion diagram bounded by the square root of the dielectric permittivity of the medium $\left(\beta_{y} / k_{0}=\sqrt{\varepsilon_{e f f}}\right)$. Therefore, it can be concluded that LWA configurations based on a conventional empty 
waveguide will be dominated by fast waves relative to the free-space velocity, avoiding slow waves can arise, what will limit the maximum scanning range at endfire angles (close to $90^{\circ}$ ). 


\subsubsection{Classification of LWAs}

LWAs can be categorized according to different factors; the most common are the geometry and principle of operation ([Jackson 2008], [Oliner 2007]). According to the geometry, we can classify LWAs as a function of the structure type: uniform or periodic, which in turn can also be classified as one-dimensional (1D) LWAs or two-dimensional (2D) LWAs.

The principal distinction between a 1D and a 2D LWA falls on the antenna aperture electrical dimensions. As previously explained, the far field radiation pattern form produced, will be a function of the aperture size and also on the field distribution with which the leaky wave illuminates the antenna aperture.

An example of 1D LWA is presented in Fig. 2.2.5. In this case, the longitudinal direction of the antenna is oriented along the $y$-axis, while it is electrically short in the orthogonal plane. An ideal $x$-polarized source excites the structure from one end. In this case, the leaky wave will propagate through the longitudinal direction, giving a natural exponential distribution field on the aperture (as was also shown in the example presented in Fig. 2.2.2(a)). Thus, due to this antenna is electrically long in the $y$ direction, and short in the orthogonal one, a fan beam is produced, narrow in the H-plane ( $Y Z$ plane) and wide in the E-plane. The principal cut planes of a typical fan beam can be observed in Fig. 2.2.6. In this case, the beam is narrow in the H-plane, given the long electrical dimension of the structure in the $y$ direction. It is worth to highlight that the wide beam obtained in the orthogonal plane can be narrower (till generate a pencil beam) as the width of the antenna (in the direction perpendicular to the propagation; $x$-direction in this example) is increased ([Honey 1959]).

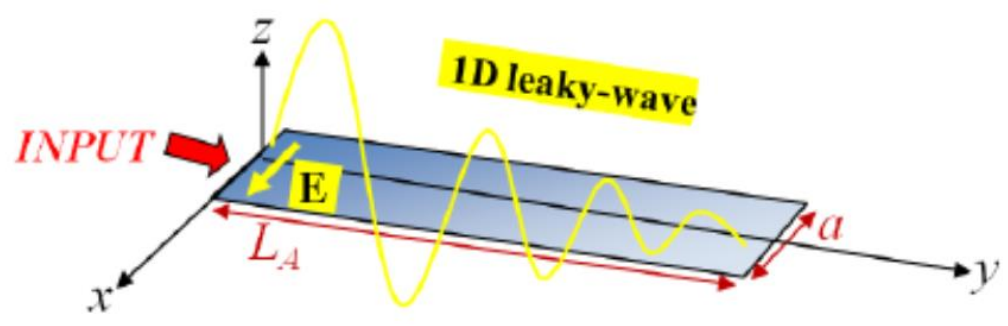

Fig. 2.2.5 Scheme of 1D LWA excited from one end (Fig. 3.1.6 in [García-Vigueras 2012-II]).

Secondly, a 2D LWA is one where the guiding structure itself has a two-dimensional surface. These structures usually support a cylindrical leaky wave that propagates outward in the radial direction from the source or feed ([Jackson 2008], [Oliner 2007]), as can be observed in the scheme of Fig. 2.2.7. In principle, this type of LWA is capable of producing pencil beams pointing at broadside, or conical beams when the antenna is pointed at a higher angle, with the cone axis perpendicular to the aperture plane, as illustrated in Fig. 2.2.8(a) and (b) respectively. 
Also, examples of the principal cutting planes of a pencil beam and a conical beam are depicted in Fig. 2.2.9 and Fig. 2.2.10 respectively.

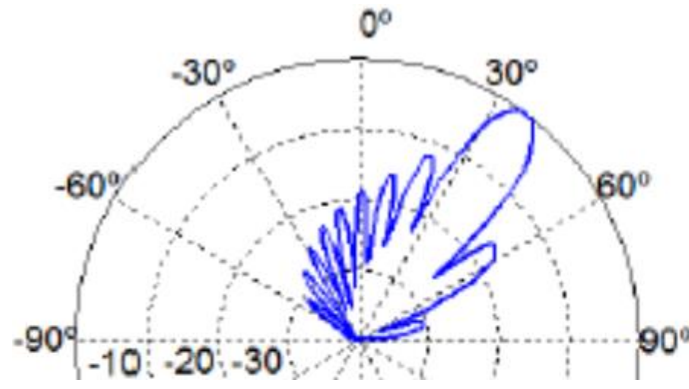

(a)

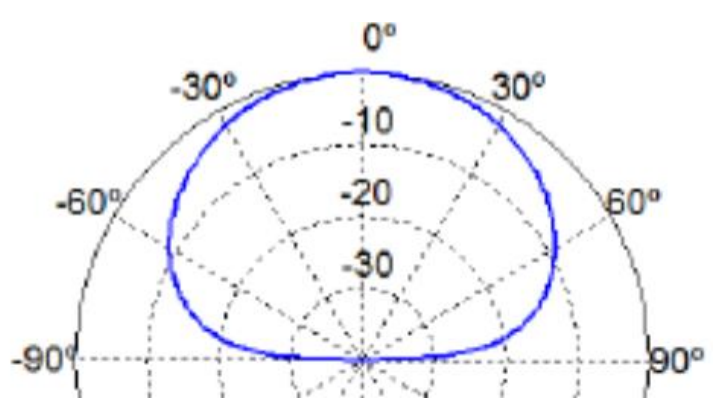

(b)

Fig. 2.2.6 Normalized radiation pattern in $\mathrm{dB}$ of 1D LWA with dimensions: $\mathrm{a}=0.5 \lambda_{0}, L_{A}=6 \lambda_{0}$, fed at one end $\left(k_{y} / k_{0}=0.6+j 0.01\right)$. (a) H-plane $(Y Z)$, (b) E-plane (XZ) (Fig. 3.1.7 in [GarcíaVigueras 2012-II]).

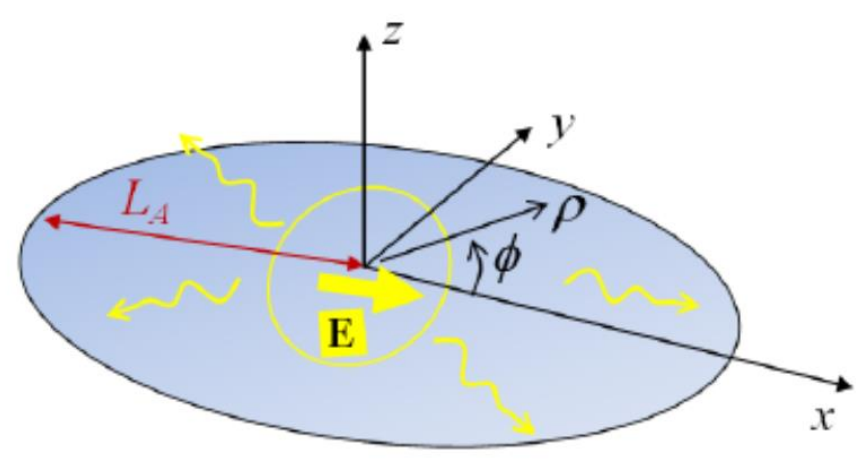

Fig. 2.2.7 Scheme of 2D LWA excited from the center (Fig. 3.1.10 in [García-Vigueras 2012II]).

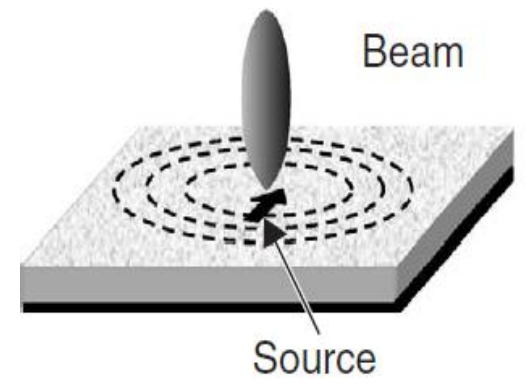

(a)

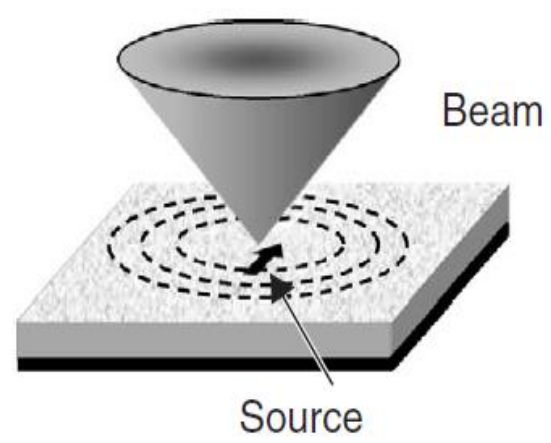

(b)

Fig. 2.2.8 Illustration of the type of beams produced by a 2D FP LWA: (a) a broadside pencil beam and (b) a symmetric conical beam at an angle $\theta_{0}$ (Fig. 7.5 in [Jackson 2008]).

Also, LWAs can be classified as a function of its geometry. In this category, the first defined type is the uniform LWA. Some examples of uniform 1D LWAs are presented in Fig. 2.2.11(a) and (b). A typical example of uniform 1D LWA is an air-filled waveguide with a slot along its 
narrow wall, as presented in Fig. 2.2.11 (a). Also, a simple microstrip line excited by its higher order mode $\left(\mathrm{EH}_{1}\right)$ can be included in this category (Fig. 2.2.11(b)). A 2D uniform Fabry-Pérot (FP) LWA is presented in Fig. 2.2.11(c), which top layer is composed by a flat stack of multiple dielectric slabs.

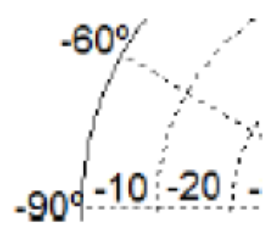

(a)

(b)

Fig. 2.2.9 Pencil beam pointing at broadside produced by a 2D LWA $\left(L_{A}=6 \lambda_{0}\right)\left(k_{y} / k_{0}=\right.$ $0.1+\mathrm{j} 0.1$ ). Normalized radiation pattern in $\mathrm{dB}$ of (a) H-plane (YZ), (b) E-plane (XZ) (Fig. 3.1.11 in [García-Vigueras 2012-II]).

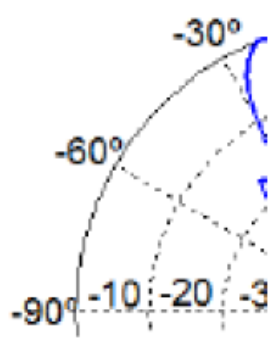

(a)

(b)

Fig. 2.2.10 Conical beam produced by a 2D LWA $\left(L_{A}=6 \lambda_{0}\right)\left(k_{y} / k_{0}=0.39+\mathrm{j} 0.015\right)$. Normalized radiation pattern in $\mathrm{dB}$ of 2D LWA $\left(L_{A}=6 \lambda_{0}\right)$. (a) H-plane (YZ), (b) E-plane (XZ) (Fig. 3.1.12 in [García-Vigueras 2012-II]).

As observed from these examples, the geometry of a uniform structure is kept constant along its longitudinal direction of propagation of the leaky wave ( $y$ axis, in Fig. 2.2.11(a) and (b), $x$ and $y$ in Fig. 2.2.11(c)). It is worth to note that, for classification purposes, in this category can be also included the aforementioned tapered LWAs (antennas with a modulation of its aperture along its length to control the beam-form). 


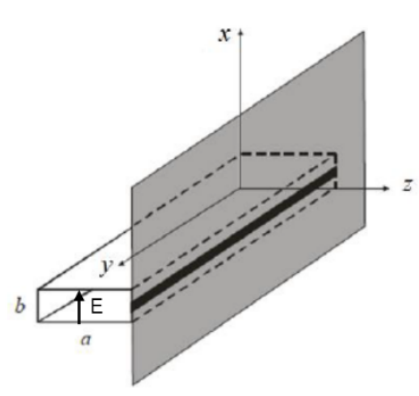

(a)

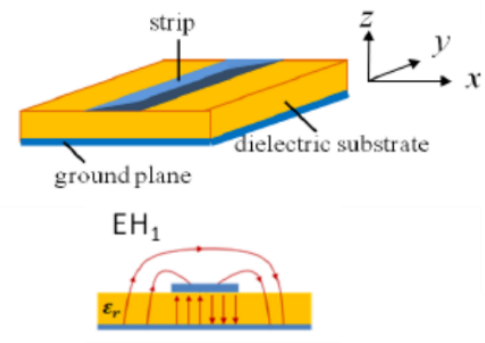

(b)

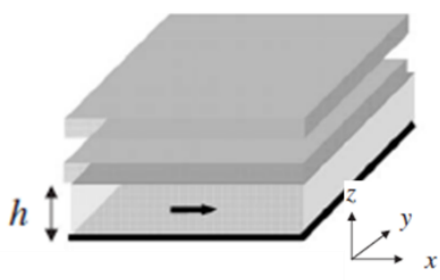

(c)

Fig. 2.2.11 Example of uniform LWAs: (a) Slot waveguide ([Hansen 1940]), (b) Higher order mode microstrip LWA ([Menzel 1979]) and (d) 2D FP LWA with a stack of dielectric layers

([Jackson 1993]).

Uniform LWAs are usually associated to a radiation principle based on fundamental model of the open guide. For example, in Fig. 2.2.11(a), the waveguide fundamental $\mathrm{TE}_{10}$ mode is a fast wave, with $\beta<k_{0}$. In particular, to a good approximation (assuming that the slot does not affect the phase constant significantly),

$$
\beta=\sqrt{k_{0}^{2}-k_{c}^{2}}
$$

Where $k_{c}=\pi / \mathrm{a}$. The radiation causes the wavenumber $k_{z}$ of the propagation mode within the (now open) waveguide structure to become complex $k_{z}=\beta-j \alpha$. Thus, it can be concluded that uniform structures will be usually associated to the radiation of the fundamental mode, which can become leaky, by different radiation mechanisms ([Gómez 2005-I], [Jackson 2008], [Oliner 2007]).

The second type of geometry considered for a LWA is periodic geometry. These structures can be obtained for example applying arrays of holes on a waveguide or arrays of strips on a grounded dielectric slab, as presented in Fig. 2.2.12(a) and (b), respectively. Also metamaterial LWAs are constructed by periodic unit cells, as observed in Fig. 2.2.12(c). Finally, a doublelayer (PRS-HIS) 1D FP LWA or a 2D FP LWA composed of a parallel plate with a top metallic plate substituted by an array of patches (a frequency selective surface) can be also included by its periodic geometry (Fig. 2.2.12(d) and (e) respectively). However, periodic-geometry LWAs have been sub-classified in two different groups, according to the radiation principles employed to control their radiation characteristics. Following the nomenclature employed in other important reference books ([Jackson 2008], [Oliner 2007]), these antennas can be categorized as "periodic" (in terms of periodicity-based) and "quasi-uniform" LWAs. 


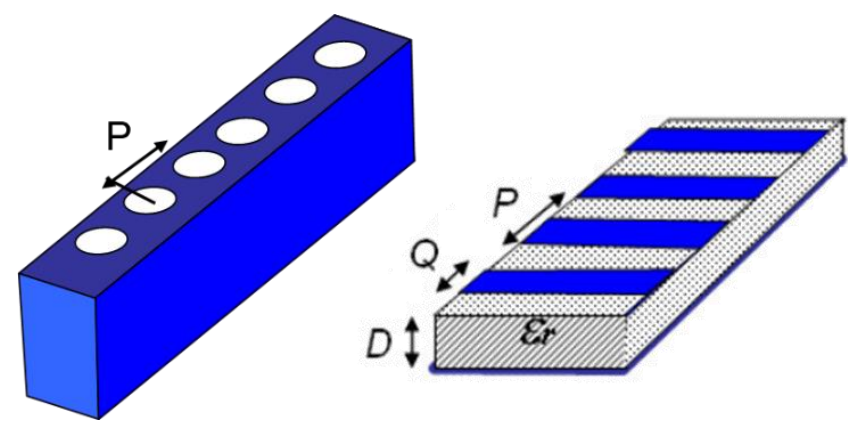

(a) (b)

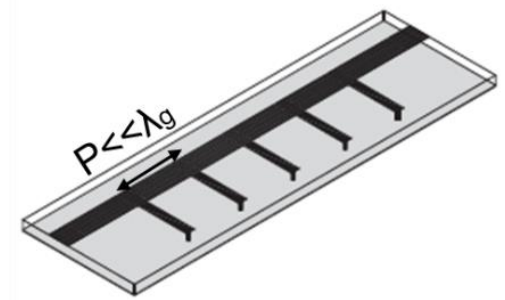

(c)

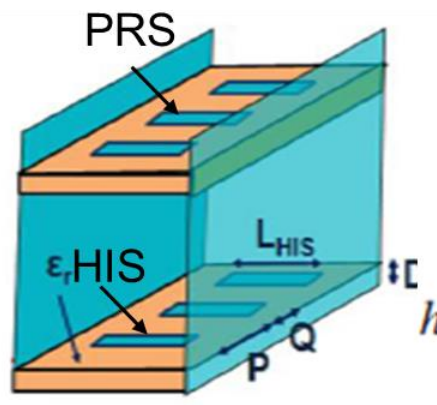

(d)

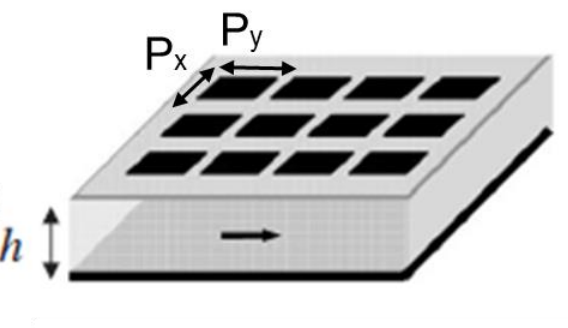

(e)

Fig. 2.2.12 Example of periodic LWAs: (a) Holey waveguide ([Hines 1957]), (b) Dielectric rod with metal strips, (c) LWA CRLH microstrip line ([Caloz 2005]), (d) Double-layer (PRS-HIS)

1D FP LWA ([García-Vigueras 2012-II]) and (e) 2D FP LWA composed of a dielectric slab with an array of patches ([Zhao 2005-II]).

First one, as the name suggests, is based on the periodicity of the structure. An important difference between uniform and periodic LWAs is that the dominant mode on the former is fast $\left(\beta / k_{0}<1\right)$, while the rest are slow $\left(\beta / k_{0}>1\right)$. This principle could be applied to the case of the dielectric-filled holey waveguide presented in Fig. 2.2.12(a) or the dielectric rod loaded with an inductive grid depicted in Fig. 2.2.12(b). Due to periodicity, an infinity of space harmonics ([Walter 1965], [Oliner 2007]) can be considered in the structure, which contribute to the total aperture field. Some of these harmonics may be fast, while the rest are slow; the fast space harmonics will radiate. Each one can be characterized by a wavenumber $k_{n}=\beta_{n}$-j $\alpha$. The relation between the harmonics phase constants (normalized) is

$$
\beta_{n} / k_{0}=\beta_{0} / k_{0}+n \frac{2 \pi}{P}
$$

where $n$ is an integer number $(n= \pm 1, \pm 2 \ldots), k_{0}$ the propagation constant in free space at the frequency of operation, $\beta_{0} / k_{0}$ is the normalized phase constant associated to the $\mathrm{n}=0$ fundamental harmonic and $\mathrm{P}$ is the LWA periodicity. An example of the classical dispersion curves presented by a periodic LWA is shown in Fig. 2.2.13, where it has been plotted the phase constant associated to the $n=0, n=-1$ and $n=-2$ harmonics versus frequency. In this figure it is marked the 
frequencies at which each harmonic starts radiating (where $\left|\beta_{n} / k_{0}\right|<1$, points $\mathrm{A}, \mathrm{B}$ and $\mathrm{D}$ ), and the frequencies at which they become surface waves $\left(\left|\beta_{n} / k_{0}\right|<1\right.$, points $\mathrm{C}$ and $\left.\mathrm{E}\right)$. The first aspect that should be now highlighted is that, in contrast to the previous examples of non-periodic LWAs, backward radiation can be now produced by the harmonics with $n<0$, due to the fact that they present a negative phase constant during a certain frequency range (as it has been marked in Fig. 2.2.13 in the case of the $n=-1$ harmonic). Thus, LWAs based in the radiation of the negative higher order harmonics can scan from backward to forward angles, in contraposition to uniform LWAs based on the fundamental mode, which are only able to scan at forward angles. Another relevant aspect is that radiation from more than one harmonic can be produced at certain frequencies (for example, $n=0$ and $n=-1$ radiate at different angles in the range $[B, C]$ ); appearing the so called grating lobes regimes, where more than one main beam arise due to each space harmonic operating in the radiation (fast) zone. Single radiation from the fundamental harmonic can be assured if the radiation of the $n=-1$ harmonic starts at a frequency higher than the one at which the $n=0$ harmonic becomes a surface wave (i.e., if $B$ occurs at higher frequency than C). The proximity between the dispersion curves may be controlled by changing the value of $\mathrm{P}$. Therefore, single radiation from the fundamental harmonic can be obtained if it is satisfied that $\mathrm{P}<\lambda_{0}$ (with $\lambda_{0}$ the wavelength at the operating frequency $k_{0}=2 \pi f / \lambda_{0}$ ). This condition is highly important to properly design the structure, assuring that the required space harmonic is radiating to the desired radiation angle. Therefore, periodic LWAs based on its periodicity are usually dielectric or partly dielectric-filled guiding structures, which are operating with the fundamental space harmonic in its slow-wave region and a periodicity which allows to operate with the $n=-1$ higher order space harmonic in the fast region for scanning from backward to forward angles, which is one of the principal advantages of these antennas.

The second principle is based in the radiation from the fundamental harmonic $\left(\beta_{0}\right)$ of the periodic LWA, similar to the employed by uniform structures. For this reason, the structures presented below are also categorized as quasi-uniform LWAs. As will see below, these structures employ periodicities less than the wavelength, to avoid radiation from high order harmonics (as previously explained). In this category, two types of periodic LWAs can be included: metamaterial LWAs and Fabry-Pérot (FP) LWAs comprised by periodic surfaces.

Metamaterials, or so called Composite Right-Left Handed (CRLH) materials, unlike natural Right-Handed (RH) lines, can present a negative phase constant since they can be characterized by a simultaneous negative permittivity and permeability $(\varepsilon<0$ and $\mu<0)$, as was described in [Veselago 1986]. CRLH property is achieved by periodic loadings on a host (RH) transmission line which introduce series capacitances $\left(C_{s}\right)$ and parallel inductances $\left(L_{p}\right)$ to incorporate the $\mathrm{LH}$ response. The term "Left-Handed" (LH), refers to the fact that the electric field, magnetic field, and phase vectors build a left-handed triad, instead of the regular right-handed ("RH") response, 
which materials present in nature. This simultaneous $\mathrm{LH}$ and $\mathrm{RH}$ response grants to this structures to create LWAs that are able to scan from backward to forward with the fundamental mode of the structure, without exciting higher order harmonics, as occurred with the previous periodic LWAs ([Caloz 2005]). At present, CRLH materials have not been found available in nature, so that they have to be synthesized by homogeneous structures composed of a combination of discrete unit cells (which size is much smaller than the guided wavelength at the frequencies of interest) ([Caloz 2005], [Eleftheriades 2005]). An example of typical CRLH LWA is presented in Fig. 2.2.12(c) ([Caloz 2005]).

Fig. 2.2.13 Dispersion in a periodic LWA (substrate with permittivity $\varepsilon_{r}$ ) (Fig. 3.1.16 in [GarcíaVigueras 2012-II]).

Finally, FP LWAs (Fig. 2.2.12(d) and (e)), can also have a periodic geometry when the reflective surfaces which comprise the structures (the FP cavities) are constituted by some type of periodic surface, such as an array of scatterers (frequency selective surfaces (FSSs)) and/or a High Impedance Surface (HIS), as will see in next sections. These antennas are based neither higher order space harmonics (the periodic reflecting surfaces are designed to be smaller than the wavelength, and avoid radiation from higher-order harmonics) nor LH properties. In this case, these structures operate with the resonance of the fundamental mode of the resonant FP cavity in order to originate leaky-wave radiation. In next sections, we will focus on this type of periodic structures and will detail their radiation principle and design considerations. In particular, the structure and operation principles of the passive (non-reconfigurable) 1D FP LWA presented in Fig. 2.2.12(d) will be reviewed further in detail in Section 2.3, and will be investigated to incorporate beam-steering reconfiguration, as will be explained in Section 2.4. 


\subsection{Passive 1D FP LWA}

Recently, a new contribution in the field of FP LWAs was presented by García-Vigueras in her thesis ([García-Vigueras 2012-II]). In that work it was aimed for first time the efficient synthesis of tapered FP LWAs. To do so, they firstly focused on the double-layered FP antenna proposed in [Feresidis 2005]; both periodic layers (PRS and HIS) were studied and modulated in order to synthesize any aperture illumination by tapering techniques. The achievement of this goal lead to design 1D and 2D FP LWAs with high aperture efficiency, while assuring high radiation efficiency to minimize diffraction and standing waves (in particular best results demonstrated radiation and aperture efficiency rates over $80 \%$ for a tapered 2D FP LWA). In order to achieve the previous main objective, several specific steps were followed progressively. Firstly, the periodic layers that form the PRS and HIS of the FP LWA were properly characterized. It is extremely important that these layers are accurately modeled by equivalent pseudo-analytical closed-form expressions that can be later employed for the characterization of the whole FP structure. Secondly, the analysis and design of uniform FP LWA was carried out by a useful tool which was developed, based on the Transverse Resonance Method (TRM), for the efficient and accurate analysis of these structures. A complete dispersion analysis of the uniform structure was performed in order to find the way to control the propagation of the constituent leaky waves. Thirdly, modulated (tapered) FP LWAs were synthesized. In the second and third steps, the one-dimensional scenario was firstly considered as an approximation of the two-dimensional counterpart.

In this thesis, we focus on the initial double-layer passive (non-reconfigurable) 1D FP LWA which was proposed in [García-Vigueras 2012-II]. The principal characteristic of this antenna is its structure simplicity and the possibility of synthesizing almost any type of aperture distribution by the proper design of the dipole-based frequency selective surfaces (FSSs) which compose the PRS and HIS layers which comprise the FP cavity. The double-layer structure of the reconfigurable antenna proposed in this chapter, and the ones presented in next ones, will be based on the radiation principles of this structure. For this reason, this section is dedicated to present this passive 1D structure and principal conclusions derived from [García-Vigueras 2012-II]. Next, a brief overview on FP LWAs history and state of the arte is firstly given, in order to contextualize this work. 


\subsubsection{Fabry-Pérot LWAs}

The structures of the reconfigurable antennas presented in this thesis are based on a particular type of LWA popularly known in literature as Fabry-Pérot (FP) cavity LWA ([García-Vigueras 2012-II], [Liu 2009], [Jackson 2008], [Oliner 2007]). The general structure of a FP LWA is usually based on a resonant cavity (also called FP cavity) comprised between a Partially Reflective Surface (PRS) and a grounded substrate, as depicted in Fig. 2.3.1. This configuration is excited by an embedded low-gain radiator which may be realized in practice in a variety of ways, with some examples being a dipole, a microstrip patch on the ground plane, a slot on the ground plane, or a waveguide-fed aperture in the ground plane. This simple single-feed system has allowed to obtain high gain antennas with relative low complexity and low volume, what makes FP LWAs an attractive solution for certain applications when compared to externally fed antennas (such as reflectors, lenses or reflectarrays), or phased arrays, which need much more complicated feeding networks.

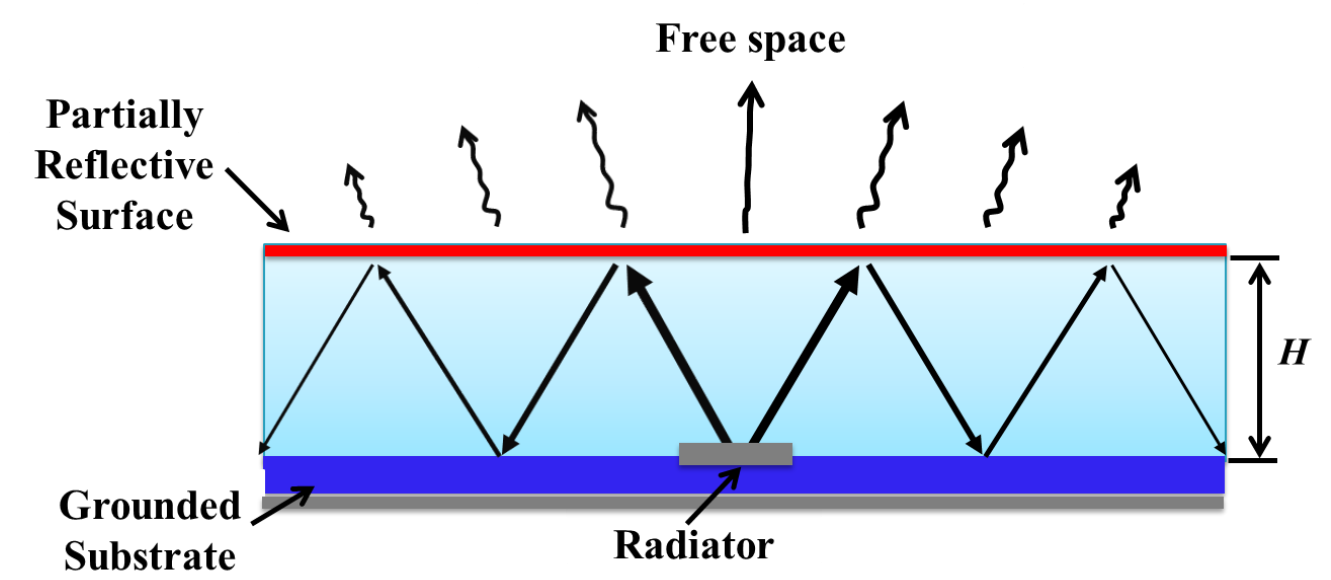

Fig. 2.3.1 Schematic diagram of a classical Fabry-Pérot LWA excited from the centre.

FP LWAs were firstly introduced by Trentini in 1956 ([Trentini 1956]), who discovered the increase in directivity by placing a Partially Reflective Surface (PRS) in front of a waveguide aperture antenna with a reflecting screen (see the original antenna scheme in Fig. 2.3.2(a) and the designed prototypes in Fig. 2.3.2(b)). Trentini baptized this antenna as a "reflex-cavity antenna" due to the resonant cavity comprised between the radiator ground plane and the top PRS. However, the analysis of the structure was not originally developed from a leaky-wave theory viewpoint, but from an optical ray approach. Indeed, these antennas were not originally recognized as being LWAs and were originally explained from different starting points and focuses. The term "Fabry-Pérot" was coined when these structures were explained in terms of Fabry-Pérot resonances in the optics field, due to its analogy to the classical FP structure, where the waves are bounced forth and back between two partially reflective mirrors ([Jackson 2008]), 
as observed in Fig. 2.3.1. Even at present, it is usual that technical works on this topic employ the simplicity of this approach to explain their radiation mechanism in a simple way or as a quick design guideline to obtain the initial dimensions of the FP cavity ([Feresidis 2005], [Goussetis 2006], [Weily 2008]), but employ a leaky-wave theory viewpoint for final design analysis. Also, investigations on 2D FP LWAs to enhance directivity of FP LWAs were conducted in the 1980s ([Alexopoulos 1984], [Jackson 1985], [Yang 1987]). These structures employed EBG structures as PRSs which consisted of stacks of high permittivity dielectric layers. In this case, these structures were explained in terms of Electromagnetic Band-Gap (EBG) effects ([Akalin 2002], [Jackson 1985, 1988, 1993], [Weily 2005-I, 2005-II, 2006, 2007I, 2007-II], [Yang 1987]), so that these particular type of FP structures are more commonly known today as EBG resonator antennas. The analysis of this type of structure as a LWA was done by Jackson and Oliner in the late 1980s and early 1990s ([Jackson 1988], [Jackson 1993]). FP resonances or EBGs provide useful insights, although an explanation in terms of leaky waves provides the most physically fundamental way to explain their operation ([Jackson 2008]).
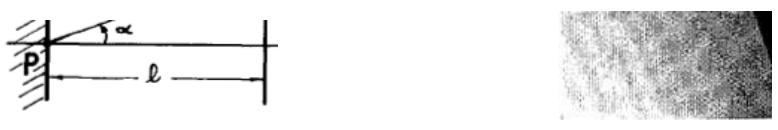

(a)

(b)

Fig. 2.3.2 (a) Original antenna scheme of multiple reflections between reflector and PRS and (b) PRS-loaded designed antennas (Figs. 1 and 7 in [Trentini 1956]).

Today, it has been widely accepted that these structures can be treated as a type of LWA ([Liu 2009]), (being also referred in the literature as FP LWAs ([García-Vigueras 2012-II])) and their performance obeys to the fundamental relations described in earlier sections of this chapter ([Walter 1965], [Hessel 1969], [Tamir 1969], [Oliner 2007]). According to the aforementioned classification, these antennas can be classified as uniform or periodic, according to its geometry, which depends on the type of reflecting surfaces which constitute the FP cavity. In any case, 
these antennas are usually based on the resonance of the fundamental mode excited in the cavity, being treated as uniform or quasi-uniform LWAs, in terms of radiation principle. Thereby, it is understood that the waves launched by the source become leaky modes [Oliner 2007] of the resonant cavity due to the leakage allowed by the top PRS. This can be qualitatively understood from a ray optics viewpoint, as observed in Fig. 2.3.1, where leaky waves are represented as rays bouncing off in the FP cavity. This way, energy gradually escapes (leaks) as the leaky wave travels across the structure. Thereby, with a proper design of the structure, leaky waves illuminate a large radiating area and consequently, an increase of directivity of the primary low-gain source is achieved. It is worth to note that, as any resonator, the height $(H)$ of the FP cavity will be a determinant factor to tune its resonant frequency, and hence the central operating frequency of the antenna. At present, because of the effect of PRS or the FP resonator which characterize these structures, these FP LWAs are also commonly called either PRS antennas (e.g. in [Weily 2008]) or FP resonator (FPR) antennas ([Liu 2009]).

The PRS of a FP LWA can be either composed by a uniform layer (e.g. a superstrate EBG structure composed of a stack of dielectric layers as proposed in [Alexopoulos 1984]) or a periodic FSS composed of some type of periodic arrangement, such as dipoles, patches, slots, holes, etc. ([Jackson 2008], [Trentini 1957]). 1D or 2D FP LWAs can be implemented with 1D or 2D PRS respectively. The original work of Trentini introduced the concept of 2D FP LWAs, implementing different types of two-dimensional PRSs, such as a stack of closely spaced wires or strips (see Fig. 2.3.2(b)). Same strip-based PRS was used by Honey in his early work ([Honey 1959]). In Honey's work, however, the PRS was linearly arranged in one dimension and the structure fed from one end, making Honey's structure the first type of 1D FP LWA. As introduced in the past section, 1D LWAs can produce in principle a fan beam, narrow in one plane and wide in the other. However, as Honey described, if the width of the 1D antenna (perpendicular to the direction of propagation) is sufficiently large, the 1D structure can also produce a pencil beam, but the beam is steerable only in one plane, and the beam scan limited to the forward quadrant (unless the structure is excited from both ends) ([Jackson 2008]). On the other hand, 2D FP LWAs are illuminated by a cylindrical leaky wave that propagates outward in the radial direction from the central feed and produces a pencil beam (narrow in both the Eand H-planes) pointing at broadside, or a conical beam with the cone axis perpendicular to the aperture plane, as illustrated in Fig. 2.2.8 (a) and (b), respectively. A 2D FP LWA provides a simple mean to obtain a narrow pencil beam at broadside, using a simple source, what is an advantage in terms of structure design and cost, in contrast to $2 \mathrm{D}$ phased arrays. However, the pattern bandwidth of the structure can be limited, since the beam will evolve into a conical beam as the frequency increases to scan at higher angles, resulting in a loss of gain in the 
broadside direction. This limits its applicability to systems which do not require of high directivity, wide bandwidth or scanning requirements.

As observed, FSSs play a key role in the conception of new FP LWAs. At present, FSSs represent one of the most investigated topics in the field of engineering and physics due to the high amount of applications that they have, such as for the synthesis of microwave and optical filters/resonators or enhancement of antennas. In general, a FSS is any constructed periodic surface which possesses some kind of filtering properties for plane waves. Typical FSSs, such as the one shown in (a)

Fig. 2.3.3(a), usually consist of a doubly periodic arrangement of planar metallic scatterers (or its dual structure, periodic arrays of apertures made in a thin metallic surface). Many different shapes of the FSS elements have been proposed, some of them are shown in (a)

(b)

Fig. 2.3.3(b), the election depends on the FSS application.

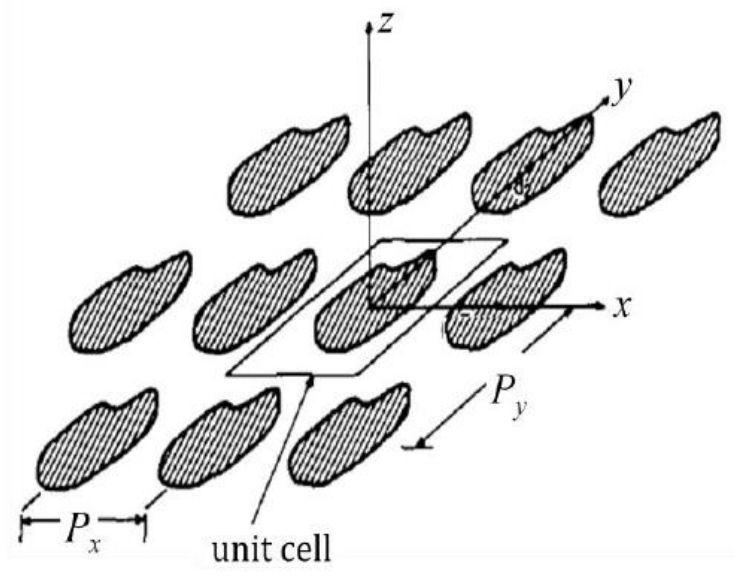

(a)

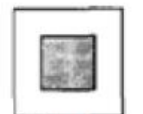

(1)

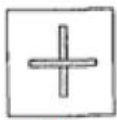

(4)

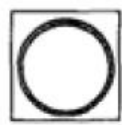

(7)

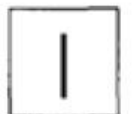

(2)

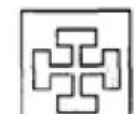

(5)

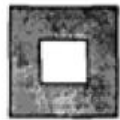

(8)

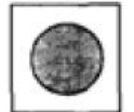

(3)

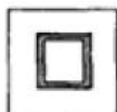

(6)

(b)

Fig. 2.3.3 (a) Scheme of a patch-based free-standing FSS. (b) Some typical FSS unit cell geometries: (1) square patch, (2) dipole, (3) circular patch, (4) cross dipole, (5) Jerusalem cross, (6) square loop, (7) circular loop, (8) square aperture (Figs. 1 and 5 in [Mittra 1988]).

The transmission and reflection coefficients for these surfaces are dependent on the frequency of operation and usually also depend on the polarization and the angle of incidence with which the electromagnetic wave strikes the FSS surface. The fields scattered by a FSS will exhibit resonances of different nature as a function of the relative dimensions of the scatterers and periodicity with the frequency of the impinging wave. In a conventional operating regime, where the scatterers of the FSS are comparable to the wavelength of the frequency of the impinging wave, these surfaces may exhibit generally a total reflection (patches) or total 
transmission (apertures) when the size of the scatterer is comparable to an integer number of half wavelength of the frequency of the impinging wave. To achieve this FSS operation, the size and shape of the array elements are tailored in order to control the characteristics of this first resonance, also referred as conventional resonance. If the excitation frequency is augmented, the FSS periodicity becomes greater than the wavelength with respect to the incident wave frequency and the apparition of grating lobes can be found ([Mittra 1988]). Then, the FSS can be referred as a diffraction grating. Also, another phenomena which can present FSSs with subwavelength scatterers and periodicities comparable to the wavelength, are extraordinary transmission/reflection peaks before the onset of the first grating lobe ([Ebbessen 1998], [Beruete 2004, 2005 and 2007-I], [Azad 2005], [García-Vigueras 2012-I, II]). These resonances are considered "extraordinary" because they are not directly related to the electrical length of the FSS scatterers, but to the periodicity of the array. Nevertheless, at present, most of the application in the microwave frequency range which employ FSSs, usually design structures comparable to the wavelength which are commonly operated around their conventional resonance.

The applicability of FSS in the microwave antenna field was initiated at the beginning of the XX century, when G. Marconi and C. S. Franklin patented the first FSS structure applied to the microwave region, to act as a parabolic reflector of wire sections for wireless telegraphy ([Marconi 1919]). In [Trentini 1956], FSS were employed to design different types of PRS to comprise the aforementioned FP LWAs (arrays of metallic patches, a metallic plate perforated periodically with holes, a one-dimensional array of metallic wires or strips (see Fig. 2.3.2(b)). A few years later O'Nians employed again FSS to enhance the efficiency of reflector antennas [O’Nians 1966]. Also, boosted by the military applications, B. A. Munk and others developed interesting work on periodic surfaces in the middle of the 60's and 70's ([Munk 2000]). More recently, in the microwave field, investigations have combined FSS with guiding structures to modify their scattering properties, changing the propagation of waves along them and achieving diffraction characteristics such as frequency stop-bands, pass-bands or band-gaps [Sievenpiper 1999]. These structures are classified under the broad terminology of "Electromagnetic Bandgap (EBG)" structures. In this scenario, also, new structures have been invented with interesting properties, such as quasi TEM propagation in rectangular waveguides [Seager 1999], [Yang 1999], size reduction [Goussetis 2007-II], [Caiazzo 2004] or dispersion compensation in waveguides [Goussetis 2007-I]. In [Sievenpiper 1999], a new type of textured surface with interesting EBG properties was conceived by placing a patch-based FSS with vias connected to a ground plane. This structure, usually referred as High Impedance Surface (HIS), has the interesting property of presenting a reflection coefficient with a reflection phase that can be flexibly varied as a function of the FSS dimensions. In particular, one of the most characteristics 
operating regimes of a HIS is when it presents a reflection phase of zero degrees, behaving as an Artificial Magnetic Conductor (AMC). Recently, other types of HIS (with a more simplistic structure) have been presented, such as a grounded dielectric slab with a patch-based FSS without vias ([Goussetis 2006-I]). HISs have been employed for multitude of applications in microwave field. For example, absorbers have been conceived employing low-efficiency HIS substrates which dissipate energy from impinging waves to avoid reflection and diffraction phenomena ([Costa 2010], [Engheta 2002], [Tretyakov 2003-II]). Latest advances include also planar polarizers which allow to filter or change electronically the polarization of an incident wave ([Doumanis 2012, 2014]). In the antenna field, an increasing interest on FSS, and periodic structures in general, has also arisen. In particular, last interests have been focused on the analysis of new HIS, EBG substrates, hard and soft electromagnetic surfaces, PRSs, as well as Left-Handed (LH) ([Caiazzo 2004]) metamaterials. Some examples of HIS applicability can be found in [Sievenpiper 1999] or [Shumpert 1999], where the EBG properties of these structures have been proposed to suppress non-desired surface waves or enhance the bandwidth of low profile antennas. Also, they have been applied to attain new types of antennas, apart from FP LWAs. For example, textured surface LWAs ([Sievenpiper 2005]), metamaterial LWAs ([Liu 2002], [Lim 2004-I, II]...) or reflectarray/transmitarray antennas ([Huan 2008]).

After Trentini, the applicability of FSS in the FP LWA field decayed till Feresidis proposed in 2001 a PRS structure based on a doubly periodic arrangement of printed-circuit scatterers ([Feresidis 2001]). Till that time, investigation on uniform 2D FP LWAs, employing dielectric layers as PRS (the aforementioned EBG resonator antennas) were being mainly studied ([Alexopoulos 1984], [Jackson 1985], [Yang 1987], [Thevenot 1999]). However, one disadvantage of these antennas was the higher thickness required by the stacks of dielectrics slabs, what could be substituted by an FSS composed of a thin array of metallic scatterers. Other similar FSS configurations were later proposed in [Zhao 2005-II and III], [Gardelli 2006], [Guérin 2006] and [Boutayeb 2006]. By that time, a general explanation of the working mechanism of PRS printed resonators was given in [Zhao 2005-I, II]. The same year, it was proposed to replace the bottom grounded substrate by a HIS to reduce the cavity height [Feresidis 2005], and hence the profile of the FP cavity. Later, FP LWAs based on a 2D wire medium were presented in [Lovat 2006-III, IV] and [Burghignoli 2008] with interesting dispersion properties in the E- and $\mathrm{H}$ planes. Recently, many other works can be found in the literature which exploit other types of FP structures with enhanced bandwidth ([Bruni 2007], [Feresidis 2006], [Weily 2007], [Yuehe 2012], [Mateo-Segura 2014]), dual band ([DuranSindreu 2013], [Kelly 2007], [Moghadas 2013]) or dual/circular polarizations ([Moghadas 2013], [Orr 2014], [Debogovic 2014-I]). Finally, electronic reconfiguration capabilities have been also achieved with multi-band operation ([Burokur 2013] [Ourir 2007], [Weily 2008]), 
electronic beam-steering ([Burokur 2013], [Costa 2011], [Debogovic 2014-I, II], [Edalati 2011], [Ourir 2009]) or electronic beam-shaping ([Edalati 2009], [Debogovic 2010, 2014-I]). In this latter field, most reconfigurable FP LWAs based its reconfiguration mechanisms on a tunable FSS which changes its reflection properties to reconfigure the FP cavity. For example, in [Ourir 2007, 2009] and [Burokur 2013], an electronically metamaterial PRS loaded with varactors is employed. [Weily 2008] presents a novel 2D FP LWA with an electronically-tunable HIS with phase-agility to change the central operating frequency of the antenna. As explained below, on this latter work is inspired the steering mechanism principles of the antennas proposed in this thesis. 


\subsubsection{Double-layer 1D FP LWA}

In Fig. 2.3.4, it is depicted a schematic of the passive 1D FP LWA presented in [GarcíaVigueras 2012-II]. The antenna is implemented in hybrid technology ([Gómez 2005-I], [GarcíaVigueras 2011-II]), which combines metallic waveguides and printed circuit boards (PCBs). In particular, the structure of the 1D FP LWA consists of a parallel-plate waveguide (PPW) loaded with two PCBs: a top partially reflecting surface (PRS) and a bottom high impedance surface (HIS) (see Fig. 2.3.4 (a)). In the case of the HIS, the substrate is back-shielded by a ground plane to achieve the special AMC response which characterizes HIS structures ([Sievenpiper 1999]). Both PCBs are formed by FSS constituted by one-dimensional periodic arrays of resonant metallic patches printed on thin dielectric substrates. The two PCBs are separated at a distance $H$, comprising a one-dimensional FP resonant cavity which allows the propagation of a perturbed $\mathrm{TE}_{01}$ leaky mode. The dimensions of the structure (described in the caption of Fig. 2.3.4) were obtained to operate on an operating frequency band around $15 \mathrm{GHz}$.

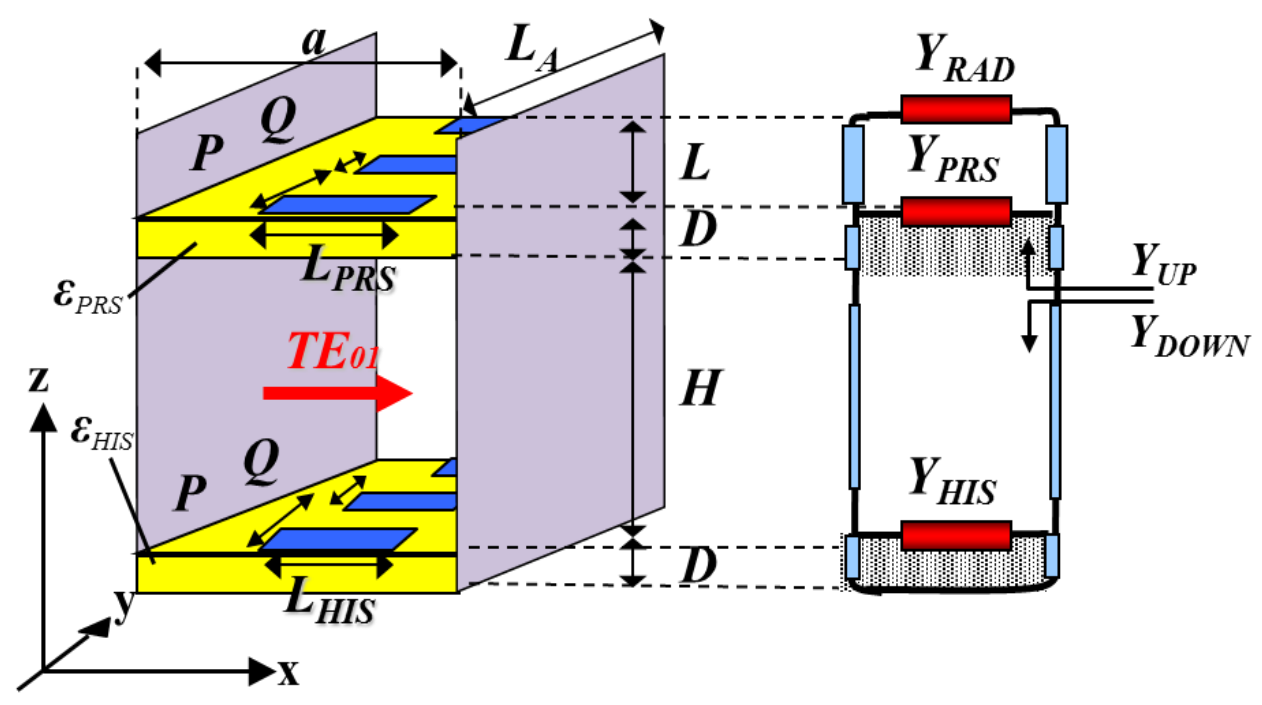

(a)

(b)

Fig. 2.3.4 (a) Original configuration of the passive 1D Fabry-Pérot LWA and (b) Transverse Equivalent Network proposed in [García-Vigueras 2012-II]. ( $a=H=11 \mathrm{~mm}, S=5 \mathrm{~mm}, D=1.13 \mathrm{~mm}$, $\varepsilon_{P R S}=\varepsilon_{H I S}=2.2, L_{P R S}=9 \mathrm{~mm}, L_{H I S}=9 \mathrm{~mm}, P=1.5 \mathrm{~mm}, Q=0.5 \mathrm{~mm}$ ) (Fig. 3.2.1 in [García-Vigueras 2012-II]).

As demonstrated in [García-Vigueras 2011-II], this double-layer 1D (PRS-HIS) FP LWA allows the leakage rate to be adjusted by changing the resonant length of the PRS patches ( $L_{P R S}$ in Fig. 2.4.1), while the resonant length of the HIS patches ( $L_{H I S}$ in Fig. 2.4.1) controls the pointing angle at a fixed design frequency. This is well described in the next subsections, where 
a general review on the effect of the HIS and PRS dipoles length is given from a dispersion analysis viewpoint.

A modal analysis of the periodic structure of Fig. 2.3.4 was performed employing a full-wave Method of Moments technique employed by the modal analysis tool P.A.M.E.L.A ([Gómez 2006-II]).

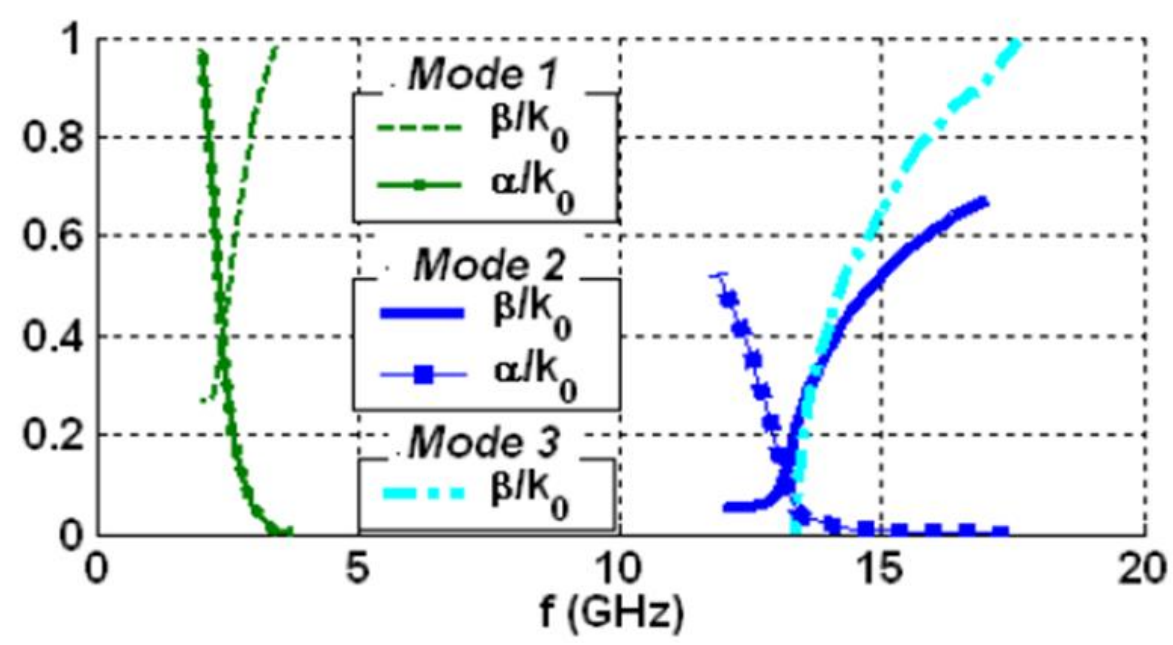

(a)
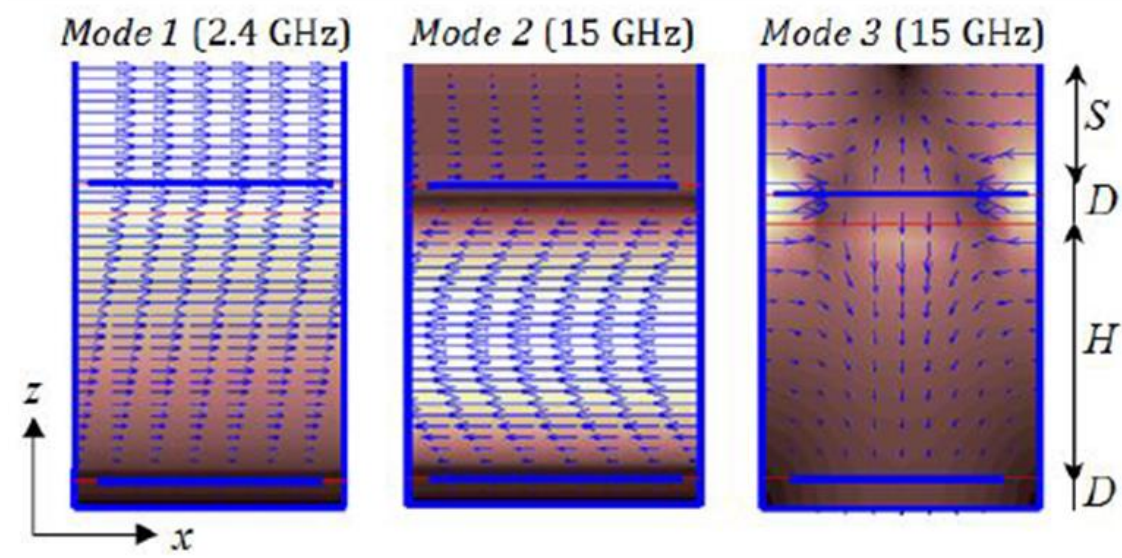

(b)

Fig. 2.3.5 (a) Dispersion of natural modes in the 1D FP LWA $\left(L_{P R S}=L_{H I S}=10 \mathrm{~mm}\right)$ (b)

Transverse electric fields in the cross-section of the LWA for each mode (Fig. 3.2.2 in [GarcíaVigueras 2012-II]).

The leaky-mode dispersion results for the case $L_{P R S}=L_{H I S}=10 \mathrm{~mm}$ are plotted in Fig. 2.3.5. From this study, it can be concluded that three different modes are present in the structure: the horizontally polarized channel-guide mode supported by the PPW (mode 1 in Fig. 2.3.5), and the perturbed horizontal $\mathrm{TE}_{01}$ and vertical $\mathrm{TE}_{10}$ modes of the cavity (modes 2 and 3 in Fig. 2.3.5). In the operating band ( $15 \mathrm{GHz}$ ), only modes 2 and 3 are in the fast-wave regime, while mode 1 is a non-radiant slow-wave ([Oliner 2007]). Due to the symmetry of the structure, the 
vertically polarized mode 3 does not leak power to free space ([Gómez 2005-I, II, 2006-I, II]). The proposed LWA has been designed to operate with the perturbed $\mathrm{TE}_{01}$ leaky-mode (mode 2 in Fig. 2.3.5(b)), with an electric field directed along the $x$ axis in Fig. 2.3.4 in the operating frequency band. Thus, single TE mode operation will be assured by using a horizontally polarized feeding. To this aim, a rectangular waveguide will be operated with the classical $\mathrm{TE}_{01}$ mode of these guiding structures, to excite the FP structure. This 1D FP LWA will be excited from one end, so one fan beam will be generated with scanning capability in one plane. The elevation radiation angle $\theta_{R A D}$ is defined in the H-plane ( $Z Y$ plane in Fig. 2.3.4) and it is measured with respect to the $z$-axis. The dispersion characteristics of the structure will be calculated considering TE polarization in the $Z Y$ plane.

The injected $\mathrm{TE}_{01}$ mode is perturbed by the dipoles of the PRS and the HIS, creating a leaky mode which bounced back and forth between the two sheets, propagating through the guiding structure in the $y$-direction. The dispersion of this leaky mode can be analyzed with a specific tool, which was also developed in [García-Vigueras 2012-II], that is based on the Transverse Resonance Method (TRM) and the LWA Transverse Equivalent Network (TEN) shown in Fig. 2.3.4(b), so that the complex propagation constant $k_{y}\left(k_{y}=\beta_{y}-\mathrm{j} \alpha_{y}\right)$ is obtained for the desired frequency and dimensions of the structure, by an efficient (in terms of computational time) and accurate way, as will see. All the leaky-wave dispersion results presented in next subsections have been obtained from this simple TEN, and they are validated with a Finite Element Method (FEM) full-wave simulator ([HFSS 2011]). The TRM will be also employed in the methodology proposed for the analysis and design of the reconfigurable FP LWAs proposed in this thesis, and will be explained in Section 2.6.

Next, the effect of $L_{P R S}$ and $L_{H I S}$ on the pointing angle $\theta_{R A D}$ and normalized leakage rate $\alpha_{y} / k_{0}$, studied in detail in [García-Vigueras 2012-II], are reviewed. The conclusions derived from these studies will be essential for the future comprehension of the principles which govern the radiation characteristics of the reconfigurable LWAs presented later. Also, in this chapter it is demonstrated how the synthesis of different illuminations can be performed with this doublelayer 1D FP LWA to obtain antennas with different radiation patterns. 


\subsubsection{Effect of the PRS}

The transparency of the PRS determines the amount of energy that reaches the top aperture of the LWA, thus controlling the leakage rate. Fig. 2.3.6 shows the leaky-mode frequency dispersion curves for different lengths of the PRS dipoles (the other dimensions of the LWA are summarized in the caption of Fig. 2.3.4). Fig. 2.3.6 shows the frequency response of the radiation angle (obtained from normalized phase constant applying Eq.(2.2.4)), and the normalized leakage rate $\left(\alpha_{3} / k_{0}\right)$ for a double-layer (PRS-HIS) 1D LWA, with different values of $L_{P R S}\left(L_{P R S}=7,8,9 \mathrm{~mm}\right)$ and a fixed HIS $\left(L_{H I S}=9 \mathrm{~mm}\right)$. From these curves, it is evidenced how the resonant length of the dipoles of the PRS ( $\left.L_{P R S}\right)$ has a minimal effect on the pointing angle dispersion curves $\left(\theta_{R A D}\right)$, but it has a strong influence on the $\alpha_{y} / k_{0}$. It is also shown that the analytical results agree well with the simulated ones using FEM ([HFSS 2011]) shown in circles in the figure.

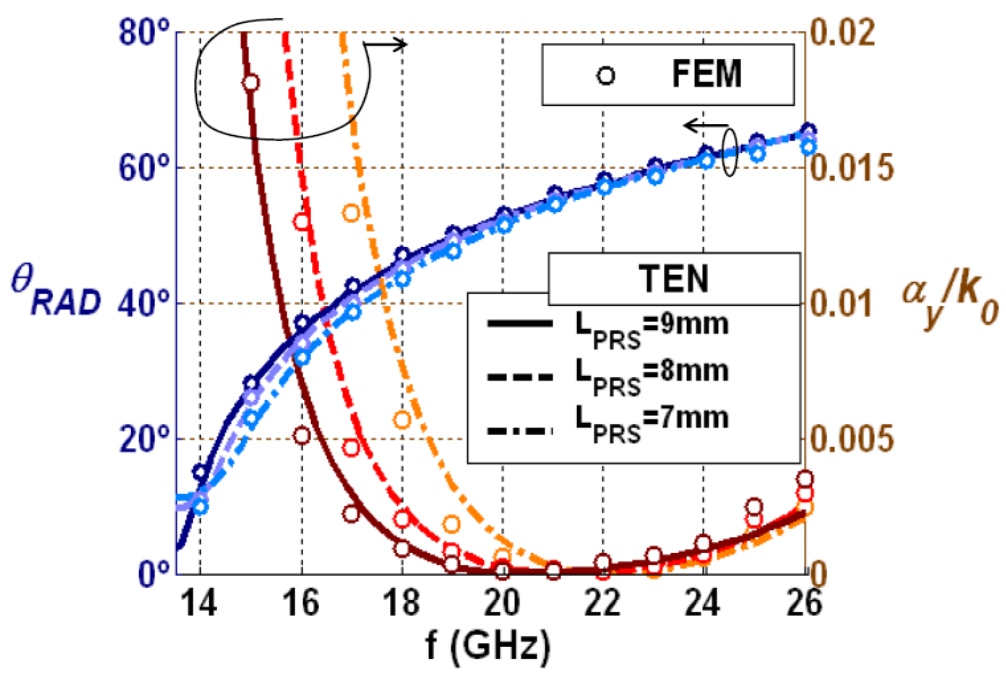

Fig. 2.3.6 Leaky-mode frequency dispersion curves for the 1D FP LWA in Fig. 2.3.4(a) for different values of $L_{P R S}\left(L_{H I S}=9 \mathrm{~mm}\right)$ (Fig. 3.2.3 in [García-Vigueras 2012-II]).

This response is equal to the conventional behavior (frequency scanning) of LWAs, due to the inherent dispersion nature of leaky-waves ([Oliner 2007]). However, the peculiarity of this airfilled LWA is found near $20 \mathrm{GHz}$, where the radiation rate becomes zero $\left(\alpha_{y}=0\right)$, although the bounded regime has not been reached yet (it can be seen that $\beta_{y} / k_{0}<1$ and the pointing angle $\left.\theta_{R A D}=\operatorname{asin}\left(\beta_{y} / k_{0}\right)<90^{\circ}\right)$. Then, after this null-radiation point, it seems that the radiation rate increases for higher frequencies. The physics underlying this non-conventional behavior of the radiation rate is related to the resonant nature of the top dipole-based PRS. In particular, the null radiation frequency is found when the dipoles at the PRS resonate. This feature is related to the resonant nature of the top PRS. In Fig. 2.3.7, it is illustrated four different configurations of the proposed LWA $\left(L_{P R S}=[4,6,8,10] \mathrm{mm}\right.$, with constant $\left.L_{H I S}=9 \mathrm{~mm}\right)$. An additional case has been 
added, considering the 1D LWA with a metallic plate (PEC) replacing the top PRS, remaining a closed waveguide (keeping inside a HIS with $L_{H I S}=9 \mathrm{~mm}$ ). Fig. 2.3.7(a) shows the reflection coefficient under the PRS ( $\rho_{P R S}$, see Fig. 2.3.4(b)) associated to each of the studied configurations, while Fig. 2.3.7(b) shows the frequency dispersion diagrams. When the dipoles in the PRS resonate, this surface behaves as a PEC and $\left|\rho_{P R S}\right|=1$. Therefore, for each of the studied cases the resonant frequency is located at the point in which their curves of $\rho_{P R S}$ match the red curve in Fig. 2.3.7 (and thus, $\rho_{P R S}=1$ is satisfied). Consequently, at these exact frequencies, the $\beta_{y} / k_{0}$ curves associated to each case match the red curve in Fig. 2.3.7, and $\alpha_{y}=0$.

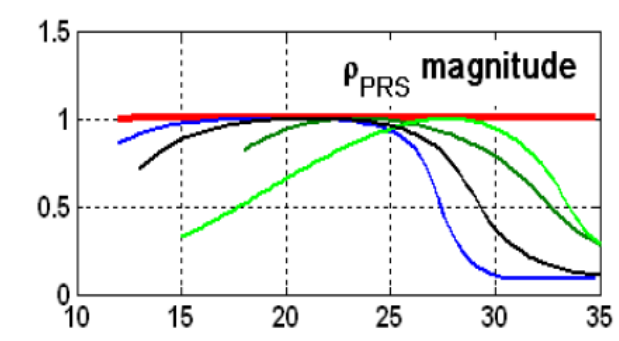

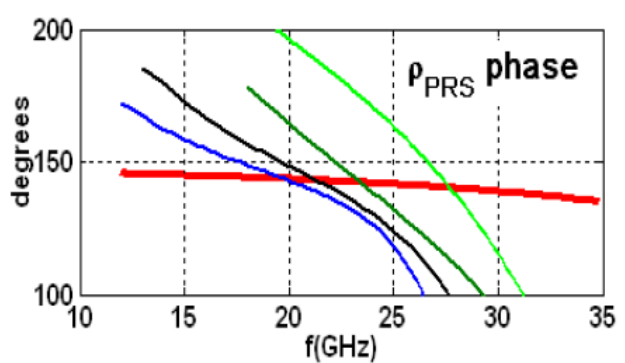

(a)

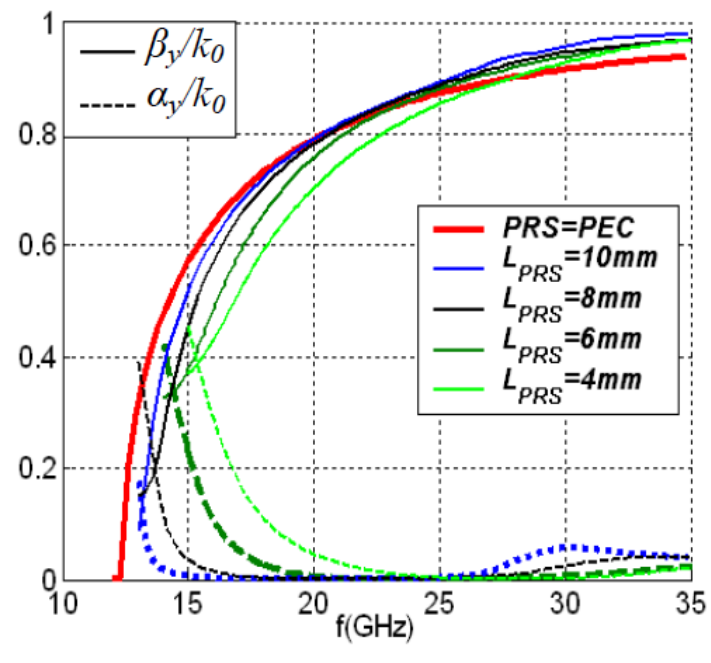

(b)

Fig. 2.3.7 (a) Reflection coefficient (magnitude and phase) of a dipole-based PRS for different values of the dipoles length $\left(L_{P R S}\right)$. (b) Dispersion diagram of the PRS-HIS FP LWA (Fig. 3.2.5 in [García-Vigueras 2012-II]).

Therefore, it can be concluded that the PRS dipoles strongly affect the leakage rate of the modes in the proposed LWA. Thus, this feature can be applied to the control of the radiation rate of the LWA for a given design frequency. At a fixed frequency and according to the bouncing ray model for waveguide propagation ([Pozar 2005]), the PRS reflectivity experienced by the incident wave ( $\rho_{P R S}$ in Fig. 2.3.4(b)) is a function of the length of its resonant dipoles $\left(L_{P R S}\right)$ ([Feresidis 2001]), as shown in Fig. 2.3.8(a) where the frequency is chosen as $15 \mathrm{GHz}$. The leaky-mode dispersion curves with $L_{P R S}$ at $15 \mathrm{GHz}$ are shown in Fig. 2.3.8(b). As can be seen in Fig. 2.3.8, the PRS dipoles resonate when $L_{P R S}=11 \mathrm{~mm}$, being approximately half a wavelength of the fixed operating frequency of $15 \mathrm{GHz}$. At this length, the PRS behaves as a totally reflective sheet $\left(\rho_{P R S}=1 \mid\right)$, a (conventional) FSS resonance occurs ([Goussetis 2006-I]) and the leakage rate vanishes $\left(\alpha_{y} / k_{0}=0\right)$. When $L_{P R S}$ is decreased from $11 \mathrm{~mm}$, the PRS becomes more transparent to the incident wave, thus leading to a progressive increase of the radiation rate. 
It can be concluded that the PRS dipoles strongly affect the leakage rate of the modes in the proposed LWA. Thus, this feature can be applied to the control of the radiation rate of the LWA for a given design frequency.

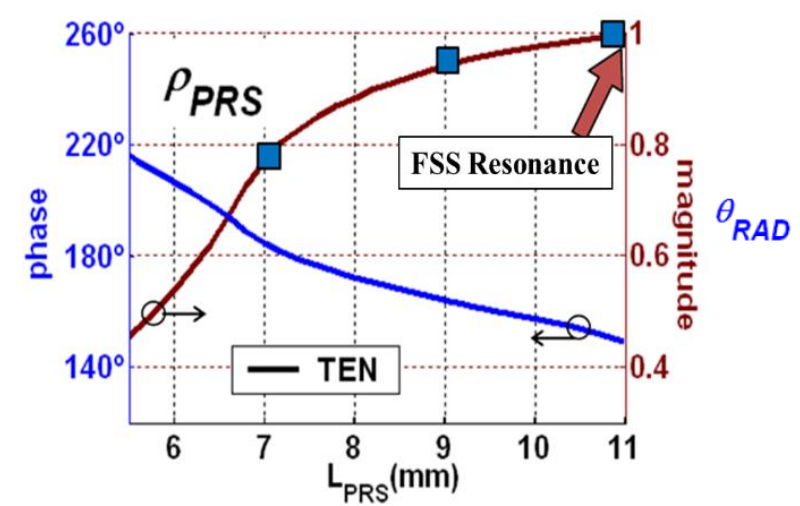

(a)

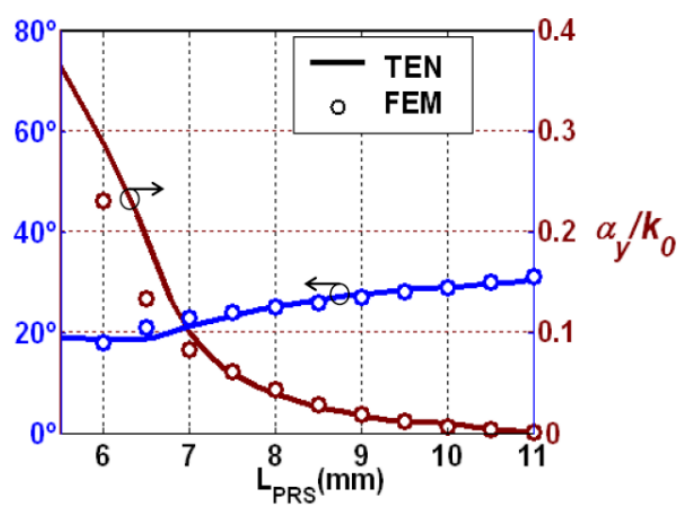

(b)

Fig. 2.3.8 (a) Reflection coefficient of the PRS and (b) Dispersion diagram of the TE leaky mode as a function of $L_{P R S}$ at fixed frequency of operation of $15 \mathrm{GHz}$ (Fig. 3.2.6 in [GarcíaVigueras 2012-II]).

At a fixed frequency and according to the bouncing ray model for waveguide propagation ([Pozar 2005]), the PRS reflectivity experienced by the incident wave ( $\rho_{P R S}$ in Fig. 2.3.4(b)) is a function of the length of its resonant dipoles ( $\left.L_{P R S}\right)$ ([Feresidis 2001]), as shown in Fig. 2.3.8(a) where the frequency is chosen as $15 \mathrm{GHz}$. The leaky-mode dispersion curves with $L_{P R S}$ at 15 $\mathrm{GHz}$ are shown in Fig. 2.3.8(b). Also, it can be seen in Fig. 2.3.8(a) that the PRS dipoles resonate when $L_{P R S}=11 \mathrm{~mm}$, being approximately half a wavelength of the fixed operating frequency of $15 \mathrm{GHz}$. At this length, the PRS resonate ([Goussetis 2006-I]), behaving as a totally reflective sheet $\left(\rho_{P R S}=1 \mid\right)$, and hence the leakage rate vanishes $\left(\alpha_{3} / k_{0}=0\right)$. When $L_{P R S}$ is decreased from $11 \mathrm{~mm}$, the PRS becomes more transparent to the incident wave, thus leading to a progressive increase of the radiation rate.

This phenomenon is also illustrated in Fig. 2.3.9, where the leaky-mode electric field inside the LWA is plotted for different values of $L_{P R S}$. It is seen that as $L_{P R S}$ is reduced from $11 \mathrm{~mm}$, more energy illuminates the top aperture of the antenna, thus increasing the leakage level. Consequently, the possibility of controlling the radiation rate by varying the PRS dipoles length is verified. Yet, when $L_{P R S}$ is varied, a second order effect occurs: the pointing angle $\theta_{R A D}$ is also altered to some extent as shown in Fig. 2.3.8(b). This deviation is due to the dependence of $\theta_{R A D}$ on the phase of $\rho_{P R S}$, which also varies with $L_{P R S}$ as illustrated in Fig. 2.3.8(a). This issue is addressed in the next subsection. 


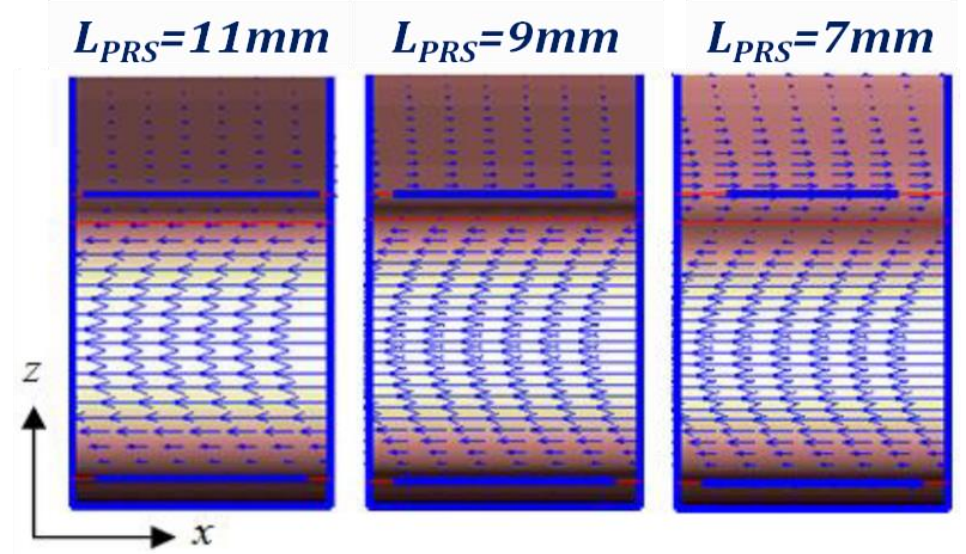

(a)

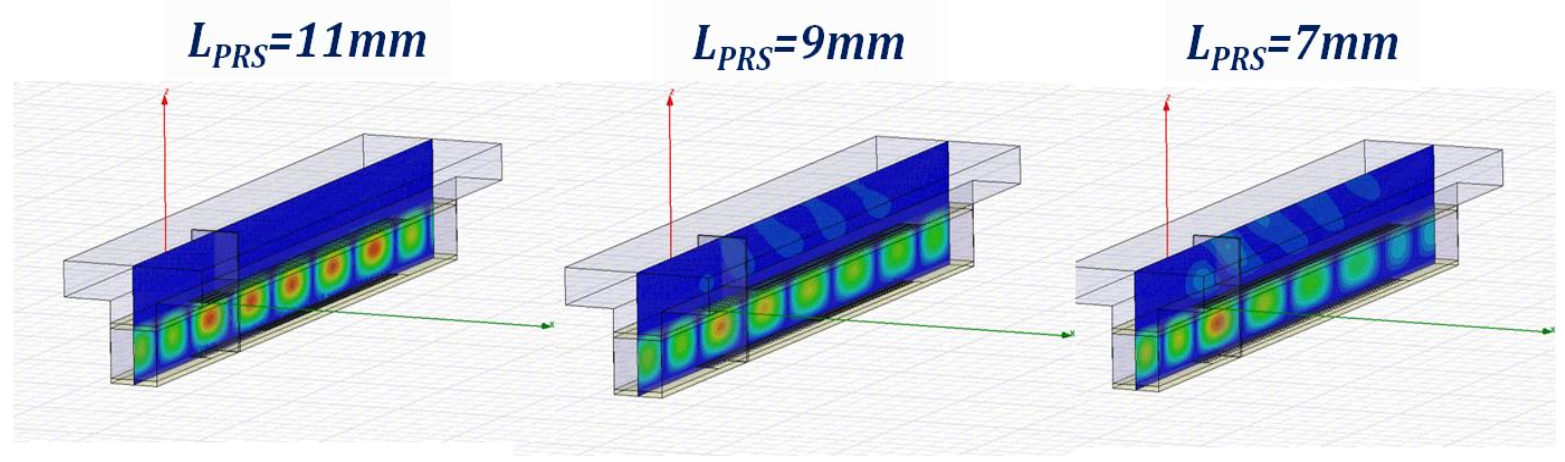

(b)

Fig. 2.3.9 (a) Transversal and (b) longitudinal electric field of the TE leaky mode for different configurations of $L_{P R S}(15 \mathrm{GHz})$ (Fig. 3.2.7 in [García-Vigueras 2012-II]). 


\subsubsection{Effect of the HIS}

As previously mentioned, the bottom of the antenna is loaded with a HIS, which consists of a dipole-based FSS on a grounded dielectric substrate. One of the first studies about these electromagnetic surfaces can be found in [Sievenpiper 1999], where it is explained that a HIS presents the interesting feature of exhibiting a variable input impedance. This is equivalent to say that the waves that impinge on a HIS can experience a variable reflection phase. In particular, this impedance varies with frequency, the angle of incidence and also with the geometry of the scatterers ([García-Vigueras 2012-II]).

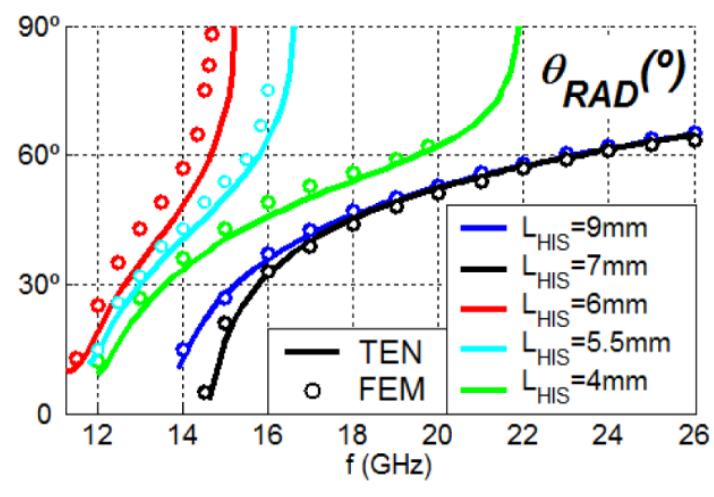

(a)

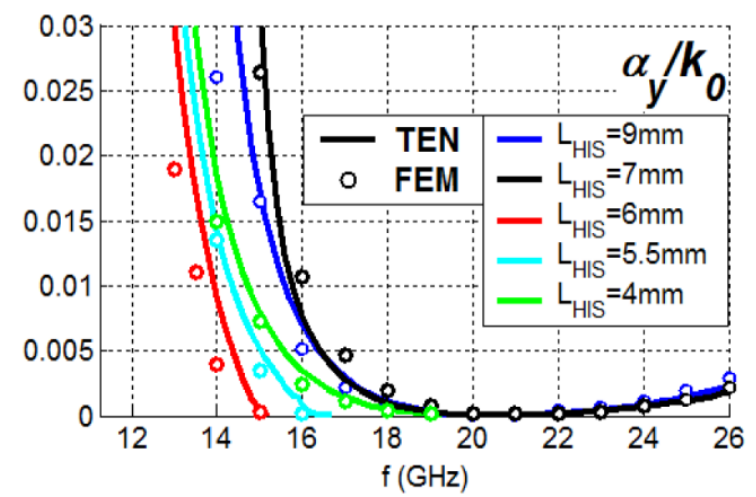

(b)

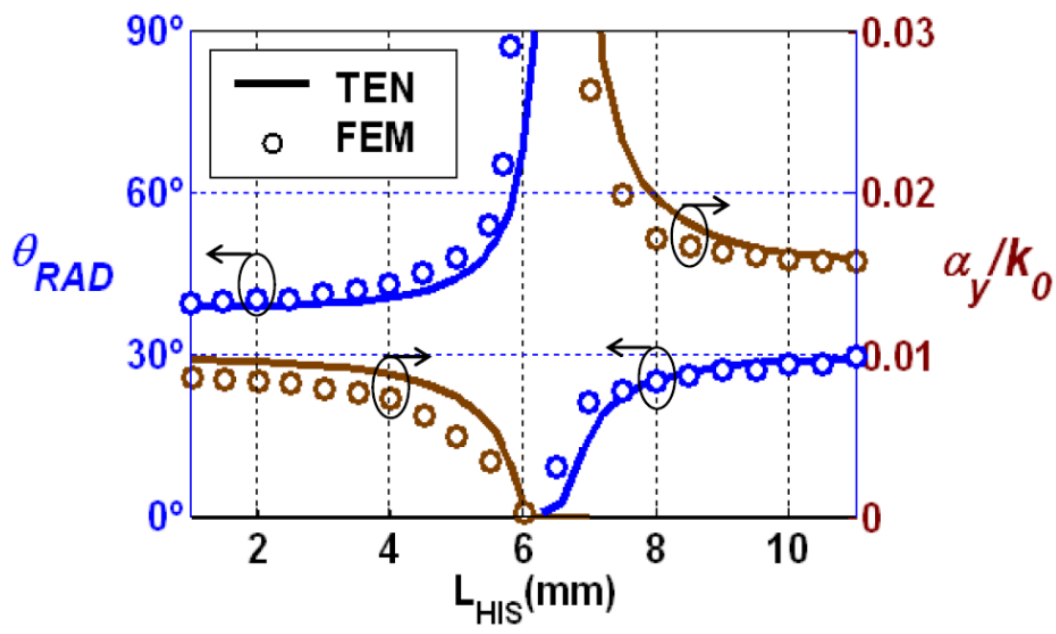

(c)

Fig. 2.3.10 Leaky-mode dispersion curves of the 1D FP LWA in Fig. 2.3.4(a) for different lengths of the HIS dipoles $\left(L_{H I S}\right)$. (a), (b) Dispersion with frequency, (c) Dispersion with $L_{H I S}$ at 15GHz (Fig. 3.2.8 in [García-Vigueras 2012-II]).

In particular, it is said that the HIS is resonating when it presents a reflection phase of zero degrees, behaving as an Artificial Magnetic Conductor (AMC). This artificial surfaces are often employed for miniaturizing waveguides, due to the fact that they can modify the effective height of the cavity ([Feresidis 2005], [Goussetis 2006-II]). In particular, in the 1D LWA under study, 
the HIS modifies the effective height of the resonant FP cavity formed by the two PCBs and therefore, it strongly affects the frequency dispersion curves of the $\mathrm{TE}_{01}$ leaky-mode, thus providing the control over the pointing angle at a fixed frequency. In a bouncing ray model for waveguide propagation, the length of the HIS dipoles $\left(L_{H I S}\right)$, determines the reflection phase experienced by the waves propagating inside the waveguide ([Feresidis 2005]). Fig. 2.3.10(a) and (b) show respectively how $L_{H I S}$ affects the LWA pointing angle and radiation rate, shifting the cutoff frequency from $11 \mathrm{GHz}$ to $15 \mathrm{GHz}$. For a fixed frequency, the pointing angle is increased and the leakage rate is reduced when the cutoff frequency is decreased (higher effective cavities) ([Oliner 2007]). Fig. 2.3.10(c) shows the dispersion curves with $L_{H I S}$ at the operation frequency of $15 \mathrm{GHz}$. In this figure it is clear that by changing $L_{H I S}$ it is possible to scan the pointing angle in a wide range. At this point it is worth noting an interest effect that the HIS causes in the dispersion of the leaky modes that propagate along the cavity.

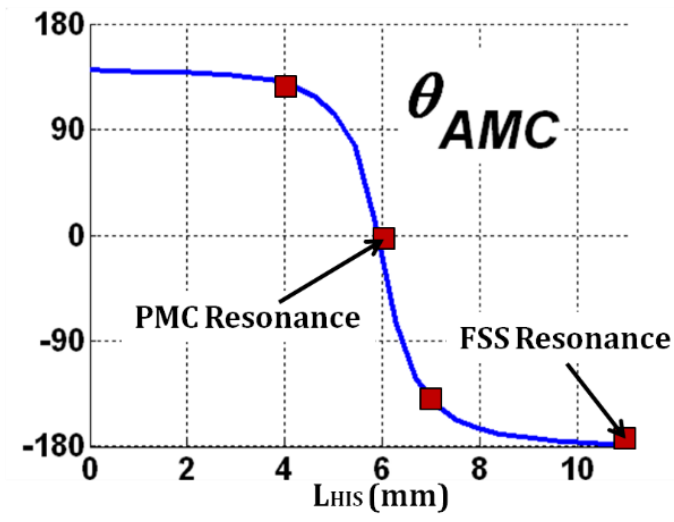

(a)
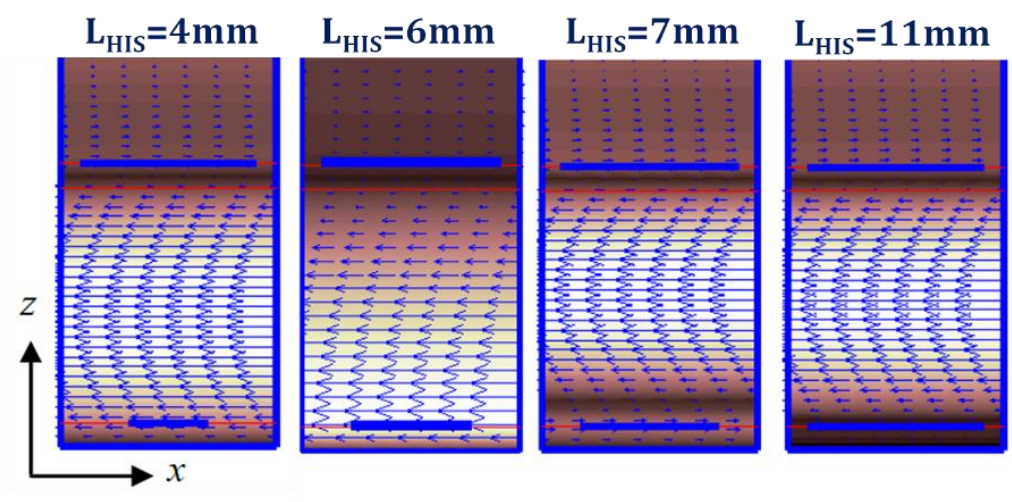

(b)

Fig. 2.3.11 (a) Reflection coefficient of the dipole-based HIS as a function of $L_{H I S}$ and (b) Electric field pattern in the transversal section of the FP LWA in Fig. 2.3.4(a) obtained from the TEN at 15GHz (Fig. 3.2.9 in [García-Vigueras 2012-II]).

As it was explained in Section 2.2.1, leaky modes in empty rectangular waveguides do not reach the surface wave regime $\left(\beta_{y} / k_{0}>1\right)$. However, as aforementioned in the introduction, waveguides can be loaded with engineered periodic surfaces to produce unusual dispersion characteristics 
(e.g. [Caiazzo 2004], [Goussetis 2007-I, II], [Seager 1999], [Yang 1999]...). In the case of the 1D LWA under study, the HIS allows the leaky modes reach the surface wave regime inside the empty waveguide, as it can be seen in Fig. 2.3.10(a).In order to understand this effect, Fig. 2.3.11(a) shows the variation of the reflection phase seen by an incoming plane-wave at the HIS interface ( $\theta_{H I S}$ in Fig. 2.3.4(a)) with $L_{H I S}$ at $15 \mathrm{GHz}$, while Fig. 2.3.11(b) illustrates the near field patterns inside the LWA. When $L_{H I S}=11 \mathrm{~mm}$ (approximately half a wavelength at $15 \mathrm{GHz}$ ), a FSS resonance occurs and the HIS behaves as a Perfect Electric Conductor (PEC) $\left(\theta_{H I S}=-180^{\circ}\right.$ in Fig. 2.3.11(a)), providing a given pointing angle of $\theta_{R A D}=30^{\circ}$ (see Fig. 2.3.10(b)).

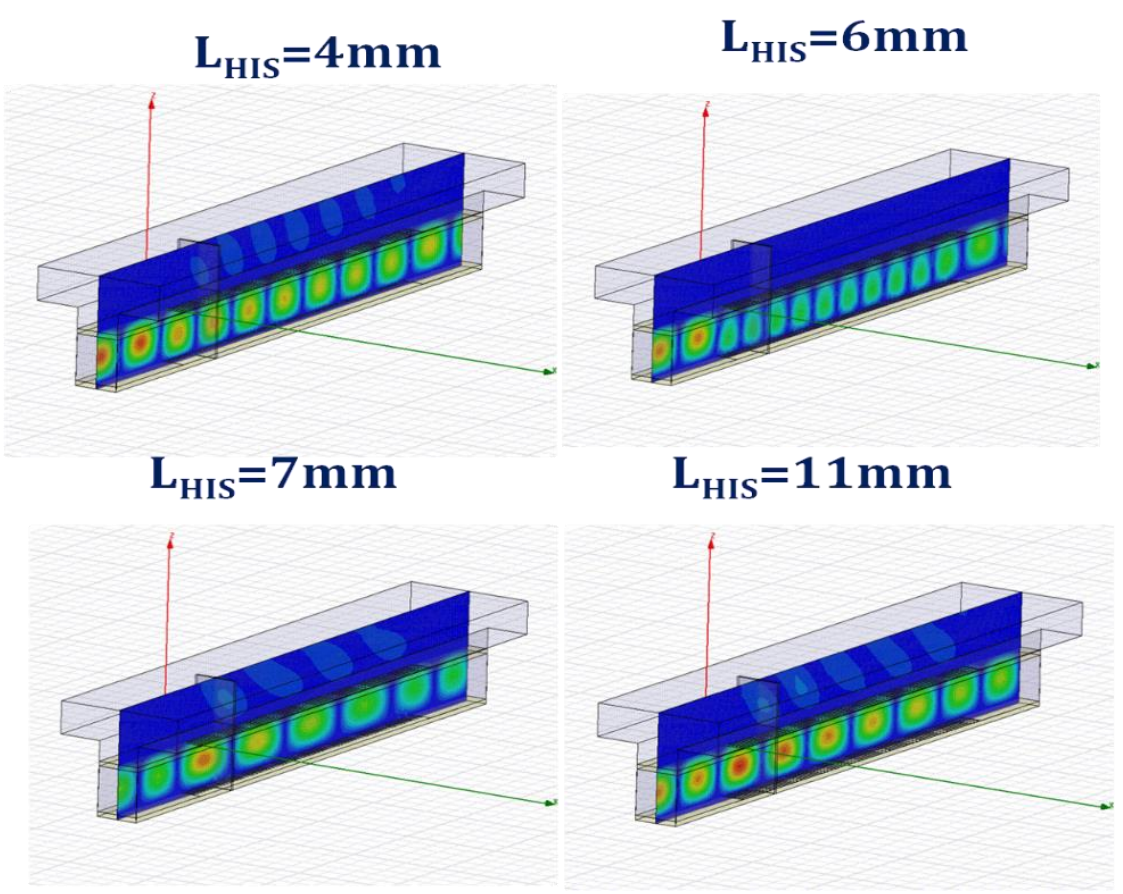

Fig. 2.3.12 Longitudinal electric field of the leaky modes for different configurations of $L_{H I S}$ at $15 \mathrm{GHz}$ (Fig. 3.2.10 in [García-Vigueras 2012-II]).

As observed, $\theta_{H I S}$ increases as $L_{H I S}$ is reduced, producing a smaller effective resonant cavity (see $L_{H I S}=7 \mathrm{~mm}$ in Fig. 2.3.11(b)), and reducing $\theta_{R A D}$ close to broadside $\left(\theta_{R A D}=0^{\circ}\right.$ for $L_{H I S}=7 \mathrm{~mm}$ in Fig. 2.3.10(c)). Maximum pointing angle at endfire $\left(\theta_{R A D}=90^{\circ}\right)$ is reached in this case for $L_{H I S}=6.1 \mathrm{~mm}$ in Fig. 2.3 .10 (c). When $L_{H I S}=6 \mathrm{~mm}$, the HIS provides a Perfect Magnetic Conductor (PMC) resonance $\left(\theta_{H I S}=0^{\circ}\right.$ in Fig. 2.3.11(a)). In this case, the electric field is maximum at the HIS interface (see $L_{H I S}=6 \mathrm{~mm}$ in Fig. 2.3.11(b)), producing an effective cavity of double height and pointing angle of $\theta_{R A D}=70^{\circ}$. Further decrease of $L_{H I S}$ continues increasing $\theta_{H I S}$ and reduces both the effective cavity height and the correspondent pointing angle $\theta_{R A D}$ (see $L_{H I S}=6 \mathrm{~mm}$ and $4 \mathrm{~mm}$ in Fig. 2.3.10(c) and Fig. 2.3.11(b)).

The effect of $L_{H I S}$ on $\beta_{y}$ can be equivalently observed in Fig. 2.3.12, where it is plotted the field distribution inside the FP cavity in the longitudinal plane of the LWA. The distance between 
two consecutive field nulls in the $y$ axis corresponds to $\lambda_{y} / 2$, which is related to the longitudinal wavenumber of the leaky wave: $\beta_{y}=2 \pi / \lambda_{y}$. Therefore, taking into account the relation between $\theta_{R A D}$ and $\beta_{y}$ (Eq.(2.2.4)), the evolution of $\beta_{y}$ with $L_{H I S}$ observed in Fig. 2.3.12 is coherent with the previous discussion. Therefore, one can conclude that the pointing angle of the proposed LWA can be tuned by changing the length of the dipoles in the HIS.

The modification of the pointing angle also involves the inherent inverse variation in the leakymode radiation rate shown in Fig. 2.3.10. It is well known that as the pointing angle of a leakywave is increased, the associated leakage rate decreases ([Oliner 2007]). This fact can be easily understood from a ray optics point of view, as illustrated in the picture of Fig. 2.3.13, where it is shown how higher radiation rates are naturally associated to lower radiation angles because the leaky wave reaches the top radiating surface more times per unit length.
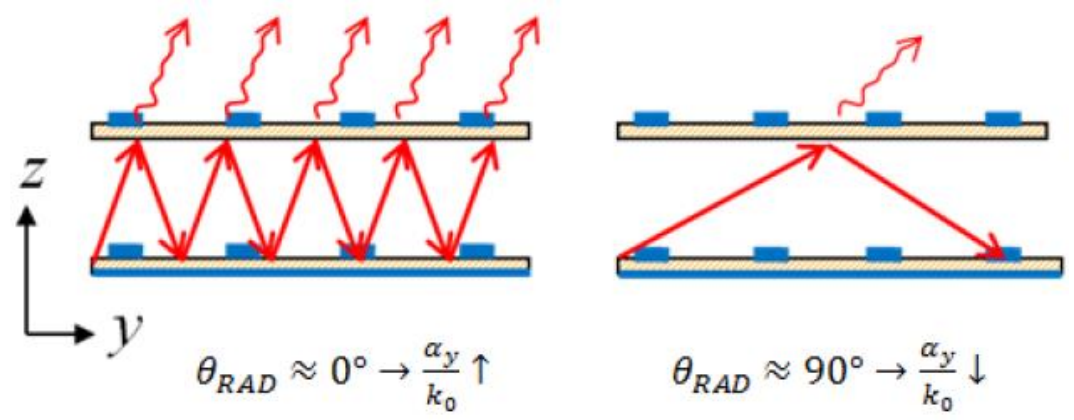

Fig. 2.3.13 General relation between the pointing angle and radiation rate of a leaky-wave (Fig. 3.2.11 in [García-Vigueras 2012-II]). 


\subsubsection{Control of the pointing angle and beamwidth in $1 D$ FP LWAs}

With the observations derived from previous sections, it has been demonstrated that the phase constant and the leakage rate of the 1D FP LWA can be flexibly controlled by the lengths of the printed dipoles of the PRS and the HIS respectively. From the design equations derived in Section 2.2.1 (Eq.(2.2.4)-Eq.(2.2.10)), it is clear that the phase constant will be the primary factor to control the radiation angle $\left(\theta_{R A D}\right)$, whereas the leakage factor control the beamwidth $(\Delta \theta)$ (and/or the radiation efficiency) of the LWA. From these concepts, and with the proper synthesis of the dipole lengths of the PRS and HIS, different LWAs with different pointing angle and beamwidth can be designed. To this aim, [García-Vigueras 2012-II] proposed a synthesis tool based on the TRM, which looks for the dimensions of the structure ( $L_{P R S}$ and $L_{H I S}$ ) from the specified pointing angle, beamwidth and radiation efficiency. This method has been key for the design of more complex tapered 1D and 2D FP LWAs with enhanced aperture efficiency, directivity, or reduction of side lobe levels (e.g. implementing different illuminations such as uniform or cosine-type) ([García-Vigueras 2011-I, II, 2012-II]). Fig. 2.3.14 shows the 2D contour curve plots which relate $L_{P R S}$ and $L_{H I S}$ with $\theta_{R A D}$ and $\alpha$. From these curves, it is worth to note that both parameters are not totally independent, but they are coupled, as was previously observed. Thus, $L_{P R S}$ perturbs (smoothly) the radiation angle of the antenna and strongly the leakage rate, whilst $L_{H I S}$ also affects the leakage rate as affect strongly the radiation angle, as commented before (Fig. 2.3.13).

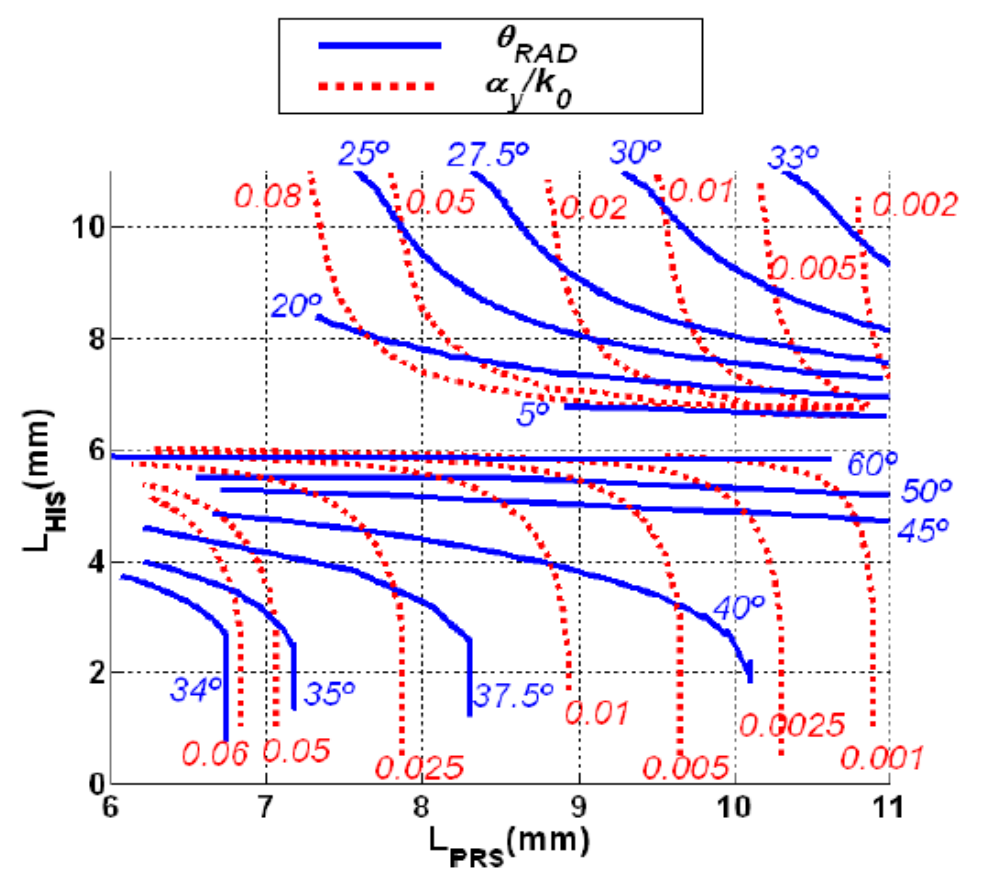

Fig. 2.3.14 2D contour curve plots of $\theta_{R A D}$ and $\alpha / k_{0}$ obtained at $15 \mathrm{GHz}$ (Fig. 3.3.1 in [GarcíaVigueras 2012-II]). 

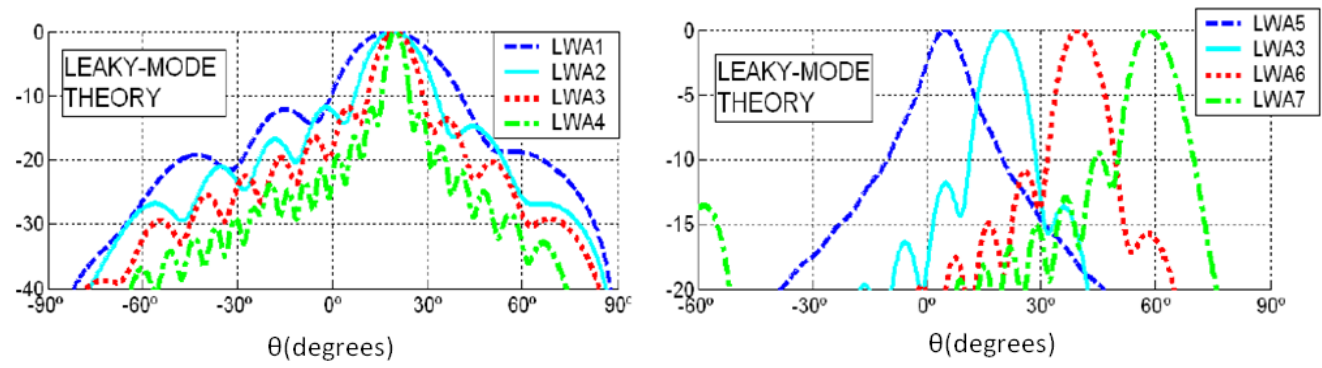

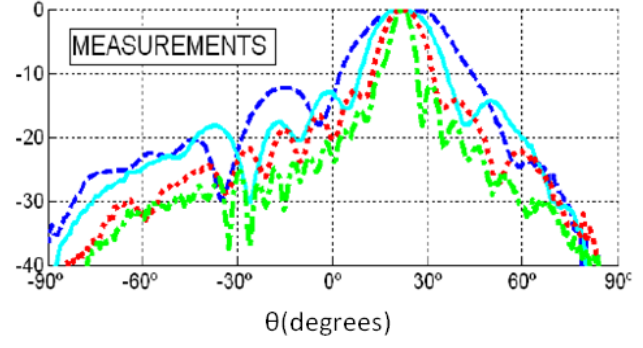

(a)

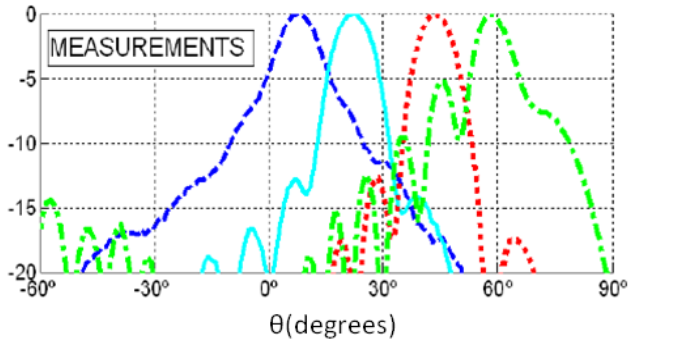

(b)

Fig. 2.3.15 Theoretical and measured normalized radiation patterns for the LWAs in Table 2.3.1 (a) showing the control of the beamwidth $(\Delta \theta)$ and (b) showing the control of the pointing angle $\left(\theta_{R A D}\right)$ (Fig. 3.3.4 and Fig.3.3.5 in [García-Vigueras 2012-II]).

\begin{tabular}{lc} 
LWA 5 & $5^{\circ}$ \\
LWA 6 & $40^{\circ}$ \\
LWA 7 & $60^{\circ}$ \\
\hline
\end{tabular}

Table 2.3.1 Electrical and geometrical parameters of the LWAs designed at $15 \mathrm{GHz}$ (Table.

3.3.1 in [García-Vigueras 2012-II]).

From these design curves, the method finds the proper solutions to obtain the desired radiation pattern. Seven different uniform (non-tapered) designs with different radiation angle and beamwidth (controlled by proper leakage rate) were firstly presented in this work. The simulated and measured radiation patterns obtained are presented in Fig. 2.3.15. These designs were the first prototypes which were designed and fabricated in [García-Vigueras 2012-II] to demonstrate the capacity to flexibly control the principal radiation characteristics of these antennas. It is clear how the beamwidth can be changed, as a constant pointing angle is kept, as observed in the radiation patterns shown in Fig. 2.3.15(a) for cases LWA1, 2, 3 and 4. On the other hand, patterns presented in Fig. 2.3.15 for cases LWA3, 5, 6 and 7 show four passive 1D FP LWAs with similar beamwidth, but pointing at different directions. All the prototypes were 
designed to keep a radiation efficiency of $\eta_{R A D}=90 \%$. The value of the dipole lengths as the numerical values of beamwidth and pointing angle can be found in Table 2.3.1. 


\subsection{Electronic-Steering 1D FP LWA}

\subsubsection{General Structure}

The structure of the electronic-steering 1D FP LWA proposed in this chapter and its main geometrical parameters are shown in Fig. 2.4.1(a). As observed, this structure is inspired by the structure of the passive double-layer 1D FP LWA presented in [García-Vigueras 2012-II], reviewed in the previous section. As in the passive LWA, this reconfigurable version is also based on a parallel-plate waveguide (PPW) loaded with two printed-circuit boards (PCBs): a top PRS and a bottom HIS (Fig. 2.4.1(b)). Both PCBs are separated at a distance $H$, comprising the one-dimensional FP resonant cavity, which will be also excited by a $\mathrm{TE}_{01}$ rectangular waveguide mode, in order to operate with the TE leaky mode.

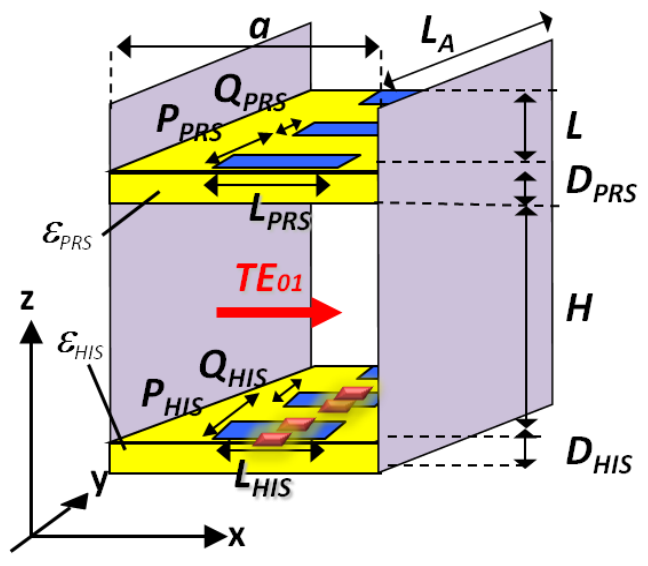

(a)

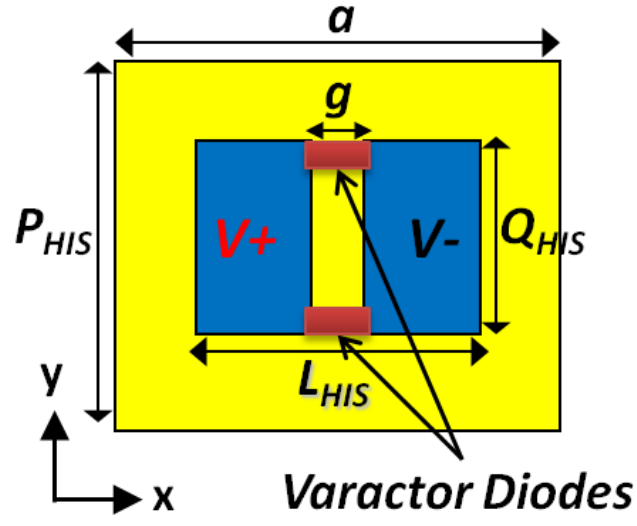

(b)

Fig. 2.4.1 a) Scheme of the proposed reconfigurable 1D FP LWA b) Detailed scheme of the tunable high impedance surface unit cell ( $a=25 \mathrm{~mm}, H=25.2 \mathrm{~mm}, L=13.6 \mathrm{~mm}, D_{H I S}=1.524 \mathrm{~mm}$, $\varepsilon_{r H I S}=3, P_{H I S}=30 \mathrm{~mm}, Q_{H I S}=14 \mathrm{~mm}, L_{H I S}=17 \mathrm{~mm}, g=1 \mathrm{~mm}, D_{P R S}=0.8 \mathrm{~mm}, \varepsilon_{r P R S}=4.4, P_{P R S}=20 \mathrm{~mm}$,

$$
\left.Q_{P R S}=18 \mathrm{~mm}, L_{P R S}=22 \mathrm{~mm}\right) \text {. }
$$

Remembering the results reported by [García-Vigueras 2012-II], reviewed in Subsections 2.3.3 and 2.3.4, it can be thought that the leakage rate $(\alpha)$ of the TE leaky mode can be mainly adjusted by properly designing the resonant length of the top PRS patches ( $L_{P R S}$ in Fig. 2.4.1(a)), whilst the resonant length of the HIS patches ( $L_{H I S}$ in Fig. 2.4.1(a)) controls the phase constant $(\beta)$ of the leaky mode (and hence the pointing angle $\theta_{R A D}$ of the antenna, as demonstrated by Eq.(2.2.4)). However, in this case, a reconfigurable version of the double-layer (PRS-HIS) 1D FP LWA is envisaged by replacing the passive HIS by a tunable HIS loaded with varactors (see the scheme of a single unit cell of the HIS in Fig. 2.4.1(a)). This tunable HIS is able to change electronically its reflection phase ([Chicherin 2006, 2011-I, II], [Hum 2005, 2007], [Mias 2007], 
[Sterner 2009] [Weily 2008]), allowing an electronic reconfiguration of the phase constant of the leaky mode, which is translated into electronic steerability of the beam. This simple hypothesis comes from the observations performed when the resonant length $L_{H I S}$ of the HIS dipoles were altered (review in Subsection 2.3.4). Then, it was probed that the HIS reflection phase $\left(\theta_{H I S}\right.$ in Fig. 2.3.4(b)) and hence the resonance, and the overall dispersion properties of the FP cavity were altered with this parameter. The point is that if it is desired an electronic control of the pointing angle for real-time applications, the HIS must be able to tune by some flexible mechanism its reflection phase. As will be explained in Subsection 2.5.4, the tunable HIS here employed will be able to change electronically its reflection phase ([Chicherin 2006, 2011-I, II], [Hum 2005, 2007], [Mias 2007], [Weily 2008]), by tuning the varactors, what will be equivalent to change the physical length of the patches in the resonant direction, allowing an electronic control of the phase constant of the leaky mode in the FP cavity, and ultimately the electronic steerability of the beam, as will be demonstrated.

The reconfigurable antenna presented has been designed to operate in C-band, particularly around $5.6 \mathrm{GHz}$. At higher frequencies, varactors have higher losses, and are less operative because of their low dynamic range. Other alternatives, such as RF MEMS are usually more expensive than varactors, and today are still an immature technology which presents other types of issues for continuous reconfiguration ([Yashchyshyn 2010-II]). However, this technology could be taken into account to scale this antenna to operate at higher frequency bands (e.g. Xband or mm-wave range), where they are being usually employed, as shown in recent contributions ([Devogobic 2010, 2014-I, II], [Chicherin 2011-I, II], [Raisanen 2012]...).

Regarding the FSSs which constitute the PRS and HIS, notice that they have been implemented by periodic arrays of resonant patches (instead of resonant dipoles). The reasons are several. Patches are usually less resonant (lower Q factor) compared to dipoles, and hence also present lower losses ([Costa 2012], [Sievenpiper 2003]), what it is important, especially for the HIS, as will be shown in Section 2.8. In addition, structural reasons have led us to this type of configuration, such as to provide enough space to incorporate the varactor diodes in the HIS. In fact, as will be explained in next subsections, the tunable HIS geometry employed here has been highly tested in previous contributions which have worked with the same or similar tunable surfaces ([Hum 2007], [Sievenpiper 2003, 2005], [Weily 2008]). 


\subsubsection{Analysis and Design Methodology}

In general, the analysis and design process employed for the reconfigurable antennas is more complex than for passive antennas ([Rutschlin 2013]). Reconfigurable structures employ diverse mechanisms to allow the electronic control of their dynamic response. These antennas usually operate in different modes which alter the input impedance of the device, requiring of more complex or especially well-optimized feeding sources to match the antenna at any operating mode. Sometimes, reconfigurable sources are considered to this aim, what complicates even more the design process (e.g. [Debogovic 2010]). In addition, bias networks are required to properly control the tunable elements, what is more or less complex, depending on the structure geometry, the number of control signals and the type of tunable devices employed. Concerning the simulation of these structures, tunable elements such as diodes, MEMS or tunable materials employed for many reconfigurable devices, require of accurate models which in many occasions are not provided by the manufacturers, and require of an additional extracting process to integrate these components inside the full-wave simulation tools in the final design process of the structure. These simulators are often commercial software tools (e.g. [HFSS 2011], [CST 2012]) intended to analyze non-specific electromagnetic problems and, although they can provide quite accurate prediction (as long as the proper models of the tunable devices are integrated) they require of high computational resources and time. This issue has been one of the main drags which has slowed down the development of new structures in the reconfigurable and general microwave field, and has made that RF engineers create their own home-made specific tools to analyze and design complex structures in an efficient and accurate way. The implementation of efficient techniques for aiding the analysis and design process of complex structures, such as electrically large and/or complex reconfigurable antennas, is always of great interest for practical designs in order to avoid blind optimization procedures by intense campaigns of full-wave simulations, that otherwise would be necessary in the final model simulations and which would be unfeasible in terms of computational cost and time. In this thesis, an efficient methodology have been followed, based on different techniques for the design of FSS and the dispersion analysis of the FP LWAs proposed, which will serve as a quick guideline for the study of the initial dimensions of the structures, prior to a finer optimization of the antenna by full-wave methods. It can be divided in five steps:

\section{Analysis and design of the periodic surfaces which compose the PRS and the HIS}

From the designs proposed in recent works ([Hum 2007], [Weily 2008]), an initial PRS and HIS designs have been adopted and re-designed for our purposes. In particular, a full-wave incident plane-wave analysis have been performed by commercial tools for the study of the reflection 
properties of the PRS and the tunable HIS, to obtain the desired response at the frequency of interest $(5.6 \mathrm{GHz}$ in this case).

\section{Characterization by a TEN}

In this step, a Transverse Equivalent Network (TEN) has been developed to characterize the FP LWA by equivalent transmission lines and admittances/impedances which model each element of the structure (the FSSs of the PRS and HIS, and the radiation produced at the edge of the PPW) as a function of the design parameters of interest. The complexity to obtain this equivalent network is to characterize with simple, but accurate expressions the equivalent admittances of the FSS surfaces of the PRS and HIS. As will be explained in next subsections, two methods have been employed to obtain quasi-analytical expressions of the equivalent admittances used to model the response of the periodic surfaces of the PRS and HIS: the polezero method proposed in [Maci 2005], and the modified pole-zero method presented in [GarcíaVigueras 2010]. On one hand, the original pole-zero method of [Maci 2005] can be employed to characterize the patch-based FSS of the HIS and the FSS of the PRS as a function of frequency. On the other hand, with the modified method it is aimed the characterization of the PRS admittance as a function of the physical length of the patches $\left(L_{P R S}\right)$ at the fixed design frequency of interest $(5.6 \mathrm{GHz})$. Also, for the characterization of the tunable HIS as a function of the junction capacitance of the varactor diodes $\left(C_{j}\right)$, which is essential for the analysis of the electronic scanning of the antenna, this modified pole-zero method has been re-adapted successfully to extend its applicability to characterize tunable FSS loaded with tuning lumped elements (in this case varactors).

\section{Dispersion Analysis by the Transverse Resonance Method}

Once the TEN has been obtained, the leaky-mode propagation constant $\left(k_{y}=\beta_{y}-j \alpha_{y}\right)$ can be calculated. To this aim, the Transverse Resonance Method (TRM) is employed. The TRM is a simple and powerful tool widely used in the design of LWAs ([García-Vigueras 2012-II], [Oliner 2007], [Zhao 2005-II, III]). This technique allows to perform efficiently the dispersion analysis of the leaky mode as a function of different parameters of interest. In this case, three different dispersion studies will be performed for different parameters: frequency, height of the FP cavity $(H)$, length of the PRS patches $\left(L_{P R S}\right)$ and junction capacitance $\left(C_{j}\right)$ of the varactors. As commented in Section 2.3, this method was also employed in [García-Vigueras 2012-II] for the synthesis of the dipole lengths of the FSS, in order to synthesize the different uniform and tapered passive LWAs, analyzing the TEN shown in Fig. 2.3.4(b). 


\section{Real Excitation and Final Design}

Once the dimensions of the structure have been obtained by the TRM, the final structure design is analyzed by a 3D commercial full-wave simulator. Unlike the TRM, which assume a dispersion analysis in an infinite guiding structure, the 3D model simulated is a finite antenna, with an aperture length $L_{A}$, and incorporate a real excitation of the structure. Regarding the excitation source, in order to save computational time, an optimization process of the excitation structure is performed by full-wave methods, employing a short version of the LWA in order to analyze (approximately) the mismatching. Finally, the full model with length $L_{A}$ is simulated to validate its performance.

\section{Fabrication of the Prototype and Experimental Tests}

With the final dimensions of the structure, the prototype is fabricated and tested, measuring different properties of the antenna such as the scattering parameters and the radiation patterns for different frequency ranges and operating points of the tunable HIS. 


\subsection{Characterization}

The analysis and characterization of FSSs has been widely studied for several decades ([Munk 2000], [Tretyakov 2003-I]). Depending on the application, the analysis of a FSS may be done for different purposes. On one hand, a "study of reflection" is performed when the emphasis is on the reflection properties of the FSS, as occurs for the design of new surfaces with novel reflection characteristics (e.g. [Sievenpiper 1999], [Goussetis 2006-I], [Doumanis 2012], etc.). On the other hand, the design of novel structures conceived from combining guides with embedded FSSs ([García-Vigueras 2011-II], [Goussetis 2006-II], [Feresidis 2005], etc.), as the FP LWAs studied in this work, may require of a "study of dispersion" of the structure which also needs of the analysis of the FSS to study the dispersion response of the full guiding structure. For any of these situations, the characterization of the FSS by a simple closedexpression model is desired to, for example, predict in a fast and accurate way the conventional resonances, extraordinary transmissions or apparition of grating lobes of a reflecting surface, or to analyze analytically the dispersion equations of more complex structures, to obtain its dispersion diagram. This latter problem is studied in this work, where the propagation constant of the leaky mode in the 1D FP LWA is unknown, and must be found as a function of different design parameters of interest in order to optimize the antenna scanning response (defined as the range of angles at which the antenna is able to point). To this aim, the Transverse Resonance Method (TRM) will be employed to find the leaky-mode propagation constant for a given frequency and geometry configuration. This method needs of a Transverse Equivalent Network (TEN), which characterizes the transversal section of the FP LWA. Thus, the PRS and the HIS periodic surfaces are modelled by equivalent admittances in this circuit model. These admittances are functions dependent on the parameters of interest for the design process, and will be obtained by the pole-zero expansion methods presented in [Maci 2005] and [GarcíaVigueras 2010]. A Transverse Resonance Equation (TRE) is derived from the circuit analysis to obtain the dispersion equations required to obtain the propagation constant of the leaky mode by numerical methods.

In Section 2.5.1, it is described the TEN employed to characterize the reconfigurable 1D FP LWA of Fig. 2.4.1. Then, the pole-zero methods employed to obtain the equivalent admittances of the PRS and HIS are described in Section 2.5.2. Later, the design and characterization of the PRS and the HIS are described in Sections 1.5.3 and 1.5.4. Then, the pole-zero methods will be applied for the characterization of the PRS and HIS designed. 


\subsubsection{Transverse Equivalent Network}

The TRM has been employed to obtain the complex propagation constants $\left(k_{y}=\beta_{y}-\mathrm{j} \alpha_{y}\right)$ of the leaky modes as a function of different parameters of interest. The TRM is simple and accurate but requires of a Transverse Equivalent Network (TEN) which characterizes the transversal section of the structure for the leaky mode of interest to obtain its dispersion diagram. In Fig. 2.5.1 it is shown the proposed TEN employed to analyze the dispersion of the $\mathrm{TE}_{0 \mathrm{~m}}$ leaky modes which may propagate through the structure presented in Fig. 2.4.1(a).

This equivalent circuit consists of four segments of transmission lines and three equivalent admittances. The characteristic impedance of the transmission lines are associated to a TE mode $\left(Z_{0}^{V A C}\right.$ or $Z_{0}^{\varepsilon_{r}}$, in sections where the guide is air-filled $\left(\varepsilon_{r}=1\right)$ or filled with a dielectric substrate of relative permittivity $\varepsilon_{r}$, respectively). Analyzing the circuit from the bottom, firstly a shortcircuit is found, modeling the ground plane of the HIS grounded substrate. Later, a transmission line models the HIS substrate slab with permittivity $\varepsilon_{H I S}$ and thickness $D_{H I S}$. This substrate holds the patch-based FSS with varactors employed to conceive the tunable HIS, which in this case is modeled by an equivalent admittance $Y_{H I S}\left(f, k_{y}, C_{j}\right)$. This FSS is modeled by an equivalent shunt admittance $Y_{\text {HIS. }}$ Over the HIS, a transmission line of length $H$ characterizes the air-filled FP cavity comprised between the FSS of the HIS and the dielectric substrate of the PRS, which is equivalent to another transmission line of length $D_{P R S}$ and permittivity $\varepsilon_{P R S}$. On the top of this slab, the shunt admittance $Y_{P R S}\left(f, k_{y}, L_{P R S}\right)$ models a (non-tunable) patch-based FSS employed to conceive the final PRS properties.

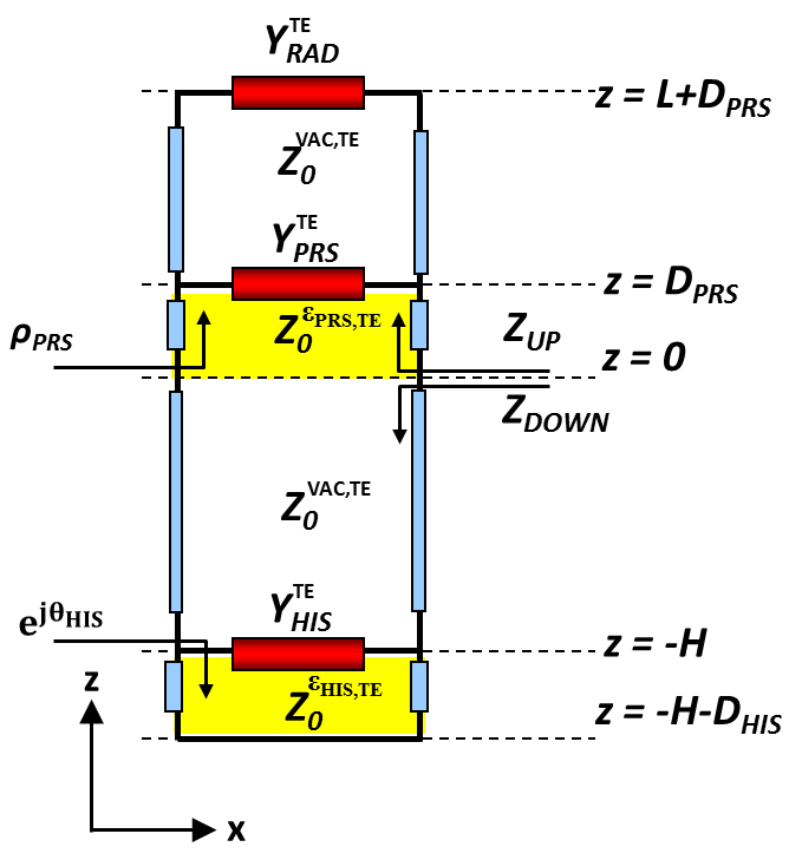

Fig. 2.5.1 Transverse Equivalent Network (TEN) of the reconfigurable 1D FP LWA. 
Finally, over the PRS, the top parallel plates of length $L$ are represented as an air-filled transmission line segment loaded with the radiation admittance $Y_{R A D}\left(f, k_{y}, a\right)$. This admittance models the radiation discontinuity encountered at the top of the open waveguide of width $a$. This equivalent radiation admittance was proposed by Nathan Marcuvitz in [Marcuvitz 1951]. It is given by a closed expression which characterizes the discontinuity observed by a plane wave which travels across an open-ended PPW with some incidence angle $\left(\theta_{i}\right)$ and impinges on an infinite aperture. It is easy to determine and has been widely employed in recent works to perform dispersion analyses in open waveguides by the TRM ([García-Vigueras 2012-II], [Gómez 2005-I]).

In order to solve $k_{y}$, the following Transverse Resonance Equation (TRE) associated to the TEN, must be satisfied:

$$
Z_{U P}\left(k_{y}, f, L_{P R S}\right)+Z_{D O W N}\left(k_{y}, f, H, C_{j}\right)=0
$$

where $Z_{U P}$ and $Z_{D O W N}$ can be obtained by simple transmission line theory. Thus, the expressions of the impedances which appear in Fig. 2.3.4 are the following ones (TE case):

$$
\begin{aligned}
& k_{z}^{V A C}=\sqrt{k_{0}^{2}-k_{y}^{2}} \\
& k_{z}^{\varepsilon_{r}}=\sqrt{k_{0}^{2} \varepsilon_{r}-k_{y}^{2}} \\
& Z_{0}^{V A C, \varepsilon_{r}}=\frac{w \mu_{0}}{k_{z}^{V A C, \varepsilon_{r}}} \\
& Z_{D O W N}^{\prime}=j Z_{0}^{\varepsilon_{H I S}} \tan \left(k_{z}^{\varepsilon_{H I S}} D_{H I S}\right) \\
& Z_{\text {DOWN }}^{\prime \prime}=\left(Y_{H I S}+1 / Z_{D O W N}^{\prime}\right)^{-1} \\
& Z_{D O W N}=Z_{0}^{V A C} \frac{Z_{D O W N}^{\prime \prime}+j Z_{0}^{V A C} \tan \left(k_{Z}^{V A C} H\right)}{Z_{0}^{V A C}+j Z_{D O W N}^{\prime \prime} \tan \left(k_{Z}^{V A C} H\right)} \\
& Z_{U P}^{\prime}=Z_{0}^{V A C} \frac{\frac{1}{Y_{R A D}}+j Z_{0}^{V A C} \tan \left(k_{Z}^{V A C} L\right)}{Z_{0}^{V A C}+j \frac{1}{Y_{R A D}} \tan \left(k_{Z}^{V A C} L\right)} \\
& Z_{U P}^{\prime \prime}=\left(Y_{P R S}+1 / Z_{U P}^{\prime}\right)^{-1} \\
& Z_{U P}=Z_{0}^{P R S} \frac{Z_{U P}^{\prime \prime}+j z_{0}^{\varepsilon_{P R S}} \tan \left(k_{z}^{\varepsilon_{P R S}} D_{P R S}\right)}{z_{0}^{\varepsilon_{P R S}}+j Z_{U P}^{\prime \prime} \tan \left(k_{z}^{\varepsilon_{P R S}} D_{P R S}\right)}
\end{aligned}
$$

As observed, the equations derived for the TRE will be a function of frequency, $H, L_{P R S}$ and $C_{j}$, which are the parameters of interest for the optimization of the design, besides the unknown 
leaky-mode propagation constant $\left(k_{y}\right)$, so that the modal solutions of the structure can be found by solving the previous TRE at a specific frequency or in a given frequency range, and for a specified geometry. All the admittances of the TEN (including the radiation admittance $Y_{R A D}$ ) are supposed dependent on the unknown $k_{y}$ since the reflection response of these surfaces is intrinsically related to the internal incidence angle $\left(\theta_{i}\right)$ with which the wave impinges on the PRS, the HIS and on the open aperture at the end of the PPW, and which is inherently related to the unknown $k_{y}$ by $\sin \theta_{i}=\operatorname{Re}\left\{k_{y}\right\} / k_{0}$. Also note that $Y_{P R S}$ and $Y_{H I S}$ will be in charge of introducing the dependences with $\left(L_{P R S}\right)$ and the varactors' junction capacitance $\left(C_{j}\right)$, which is key for the electronic control of the pointing angle, respectively. Thus, it is expected that the expressions found for $Y_{P R S}\left(f, k_{y}, L_{P R S}\right)$ and $Y_{H I S}\left(f, k_{y}, C_{j}\right)$ will account for this angle dependence $\left(k_{y}\left(\theta_{i}\right)\right)$ apart from the desired parameters of interest ([García-Vigueras 2010], [Maci 2005]) in order to predict accurately the dispersion of the structure

In next section it is appreciated that these admittances can be extracted by different methods. To this aim, in this work we have employed different Pole-Zero Methods, described in Subsection 2.5.2, to extract the equivalent admittances which model the FSS of the HIS and the PRS as a function of different parameters of interest such as frequency, $L_{P R S}$ or $C_{j}$. 


\subsubsection{Pole-Zero Method}

In the frame of this thesis, it is aimed the characterization of the FSSs which constitute the PRS and the HIS, in order to analyze a more complex type of structure such as the FP LWAs here proposed. In the literature, different analytical expressions have been derived for specific element geometries [Costa 2012], [Tretyakov 2003-I], [Goussetis 2006-I], [Luukonen 2008, 2009], [Medina 2010], [García-Vigueras 2012-I], [Mittra 1988], [Sievenpiper 1999]). Also, tunable HIS with varactors have been analyzed from an equivalent circuit viewpoint in [Hum 2007], [Luukkonen 2008], [Costa 2011]. However their application is limited to those geometries for which analytical solutions have been obtained. In this work, two methods have been applied for the characterization of the FSSs of the PRS and the HIS, depending on the type of the analysis performed. For a frequency dispersion analysis, the pole-zero method proposed by Maci [Maci 2005] will be employed. However, for a fixed-frequency parameter dispersion, the modified pole-zero method proposed in [García-Vigueras 2010], will be more convenient.

The original pole-zero method presented in [Maci 2005] employs full-wave results and Foster's theorem, to derive a quasi-analytical equivalent impedance of a planar two-dimensional periodic surface. This method stated how the reflection frequency response of an arbitrarily-shaped twodimensional FSS can be approximated by a simple polynomial $\left(Y_{F S S}\left(k_{y}, w\right)\right)$ which poles and zeros can be extracted from a few full-wave simulations. Thus, in [Maci 2005] it is proven that assuming a lossless case, $Y_{F S S}$ respects Foster's theorem and therefore, has the same analytic properties as an LC resonant circuit in frequency, i.e.: the poles and zeros are in the real axis, they are simple and alternate and symmetric w.r.t the origin $(w=0)$, where also there must be a pole or zero. The expression will be dependent on the frequency $w$, and the propagation constant of the incident wave in the longitudinal direction $k\left(k_{y}\right.$, for the case of a TE incident plane wave which impinges on the FSS with an angle $\theta_{i},\left(\phi=90^{\circ}\right)$ as depicted in Fig. 2.5.2(a)), which corresponds to the unknown variable to figure out in our dispersion analysis problem. Considering the TE incidence of scenario presented in Fig. 2.5.2(a), $Y_{F S S}\left(k_{y}, w\right)$ can be approximated by the next polynomial function:

$$
Y_{F S S}\left(k_{y}, \mathrm{w}\right)=j \frac{\mathrm{w}\left[\mathrm{w}-w_{\mathrm{z} 1}\left(k_{y}\right)\right] \ldots\left[\mathrm{w}-w_{\mathrm{zm}}\left(k_{y}\right)\right]}{\left[\mathrm{w}-w_{\mathrm{p} 1}\left(k_{y}\right)\right] \ldots\left[\mathrm{w}-w_{\mathrm{pn}}\left(k_{y}\right)\right]}
$$

being $w_{z 1}\left(k_{y}\right)<w_{p 1}\left(k_{y}\right)<w_{z 2}\left(k_{y}\right)<w_{p 2}\left(k_{y}\right) \ldots$, the frequencies where the poles and zeros of $Y_{F S S}\left(k_{y}, w\right)$ are located. In a more general case, depending on the incidence, $k_{y} / k_{x}$ are used in Eq.(2.5.11) for the TE /TM respectively. For oblique incidence, where $\phi$ is such that TE $\left(\phi=90^{\circ}\right)$ and $\mathrm{TM}\left(\phi=0^{\circ}\right)$ polarization are coupled, the admittance can be described as a matrix, changing the problem into calculating a TE and a TM admittance, following [Maci 2005]. In 
this way, the method can be extended to analyze not only infinitely thin dipole-based FSS, but also for example rectangular strips or square patches.

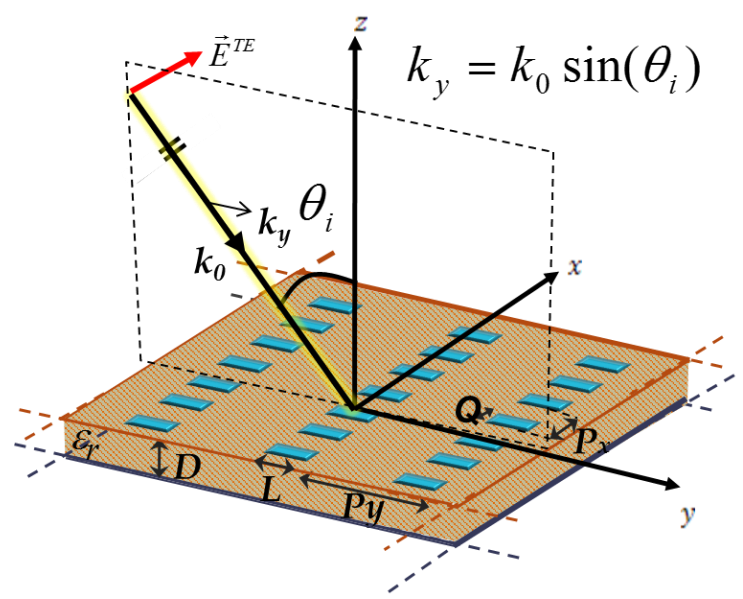

(a)

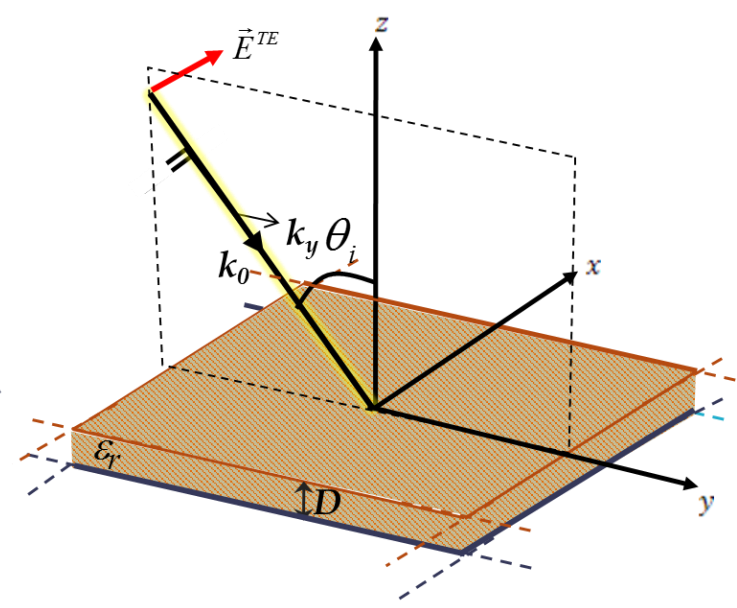

(b)

Fig. 2.5.2 Compared scenarios to obtain the poles and zeros of the $Y_{F S S}$ for a TE incidence. TE plane wave impinging with incident angle $\left(\theta_{i}\right)$ on (a) an infinite two-dimensional FSS of metallic dipoles on a grounded substrate and (b) a grounded substrate.

In order to obtain the poles and zeros of the polynomial, firstly the reflection phase is computed from the scenarios depicted in Fig. 2.5.2 by some full-wave plane-wave analysis (e.g., the Method of Moments (MoM), or FEM employed by some commercial full-wave simulation tools such as CST or HFSS ([CST 2010], [HFSS 2011])). In the scenario of Fig. 2.5.2(a), it is computed the reflection phase of the FSS printed on a dielectric substrate on a ground plane. In the scenario of Fig. 2.5.2(b), the grounded substrate is analyzed in the absence of the FSS. After some simulations, the identification of the poles and zeros of $Y_{F S S}\left(k_{y}, w\right)$ is performed. The identification of two poles and two zeros is illustrated in Fig. 2.5.3. To this aim, special emphases is now focused on the phase of the reflection coefficients of the $\operatorname{FSS}\left(\varphi_{F S S}\right)$, (solid blue line) and the grounded substrate $\left(\varphi_{S}\right)$ (black dotted-line). Observing the graph it can be seen that the poles of the $Y_{F S S}$ corresponds to the frequencies in which $\varphi_{F S S}=180^{\circ}$, what means that the FSS acts as a short-circuit, and so the admittance will be infinite at those frequencies. On the other hand the zeros of the polynomial correspond to the frequency points where both phases coincide $\left(\varphi_{F S S}=\varphi_{S}\right)$, which means the FSS is invisible to the incident wave. This is equivalent to an open circuit, so $Y_{F S S}$ is equal to zero at these frequencies, as expected. The identification process is repeated for a set of discrete angles of incidence $\theta_{i}$. Thus, a matrix of poles and zeros of the polynomials for different discrete simulated $\theta_{i}$ is obtained. 


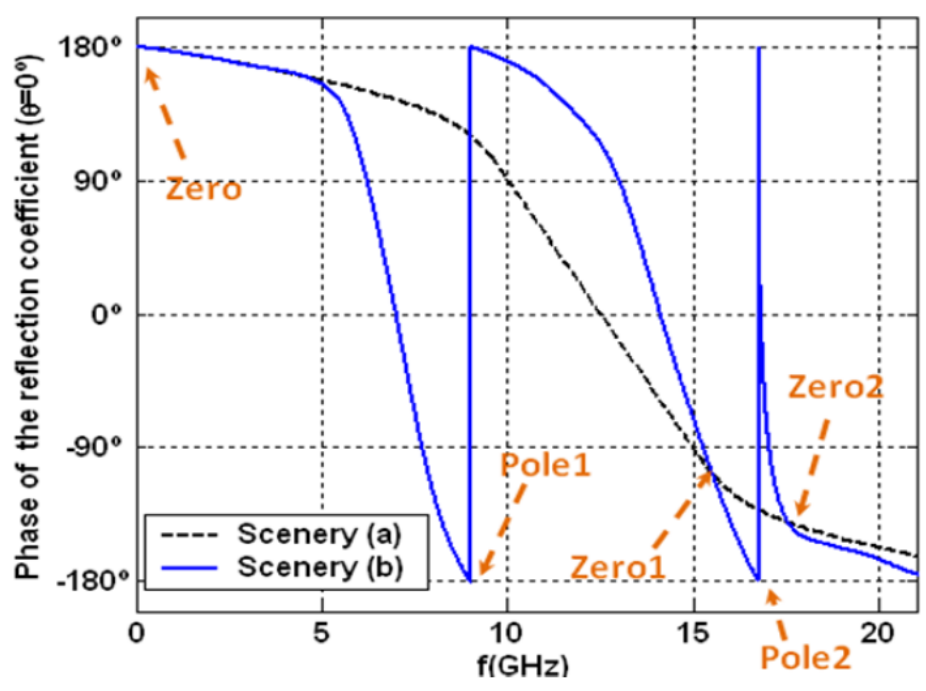

Fig. 2.5.3 Phase of the reflection coefficient under normal incidence $\left(\theta_{i}=0\right)$.

In order to calculate $Y_{F S S}$ for any incidence angle or frequency value, an interpolation of the poles and zeros with the longitudinal propagation constant ( $k_{y}$ in this TE incidence example), or the angle of incidence $\left(\theta_{i}\right)$, is performed. The relation between the poles and zeros with respect to $k_{y}$ is usually very soft, being easily interpolated by a polynomial of grade 1 or 2 . This is also advantageous, because with just a few full-wave simulations for some discrete incidence angles, the FSS response can be accurately characterized.

The Maci's pole-zero method supposes an attractive solution because of its simplicity and high applicability, but it is limited to the characterization as a function of frequency. For dispersion analysis as a function of physical dimensions of the FSS, a modified pole-zero technique was proposed in [García-Vigueras 2010] in order to obtain a closed expression of $Y_{F S S}$ as a function of the length of the dipoles of the FSS ( $L$ in Fig. 2.5.2) for a fixed frequency of interest. This method, which is derived from the Maci's original pole-zero method, proposes a similar polynomial expression to characterize the admittance:

$$
Y_{F S S}\left(k_{y}, L\right)=j \frac{L\left[L-L_{z 1}\left(k_{y}\right)\right] \ldots\left[L-L_{z m}\left(k_{y}\right)\right]}{\left[L-L_{p 1}\left(k_{y}\right)\right] \ldots\left[L-L_{p n}\left(k_{y}\right)\right]}
$$

A simple comparison between the above expression with Eq.(2.5.11) reveals that the dependence of $Y_{F S S}$ on $L$ in Eq.(2.5.12) is assumed to be similar to that with frequency. This similarity comes from the fact that the dipoles are resonant in the direction of their length, and therefore both variables are expected to have a similar influence on the behavior of $Y_{F S S}$. Thus, the task of obtaining an expression for the equivalent admittance as a function of $L$ can then be also reduced to extracting a set of poles and zeros (which are assumed to vary slowly with the angle of incidence as occurred with frequency in [Maci 2005]). 
In this case, the reflection coefficient experienced by an incident plane wave on the FSS printed on a grounded dielectric substrate (as shown in Fig. 2.5.2(a)) or on midair, depending if the FSS is employed for a PRS or a HIS, is obtained for different dipole lengths, $L$, and some angles of incidence, at the frequency of interest. From these full-wave results, the values of the admittance $Y_{F S S}$ can be obtained by some simple transmission lines, as shown later. With the resulted values of the admittance, the identification of the poles and zeros in this case is performed directly from rational fitting by any of the standard procedures available in the literature; in this case it is used a least squares scheme implemented in Matlab ([Matlab 2012]). In next subsection, it is shown how this method has been employed to characterize the PRS as a function of its resonant length $\left(L_{P R S}\right)$, for the design frequency of interest $(5.6 \mathrm{GHz})$.

In order to characterize the tunable HIS loaded with varactors employed in this thesis, the modified pole-zero method has also been successfully applied for first time. In this case, $Y_{F S S}$ is obtained as a function of $C_{j}$. The polynomial assumed for $Y_{F S S}$ as a function of $C_{j}$ has been expressed as

$$
Y\left(k_{y}, C_{j}\right)=j \frac{\left[C_{j}-C_{j_{z 1}}\left(k_{y}\right)\right] \ldots\left[C_{j}-C_{j_{z n}}\left(k_{y}\right)\right]}{\left[C_{j}-C_{j_{p 1}}\left(k_{y}\right)\right] \ldots\left[C_{j}-C_{j_{p 1}}\left(k_{y}\right)\right]}
$$

Where it can be observed that the assumption of a zero at the origin $\left(C_{j}=0\right)$ is not contemplated. Previous methods assumed a zero at the origin for physical reasons. When frequency is null, the admittance response is equivalent to an open/short circuit (e.g. case of dipole-based FSS or its complementary (slot-based FSS) respectively). Also, in the case of the dipole-based FSS, when $L=0 \mathrm{~mm}$, no dipoles exist, so $Y_{F S S}$ can be considered as an open circuit. However, when $C_{j}$ is null in the case proposed here, the varactors are equivalent to an open circuit, but the incident wave which impinges on the tunable HIS, still sees an array of metallic patches with dimensions $L_{H I S}$ x $Q_{H I S}$ (Fig. 2.4.1), on the grounded dielectric substrate. For the extraction of the poles and zeros, the same methodology employed in [García-Vigueras 2010], has been employed. In Subsection 2.5.4 it is demonstrated the validity of this re-adapted method.

Finally, note that these methods here introduced can be performed for either TE or TM incidence, by performing the full-wave plane-wave incidence analysis by TE or TM incidence. This analysis will depend on the type of leaky mode (TE or TM) considered in the structure. For the antenna presented in this chapter only TE analysis is needed, however, in Chapter 4, where a 2D FP LWA is presented, both TE and TM analysis of the PRS and tunable HIS will be required to analyze the propagation of TE and TM leaky modes in the main directions of the $2 \mathrm{D}$ structure. 


\subsubsection{Design and Characterization of the Passive PRS}

To construct the PRS, a FSS made of metallic resonant patches have been periodically arranged along the longitudinal direction of the antenna ( $y$-axis), placed on a dielectric substrate. As explained in [García-Vigueras 2011-I], the resonant length ( $L_{P R S}$ in Fig. 2.4.1(a)) of these patches must be properly chosen to tune the PRS reflectivity in order to control the leakage rate $\left(\alpha_{y}\right)$ of the leaky mode. So it will be desirable to analyze its transparency to control the antenna radiation efficiency. The rest of the geometrical parameters of the PRS are fixed. The width of the patches is $Q_{P R S}=18 \mathrm{~mm}$. The periodicity of the array is $P_{P R S}=20 \mathrm{~mm}, a=25 \mathrm{~mm}$ (width of the PPW), and the FSS is printed on FR4 substrate ( $\left.\varepsilon_{r P R S}=4.4, \tan \delta=0.018, D_{P R S}=0.8 \mathrm{~mm}\right)$.

In order to analyze the transparency of the PRS as a function of $L_{P R S}$ for a fixed frequency of $5.6 \mathrm{GHz}$, the previously described modified pole-zero method ([García-Vigueras 2010]) has been applied to extract the poles and zeros of the PRS equivalent admittance $\left(Y_{P R S}\left(k_{y}, L_{P R S}\right)\right)$. In this case, the equivalent admittance presented in Eq.(2.5.12) is expressed as a function of $L_{P R S}$ :

$$
Y_{P R S}\left(k_{y}, L_{P R S}\right)=j \frac{L_{P R S}\left[L_{P R S}-L_{P R S_{z 1}}\left(k_{y}\right)\right] \ldots\left[L_{P R S}-L_{P R S_{z n}}\left(k_{y}\right)\right]}{\left[L_{P R S}-L_{P R S_{p 1}}\left(k_{y}\right)\right] \ldots\left[L_{P R S}-L_{P R S_{p n}}\left(k_{y}\right)\right]}
$$

where it is observed that the poles and zeros of the polynomial are dependent on the propagation constant $\left(k_{y}\left(\theta_{i}\right)\right)$ of the plane wave, as expected.

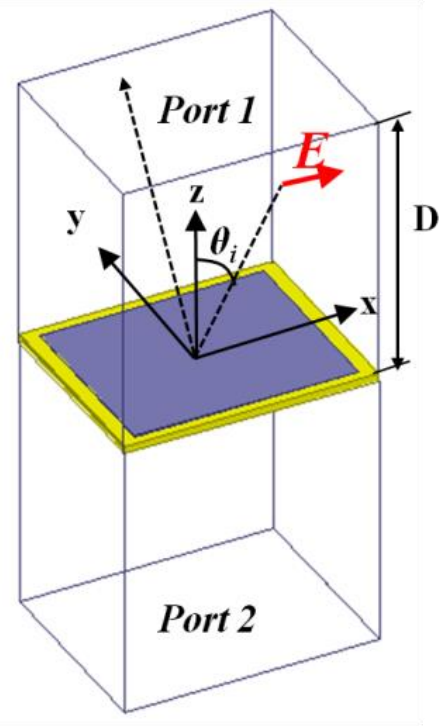

(a)

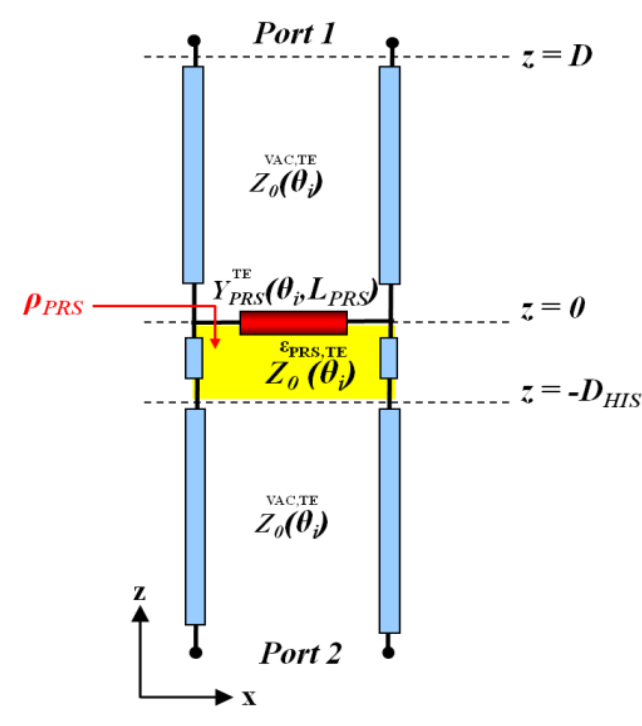

(b)

Fig. 2.5.4 (a) Simulation set-up to perform the plane wave incidence analysis on the PRS unit cell (Periodic Boundary Conditions (PBCs) are considered in the $\pm x$ and $\pm y$ directions). (b) Transmission line analyzed to extract the PRS admittance from the full-wave simulations. 
To apply this method, the characterization methodology described in previous subsection has been applied. In this case, the plane wave incidence analysis is performed by a full-wave technique based on the Method of Moments (MoM). This analysis aims to obtain the scattering response of the PRS when a plane wave impinges on it with different incidence angles $\theta_{i}$. A single PRS unit cell is considered (patch + dielectric FR4 substrate) suspended in midair, as can be observed in the schematic represented in Fig. 2.5.4(a). Periodic boundary conditions are imposed on the $x$ and $y$ directions, and two ports (at the top and at the bottom of the air box respectively) are defined to excite the plane wave with different incidence angles (see Fig. 2.5.4(a)). As the dispersion analysis is performed for TE modes of the FP structure, a horizontally x-polarized plane wave is launched in order to obtain the reflection coefficient of the FSS, propagating along the $y$ direction. The $Y Z$ plane will be the incidence plane which contains the wave vector of the impinging wave. The parametric simulations were run for a range of $L_{P R S}=[20-25] \mathrm{mm}$ in steps of $1 \mathrm{~mm}, 5$ incidence angles: $\theta_{i}=\left[0^{\circ}, 20^{\circ}, 40^{\circ}, 60^{\circ}, 80^{\circ}\right]$ and keeping a fixed frequency of $5.6 \mathrm{GHz}$ (the frequency of interest). The full-wave admittance $Y_{P R S}$ is readily obtained for every incidence angle from the resulted $S$ parameters and by analyzing the simple transmission line network depicted in Fig. 2.5.4(b)). Finally, the pole and zeros are extracted from the full-wave admittances by numerical fitting functions implemented in Matlab ([Matlab 2012]), as explained in the last subsection and in [García-Vigueras 2012-II]. In this case, the polynomial has been obtained with three zeros and two poles, as observed in Fig. 2.5.5(a) and (b) respectively. As occurs with the frequency dependence discussed in [Maci 2005], the values of the poles and zeros $\left(L_{P R S, P m}\left(k_{y}\right)\right.$ and $\left.L_{P R S, Z n}\left(k_{y}\right)\right)$, vary slowly with respect to the incidence angle ([García-Vigueras 2010]), what is convenient to interpolate these poles/zeros via a low order polynomial (in our case, we have performed a spline interpolation with Matlab). Therefore, a quasi-analytical expression for $Y_{P R S}$ is obtained as a function of the resonant patch length $L_{P R S}$ and $\theta_{i}$ (and hence $k_{y}$ ). In Fig. 2.5.6 it is illustrated the magnitude and phase of the reflection coefficient presented by the PRS ( $\rho_{P R S}$ in Fig. 2.5.4(b)) as a function of $L_{P R S}$ for a plane wave of $5.6 \mathrm{GHz}$ which impinges with three different incidence angles $\left(\theta_{\text {inc }}=\left[0^{\circ}\right.\right.$, $\left.30^{\circ}, 50^{\circ}\right]$ ). In order to assess the accuracy of the pole-zero analytical expression, the scattering response has been obtained by employing the PRS equivalent admittance $Y_{P R S}\left(k_{y}, L_{P R S}\right)$ and compared with the response obtained from full-wave MoM. Very good agreement is observed in the whole range of $L_{P R S}$, noting that even for angles not considered during the admittance extraction process $\left(\theta_{i n c}=\left[30^{\circ}, 50^{\circ}\right]\right)$, the prediction of the PRS response by the MoM and the analytical expression is practically the same, providing a maximum relative error of $5 \%$ with the use of 5 full-wave simulations (per angle of incidence). 


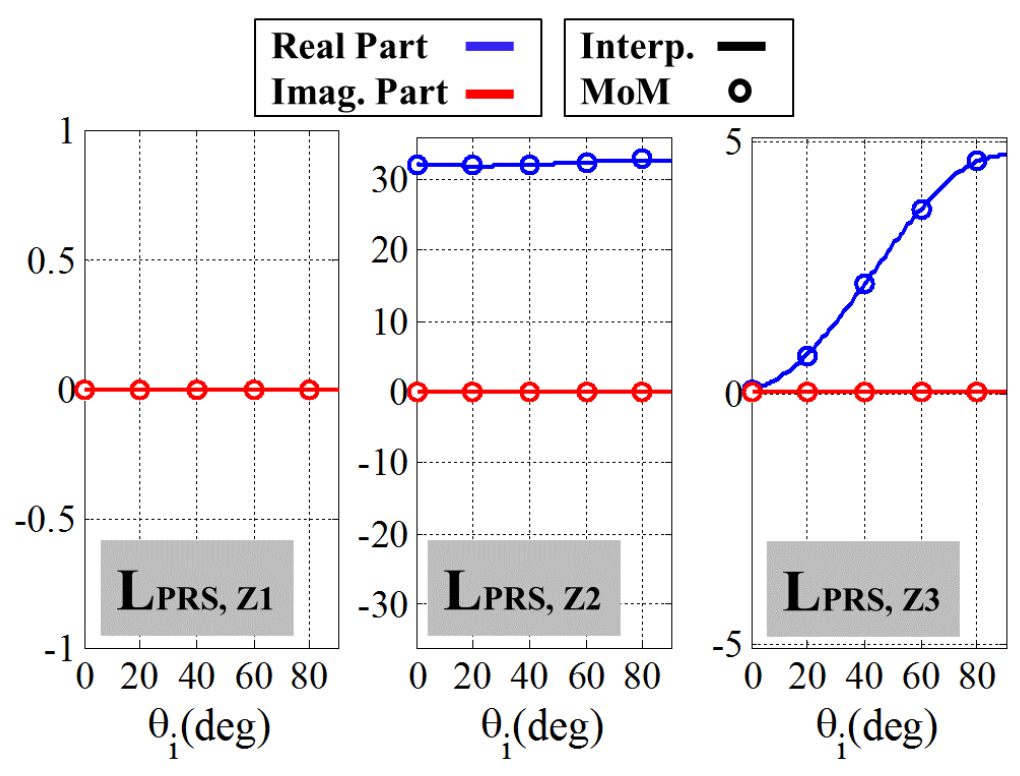

(a)

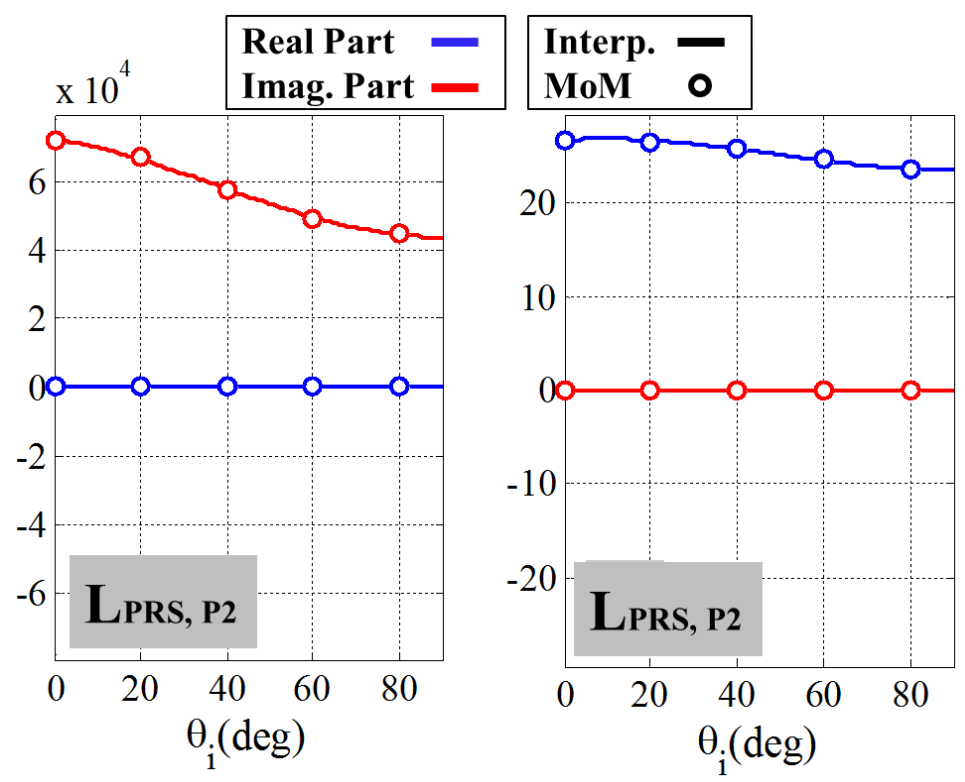

(b)

Fig. 2.5.5 Variation of (a) zeros and (b) poles of $Y_{P R S}$ with respect to the angle of incidence $\left(\theta_{i}\right)$.

To determine the proper value of the length of the patches for the final design of the prototype, a dispersion analysis with different values of $L_{P R S}$ has been performed. From these studies, it was chosen a resonance length of $L_{P R S}=22 \mathrm{~mm}$ in order to provide a high PRS reflectivity (low transparency) with $\left|\rho_{P R S}\right|>0.9$ for all scanning angles, and a reflection phase below $\phi_{P R S}<-158^{\circ}$, as shown in Fig. 2.5.6. As will see in future sections, this reflectivity of the PRS will ensure acceptable radiation efficiency for most of the scanning range achieved by the designed prototype in order to demonstrate the proof of concept. 


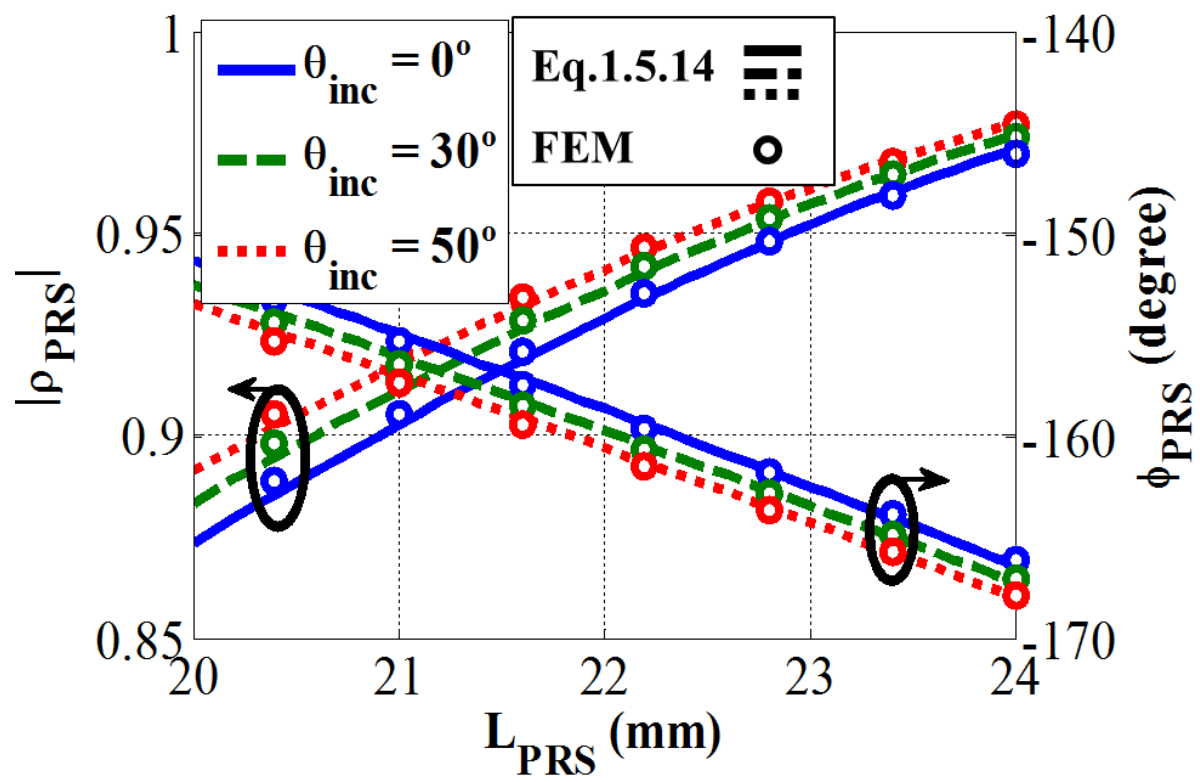

Fig. 2.5.6 Reflection coefficient of the PRS (magnitude and phase) seen by a plane wave for different incidence angles as a function of $L_{P R S}$ at $5.6 \mathrm{GHz}$. 


\subsubsection{Design and Characterization of the Tunable HIS}

The initial design of the tunable HIS employed in this thesis is a one-dimensional version of the structure considered in [Weily 2008]. The cell is basically a microstrip patch resonator with a tunable resonant frequency, as shown in Fig. 2.5.8(a). As the capacitance of the varactor diodes is varied, the resonant frequency of the patch antenna changes and so does its reflection phase. The patches are printed on a grounded dielectric substrate of Rogers RO4230 ( $\varepsilon_{r P R S}=3$, $\left.\tan \delta=0.0023, D_{H I S}=1.524 \mathrm{~mm}\right)$. The ground plane is made of aluminum. Dimensions of the patch are $L_{H I S}=14 \mathrm{~mm}$ by $Q_{H I S}=17 \mathrm{~mm}$, with a $g=1 \mathrm{~mm}$ air gap between its two halves. Spacing between adjacent cells is $P_{H I S}=30 \mathrm{~mm}$. Similar types of tunable HISs loaded with varactors have been employed before for reconfiguration purposes, such as the design of reconfigurable reflectors [Sievenpiper 2002], [Sievenpiper 2003], reconfigurable reflectarrays [Hum 2005, 2007], or tunable FSS [Mias 2007]. Also, an electronically tunable HIS was applied in [Sievenpiper 2005] to transform a surface-wave into a backward or forward leaky-wave, thus achieving beam scanning from a two-dimensional textured surface. In [Weily 2008], [Costa 2011], the same tunable HIS was applied to tune the operating frequency of low profile 2D FP LWAs, and similar reconfigurability was demonstrated in [Costa 2008] for a tunable HIS fed by a bow-tie antenna.

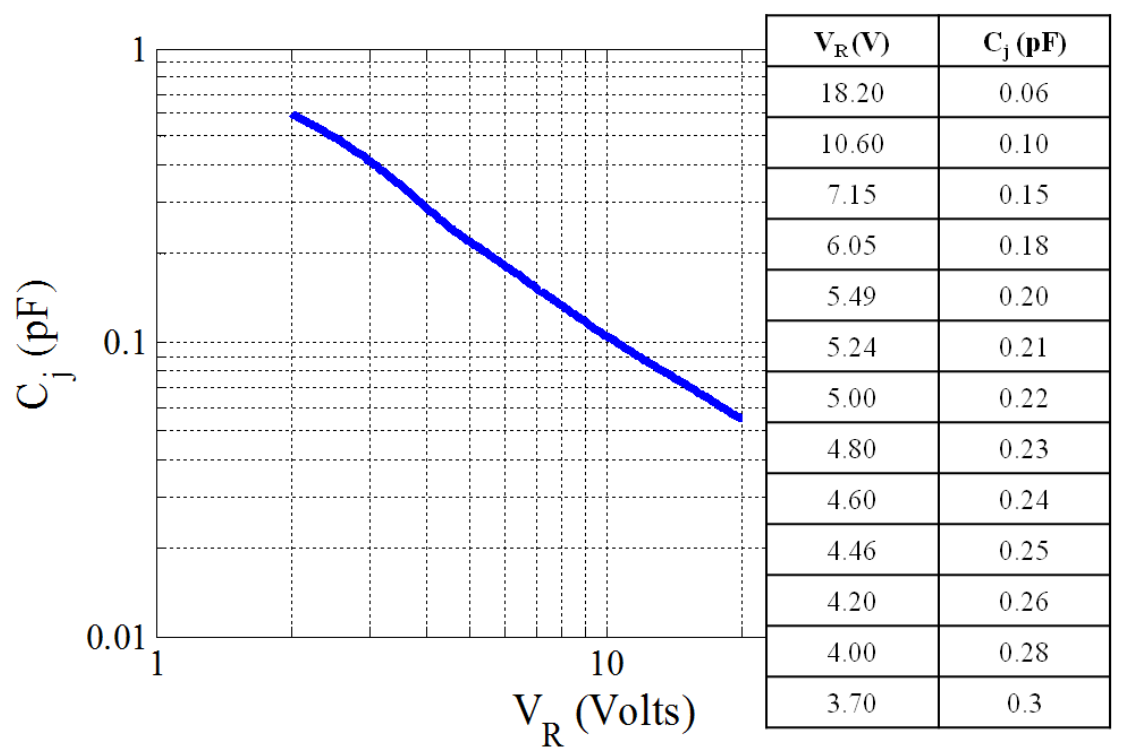

Fig. 2.5.7 Typical performance $V_{R}$ vs $C_{j}$ of Aeroflex Metelics MGV 125-08-0805-2 ([Aeroflex 2005]).

We have also chosen to use the same distributed element bias network employed in [Weily 2008] to enable the bias voltage to be applied to a complete row of phase agile cells, thus simplifying the control of the cells. Referring to Fig. 2.5.8(a) the bias network consists of two 
high impedance lines ( $0.5 \mathrm{~mm}$ wide) connected to a via-through (connected to the DC source) and a bypass capacitor. The electrical distance of the high impedance lines, from the edge of the patch to the vias, is a quarter wavelength at $5.6 \mathrm{GHz}$ so the vias appear as RF open circuits at the edge of the microstrip patch.

The GaAs hyperabrupt varactor diodes used in the tunable HIS are Aeroflex Metelics MGV 125-08-0805-2 (0805-2 package), which have a junction capacitance $\left(C_{j}\right)$ tuning range of $0.055 \mathrm{pF}$ to $0.6 \mathrm{pF}$ for a corresponding bias (tuning) voltage $\left(V_{R}\right)$ of 2 to $20 \mathrm{~V}$. The varactor's tolerance for $C_{j}$ is $\pm 0.05 \mathrm{pF}$, according to the manufacturer datasheet ([Aeroflex 2005]). Fig. 2.5.7 shows the typical performance ( $V_{R}$ vs $C_{j}$ ) for this model in particular. From this curve, it is clearly observed that this capacitance is intrinsically related to the reverse bias voltage $\left(V_{R}\right)$ applied to the diode, so that the varactor can be seen as an electronically tunable capacitance. Thus, the variation in $C_{j}$ will determine the resonant frequency of the HIS, and so it allows electronic reconfiguration of the reflection phase when $V_{R}$ is tuned.

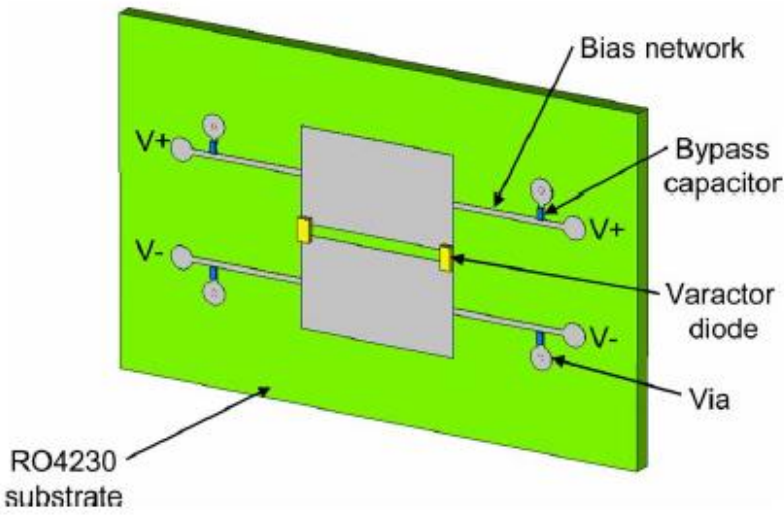

(a)

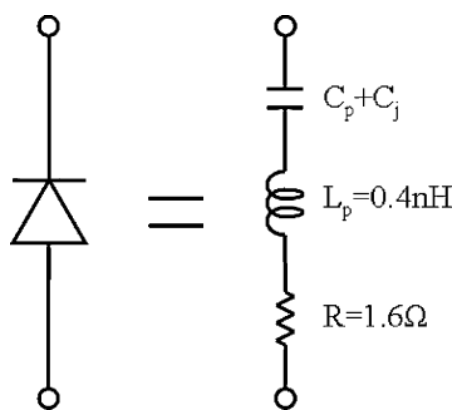

(b)

Fig. 2.5.8 (a) Configuration of the HIS unit cell employed to reconfigure the pointing angle of the reconfigurable antenna (Fig.3 in [Weily 2008]) and (b) Equivalent circuit of the varactor diode used to simulate the performance of the tunable HIS unit cell $\left(C_{p}=0.06 \mathrm{pF}, C_{j}=0.06 \mathrm{pF}\right.$ to 0.6pF) (Fig.4 in [Weily 2008])

A series RLC equivalent circuit (Fig. 2.5.8(b)) has been used to model the varactors in the fullwave simulation software employed to design the antenna ([CST 2010]). The model includes the diode parasitic inductance $\left(L_{p}\right)$ and series resistance $\left(R_{s}\right)$, while the total capacitance consists of both a parasitic capacitance and the junction capacitance $\left(C_{p}+C_{j}\right)$. A series RLC circuit has also been used to model the $2.2 \mathrm{pF}$ bypass capacitor $\left(C_{b}\right)$. For the bypass capacitor equivalent circuit, the parasitic inductance is $L_{b}=0.48 \mathrm{nH}$, while the series resistance is $R_{b}=1 \Omega$.

To check the RLC model reliability, tests were conducted in [Weily 2008] on a single unit cell prototype located in a WR-187 rectangular waveguide, comparing simulated ([CST 2010]) and 
measured responses of the cell for different tuning voltages. It is important to note that extract and check the model of the electronic tuning elements is considered a "must" for the proper design of reconfigurable antennas. In our case, the varactors have a deep effect over the final response of the antenna, so accurate design process might not be possible if the response of the varactors is not well modeled by the RLC circuit in the performed simulations.

Next, the characterization of the tunable HIS by an equivalent admittance $\left(Y_{H I S}\right)$ as a function of the junction capacitance $C_{j}$ is considered for a fixed frequency of $5.6 \mathrm{GHz}$. As aforementioned, the study of $C_{j}$ effect on the dispersion curves will be essential to predict the scanning response of the antenna. In this case, $Y_{H I S}$ will be extracted by applying the modified pole-zero method, but assuming a slightly different expression for the polynomial of the FSS admittance, as explained in previous subsection (Eq.(2.5.13)), which is expressed again below:

$$
Y_{H I S}\left(k_{y}, C_{j}\right)=j \frac{\left[C_{j}-C_{j_{z 1}}\left(k_{y}\right)\right] \ldots\left[C_{j}-C_{j_{z n}}\left(k_{y}\right)\right]}{\left[C_{j}-C_{j_{p 1}}\left(k_{y}\right)\right] \ldots\left[C_{j}-C_{j_{p 1}}\left(k_{y}\right)\right]}
$$

The process to extract the poles and zeros is similar to the performed for the PRS. A plane wave incidence has been performed for a single HIS unit cell at a fixed frequency of $5.6 \mathrm{GHz}$ (Fig. 2.5.9(a)) The simulations have been performed by a full-wave Finite Element Method (FEM) implemented in the commercial software CST ([CST 2010]) sweeping a discrete range of $C_{j}$ and $\theta_{i}$. In particular, the range of $C_{j}$ chosen to perform the simulations was $C_{j}=[0 \mathrm{pF}-0.4 \mathrm{pF}]$, in steps of $0.02 \mathrm{pF}$, and an incidence angle range of $\theta_{i}=\left[0^{\circ}, 20^{\circ}, 40^{\circ}, 60^{\circ}, 80^{\circ}\right]$. Fig. 2.5 .9 shows the simulation set-up and the transmission line network employed to extract the equivalent admittance from the scattering parameters $\left(S_{11}\right)$ obtained from the simulation results. It is worth to note that in order to characterize properly $Y_{H I S}$, we have evaluated the patch array on the same thin grounded substrate employed for the HIS in the design of the antenna. Due to the thin substrate $\left(D_{H I S}<<\lambda_{0}\right)$ employed to constitute the HIS structure, the HIS patches are in close proximity to the ground plane. This causes that the evanescent higher order Floquet harmonics, arisen from the periodic nature of the structure, strongly contribute to the scattered fields by the HIS, what affects to the FSS and the AMC resonance frequencies ([Sievenpiper 1999], [Goussetis 2006-I]). Thus, in order to obtain properly the equivalent admittance of the periodic structure from the full-wave results, this FSS must be simulated on the grounded substrate (with thickness $D_{H I S}$ ), in the same way which is operating inside the FP LWA. This phenomenon is not taken into account for the PRS, which is suspended in the middle of the FP cavity, far from the grounded plane or other structures which could affect its resonant response. 


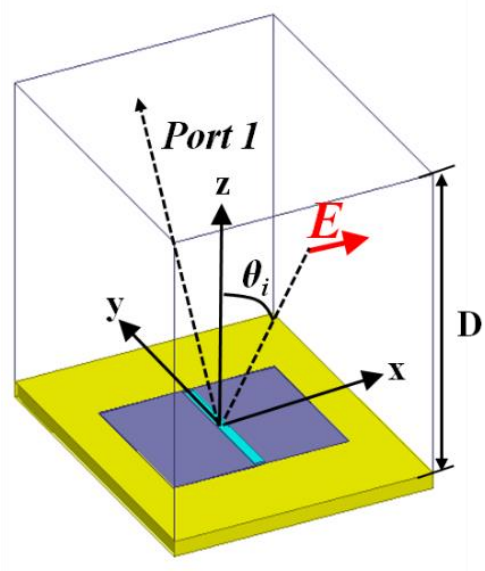

(a)

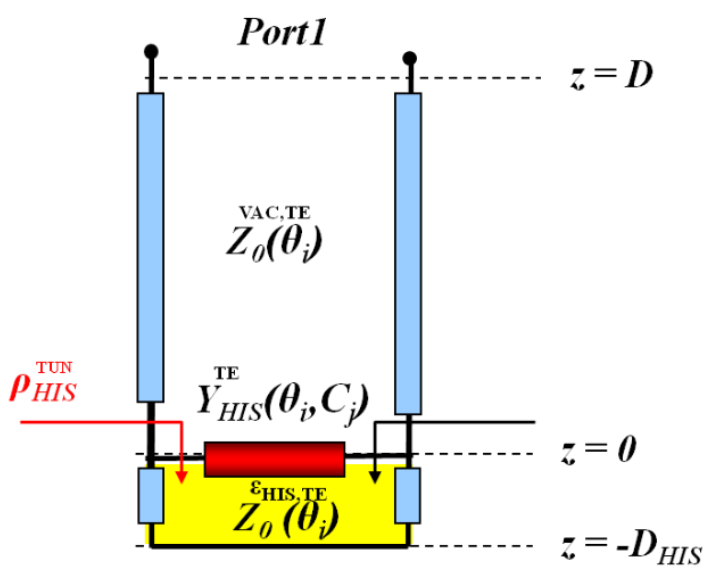

(b)

Fig. 2.5.9 (a) Simulation set-up to perform the plane wave incidence analysis on the tunable HIS unit cell (PBCs are considered in the $\pm x$ and $\pm y$ directions). (b) Transmission line analyzed to extract the HIS admittance from the full-wave simulations.

Fig. 2.5.10 shows the 3 zeros and 1 pole obtained to characterize $Y_{\text {HIS. }}$. Again, note that the poles and zeros of the polynomial are dependent on the angle of incidence of the leaky mode $\theta_{i}$, and hence on the propagation constant $k_{y}$ (also notice this dependence in the equivalent circuit used to extract the HIS admittance from full-wave simulations, depicted in Fig. 2.5.9(b)). Also, from Fig. 2.5.10(a) it can be observed that no zero corresponds to the origin $\left(C_{j}=0\right)$, as expected from our assumption. However, in previous section, $L_{P R S, Z 1}$ corresponded to $L_{P R S}=0 \mathrm{~mm}$, as also expected according to the variation of the $Y_{P R S}$ with $L_{P R S}$ ([García-Vigueras 2010).

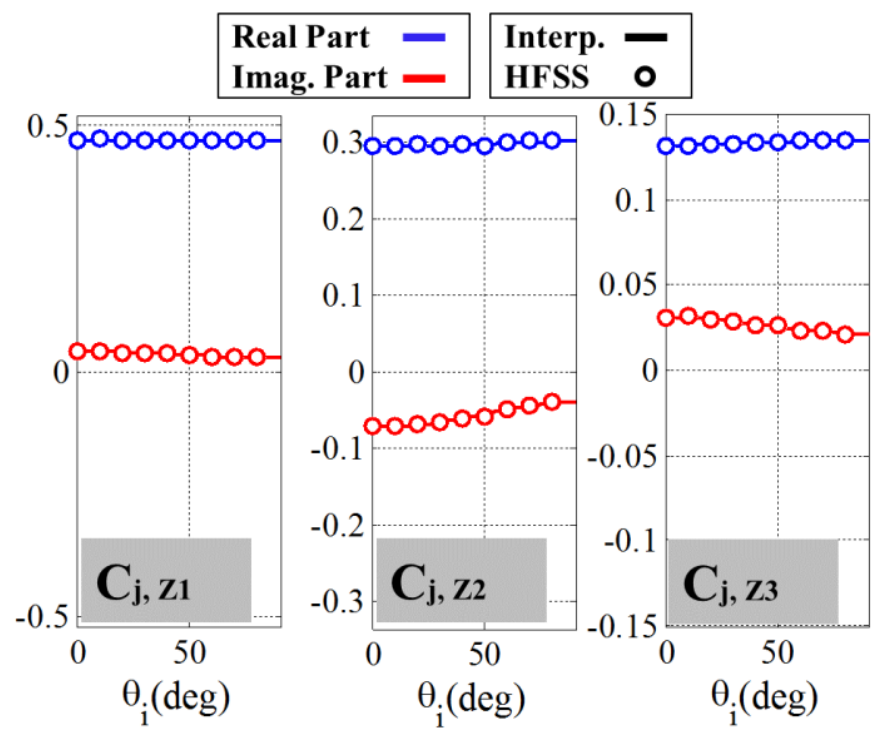

(a) 


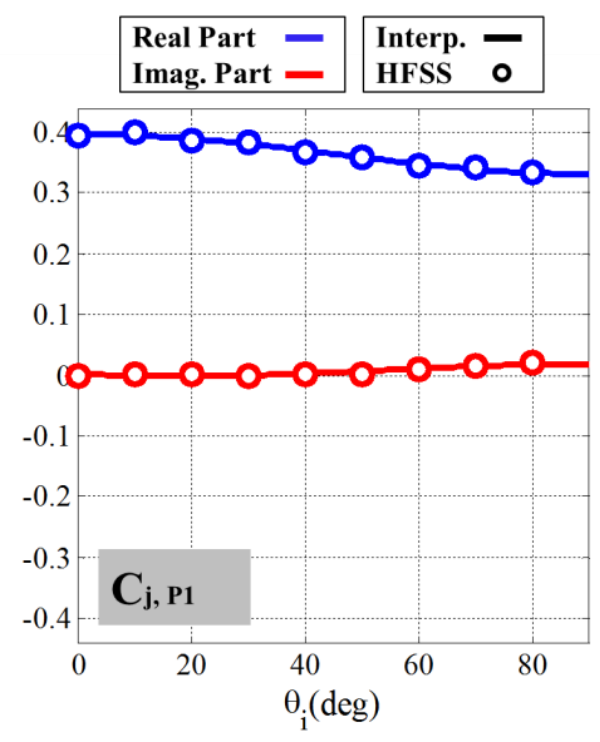

(b)

Fig. 2.5.10 Variation of (a) zeros and (b) poles of $Y_{H I S}$ with respect to the angle of incidence $\left(\theta_{i}\right)$.

This quasi-analytical expression models the response of the tunable HIS for any $C_{j}$ and $\theta_{i}$. As demonstrated in [García-Vigueras 2011-II], the boundary condition presented at the HIS interface changes drastically as the physical resonating length $L_{H I S}$ is varied, thus resulting in a direct mechanism to control the $\mathrm{TE}_{01}$ leaky-mode cut-off frequency and therefore the pointing angle at a fixed frequency. Yet, this control could only be mechanically achieved in [GarcíaVigueras 2011-II], replacing the whole PCB in order to vary $L_{H I S}$. The proposed reconfigurable LWA overcomes this disadvantage, since the effective resonant length of the HIS patches can be varied by properly modifying the varactors' junction capacitance $C_{j}$ ([Hum 2005]). This is shown in Fig. 2.5.11(a), where the reflection phase presented by the tunable HIS ( $\rho_{\text {HIS }}$ in Fig. 2.5.9(b)) provides PMC condition $\left(\phi_{H I S}=0^{\circ}\right)$ at different frequencies $(5.15 \mathrm{GHz}, 5.65 \mathrm{GHz}$ and $6.15 \mathrm{GHz})$ as $C_{j}$ is varied $(0.35 \mathrm{pF}, 0.23 \mathrm{pF}$, and $0.15 \mathrm{pF})$. These results were obtained using the pole-zero analytical expression of $Y_{H I S}$ as a function of frequency, obtained by the aforementioned pole-zero method proposed in [Maci 2005], for different values of $\theta_{i}$ and $C_{j}$. When the frequency is fixed at $5.6 \mathrm{GHz}$, the behavior of $Y_{H I S}\left(k_{y}, C_{j}\right)$ can be analytically modeled using (Eq.(2.5.13), obtaining the HIS reflection phase as a function of $C_{j}$ for different $\theta_{i}$ as shown in Fig. 2.5.11(b). Very good agreement with FEM results is observed for all the range of $C_{j}$ (from $0 \mathrm{pF}$ to $0.35 \mathrm{pF}$ ) and for all incidence angles. 


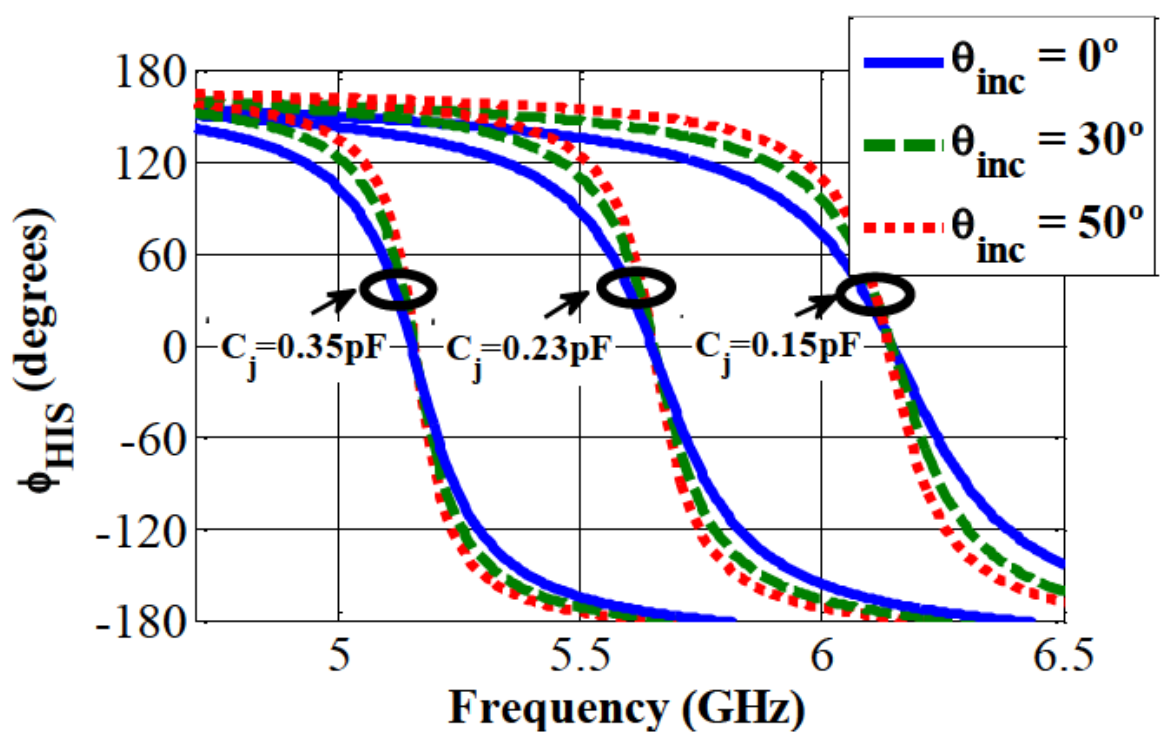

(a)

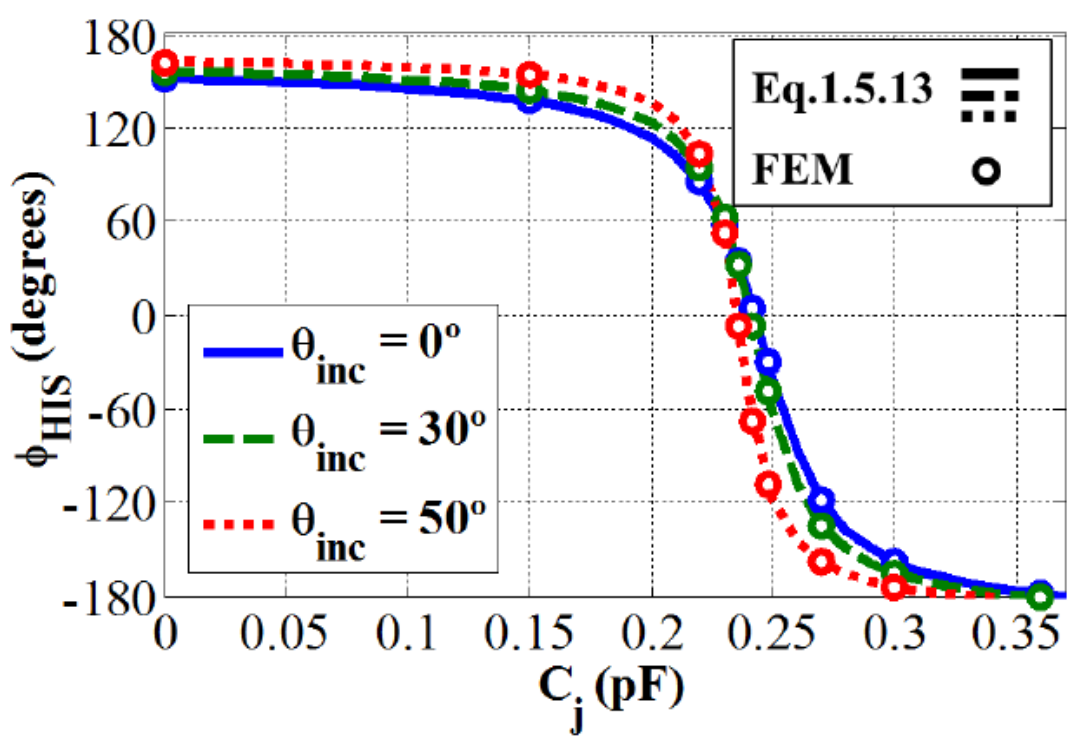

(b)

Fig. 2.5.11 Reflection phase of the tunable HIS seen by an incident plane wave for different incidence angles (a) as a function of frequency for different values of $C_{j}$ and (b) as a function of $C_{j}$ at $5.6 \mathrm{GHz}$.

Observing the phase variation with $C_{j}$, it can be noticed that the HIS nearly behaves as a grounded dielectric slab for $C_{j}=0 \mathrm{pF}\left(\phi_{H I S}=150^{\circ}\right)$. At this point the FSS patches can be considered almost "invisible" to the incident wave. For higher $C_{j}$, the HIS becomes a PMC sheet $\left(\phi_{H I S}=0^{\circ}\right)$ for $C_{j} \approx 0.23 \mathrm{pF}$, and as a PEC sheet $\left(\phi_{H I S}=180^{\circ}\right)$ for $C_{j}=0.35 \mathrm{pF}$. According to the results reported in [García-Vigueras 2011-II], increasing $C_{j}$ corresponds to a larger $L_{H I S}$, thus showing a direct relation between $C_{j}$ and the effective resonant length of the HIS patches. Since $C_{j}$ can be controlled by the varactors' reverse bias voltage ([Sievenpiper 2002], [Sievenpiper 2003], [Hum 2005], [Mias 2007], [Sievenpiper 2005], [Costa 2008], [Weily2008], [Costa 2011], electronic 
control of the pointing angle at a fixed design frequency should be possible by combining the passive 1D FP LWA and the tunable HIS. To theoretically confirm this fact, the behavior of the radiating leaky mode must be studied as a function of $C_{j}$. 


\subsection{Electronic Control of the Pointing Angle}

\subsubsection{Dispersion Analysis by TEN}

In this subsection, the effect of $C_{j}$ on the pointing angle $\theta_{R A D}$ and normalized radiation rate $\alpha_{y} / k_{0}$ is studied. Dispersion and attenuation relations of the TE leaky mode supported by the structure have been obtained as a function of frequency in Fig. 2.6.1. Note that these dispersion curves have been obtained from solving the dispersion equation (Eq.(2.5.1)) set out from the analysis of the TEN, which in this case includes the PRS and the HIS equivalent admittances as a function of frequency, extracted by the original pole-zero method presented in [Maci 2005]. Fig. 2.6.1(a) and Fig. 2.6.1(b) shows the frequency dispersion for the normalized phase $\left(\beta_{y} / k_{0}\right)$ and leakage $\left(\alpha_{y} / k_{0}\right)$ constants respectively as $C_{j}$ is varied from $0.1 \mathrm{pF}$ to $0.23 \mathrm{pF}$. From Fig. 2.6.1(a), it can be appreciated how the tunable HIS is able to tune the cutoff frequency of the Fabry-Pérot cavity. Thus, the $\mathrm{TE}_{01}$ leaky-mode cutoff frequency decreases as $C_{j}$ increases (defining the cutoff frequency of the leaky mode as the frequency which satisfies $\beta_{y}=\alpha_{y}$ [Jackson 2008]). Thus, the cutoff frequency is shifted down from $5.5 \mathrm{GHz}$, when $C_{j}=0.1 \mathrm{pF}$, till close to $5.2 \mathrm{GHz}$ when $C_{j}=0.23 \mathrm{pF}$. This frequency shift results in a continuous rise of the leaky-mode phase constant when $C_{j}$ augments at a fixed frequency, as shown in Fig. 2.6 .2 for $5.6 \mathrm{GHz}$.

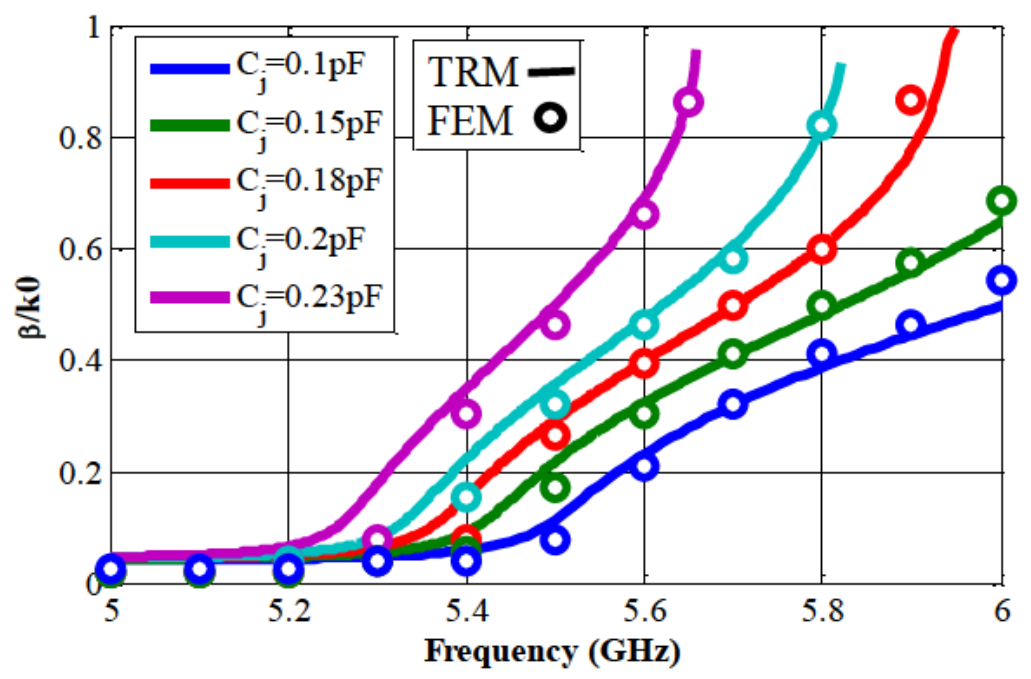

(a) 


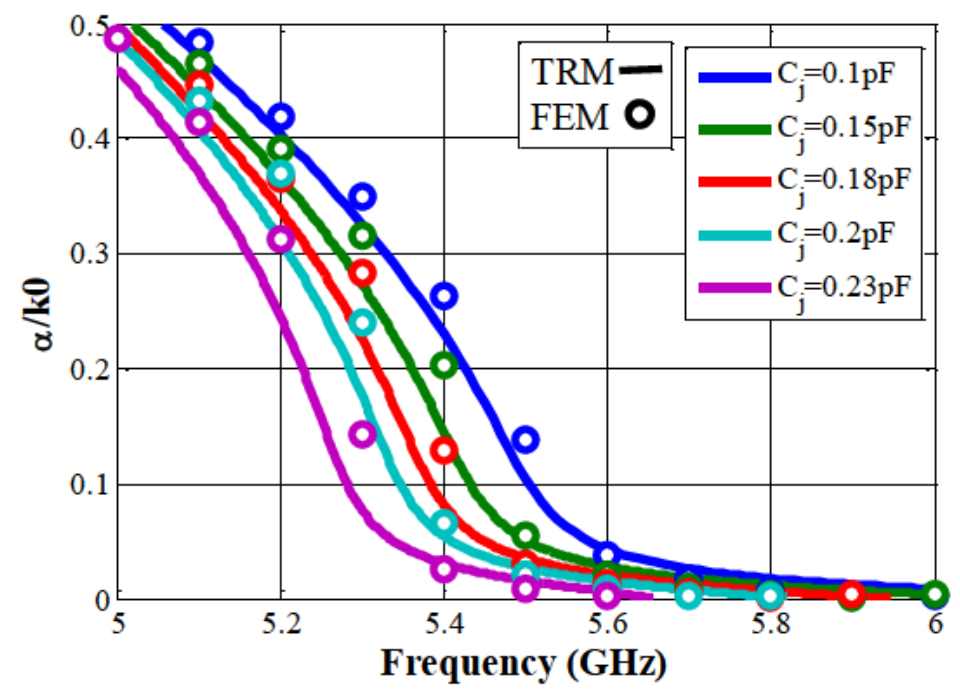

(b)

Fig. 2.6.1 Leaky-mode dispersion curves as a function of frequency for different cases of $C_{j}$ $\left(L_{P R S}=22 \mathrm{~mm}\right)$ a) Normalized phase constant $\left(\beta_{y} / k_{0}\right)$ and b) Normalized leakage rate $\left(\alpha_{y} / k_{0}\right)$.

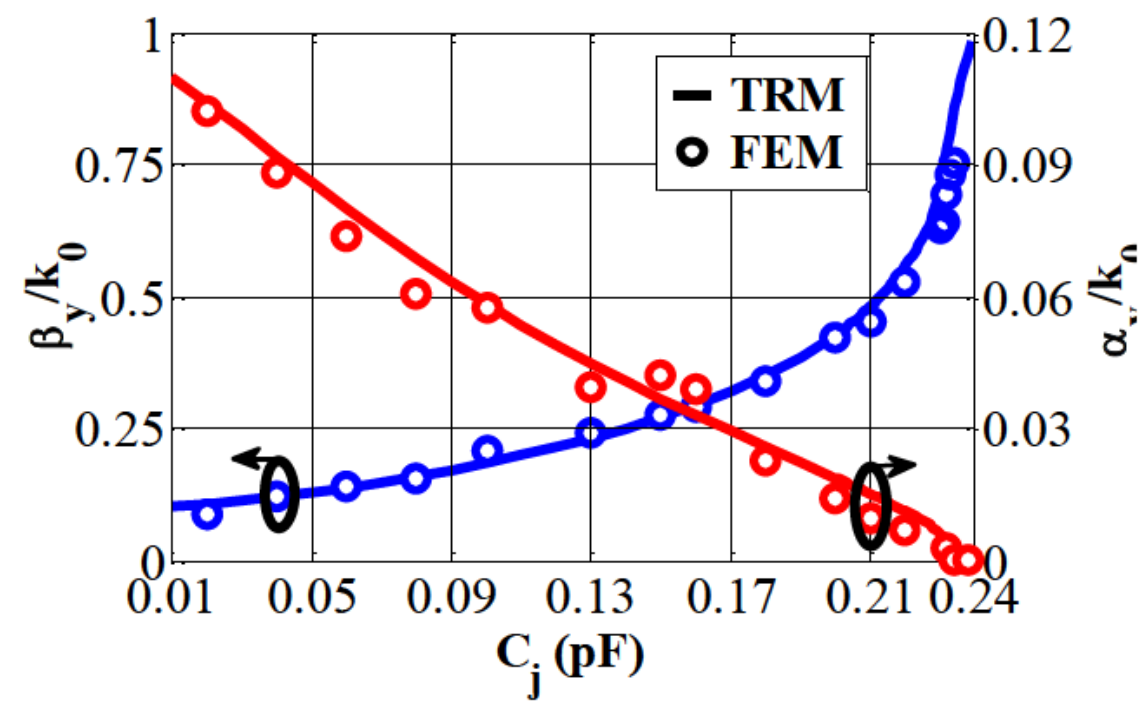

Fig. 2.6.2 Leaky-mode dispersion curves as a function of $C_{j}$ for a frequency of 5.6GHz.

Fig. 2.6.2 shows the dispersion and attenuation relations of the leaky mode for a fixed frequency of operation. These curves have been obtained from solving the dispersion equations derived from the TEN, which As it can be seen in Fig. 2.6.2, $\beta_{y} / k_{0}$ is varied from values close to zero when $C_{j}=0.01 \mathrm{pF}$, to values close to one when $C_{j}=0.24 \mathrm{pF}$, according to the predicted changes observed with the frequency response. Since the leaky-mode pointing angle is given by $\sin \theta_{R A D} \approx \beta_{y} / k_{0}$ (Eq.(2.2.4)), it is expected a theoretical fixed-frequency beam-scanning can be realized from almost broadside $\left(\theta_{R A D} \approx 0^{\circ}\right)$ to endfire $\left(\theta_{R A D}=90^{\circ}\right)$ by controlling $C_{j}$ in the aforementioned range $[0.01 \mathrm{pF}, 0.24 \mathrm{pF}]$. These theoretical results have been compared and validated with the dispersion curves obtained from full-wave FEM simulations ([CST 2010]) of 
a finite 3D model (also lossless) of the antenna with an aperture length of $L_{A}=5.2 \lambda_{0}$ at $5.6 \mathrm{GHz}$, which seem to agree quite well with theory. 


\subsubsection{Full-Wave Simulations}

To give more physical insight into the working principle of the proposed reconfigurable LWA, Fig. 2.6.3 illustrates the $\mathrm{TE}_{01}$ leaky-mode electric field distribution in the cross section of the simulated finite 3D model of the antenna ([CST 2010]) for different values of $C_{j}$ at the design frequency of 5.6GHz. For low values of $C_{j}$ the HIS behaves as a grounded slab (with no FSS), as concluded in the previous section from the HIS reflection phase response in Fig. 2.5.11(b), and the leaky-mode is resonating in the metallic cavity of height $H$, providing maximum horizontal field at $z=H / 2$, as it can be seen in Fig. 2.6.3 for $C_{j}=0.01 \mathrm{pF}$. However, as $C_{j}$ is increased to $0.23 \mathrm{pF}$, the HIS tends to behave as a PMC sheet which induces maximum field at its interface, as shown in Fig. 2.6.3. The boundary conditions seen by the leaky mode resonating in the FP cavity are strongly altered as $C_{j}$ is varied. As a result, the leaky-mode transverse wavelength $\lambda_{z}$ is modified from $\lambda_{z}=2 H$ when $C_{j}=0.01 \mathrm{pF}$ to $\lambda_{z}=4 H$ when $C_{j}=0.23 \mathrm{pF}$, as illustrated in Fig. 2.6.3. This enlargement in the transverse wavelength $\lambda_{z}$ implies the subsequent reduction in the longitudinal wavelength $\lambda_{y}$, which can be qualitatively appreciated in Fig. 2.6.4 where the longitudinal distribution of the electric field is shown for each case of Fig. 2.6.3 respectively. Therefore a rise in the leaky-mode longitudinal phase constant $\left(\beta_{y}=2 \pi / \lambda_{y}\right)$ as $C_{j}$ is increased is again demonstrated, as predicted by the results shown in Fig. 2.6.2.

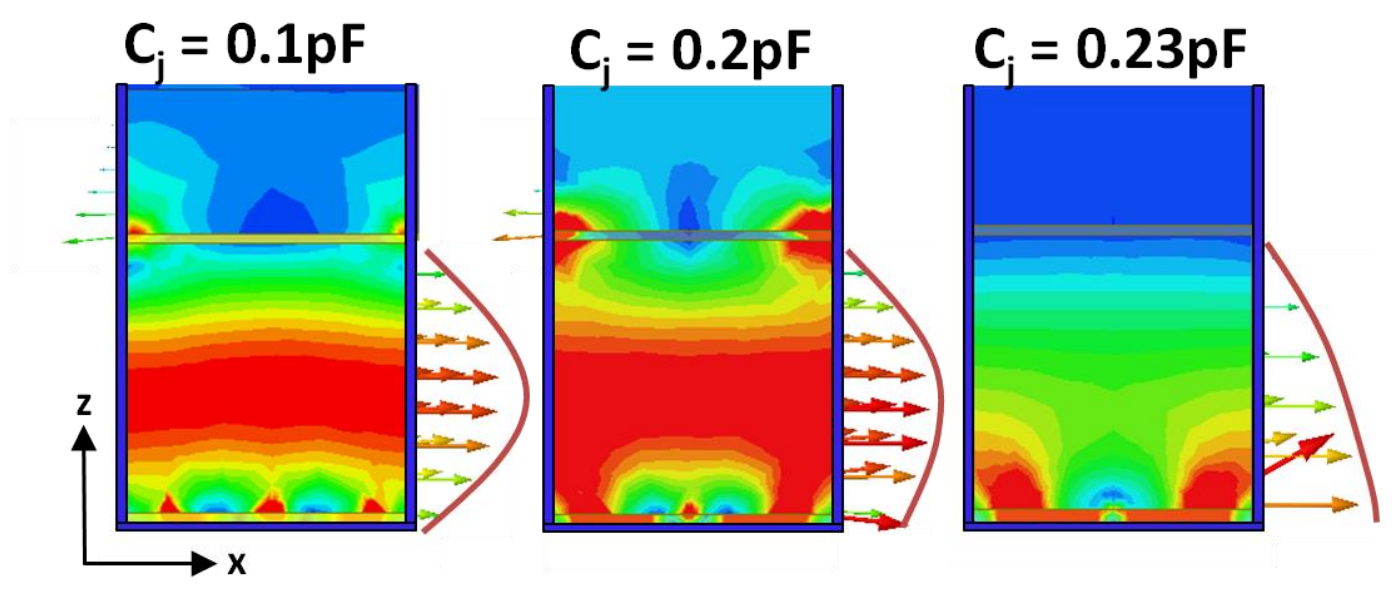

Fig. 2.6.3 Leaky-mode electric-field pattern at the transverse section of the reconfigurable FP PRS-HIS cavity at 5.6GHz for different values of $C_{j}$. Results obtained by FEM ([CST 2010]).
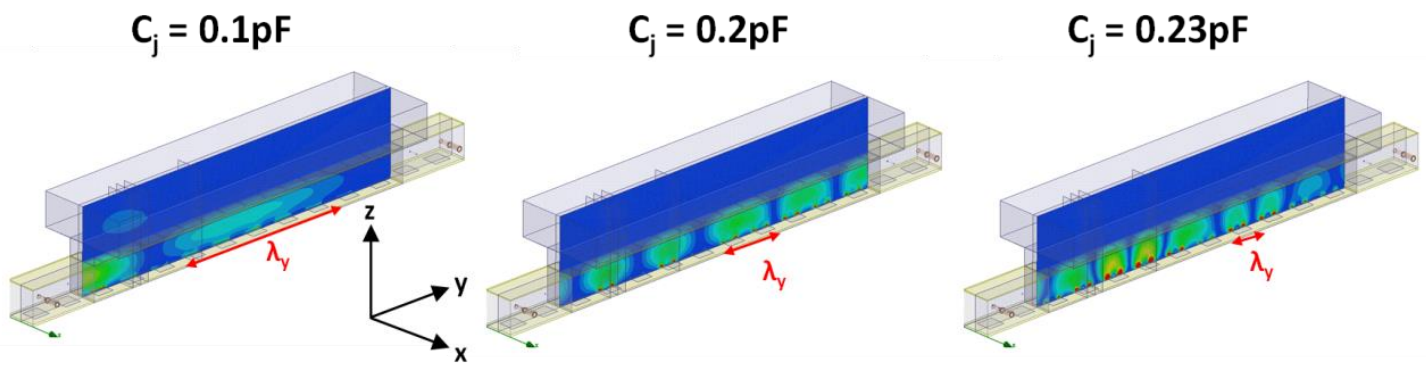
Fig. 2.6.4 Leaky-mode longitudinal electric-field distribution inside the reconfigurable FP PRS-

HIS cavity at $5.6 \mathrm{GHz}$ for different values of $C_{j}$. Results obtained by FEM ([CST 2010]).

Next, Fig. 2.6.5 shows the H-plane radiation patterns associated to different cases of $C_{j}$. Note that as the antenna is one-dimensional, and the radiated field $x$-polarized, its aperture field distribution defines a fan beam radiation pattern, as expected for any single onedimensional LWA, with the pattern narrow (and scanned with $C_{j}$ ) in the H-plane, and wide in the orthogonal E-plane. Once the leaky-mode complex propagation constant has been obtained as a function of $C_{j}$ by the TRM, the theoretical radiation patterns (solid line) for each $C_{j}$ case have been predicted for the finite structure applying a theoretical array factor approach ([Caloz 2004], [Oliner 2007]). The full-wave radiation patterns (dotted line) obtained by FEM ([CST 2010]) have been also shown to verify theory. As expected, the scanning angle $\theta_{R A D}$ is swept from nearly broadside towards the endfire direction as $C_{j}$ is increased, following the $\beta_{y} / k_{0}$ curve plotted in Fig. 2.6.2. Particularly, Fig. 2.6 .5 shows the results for $C_{J}=0.01 \mathrm{pF}\left(\beta_{y} / k_{0} \approx 0.09\right.$, $\left.\theta_{R A D} \approx 5^{\circ}\right), C_{j}=0.2 \mathrm{pF}\left(\beta_{y} / k_{0} \approx 0.42, \theta_{R A D} \approx 25^{\circ}\right)$, and $C_{j}=0.23 \mathrm{pF}\left(\beta_{y} / k_{0} \approx 0.65, \theta_{R A D} \approx 40^{\circ}\right)$.

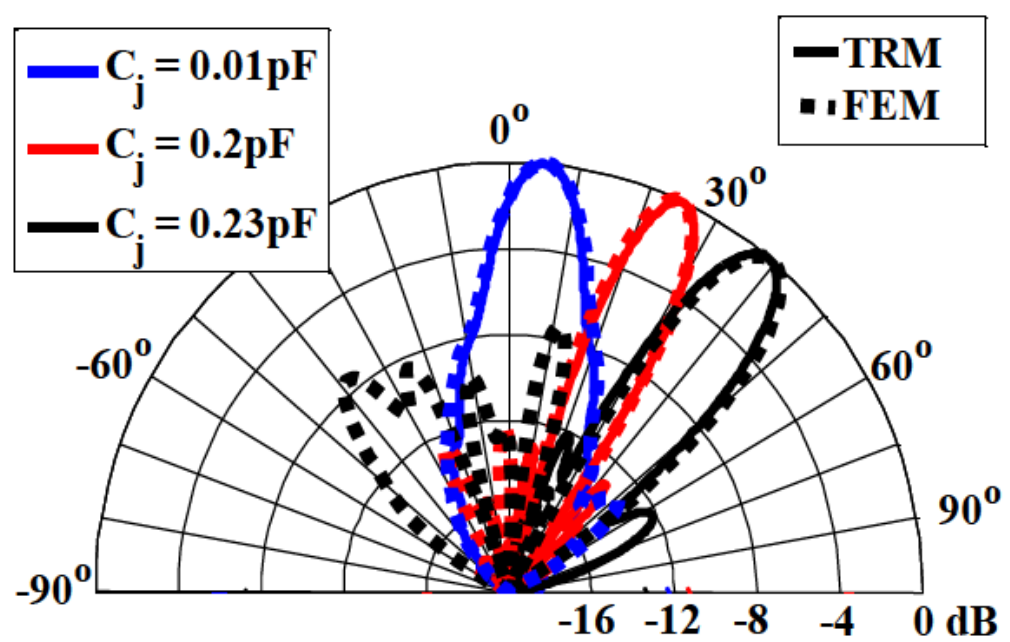

Fig. 2.6.5 Computed H-plane normalized radiation patterns for the proposed reconfigurable LWA at $5.6 \mathrm{GHz}$ for different $C_{j}$ for an antenna length $L_{A}=5.2 \lambda_{0}$. Theoretical array factor (solidlines) and simulations (dot-lines) represents the radiation patterns obtained from theoretical Array Factor approach.

The beam direction and the beamwidth predicted from theory using the TRM dispersion results are in very good agreement with full-wave FEM simulations performed with the finite 3D model. A strong reflected lobe appears for $C_{j}=0.23 \mathrm{pF}$, due to the very low leakage-rate associated with high values of $C_{j}$ (see Fig. 2.6.1(c)), which results in poor radiation efficiency and a large amount of energy being reflected at the far-end of the LWA. It is noteworthy that this fact will be one of the limiting factors which truncate the maximum scanning angle of the proposed reconfigurable LWA to angles below $40^{\circ}$. Also, it must be noticed that other limiting 
factors, such as the finite LWA aperture length, which affect the maximum directivity achievable as the scanning angle increases, or losses due to materials and varactors will be discussed in detail later. At this stage, the effect of losses due to the varactor and the substrates/metal losses has been ignored, being used a lossless model of the tunable HIS and the PRS for these preliminary full-wave simulations of the structure. These losses will be taken into account for the design of a fabricated prototype in subsequent sections. 


\subsubsection{Optimization of the Dynamic Scanning Range}

The physical height of the FP cavity $(H)$ is a key geometrical parameter which determines the scanning range and sensitivity of the proposed reconfigurable LWA. All previous results were computed using the optimum value $H=H_{\text {op }}=25.2 \mathrm{~mm}$. As can be seen in Fig. 2.6.6, a wider scanning range is obtained in this case, so that the cavity height makes the LWA minimum scanning angle $\left(\theta_{R A D} \approx 9^{\circ}\right)$ to be coincident with the minimum values of $C_{j}$ possible of the varactors $\left(C_{j} \approx 0.06 \mathrm{pF}\right)$. In this situation, the dynamic range of the varactors is fully used and the minimum scanning angle is close to broadside. On the other hand, as discussed in [GarcíaVigueras 2011-I], the maximum achievable pointing angle in this type of 1D LWA is produced when the HIS presents a PMC resonance (which in our case corresponds to a value close to $\left.C_{j}=0.23 \mathrm{pF}\right)$.

Above the HIS PMC resonance, a sudden fall of the leakage rate and a rapid divergence of the phase constant, as shown in Fig. 2.6.2, limit the scanning range to higher angles. Fig. 2.6.6 shows that for $H=H_{\text {opt }}$ the scanning angle can be swept from $\theta_{R A D}=5^{\circ}$ for $C_{j}=0.01 \mathrm{pF}$ to $\theta_{R A D}=40^{\circ}$ for $C_{j}=0.23 \mathrm{pF}$, as previously described. However, if $H>H_{\text {opt }}$, the minimum value of $C_{j}=0.01 \mathrm{pF}$ does not correspond to the leaky-mode cut-off point $\left(\theta_{R A D} \approx 0^{\circ}\right)$ but to a higher scanning state. As a result, the minimum scanning angle is located further from broadside $\left(\theta_{R A D}=30^{\circ}\right.$ at $C_{j}=0.01 \mathrm{pF}$ in Fig. 2.6.6 for $H=30 \mathrm{~mm}$ ). Since the maximum pointing angle given by the HIS PMC resonance $\left(C_{j} \approx 0.23 \mathrm{pF}\right)$ is $\theta_{R A D}=40^{\circ}$, the scanning range is reduced to $\left[30^{\circ}, 40^{\circ}\right]$ instead of $\left[5^{\circ}\right.$, $40^{\circ}$. Also, it can be observed that the sensitivity of $\theta_{R A D}$ with $C_{j}$ is lower for this case, due to the nonlinear response of the HIS reflection phase with respect $C_{j}$ (Fig. 2.5.11(b)).

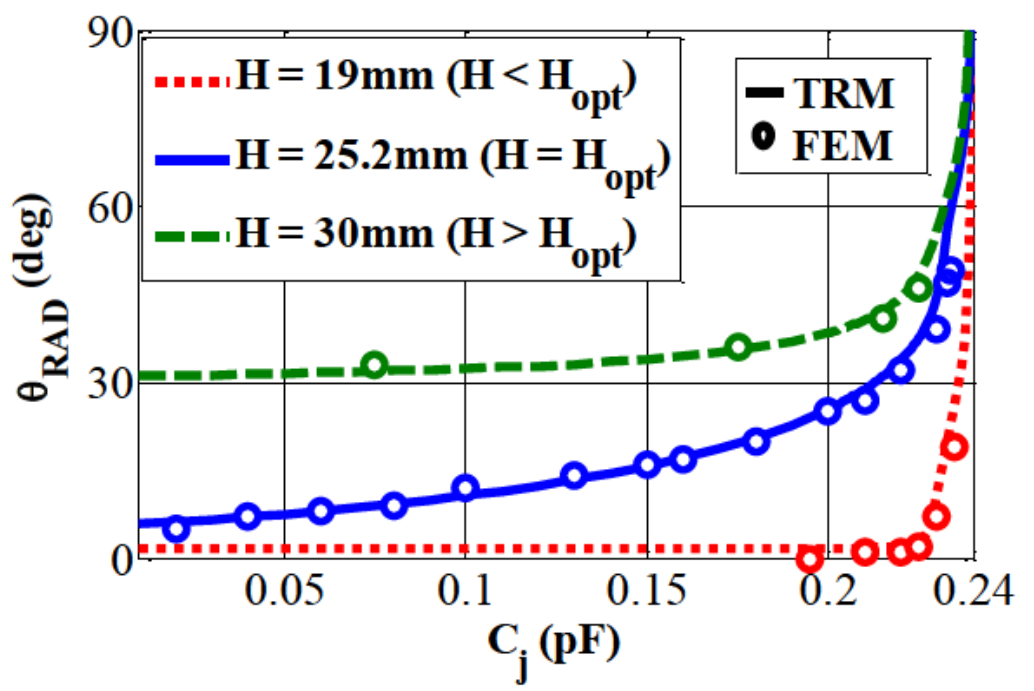

Fig. 2.6.6 Leaky-mode scanning-angle as a function of $C_{j}$ for different values of the Fabry-Perot cavity height, $H$, at $5.6 \mathrm{GHz}$. 
On the other hand, for shorter FP cavities $\left(H<H_{\text {opt }}\right.$, shown in dotted red line in Fig. 2.6.6) the leaky mode is below cutoff for most of the range of variation of $C_{j}$. As it can be seen in Fig. 2.6.6 for $H=19 \mathrm{~mm}$, the leaky modes are at cutoff region from $C_{j}=0.01 \mathrm{pF}$ to $C_{j}=0.2 \mathrm{pF}$, missing this region of the varactors' dynamic range. As a result, the scanning starts approximately above $C_{j}=0.2 \mathrm{pF}$, but then it is very soon limited to a maximum angle of $\theta_{R A D}=20^{\circ}$ at $C_{j}=0.23 \mathrm{pF}$, according to simulations. This reduces the scanning range and increases the sensitivity. Fig. 2.6.6 shows that the optimum cavity provides the most linear dependence between $\theta_{R A D}$ and $C_{j}$ and the highest scanning range. Once more, excellent agreement is found in the validation of the TRM dispersion curves and FEM results. 


\subsection{Experiments}

\subsubsection{Fabricated Prototype}

A reconfigurable antenna prototype has been designed to operate at 5.6GHz. Fig. 2.7.1(a) shows the 3D model of the final design model ([CST 2010]) where the real excitation and load have been already considered. Fig. 2.7.1(b) presents a perspective view of the fabricated prototype.

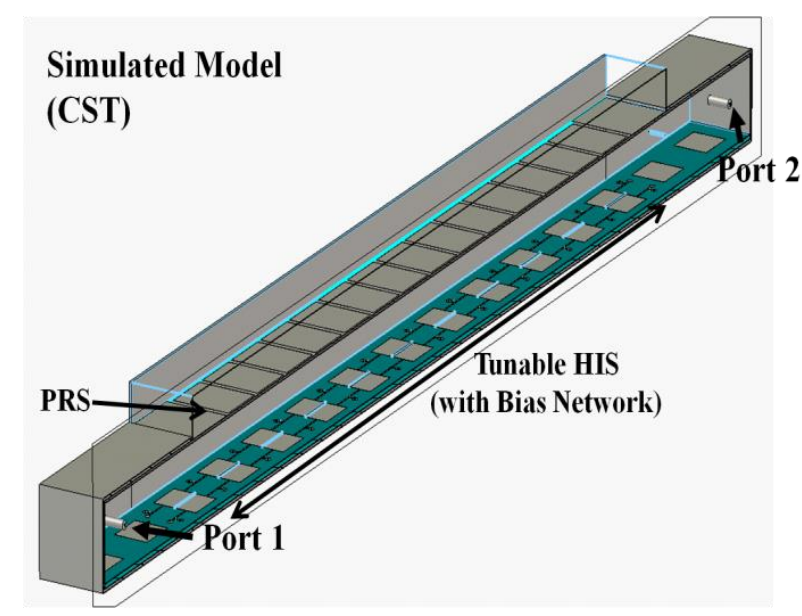

(a)

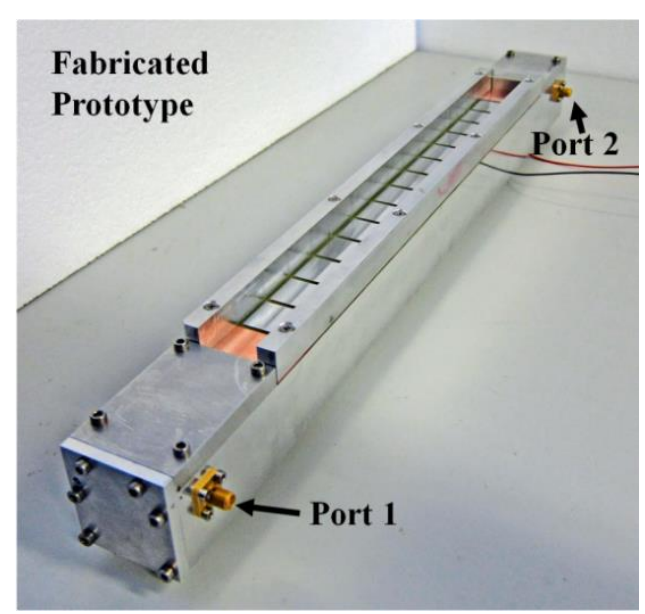

(b)

Fig. 2.7.1 (a) 3D Model of the final design of reconfigurable 1D FP LWA ([CST 2010]) and (b) Photograph of the manufactured reconfigurable LWA prototype.

The fabrication of the prototype and the experimental tests were carried out at CSIRO ICT Centre facilities located in Sidney (Australia). As illustrated in Fig. 2.7.2(a), three constituent parts in the reconfigurable LWA can easily be distinguished: a parallel-plate waveguide, a passive PRS printed-circuit, and a tunable HIS. The radiating aperture of the antenna has a length of $L_{A}=5.2 \lambda_{0}$ at $5.6 \mathrm{GHz}$. A detailed photograph of the 11 phase agile cell used for the tunable HIS, including the biasing network described above is depicted in Fig. 2.7.2(b). A total of 22 MGV125-08 varactor diodes (with 0805-2 package) were employed. They are welded to the two metallic pieces which compound each one of the HIS resonant patches, as can be appreciated in Fig. 2.7.2(b). In order to reduce the uncertainty of the antenna response introduced by the varactors' tolerances $( \pm 0.05 \mathrm{pF}$ according to the datasheet), the diodes were ordered "batch match" processed, which means that the manufacturer ensures the parameters of the diodes are within a given tolerance at a given bias voltage (at a slightly extra cost). To procure varactors with similar electrical response is an important issue to keep in mind for the fabrication process of these reconfigurable antennas. It should be stressed that the use of varactors with similar electronic response will avoid (or at least reduce the probability) the 
generation of phase aberration which spoil the antenna radiation pattern due to unwanted variations of the leaky-mode phase constant produced by a non-homogeneous response of the tunable HIS along the FP cavity of the LWA.

\section{Parallel Plates}

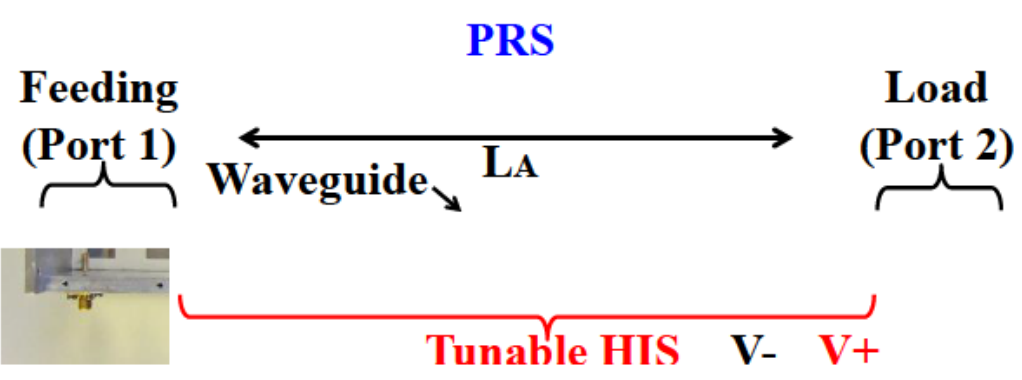

(a)

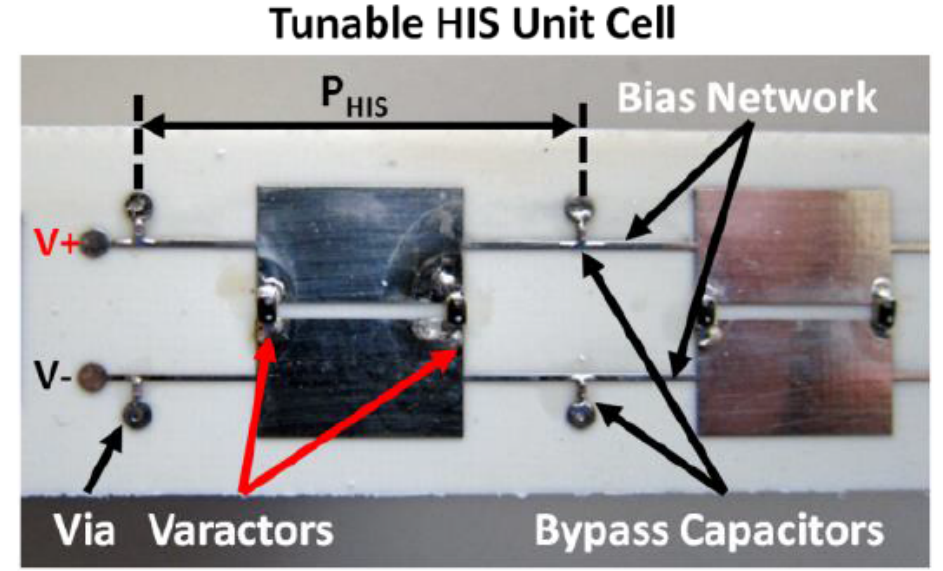

(b)

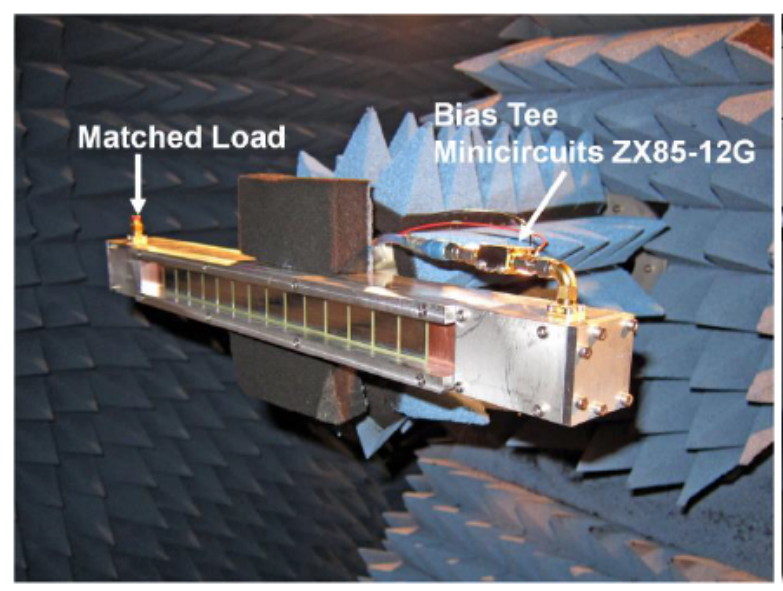

(c)

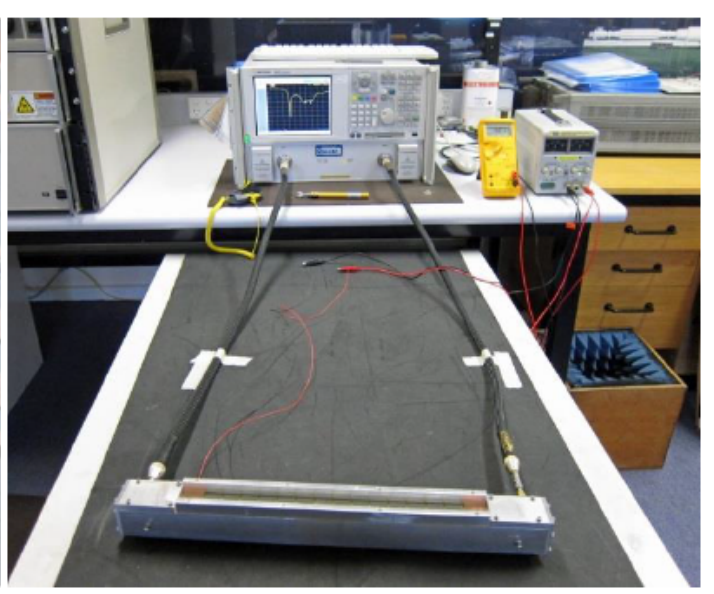

(d)

Fig. 2.7.2 Photographs of (a) Manufactured reconfigurable LWA prototype, (b) Tunable HIS phase agile cell, (c) Radiation pattern measurement experimental set-up and (d) S parameters measurements set-up. 
To excite the $\mathrm{TE}_{01}$ leaky-mode in the tunable 1D FP cavity, a coaxial probe-to-waveguide transition has been designed, similar to the reported in [García-Vigueras 2011-II]. However, due to the dynamic operation of this reconfigurable antenna, the structure design and optimization process required for designing this feed has been more laborious. Additional elements such as tuning screws have been included to add an additional degree of freedom, which allows to achieve better input matching for the dynamic range of interest. A midpoint of the antenna scanning range, $C_{j}=0.15 \mathrm{pF}$, which corresponds approximately to a scanning angle of $\theta_{R A D}=18^{\circ}$, was chosen to optimize the dimensions of the excitation. It is logical to think that if the excitation is matched for an intermediate operating point of the antenna, good performance will be ensured for the around operating points. Thereby, good matching across most of the dynamic range of $C_{j}$, has been obtained, as demonstrated with the analysis of the scattering parameters in next subsection. The schematic of the excitation structure is depicted in Fig. 2.7.3(a). A rectangular short-circuited waveguide with a horizontal coaxial probe inserted in the $x$-direction, perpendicular to the lateral wall of the waveguide, is designed in order to excite properly the $\mathrm{x}$ polarized $\mathrm{TE}_{01}$ mode. A coaxial hat has been added at the end of the inserted coaxial inner conductor in order to improve the bandwidth of the excitation (what also enhances the input matching at the operating points around). A tuning screw has been placed in the opposite wall at a certain distance from the coaxial in order to give an additional degree of freedom to the matching optimization process. This screw length has been optimized by simulation, but one advantage of including these screws is that they can provide fine adjustment of the real prototype later, in the test bench, in order to improve the input matching response experimentally. On the top of the waveguide, the same FR4 substrate employed for the PRS is located, shielded by the top waveguide wall. Concerning the height of the waveguide, because the FP cavity has a size lower than $\mathrm{H}=25.2 \mathrm{~mm}<\lambda 0 / 2$, the fundamental $\mathrm{TE}_{01}$ mode of the rectangular waveguide employed cannot be supported by a regular rectangular waveguide with such dimensions in a natural way (since the mode is below the cutoff frequency of the rectangular waveguide). Thus, a short passive (non-reconfigurable) HIS is introduced at the bottom of the waveguide, composed of two metallic patches on the HIS substrate. The dimensions of the patches ( $\mathrm{K}$ and $\mathrm{F}$ in Table 2.7.1) were calculated to match the input impedance of the rectangular waveguide when the antenna FP cavity is operating at the aforementioned point, $\mathrm{Cj}=0.15 \mathrm{pF}$. Thereby, it provides the necessary phase inside the cavity to allow propagation of the desired fundamental $\mathrm{TE}_{01}$ mode along this subwavelength waveguide, as observed by the colored arrows drawn at the output interface of the waveguide. A full-wave optimization process was performed to calculate the optimal dimensions of the coaxial-towaveguide transition. To this aim, the optimization tool integrated in the commercial software [CST 2010] was employed. The final optimized dimensions can be found in Table 2.7.1. 

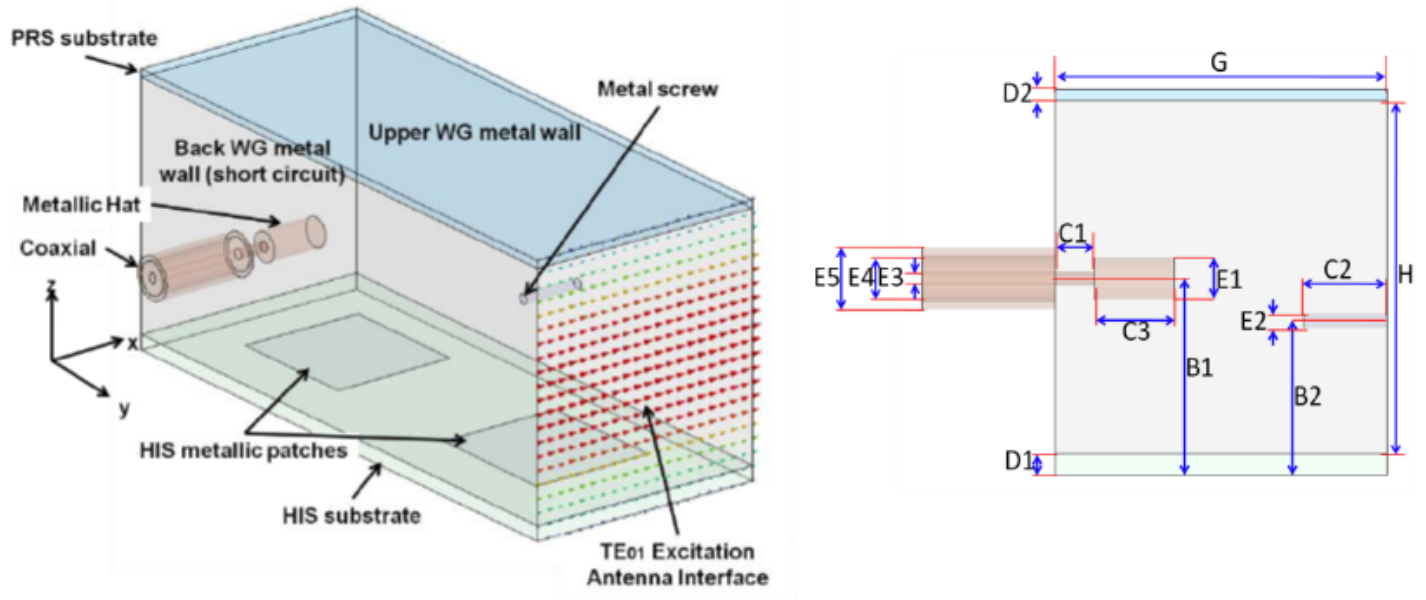

(a)

(b)
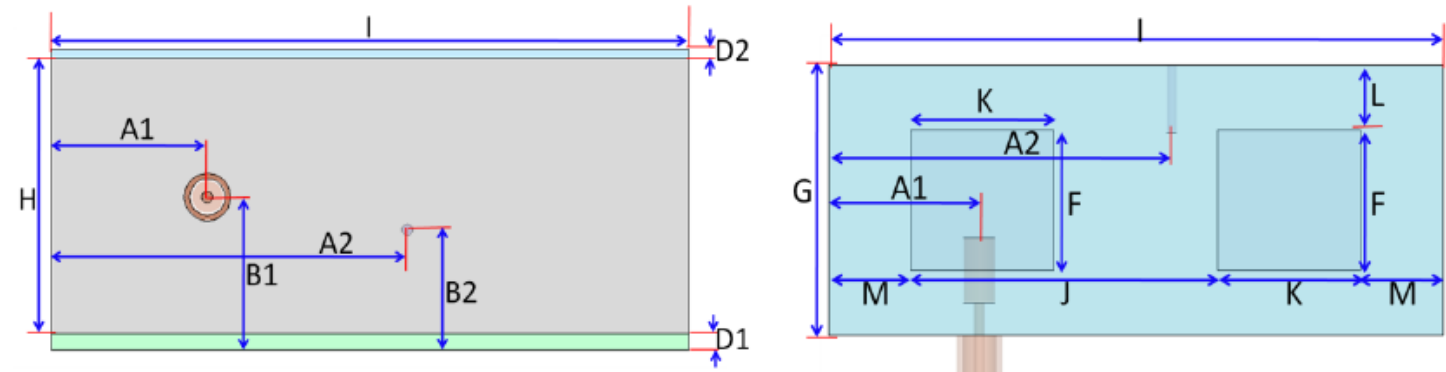

(c)

(d)

Fig. 2.7.3 Coaxial-Waveguide transition scheme ([CST 2010]). (a) Perspective view of the 3D model (b) Front view (c) Side view (d) Top view.

\begin{tabular}{|c|c|c|c|c|c|}
\hline PARAM. & DIM (mm) & PARAM. & DIM (mm) & PARAM. & DIM (mm) \\
\hline A1 & 14.2 & E1 & 3 & $\mathbf{J}$ & 30 \\
\hline $\mathbf{A 2}$ & 29 & $\mathbf{E 2}$ & 1 & $\mathbf{K}$ & 14 \\
\hline B1 & 15.524 & $\mathbf{E 3}$ & 1.02 & $\mathbf{L}=\mathbf{( G - F ) / 2}$ & 6 \\
\hline B2 & 7 & $\mathbf{E 4}$ & 3.36 & $\mathbf{M}=\mathbf{( J - K ) / 2}$ & 8 \\
\hline C1 & 1 & $\mathbf{E 5}$ & 4.36 & $\boldsymbol{\varepsilon}_{\text {rPRS }}$ & 4.4 \\
\hline C2 & 6.6 & $\mathbf{F}$ & 13 & $\boldsymbol{\varepsilon}_{\text {rHIs }}$ & 3 \\
\hline C3 & 8 & $\mathbf{G}$ & 25 & $\boldsymbol{\varepsilon}_{\text {rcoax }}$ & 2.2 \\
\hline D1 & 1.524 & $\mathbf{H}$ & 25.2 & & \\
\hline D2 & 0.8 & $\mathbf{I}$ & 60 & & \\
\hline
\end{tabular}

Table 2.7.1 Dimensions of the optimized coaxial-waveguide feeding network. 


\subsubsection{Experimental Tests and Results}

The measured $S$ parameters in the $5 \mathrm{GHz}-6 \mathrm{GHz}$ frequency range are presented in Fig. 2.7.4 for different values of bias voltage $V_{R}$. As expected, the matching band is shifted to lower frequencies as $V_{R}$ is decreased (and $C_{j}$ is increased), in concordance with the expected theoretical frequency shift observed in the leaky-mode frequency dispersion curves presented in Fig. 2.6.1. These results are also consistent with data reported in previous reconfigurable LWAs using varactors ([Sievenpiper 2002], [Sievenpiper 2003], [Hum 2005], [Mias 2007], [Sievenpiper 2005], [Costa 2008], [Weily2008], [Costa 2011]). At the fixed operation frequency of $5.6 \mathrm{GHz}$, Fig. 2.7.5 shows the simulated and measured $\mathrm{S}$ parameters as a function of $V_{R}$. As expected, the matching $\left(S_{11}\right)$ and the transmission $\left(S_{21}\right)$ coefficients are affected as $V_{R}\left(C_{j}\right)$ is varied. Simulated data predict optimum operation with $S_{11}=-23.7 \mathrm{~dB}$ and $S_{21}=-9.54 \mathrm{~dB}$ for $V_{R}=7.17 \mathrm{~V}$ (which corresponds to $C_{j}=0.15 \mathrm{pF}$, according to the datasheet (Fig. 2.5.7)).

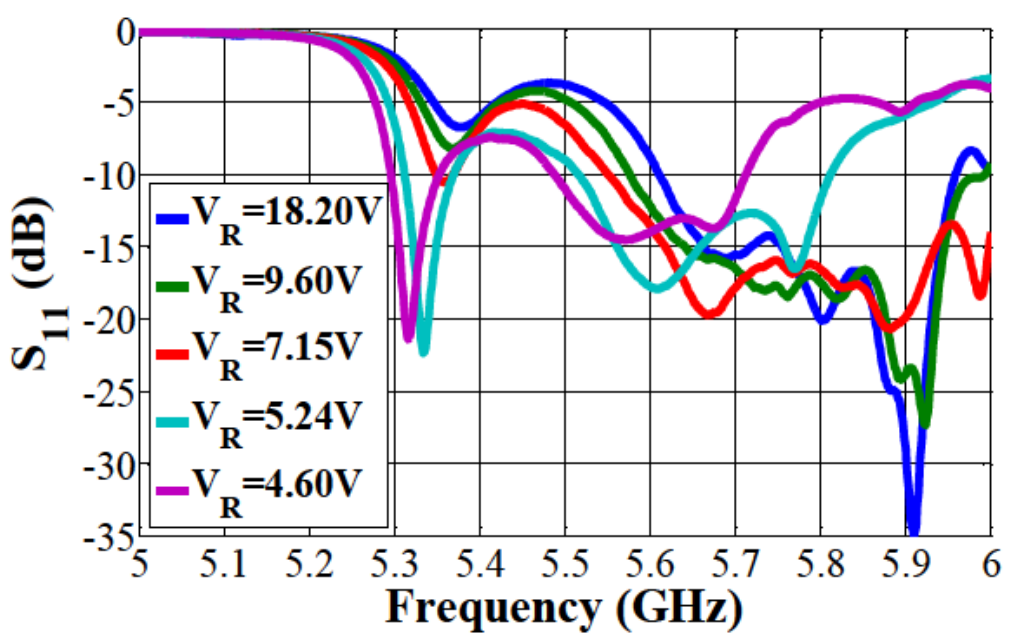

(a)

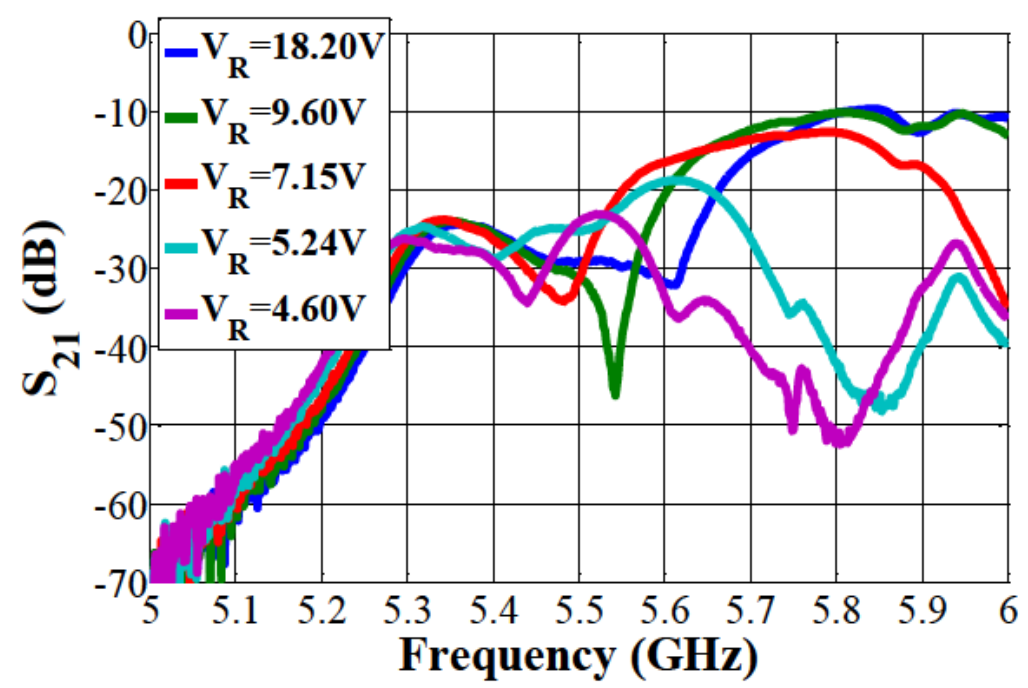

(b)

Fig. 2.7.4 Measured S parameters vs frequency for different $V_{R}$ a) $S_{11}$ b) $S_{21}$ 


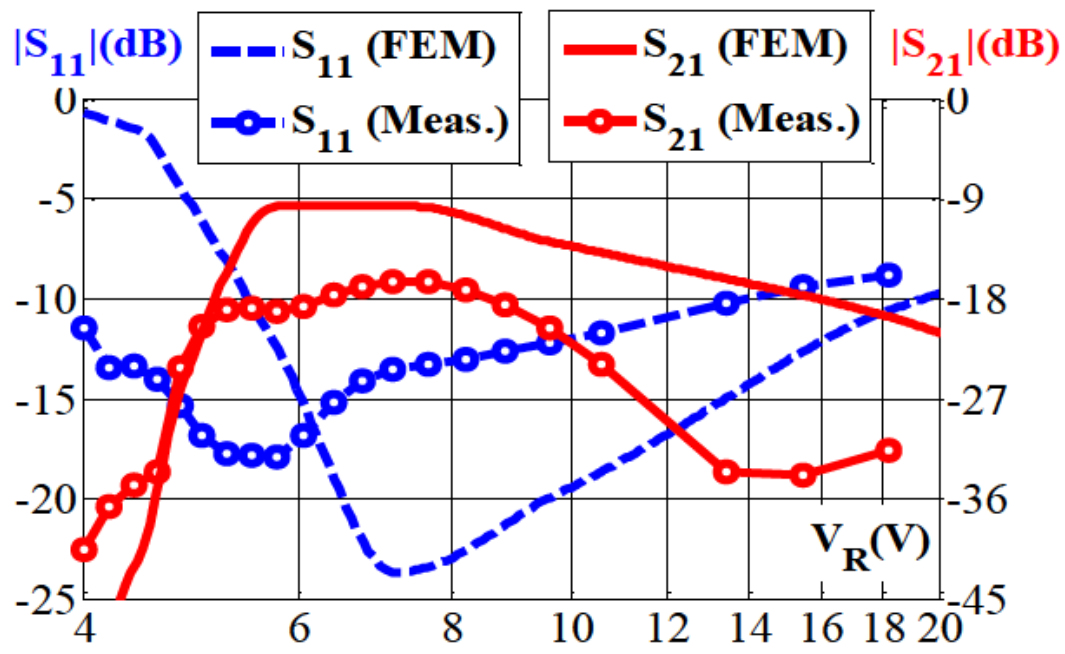

Fig. 2.7.5 Measured S parameters vs $V_{R}$ at $5.6 \mathrm{GHz}$.

As $V_{R}$ is varied from this value of $7.17 \mathrm{~V}$, the $\mathrm{TE}_{01}$ leaky-mode field distribution is strongly perturbed (as it was theoretically illustrated in Fig. 2.6.3), thus decreasing the matching between the coaxial probe and the FP cavity. It is important to note that, as previously mentioned, the coaxial-waveguide feeding dimensions were optimized for the aforementioned operating point $C_{j}=0.15 \mathrm{pF}\left(V_{R}=7.17 \mathrm{~V}\right)$. As a result, poorer matching is obtained at other operation points of the varactor's dynamic range. In particular, the mismatch increases up to $S_{I I}=-2 \mathrm{~dB}$ for $V_{R}=4.5 \mathrm{~V}$ $\left(C_{j}=0.24 \mathrm{pF}\right)$ and to $S_{11}=-10.6 \mathrm{~dB}$ for $V_{R}=18.2 \mathrm{~V}\left(C_{j}=0.06 \mathrm{pF}\right)$. Experimental data report a similar tendency for the measured $\mathrm{S}$ parameters as a function of $V_{R}$, obtaining $S_{11}=-17.9 \mathrm{~dB}$ and $S_{21}=-$ $19 \mathrm{~dB}$ at the optimum tuning point $V_{R}=5.75 \mathrm{~V}\left(C_{j}=0.19 \mathrm{pF}\right)$ and similar deterioration for other values of $V_{R}$. As can be seen, measured data is shifted to lower $V_{R}$ values with respect to simulations. This shift is attributed to the varactors' tolerance errors, as demonstrated next.

The electronic control over the LWA radiation pattern is shown in Fig. 2.7.6. Fig. 2.7.6(a) depicts the measured normalized radiation pattern at $5.6 \mathrm{GHz}$ for different values of the applied bias voltage $V_{R}$. As it can be seen, the main beam is scanned from $\theta_{R A D}=9.2^{\circ}$ for $V_{R}=18.2 \mathrm{~V}$ $\left(C_{J}=0.06 \mathrm{pF}\right)$ to $\theta_{R A D}=34.2^{\circ}$ for $V_{R}=4.5 \mathrm{~V}\left(C_{j}=0.245 \mathrm{pF}\right)$. Fig. 2.7.6(b) and Fig. 2.7.6(c) show the agreement between theory and experiments. In particular, Fig. 2.7.6(b) plots with red crosses the measured scanning response $\left(\theta_{R A D}\right.$ vs $\left.V_{R}\right)$ of the proposed reconfigurable LWA, confirming the continuous electronic scanning from $9^{\circ}$ to $34.2^{\circ}$ as $V_{R}$ is decreased from $18.2 \mathrm{~V}$ to $4.5 \mathrm{~V}$.

The theoretical leaky-mode scanning response extracted from the TRM is plotted with a continuous blue line, while FEM simulations ([CST 2010]) of a 3D model of the antenna are represented with blue circles. Very good agreement is observed between these three set of results. However, the measured scanning response is slightly shifted with respect to TRM and FEM results, showing discrepancies in $\theta_{R A D}$ that are below $\pm 2^{\circ}$. This can be attributed to the aforementioned $\pm 0.05 \mathrm{pF}$ varactors' tolerances in $C_{j}$. To demonstrate this fact, error curves 
computed by applying a shift in $C_{j}$ of $\pm 0.025 \mathrm{pF}$ and $\pm 0.05 \mathrm{pF}$ are represented with shaded zones in Fig. 2.7.6(b). As can be seen, the measured scanning response lies inside the $\pm 0.025 \mathrm{pF}$ region, below the diodes' tolerance $( \pm 0.05 \mathrm{pF})$. Fig. 2.7 .6 (c) compares the theoretical and measured leaky-mode radiation patterns for three illustrative values of $V_{R}$ covering the entire scanning range. As it was demonstrated in Fig. 2.7.6(a), the main beam direction presents higher discrepancies between theory and experiments for scanning angles close to endfire (lower $V_{R}$ values). Despite this fact, the $3 \mathrm{~dB}$ beamwidth and the secondary lobes distribution are in accordance with leaky-mode far-field patterns ([Oliner 2007]), thus confirming the leaky-wave radiation mechanism for the proposed reconfigurable antenna.

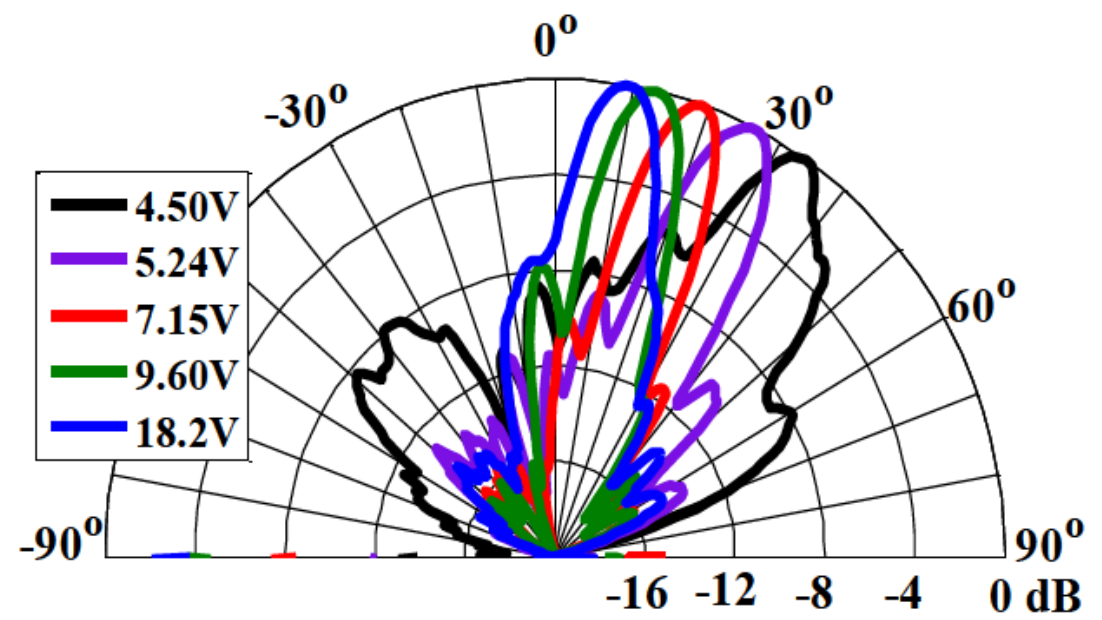

(a)

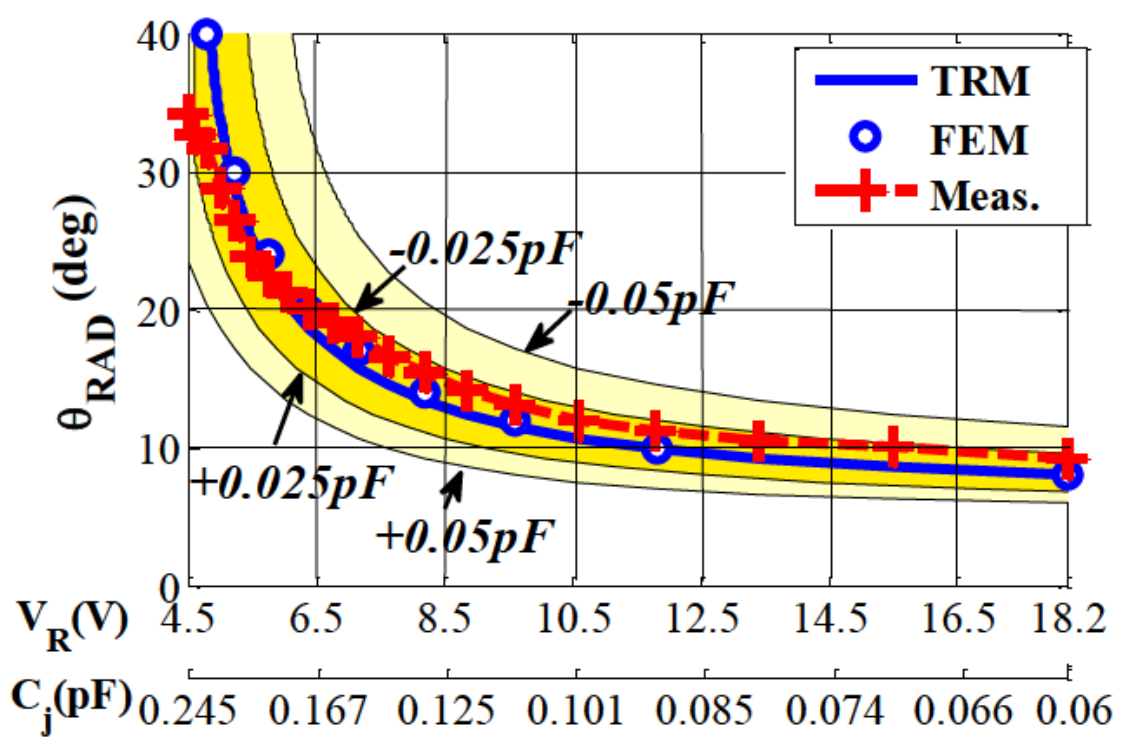

(b) 


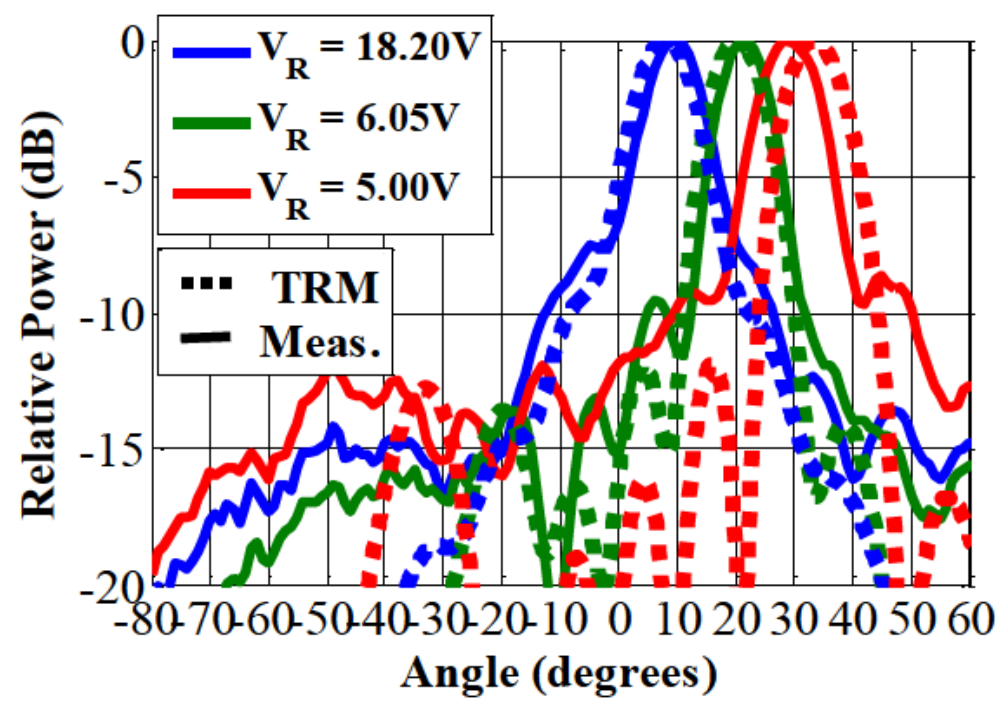

(c)

Fig. 2.7.6 Radiation response of reconfigurable LWA at 5.6 GHz vs $V_{R}$ (a) Measured radiation patterns, (b) Pointing angle scanning, (c) Comparison between measured and theoretical (TRM) radiation patterns.

\begin{tabular}{|c|c|c|c|}
\hline Case & Theory (TRM) & Experiments & Error \\
$\boldsymbol{V}_{R}(\mathbf{V})$ & $\boldsymbol{\theta}_{R A D}$ & $\boldsymbol{\theta}_{R A D}$ & $\Sigma \theta_{R A D}$ \\
\hline $\mathbf{5 . 0}$ & $34.1^{\circ}$ & $28.8^{\circ}$ & $-5.3^{\circ}$ \\
\hline $\mathbf{6 . 0}$ & $20.5^{\circ}$ & $21.2^{\circ}$ & $+0.7^{\circ}$ \\
\hline $\mathbf{1 8 . 0}$ & $8^{\circ}$ & $9.2^{\circ}$ & $+1.2^{\circ}$ \\
\hline
\end{tabular}

Table 2.7.2 Bias voltage and pointing angle for three operating points of the reconfigurable 1D FP LWA.

Table 2.7.2 summarizes the pointing angle $\theta_{R A D}$ obtained for three operation points inside the scanning region. As it is shown, maximum error is obtained at lower voltages that correspond to larger scan angles $\left(V_{R}=5 \mathrm{~V}, \Sigma \theta_{R A D}=-5.3^{\circ}\right)$. It is worthy to note that the scanning response predicted by the lossless TRM is in agreement with the response of the lossy simulated model and the experimental prototype, despite the losses created by the varactors series resistance and materials. This confirms that thermal losses do not have a strong influence on the leaky-mode phase constant, and the proposed lossless TRM is useful for the design of this antenna. 


\subsection{Efficiency Analysis}

The efficiency of an antenna basically defines the ratio between energy radiated and energy lost by dissipative losses of components (substrate, metals, electronic devices) and mismatching. Efficiency must be taken into account for any professional antenna design process considered, and especially in case of reconfigurable antennas, where dissipative losses of substrates or incorporated electronic devices (e.g., diodes, capacitances, resistances, inductances, etc.) may become very high, depending on the operation point of the antenna.

In this subsection, the efficiency of the 1D FP LWA is further studied in order to analyze the effect of mismatching, substrate materials and varactor losses on the maximum gain of the antenna, as a function of the operating point.

Fig. 2.8.1 summarizes the experimental performance of the reconfigurable LWA in terms of gain and efficiency. Fig. 2.8.1(a) shows the variation of the gain as a function of $V_{R}$, reporting maximum measured gain of $G=12.95 \mathrm{dBi}$ for $V_{R}=10.6 \mathrm{~V}\left(C_{j}=0.1 \mathrm{pF}\right.$, measured $\left.\theta_{R A D}=12^{\circ}\right)$, while simulations predict maximum $G=12.6 \mathrm{dBi}$ for $V_{R}=9.6 \mathrm{~V}\left(C_{j}=0.11 \mathrm{pF}\right.$, simulated $\left.\theta_{R A D}=11^{\circ}\right)$. Moreover, as $V_{R}$ is decreased from this optimum point, a significant drop in the gain is observed. The minimum value of gain measured is $G=-3.55 \mathrm{dBi}$ for $V_{R}=4.2 \mathrm{~V}\left(C_{j}=0.26 \mathrm{pF}\right)$, which corresponds to a measured angle close to $\theta_{R A D}=35^{\circ}$. Similar behavior is obtained with simulations, thus making it difficult to obtain large scanning angles closer to endfire. As it was previously explained, this fact is caused by higher mismatch (see Fig. 2.7.5) and by the fall of the leakage rate (see Fig. 2.6.1(c)) that take place at high values of $C_{j}$ (lower $V_{R}$ ), due to the associated PMC resonance of the HIS for high $\theta_{R A D}$ (see Fig. 2.6.3). However, the LWA shows a stable gain above $G=10 \mathrm{dBi}$ for $V_{R}>6 \mathrm{~V}$, which corresponds to scanning angles below $\theta_{R A D}=25^{\circ}$. As also plotted in Fig. 2.8.1(a), this gain translates into a total efficiency $\eta_{T O T}=G / D>50 \%$, reaching $\eta_{T O T}=75 \%$ for $V_{R}=10-14 \mathrm{~V}\left(\theta_{R A D}=13^{\circ}-10^{\circ}\right)$. Measured and simulated total efficiency $\left(\eta_{T O T}\right)$ in Fig. 2.8.1(a) are in concordance, observing $\eta_{T O T}>60 \%$ in the range $V_{R}=8-20 \mathrm{~V}$ $\left(\theta_{R A D}=16^{\circ}-10^{\circ}\right)$, whereas $\eta_{T O T}$ tends to $3 \%$ for $V_{R} \leq 5 \mathrm{~V}\left(\theta_{R A D} \geq 30^{\circ}\right)$. To understand the gain and efficiency response of the LWA, Fig. 2.8.1(b) separates $\eta_{\text {Tот }}$ into its different constitutive efficiencies ([Balanis 2005, Chapter 2], [Gómez 2007]):

$$
\eta_{T O T}=\eta_{M I S} \cdot \eta_{R A D} \cdot \eta_{D I E} \cdot \eta_{V A R}
$$

where the mismatch efficiency is computed from the measured input reflection $\left(\eta_{M I S}=1-\right.$ $\left|S_{11}\right|^{2}$ ) ([Balanis 2005, Chapter 2]), the leaky-mode radiation efficiency is estimated from the theoretical leakage rate $\left(\eta_{R A D}=1-e^{-2 \alpha_{y} L_{A}}\right)$ ([Gómez 2007]), and the ohmic efficiency has been attributed to two contributions: one part due to the dielectric losses and another term due to 
dissipation in the varactors series resistance: $\eta_{\Omega}=\eta_{\text {DIE }} \cdot \eta_{\text {VAR }}$ ([Balanis 2005, Chapter 2]). Since the separated ohmic efficiencies (due to dielectrics and varactors) are difficult to compute from experiments ([Balanis 2005, Chapter 2]), they have been estimated from full-wave simulation ([CST 2010]). Eq.(2.8.1) gives, as a first order approximation, a good insight into the origin of the gain drop for large scan angles. Particularly, it can be seen in Fig. 2.8.1(b) that the mismatch efficiency $\eta_{M I S}$ is quite high (over $85 \%$ ) for the entire dynamic range.

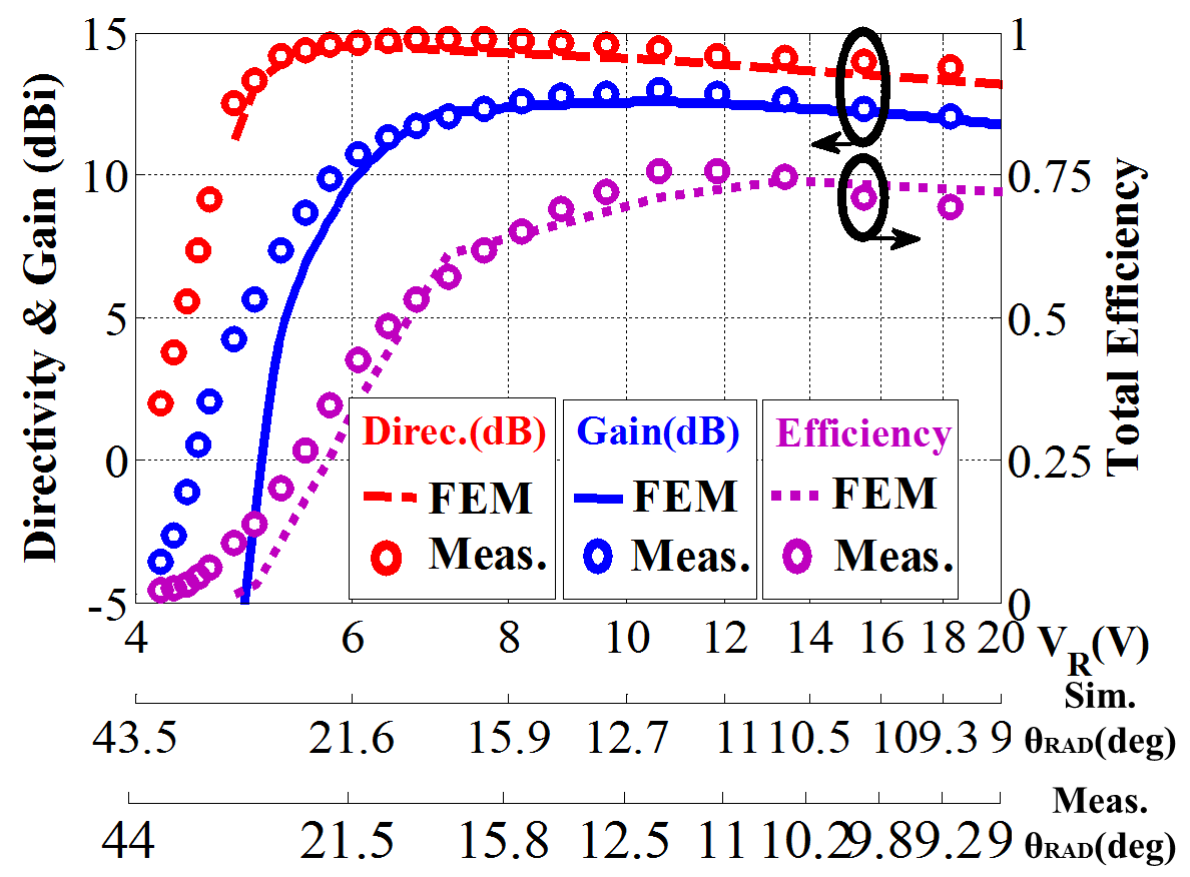

(a)

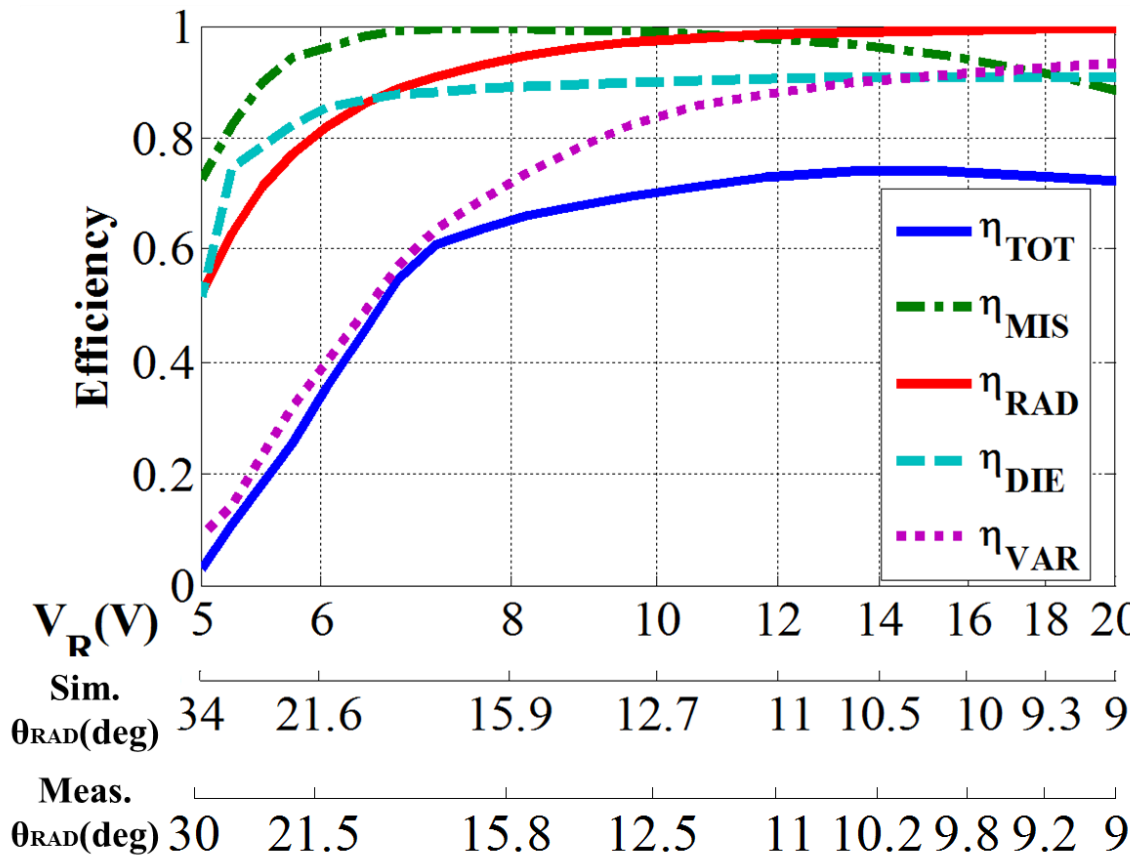

(b) 

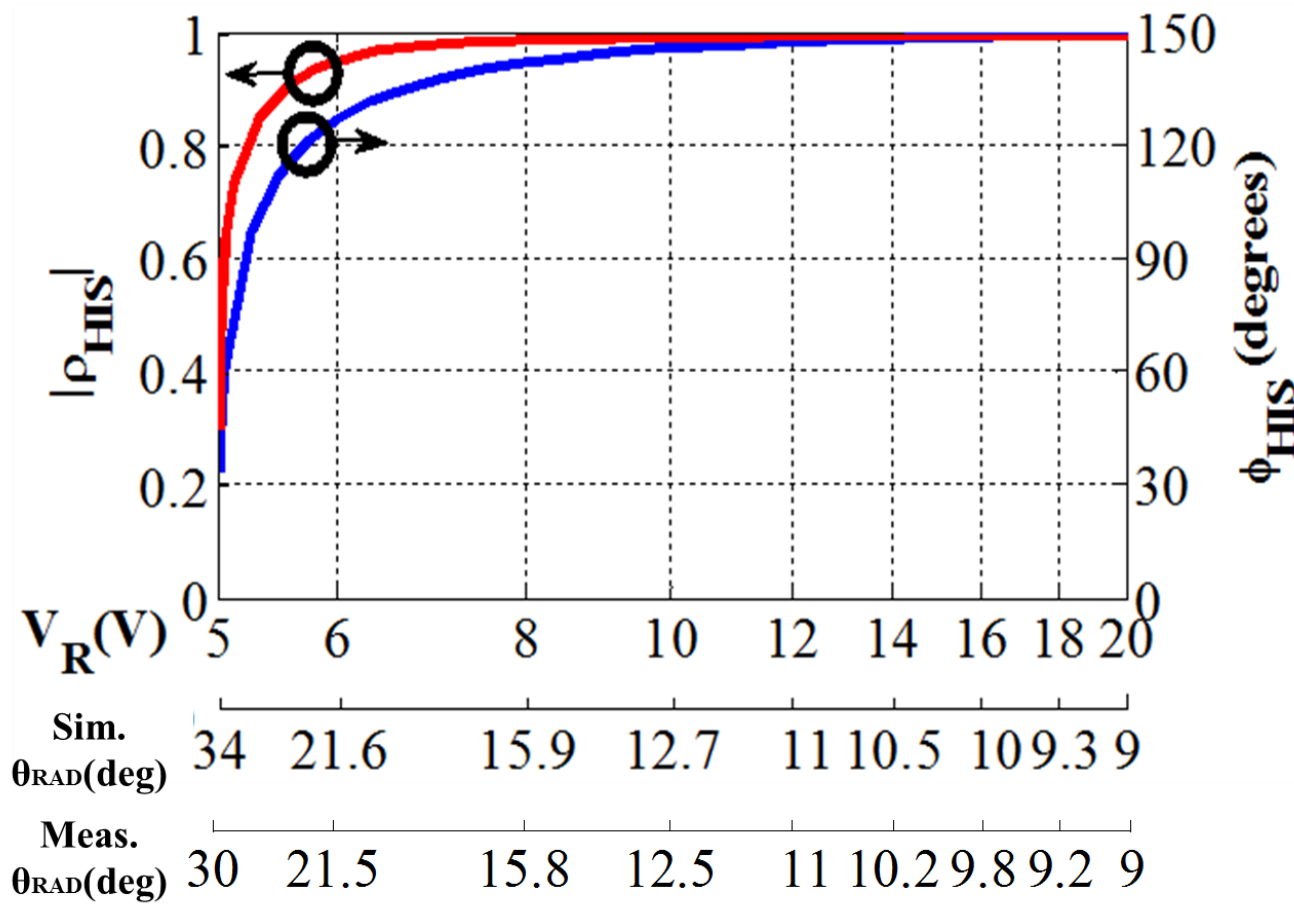

(c)

Fig. 2.8.1 Results at 5.6GHz vs $V_{R}$ (a) Measured gain, directivity and total efficiency, (b) Estimated efficiencies and (c) Simulated reflection coefficient (magnitude and phase) given by the tunable HIS (taken into account losses) as a function of the operating point of the antenna (given by simulations).

On the other hand, the leaky-mode radiation efficiency $\eta_{R A D}$ strongly decreases from $100 \%$ at $V_{R}=20 \mathrm{~V}$ (simulated $\theta_{R A D}=8^{\circ}$ and measured $\theta_{R A D}=9^{\circ}$ ) to $52 \%$ at $V_{R}=5 \mathrm{~V}$ (simulated $\theta_{R A D}=34.2^{\circ}$ and measured $\theta_{R A D}=30^{\circ}$ ), being consistent with the drop of the leakage rate predicted in Fig. 2.6.1(c) for high values of $C_{j}$ (i.e., high values of $\theta_{R A D}$ ). The dielectric efficiency results predict a fall from $\eta_{D I E}=90 \%$ at $\theta_{R A D}=9^{\circ}\left(V_{R}=20 \mathrm{~V}\right)$ to $\eta_{D I E}=50 \%$ at $\theta_{R A D}=34^{\circ}\left(V_{R}=5 \mathrm{~V}\right)$, which is caused by the electric field concentration at the HIS substrate when operates close to the PMC regime (see Fig. 2.6.3 for $C_{j}=0.23 \mathrm{pF}$ ). Due to the same high concentration of the modal fields, an increase in the current density flowing across the diodes occurs at this PMC operational point, raising the heat dissipation at their series resistance. As a result, the varactors' ohmic efficiency $\eta_{V A R}$ strongly drops for scanning angles tending to endfire, observing a decrease from $\eta_{V A R}=$ $94 \%$ at $\theta_{R A D}=9^{\circ}\left(V_{R}=20 \mathrm{~V}\right)$ to $\eta_{V A R}=9 \%$ at $\theta_{R A D}=30^{\circ}\left(V_{R}=5 \mathrm{~V}\right)$.

This increase of thermal losses in the HIS as the antenna scans is demonstrated in Fig. 2.8.1(c). The reflection coefficient of the HIS has been performed by full-wave FEM ([CST 2010]) for each operating point $\left(C_{j}, \theta_{R A D}\right)$ of the designed antenna. As it can be observed, the HIS absorption increases as the LWA scans towards endfire, and for $\theta_{R A D}=34^{\circ}$ there is an abrupt drop to $\left|\rho_{H I S}\right|=0.3$. This is related with the strong change of the HIS phase, which operates close to 
the PMC resonance. A HIS reflection phase of $\phi_{H I S}=30^{\circ}$ is estimated when antenna is pointing close to $\theta_{R A D}=30^{\circ}$, in accordance with the lossless HIS response shown in Fig. 2.5.11(b) for $C_{j}=0.23 \mathrm{pF}$. This result agrees with other published works that report significant increase in the HIS ohmic losses when operating close to the PMC resonance, and which has even been widely applied to create planar microwave absorbers ([Engheta 2002], [Tretyakov 2003-II], [Costa 2010]). Therefore, it can be concluded that the gain drop for large scan angles is unavoidable and it is due to two main reasons: the natural decrease in the leaky-mode radiation efficiency as the scanning angle increases, and the increase of the varactors resistance. This latter effect could have also been predicted by the proposed TEN model, by introducing the varactors' series resistance in the tunable HIS equivalent admittance, and the losses tangent in the substrate equivalent transmission line sections, as done in [Engheta 2002], [Tretyakov 2003-I], and [Costa 2010]. The characterization of lossy FSS by the pole-zero method would require additional modifications in order to account for the real part of the admittances, which would be no longer assumed purely imaginary due to ohmic losses. This analysis has been left as a possible research future line. 


\subsection{Analysis of the Scanning Pattern Bandwidth}

\subsubsection{Introduction}

Frequency scanning is an inherent property of LWAs due to their intrinsic highly dispersive nature ([Oliner 2007]). This can be a desired feature for some applications such as frequencymodulated continuous-wave (FMCW) radars ([Ettorre 2008]). On the contrary, frequency beam squint reduces the bandwidth for point-to-point telecom radio links, since the gain at the desired pointing angle can rapidly drop out from the design frequency as sketched in Fig. 2.9.1. For this reason, much interest is given nowadays to the design of scanning LWAs with reduced beam squint ([Hong 2003], [Neto 2005], [Antoniades 2008], [Sievenpiper 2011]). The definition of the practical usable bandwidth of a LWA must therefore consider not only the input matching bandwidth but also the scanned pattern bandwidth ([Balanis 2005]). This is particularly intriguing for electronically-reconfigurable LWAs ([Lim 2004-I, II], [Archbold 2010], [Sievenpiper 2005], etc.), which operate at a fixed frequency and dynamically change their scanning response by means of an external control signal.

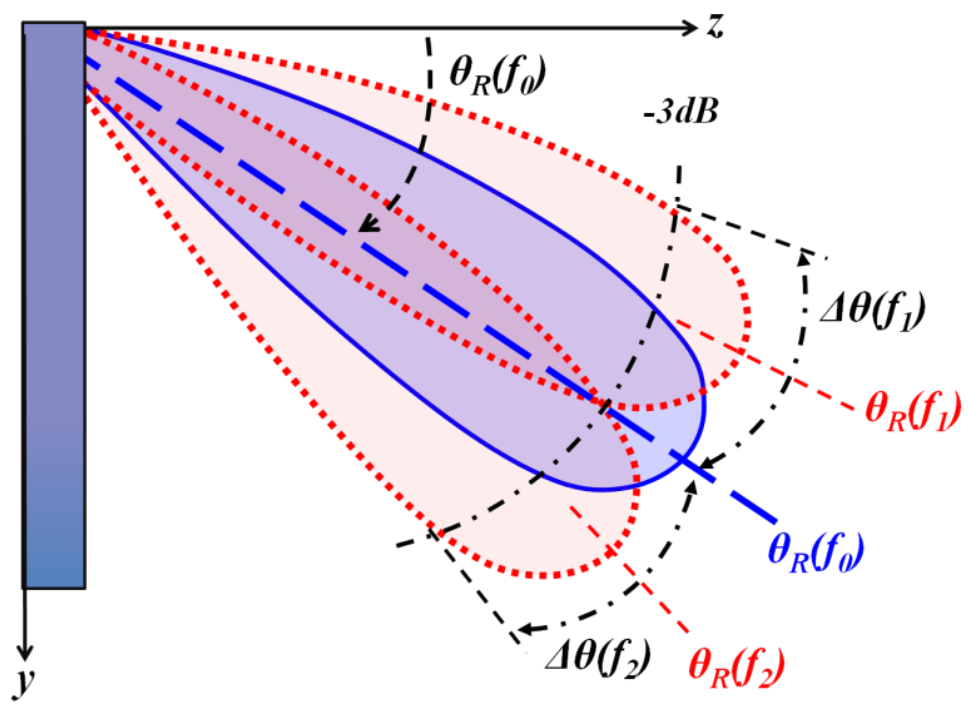

Fig. 2.9.1 Pattern bandwidth definition from the frequency scanning of a LWA

In this section we propose a closed-form expression to define and evaluate the maximum usable pattern bandwidth of electronically-steerable LWAs. Subsection 2.9.2 describes the theoretical formulation, which is derived from the frequency-dispersion response of the electronicallycontrolled leaky mode. This general theory is applied in Subsection 2.9.3 to the electronically reconfigurable scanning 1D FP LWA previously proposed. Full-wave simulated results are used for validation. 


\subsubsection{Scanning PBW Theory}

The pattern bandwidth of an antenna is usually defined as the frequency range $\left[f_{1}, f_{2}\right]$ over which the gain at a fixed observation direction $\theta_{0}$ falls $3 \mathrm{~dB}$ w.r.t. the gain at the design frequency $f_{0}$ $\left(f_{1}<f_{0}<f_{2}\right)$ ([Balanis 2005]). For the case of a LWA, the beam is scanned as frequency is varied as shown in Fig. 2.9.1, so that the condition for the scanned pattern bandwidth (SPBW) can be approximately expressed as:

$$
\begin{aligned}
& \theta_{R}\left(f_{1}\right)+\frac{\Delta \theta\left(f_{1}\right)}{2}=\theta_{R}\left(f_{0}\right) \\
& \theta_{R}\left(f_{2}\right)-\frac{\Delta \theta\left(f_{2}\right)}{2}=\theta_{R}\left(f_{0}\right)
\end{aligned}
$$

where $\theta_{R}(f)$ is the main beam scanning angle and $\Delta \theta(f)$ is the half-power beamwidth at a certain frequency. The antenna frequency squint, also called scanning ratio $(S R)$, is given in $\mathrm{deg} / \mathrm{Hz}$ and is defined as the first derivative of the frequency beam scanning response:

$$
S R(f)=\frac{d \theta_{R}(f)}{d f}
$$

Subtracting Eq.(2.9.1) and Eq.(2.9.2) and assuming small difference between $f_{2}$ and $f_{1}$ (which is true for narrow band radiators as usually happens with LWAs), we can introduce Eq.(2.9.3) and obtain the following expression:

$$
\theta_{R}\left(f_{2}\right)-\theta_{R}\left(f_{1}\right) \approx S R\left(f_{0}\right) \cdot\left[f_{2}-f_{1}\right] \approx \frac{1}{2}\left[\Delta \theta\left(f_{2}\right)+\Delta \theta\left(f_{1}\right)\right]
$$

The scanned pattern bandwidth $\left(\mathrm{SPBW}=f_{2}-f_{l}\right)$ can thus be approximated as:

$$
S P B W \approx \frac{1}{2} \frac{\Delta \theta\left(f_{2}\right)+\Delta \theta\left(f_{1}\right)}{S R\left(f_{0}\right)} \approx \frac{\Delta \theta\left(f_{0}\right)}{S R\left(f_{0}\right)}
$$

where it has also been assumed that the beamwidth keeps constant in the studied frequency range $\Delta \theta\left(f_{2}\right)=\Delta \theta\left(f_{1}\right)=\Delta \theta\left(f_{0}\right)$. For the case of LWAs, the frequency dispersion response of the leaky-mode complex propagation constant gives all the information for the functions involved in Eq.(2.9.5) ([Oliner 2007]):

$$
\begin{aligned}
& k(f)=\beta(f)-j \alpha(f) \\
& \sin \left(\theta_{R A D}\right) \approx \beta_{y} / k_{0} \\
& \Delta \theta(f) \approx \frac{k}{L_{A} / \lambda_{0} \cdot \cos \theta_{R A D}(f)}
\end{aligned}
$$


where $L_{A}$ is the LWA aperture length and $k$ a constant which depends on the antenna aperture field distribution ([Oliner 2007]); for this case $k=0.91$ considering natural exponential leaky wave illumination and assuming 90\% of radiation. Introducing Eq.(2.9.8) in Eq.(2.9.5), we obtain the next approximation to the SPBW:

$$
S P B W \approx \frac{k}{S R\left(f_{0}\right) \cdot L_{A} / \lambda_{0} \cdot \cos \theta_{R}(f)}
$$

The scanning pattern bandwidth is inversely proportional to three frequency-dependent functions according to Eq.(2.9.9). First, a high scanning ratio (Eq.(2.9.3)) makes the LWA scan more rapidly and thus decrease the resulting SPBW. Second, longer LWAs produce a narrower beam (Eq.(2.9.8)) and thus decrease the resulting SPBW. Finally, the scanning angle $\theta_{R}(f)$ also plays an important role, since LWAs scanning closer to broadside $\left(\theta_{R}=0^{\circ}, \cos \theta_{R}=1\right)$ have a larger effective aperture and directivity, and thus provide reduced SPBW than if scanned towards endfire, where directivity is usually lower.

These three effects must be taken into account when evaluating the practical bandwidth of an electronically reconfigurable LWA. Certainly, this type of reconfigurable antennas steer their pointing direction at different angles depending on an external control signal $\left(V_{R}\right)$. Therefore, the SPBW is dynamic, and must be studied for all the operation states. Apart from the obvious variation of the scanning angle $\theta_{R}$, the dispersive response of the antenna also varies as a function of the control signal. Thus, the scanning ratio and the resulting bandwidth will be dependent on the scanning state in which the antenna is operating. This will be illustrated in the following section by analyzing the SPBW of a practical reconfigurable LWA, which will also be used to validate the derived theoretical expression for the SPBW (Eq.(2.9.9)). 


\subsubsection{Analysis Example on the Electronic-Steering 1D FP LWA}

The method proposed can be applied to many types of electronically tunable LWAs with continuous scanning at a fixed operation frequency $f_{0}$, for instance [Lim 2004-I, II], [Archbold 2010], [Sievenpiper 2005]. According to Eq.(2.2.4), the leaky-mode normalized phase constant $\beta / k_{0}$ must be properly modified to cover a wide range of scanning angles $\theta_{R}$ at the design frequency $f_{0}$. In this section, we study the SPBW for the reconfigurable Fabry-Pérot (FP) LWA proposed in this chapter. Previous works of other authors have treated the bandwidth of FP LWAs which are normally narrow-band ([Boutayeb 2007], [Feresidis 2006], [Hosseini 2011], [Lovat 2006-II], [Mateo-Segura 2014], [Yuehe 2012], [Zhao 2005]). However, these studies were focused on fixed (not reconfigurable) two-dimensional (2D) FP LWAs radiating at broadside. However, the study proposed here is totally different since we deal with reconfigurable scanning 1D LWAs.

For the reconfigurable beam-scanning 1D FP LWA designed in this chapter, Fig. 2.9.2(a) shows the leaky-mode dispersion curves as a function of frequency Eq.(2.9.6) for different control voltages $\left(V_{R}\right)$. From these dispersion curves and applying equations Eq.(2.9.7) and Eq.(2.9.3), the scanning angle $\theta_{R}(f)$ and the scanning ratio $S R(f)$ responses of the antenna are respectively obtained and plotted in Fig. 2.9.2(b). Note that Eq.(2.9.3) has been readily obtained as a numerical finite difference from the dispersion curves. It is evident that the control signal $V_{R}$ modifies the frequency dispersion response of the leaky mode, increasing the cutoff frequency as the voltage $V_{R}$ is increased.

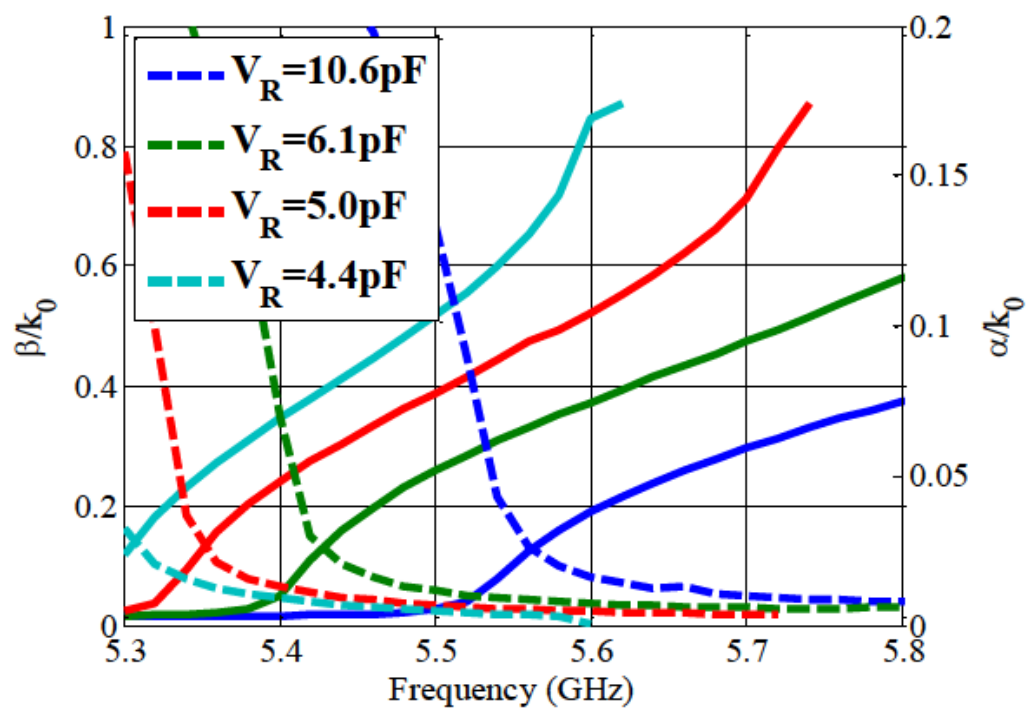

(a) 


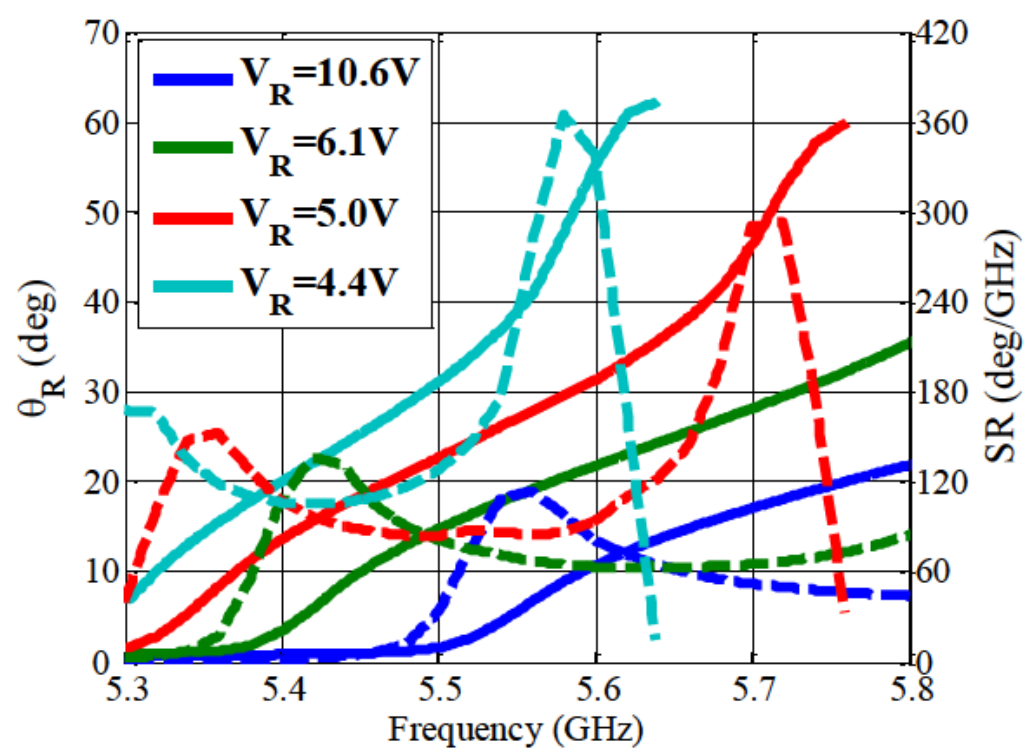

(b)

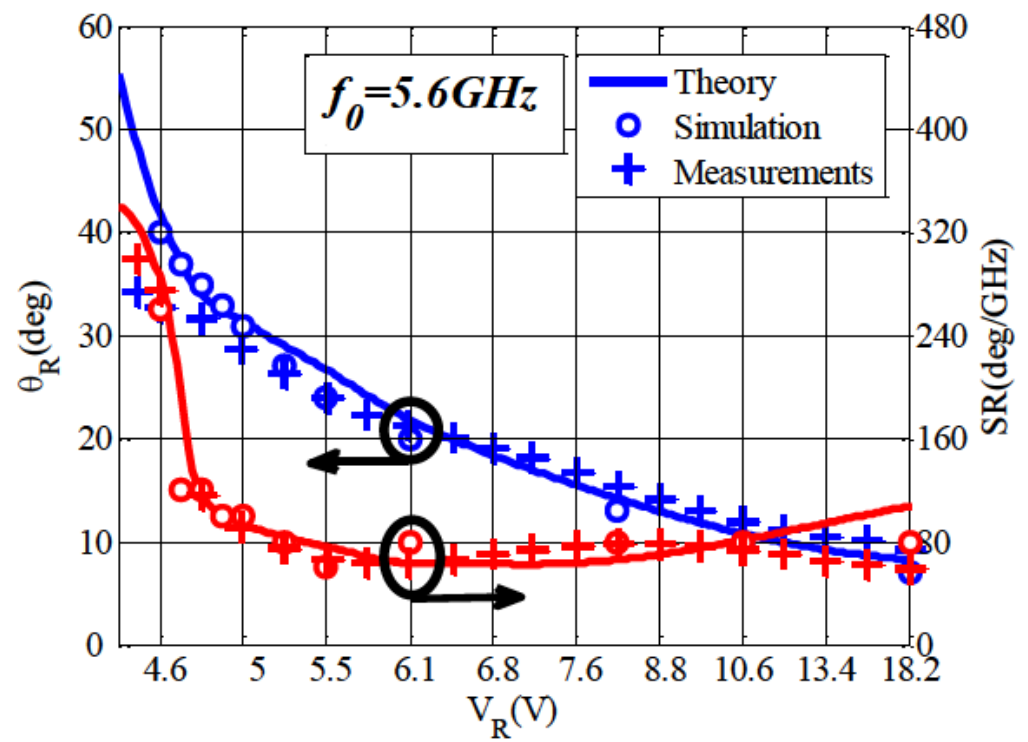

(c)

Fig. 2.9.2 (a) Normalized leaky-mode dispersion curves $\left(\beta / k_{0}\right.$ and $\left.\alpha / k_{0}\right)$ obtained from TEN for the half-space electronic scanning 1D FP LWA and (b) Scanning angle $\left(\theta_{R}\right)$ and scanning ratio (SR) as a function of frequency for different $V_{R}$ cases. (c) Scanning response and frequency scanning ratio as a function of $V_{R}$ at the fixed design frequency $f_{0}=5.6 \mathrm{GHz}$.

Again, it can be observed that if the operating frequency is fixed to $f_{0}=5.6 \mathrm{GHz}$, this mechanism provides a continuous electronic control over the scanning angle, as observed in Fig. 2.9.2(c), where theoretical, simulated and measured scanning angle and scanning ratio is represented as a function of $V_{R}$. The dependence of the scanning ratio $S R$ (Eq.(2.9.3)) on the antenna operating state can be attributed to the non-linear dispersion of the $\theta_{R}(f)$ curves, which experiment an increase of their slope close to the lower cutoff frequency and especially to the upper stopband region. This type of response is common to structures loaded with High Impedance Surfaces 
(HIS) ([Sievenpiper 2003, 2005], [García-Vigueras 2012-II]). As a result, the scanning ratio $S R$ for the design frequency of $f_{0}=5.6 \mathrm{GHz}$ shows a dependence with the control signal, as shown by the red curve in Fig. 2.9.2(c). Particularly, when the antenna is scanning at angles around $\theta_{R}=20^{\circ}\left(V_{R}=6.4 \mathrm{~V}\right)$ it is observed a soft variation of the theoretical scanning ratio around $S R=70^{\circ} / \mathrm{GHz}$. However, when the antenna is reconfigured to operate at higher angles around $\theta_{R}=40^{\circ}\left(V_{R}=4.6 \mathrm{~V}\right)$, it is observed an increase in the frequency angular sensitivity up to $S R=260^{\circ} / \mathrm{GHz}$. A smaller increase of the $S R$ is observed in theory for lower scanning angles $\left(S R=100^{\circ} / \mathrm{GHz}\right.$ for $\theta_{R}=8^{\circ}$ with $\left.V_{R}=18.2 \mathrm{~V}\right)$.

These theoretical curves shown in Fig. 2.9.2 are the same obtained from the TRM in previous sections. Simulated and measured results presented in Fig. 2.9.2(c) validate the leaky-mode dispersive response as a function of the control signal $\left(V_{R}\right)$. Remembering from previous sections, the prototype presented an aperture length $L_{A}=5.2 \lambda_{0}(280 \mathrm{~mm})$ and an approximated usable scanning range from $\theta_{R}=+9^{\circ}$ to $\theta_{R}=+30^{\circ}$, limited by the effect of losses and assuming a minimum realized gain $\mathrm{G} \geq 5 \mathrm{dBi}$.

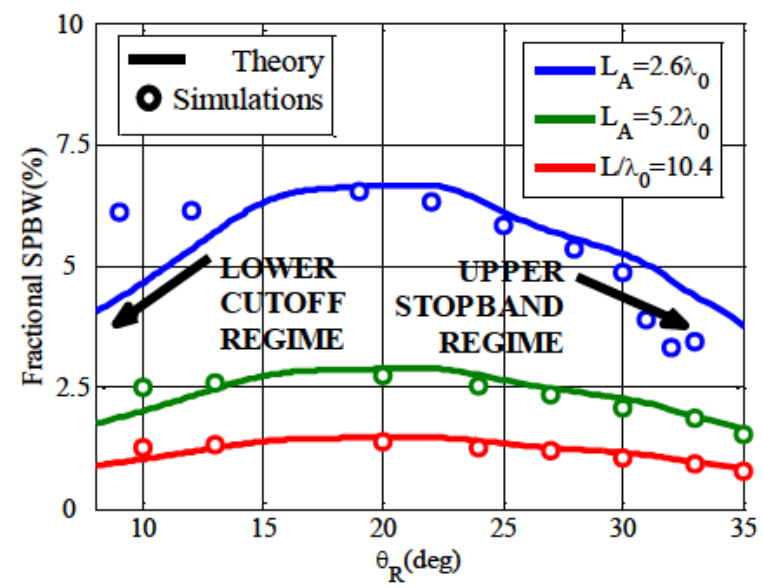

Fig. 2.9.3 Fractional pattern bandwidth (FPBW) as a function of scanning angle $\left(\theta_{R}\right)$ and antenna aperture length $\left(L_{A}\right)$ (theoretical lossless case).

Once the dispersion curves have been obtained for different operating states of the reconfigurable antenna, the SPBW can be directly estimated from Eq.(2.9.9) and for different scanning angles. Fig. 2.9.3 shows the relative Fractional SPBW (FSPBW=SPBW $/ f_{0}$ ) at $f_{0}=5.6 \mathrm{GHz}$ as a function of the antenna operating scanning angle $\left(\theta_{R}\right)$ and for three different antenna aperture lengths $\left(L_{A}\right)$. For a fixed antenna length, it is observed that the SPBW depends on the scanning state, observing higher bandwidth for intermediate scanning angles $\left(\theta_{R} \approx 20^{\circ}\right)$. As previously discussed, this is mainly due to the increase of the $S R$ for scanning angles close to broadside $\left(\theta_{R}<10^{\circ}\right.$ where the leaky-mode lower band cutoff occurs) and angles tending to endfire with $\theta_{R}>30^{\circ}$ (where the HIS upper stop band arises). This is an important effect which limits the overall BW of a reconfigurable LWA, and which has not been reported until now. For 
instance, the case of $L_{A}=5.2 \lambda_{0}$ shows a theoretical SPBW $=2.88 \%$ when scanned at $\theta_{R}=20^{\circ}$, which is reduced to SPBW $=2.02 \%$ for $\theta_{R}=10^{\circ}$ and to SPBW $=2.26 \%$ for $\theta_{R}=30^{\circ}$.

In addition, it is also appreciated the strong influence of $L_{A}$, evidencing an inverse relationship with the SPBW as stated by Eq.(2.9.9). This is reasonable since longer LWAs provide reduced half-power beamwidth $\Delta \theta$ (Eq.(2.9.8)), which results in a stronger effect of the frequency beam steering and thus lower SPBW. This can be clearly observed if we fix $\theta_{R}=20^{\circ}$ in Fig. 2.9.3, observing a theoretical maximum bandwidth of $S P B W=6.65 \%$ for $L_{A}=2.6 \lambda_{0}$, whereas a lower bandwidth of $F S P B W=1.66 \%$ is achieved for $L_{A}=10.4 \lambda_{0}$.

Theoretical results of Fig. 2.9.3 are compared with full-wave simulations (plotted with circles), obtained for the ideal (lossless and perfectly matched) reconfigurable antenna prototype. Good agreement with theoretical predictions is obtained despite the simplicity of our formula, which only takes into account the dispersion of the antenna, neglecting directivity or beamwidth variability. In addition, the derived expressions are more accurate for longer antennas which are narrowband and present lower beamwidths, as discussed in the approximations made to derive the formulation (Eq.(2.9.4)-Eq.(2.9.5)). This is observed for the longer studied case $\left(L_{A}=10.4 \lambda_{0}\right)$, which simulations almost fit the theoretical curve.

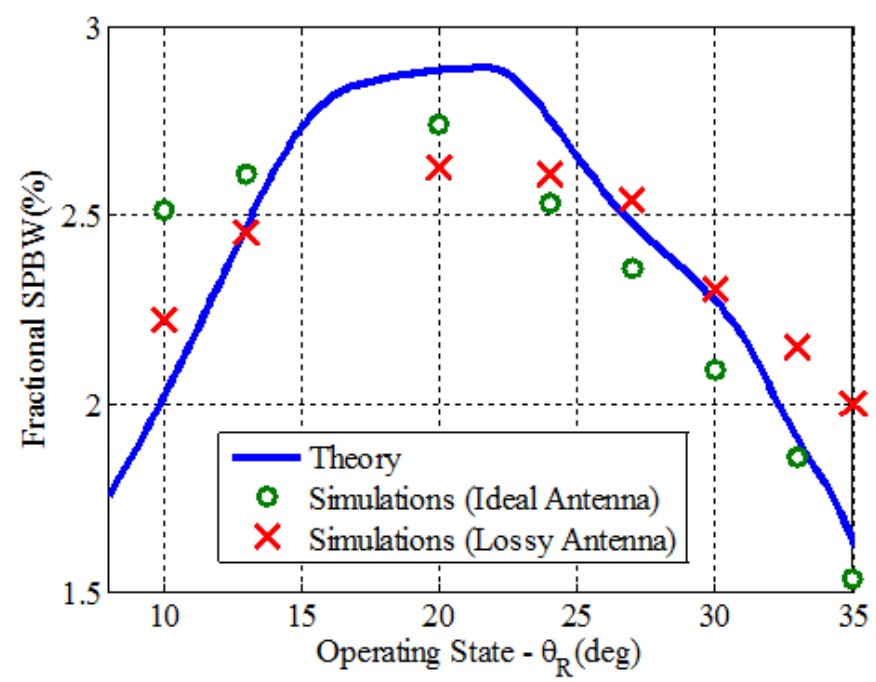

Fig. 2.9.4 Comparison of Fractional PBW as a function of operating scanning point $\left(\theta_{R}\right)$ obtained from theory, ideal lossless simulations and lossy simulations.

All previous results have treated with ideal lossless antennas and neglected mismatching. However, in the real world these effects must be considered to obtain the real usable bandwidth. Fig. 2.9.4 compares the FSPBW presented in Fig. 2.9.3 for an ideal antenna with $L_{A}=5.2 \lambda_{0}$, with the FSPBW obtained from simulations of the final prototype, which have taken into account losses and a real excitation source. From this graph it is observed how our model still remains in 
good agreement. Table 2.9.1 compares theory, lossless simulations and lossy simulations of the final prototype for three representative antenna operating states $\left(\theta_{R}=10^{\circ}, \theta_{R}=20^{\circ}, \theta_{R}=30^{\circ}\right)$. It is demonstrated good SPBW theoretical prediction with absolute errors lower than $0.5 \%$, whereas lower error is expected for longer antennas, given the results presented in Fig. 2.9.3. In addition, it is demonstrated that the maximum usable bandwidth for this antenna is approximately $2.22 \%$, given by the most restrictive operating states (in terms of bandwidth) which are observed in this case $\left(\theta_{R}=10^{\circ}, 30^{\circ}\right)$, which are located at the extremes of the considered scanning range, as expected.

\begin{tabular}{cccccc}
\hline $\boldsymbol{\theta}_{R A D}$ & $\begin{array}{c}\text { FPBW } \\
\text { (Theory) }\end{array}$ & $\begin{array}{c}\text { FPBW } \\
\text { (Ideal) }\end{array}$ & $\begin{array}{c}\text { FPBW } \\
\text { (Lossy) }\end{array}$ & $\begin{array}{c}\text { Error } \\
\text { (Ideal) }\end{array}$ & $\begin{array}{c}\text { Error } \\
\text { (Lossy) }\end{array}$ \\
\hline $\mathbf{1 0}^{\circ}$ & $2.02 \%$ & $2.51 \%$ & $2.22 \%$ & $0.49 \%$ & $0.20 \%$ \\
$\mathbf{2 0}^{\circ}$ & $2.88 \%$ & $2.73 \%$ & $2.62 \%$ & $0.15 \%$ & $0.26 \%$ \\
$\mathbf{3 0}^{\circ}$ & $2.26 \%$ & $2.08 \%$ & $2.30 \%$ & $0.18 \%$ & $0.04 \%$ \\
\hline
\end{tabular}

Table 2.9.1 Calculated Scanning Ratio for three scanning angles $\left(\theta_{R A D}\right)$ of the reconfigurable FP LWA (Case $\left.L_{A}=5.2 \lambda_{0}\right)$.

Finally, to give a better insight about the influence of the gain variation on the SPBW, Fig. 2.9.5(a) shows the measured gain radiation patterns at the three frequencies which define the FSPBW $f_{0}, f_{1}$ and $f_{2}$ for each case of Table 2.9.1. In the first state, the LWA operates close to broadside $\left(\theta_{R}=10^{\circ}\right)$, and the gain decreases rapidly when frequency is reduced due to the proximity to the lower cutoff regime of the leaky mode. This gain fluctuation can be verified in Fig. 2.9.5(b) (blue curve), which represents the relative variation of the measured gain at $\theta_{R}$ with frequency normalized w.r.t the maximum gain measured at $f_{0}$ for each case. The second state $\left(\theta_{R}=20^{\circ}\right)$ presents a softer decay of the gain when frequency is varied, thus obtaining higher bandwidth (green curve in Fig. 2.9.5(b)). The third state $\left(\theta_{R}=30^{\circ}\right)$ presents lower bandwidth as a consequence of the stronger sensitivity of $\theta_{R}$ with frequency (high scanning ratio) and especially the abrupt gain pattern variation as frequency is increased due to the proximity of this operating state to the upper stopband of the leaky mode (red curve of Fig. 2.9.5(b)), where also dissipative losses and mismatching take more effect. 

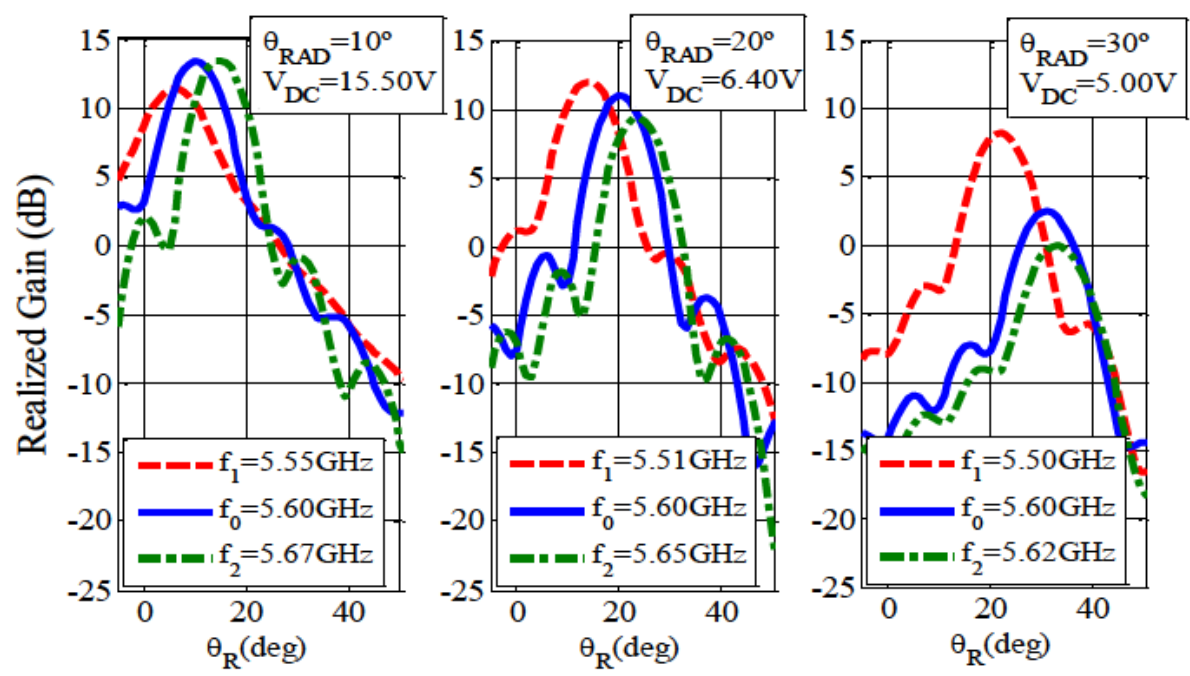

(a)

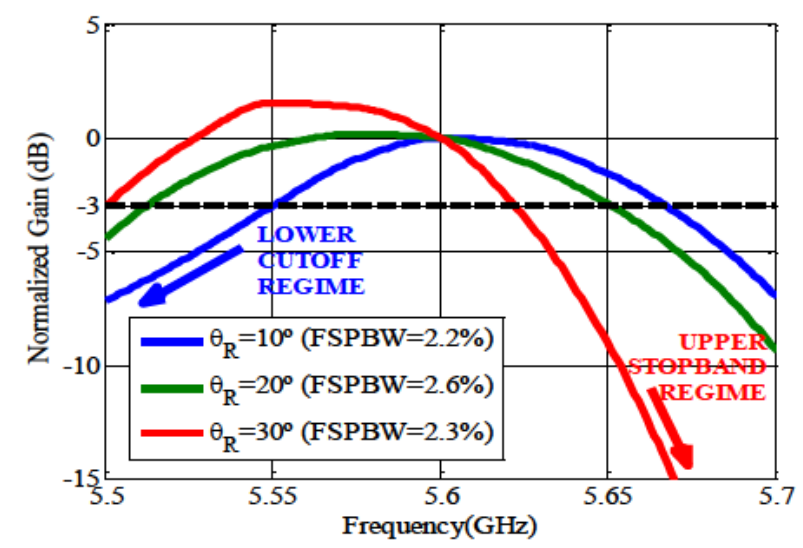

(b)

Fig. 2.9.5 a) Simulated Realized Gain patterns at $f_{1}, f_{0}$ and $f_{2}$ for different scanning configurations b) Variation of the realized gain (simulated from final prototype) for each case as a function of frequency (values normalized w.r.t. maximum gain measured at central design frequency $f_{0}=5.6 \mathrm{GHz}$ for each respective case). 


\subsection{Conclusions}

A new type of fixed-frequency electronically-steerable one-dimensional (1D) Fabry-Pérot (FP) LWA, which is based on a tunable Fabry-Perot (FP) cavity formed by a parallel-plate waveguide (PPW), a partially reflective surface (PRS), and a varactor-loaded tunable high impedance surface (HIS), has been presented in the chapter, fulfilling the first objective of this chapter (O1). The proposed reconfigurable LWA topology allows to electronically modify the resonant condition of the $\mathrm{TE}_{01}$ leaky mode inside the $\mathrm{FP}$ cavity, resulting in a variation of the pointing direction at a fixed frequency as the varactors' bias voltage is changed. Leaky-mode dispersion curves have been obtained from a simple but accurate Transverse Equivalent Network, which rigorously takes into account the length of the patches of the PRS and the effect of the varactors' junction capacitance in the phase response of the tunable HIS. The characterization of the PRS and HIS has been developed by the pole-zero methods proposed in [Maci 2005] and [García-Vigueras 2010]. Also, a re-adaptation of the modified pole-zero method presented in [García-Vigueras 2010], has been developed to characterize the tunable HIS. This characterization process will be also important for the analysis of the structures presented in next chapters. Thus, objectives $\mathbf{O 2}$ and $\mathbf{O 3}$ have been accomplished. These modal curves are very useful for the design of the main parameters of the antenna, in order to optimize the beam-scanning range. A prototype with $L_{A}=5.2 \lambda_{0}$ (at $5.6 \mathrm{GHz}$ ) has been fabricated and tested. Leaky-mode results are in very good agreement with full-wave simulations and experiments, which have shown a continuous scanning from $9^{\circ}$ to $30^{\circ}$ in a prototype operating at the fixed frequency of $5.6 \mathrm{GHz}$, as the varactors' bias voltage is varied from $18 \mathrm{~V}$ to $5 \mathrm{~V}$. The scanning range is limited in the endfire region due to the drop of the leakage rate and the increase of thermal losses and mismatching, which are associated with the perfect magnetic conductor (PMC) condition of the tunable HIS, as concluded from the efficiency analysis performed in Section 2.8. Also, it is observed that the antenna presents a radiation efficiency close to $70 \%$ for most part of the scanning range, which is reduced as the pointing angle scans to endfire. This reduction of efficiency is also noticed in the gain, which is reduced from $12 \mathrm{~dB}$ to $5 \mathrm{~dB}$ at $30^{\circ}$. The last objective of this chapter (O4) is achieved in Section 2.9 , where the pattern bandwidth of the antenna is analyzed. To this aim, a simple theoretical expression has been derived to approximate the pattern bandwidth of reconfigurable scanning LWAs, just from the information provided by the frequency dispersion curves of the structure. This formulation has been applied to the 1D FP LWA presented in this chapter, demonstrating that, despite the simplicity of the model, the predictions are quite in concordance with simulated results extracted from ideal (lossless) and perfectly matched case, and also are reasonably good when losses and mismatching are taken into account. From this study it was concluded that the main limiting factors which influence the maximum pattern bandwidth of a reconfigurable LWA are, 
apart from the natural dispersion of the guiding structure, the length of the antenna (and beamwidth) and the scanning range chosen to operate the antenna. A maximum scanning pattern bandwidth of $2.22 \%$ at $5.6 \mathrm{GHz}$ is estimated for the maximum scanning range associated to this antenna $\left(\left[9^{\circ}-30^{\circ}\right]\right)$.

The work developed in this chapter has been published in $\mathbf{3}$ peer-review international journal paper (IJ), 2 international conference papers (IC), 1 Spanish journal papers (SJ) and 1 Spanish conference paper (SC). These references are copied below; they have been extracted from the complete list of publications of Section 5.3.

IJ1. R. Guzman-Quirós, J. L. Gomez-Tornero, A. R. Weily, and Y. J. Guo, "Electronically steerable 1-D Fabry-Perot leaky-wave antenna employing a tunable high impedance surface," IEEE Trans. Antennas Propag., vol. 60, no. 11, pp. 5046 -5055, Nov. 2012.

IJ3. Y.J. Guo, J.L. Gomez-Tornero, R. Guzmán-Quirós, and A.R. Weily, "Reconfigurable Leaky-Wave Antennas," Forum for Electromagnetic Research Methods and Application Technologies (FERMAT), vol.1, no., pp., January 2014, available online : http://www.efermat.org/files/articles/1533721af95bd9.pdf

IJ4. R. Guzman-Quirós, J. L. Gomez-Tornero, A. R. Weily, and Y. J. Guo, "Dynamic Scanning Pattern Bandwidth in Electronically-Steerable Leaky-Wave Antennas," IEEE Antennas and Wireless Propagat. Lett., vol., no., pp., submitted.

IC3. R. Guzmán-Quirós, J. L. Gómez Tornero, M. García-Vigueras, A. R. Weily, Y. J. Guo, "Novel Topology of Fabry-Perot Electronically Steerable Leaky-Wave Antenna", 6th European Conference on Antennas and Propagation (EUCAP 2012), pp. 224-228, March 2012. (Invited contribution to a convened session about "Leaky-Wave Antennas" at EuCAP 2012)

IC5. R. Guzmán-Quirós, J. L. Gómez Tornero, M. García-Vigueras, A.R. Weily, Y. J. Guo, "Advances in electronically reconfigurable LWAs in Fabry-Pérot and SIW technologies", 7th European Conference on Antennas and Propagation (EUCAP 2013), pp. 224-228, April 2013. (Invited contribution to a convened session about "Emerging Antenna Technologies" at EuCAP 2013)

SJ1. R. Guzmán-Quirós, M. García Vigueras, A. J. Martínez Ros, J.L. Gómez Tornero, "Control electrónico del haz radiado en antenas leaky wave activas", IV Jornadas de Introducción a la Investigación (UPCT), Ed. UPCT, ISBN: 1888-8356, pp.94-96, May 2011.

SC3. R. Guzmán-Quirós, M. García-Vigueras, A. J. Martínez-Ros, J.L. Gómez-Tornero, "Reconfiguración Electrónica del Haz Radiado en Antenas Leaky Wave Basadas en HIS 
Activas", XXVI URSI (International Scientific Radiocommunications Union) Symposium, Madrid, Spain, Sept. 2011. 


\section{Chapter 3 Full-Space Electronic-Steering 1D FP LWA}

In this chapter we focus on the design of a full-space electronic scanning 1D FP LWA with steerability at both first (positive angles) and second (negative angles) quadrants. The half-space structure presented in Chapter 1 has been modified to achieve this new capability. In this case, the tunable HIS has been divided in two sections which varactors are independently biased and a central excitation is located at the middle of the waveguide. Dispersion analyses reveal two regions, the scanning region, which was previously employed to demonstrate the electronic scanning of the previous half-space 1D FP LWA, and an electromagnetic band-gap (EBG), which will be useful to switch the section of the antenna which we want to operate (or not) as a LWA. Thus, an EBG electronic-routing mechanism is conceived to reroute the waves excited from the central feeding to the left or right antenna section. Then, three operating modes are defined combining the operating regimes in which work each section: forward regime allows to scan at the first quadrant, backward regime allows to scan the beam at the second quadrant, and broadside radiation, to obtain a beam pointed at broadside $\left(\theta_{R A D}=0^{\circ}\right)$. A prototype has been fabricated to operate at $5.5 \mathrm{GHz}$ obtaining very good results between measurements, simulations and theory and demonstrating an electronically-controlled scanning region from $\theta_{R A D}=-25^{\circ}$ to $\theta_{R A D}=+25^{\circ}$, passing through broadside radiation. Next objectives are accomplished with this chapter: 
O5. Design of a reconfigurable 1D FP LWA with full-space electronic-steering employing a tunable HIS and the EBG electronic-routing mechanism.

O6. Demonstration of an electronic-routing mechanism based on the electromagnetic bandgap of periodic FP LWAs.

This chapter is organized as follows:

Section 3.1 presents an overview on full-space electronic-steering LWAs presented so far. Section 3.2 introduces the structure of proposed reconfigurable antenna. In Section 3.3, the dispersion curves shown in the last chapter for the 1D FP LWA are re-analyzed in order to define the scanning and the EBG dispersion regions as a function of the varactors' junction capacitance $\left(C_{j}\right)$. Then, the different operating regimes of the new full-space electronic-steering antenna are summarized in Section 3.4. Section 3.5 presents a fabricated prototype, operating at $5.5 \mathrm{GHz}$, and discusses measured results. The conclusions are summarized in Section 3.6. 


\subsection{Introduction}

In Chapter 1, electronic-steering capabilities were demonstrated for a 1D FP LWA employing a tunable HIS loaded inside. However, this antenna achieves scanning of its main beam just at positive angles (half-space electronic steering). Full-space electronic-steering is in general a more desirable feature for leaky-wave antennas (LWAs), since it allows to steer the beam from negative to positive angles, including broadside $\left(\theta_{R A D}\right)$ direction. Since the 1960 's, continuous frequency-scanning capability from backward to forward quadrant has been achieved by designing periodic LWAs ([Oliner 2007], [Jackson 2008]). However, research is now focused on fixed-frequency continuous full-space scanning devices since current wireless communication systems normally operate at a given operating frequency, as discussed previously. Recently, this capability has been achieved by inserting active components in different one-dimensional (1D) LWA technologies. For example, one of the most employed seems to be the composite right/left handed (CRLH) metamaterial line. These lines allow to excite from backward-to-forward leaky waves which allow to scan from negative (second quadrant) to positive (first quadrant) angles with frequency and also electronically at fixed frequency. Electronically scanning CRLH LWAs have been integrated in microstrip ([Lim 2004-I], [Lim 2004-II], [Matsuzawa 2005, 2006, 2007]), half-width microstrip ([Ouedraogo 2011]), or half-mode substrate-integrated waveguide (SIW) technology ([Suntives 2012]) to scan at fixed frequency. Also, electrically tunable surfaces have been employed to excite backward-to-forward waves modulating electronically the periodicity of the structure to radiate with the higher Floquet harmonic $n=-1$. In [Sievenpiper 2005], this is achieved employing tunable HIS loaded with varactors, whereas [Chicherin 2011-I, II] employ MEMS varactor arrays close to a silicon dielectric rod waveguide (DRW), as reconfigurable periodic LWAs. Similarly, [Manasson 2010] employs leaky waves diffracted by an electronically-controlled diffraction grating placed in the evanescent field of a dielectric waveguide, allowing beam steering. In [Esquius-Morote 2014], a tunable graphene LWA has been recently proposed. The graphene is electronically modulated along its aperture length, to achieve backward-to-forward scanning.

In general, achieving full-space scanning is more complicated than half-space steering, requiring more independently controlled diodes/switches to generate backward-to-forward leaky waves ([Lim 2004-II], [Sievenpiper 2005], [Ouedraogo 2011]), and very demanding design constraints to control the CRLH over the complete angular region ([Nguyen 2011]). In addition, broadside $\left(\theta_{R A D}=0^{\circ}\right)$ radiation is usually one of the issues to take into account for continuous full-space scanning optimization. Broadside can be achieved by several methods, but not by a simple natural uniform forward-scanning LWA excited from one end, since it would require to 
operate the waveguide at cutoff. To this aim, periodic antennas operating with the $n=-1$ harmonic have been employed ([Jackson 2008], [Oliner 2007]). However, these structures require of special designs since typically they start to present an open-electromagnetic band-gap (EBG) when they are close to point at boresight, ([Oliner 2007], [Jackson 2008]), which makes the travelling wave turn into a standing-wave antenna, reducing gain and increasing mismatching. The suppression of the open-EBG to optimize radiation at broadside is not trivial, although it has been overcome with some novel designs in printed technology to achieve continuous backward-to-forward frequency scanning ([Paulotto 2009]). In the case of CRLH metamaterial LWAs, which operate with the fundamental mode of the line, broadside can be also achieved; however the line must be properly designed to accomplish the balanced condition ([Caloz 2005]). Also, a half-space 1D LWA operating with the simple fundamental mode and excited from the center can be employed to generate a broadside beam ([Burghignoli 2006], [García-Vigueras 2012-II], [Jackson 2008], [Lovat 2006-I]). In this configuration, the central source excites two leaky waves propagating in opposite directions which beams can merge in one single directed beam pointed at broadside when the structure is operated close to (but slightly below) cutoff. Thus, broadside is achieved as long as it is satisfied the splitting condition $\beta=\alpha$ ([Lovat 2006-I]). If this condition is not satisfied, then two separated beams arise pointing at opposite directions, reducing the gain at broadside, what makes this configuration unpractical for scanning applications. In order to avoid complex designs, another simple configuration consists of a single half-space LWA excited independently from both ends ([Nguyen 2011], [Wang 1999, 2000]). This is the case presented in [Nguyen 2011], [Wang 2000], which proposes a simpler end-switched topology which employs a two-port half-space electronic-steering CRLH LWA and a single-pole double-throw (SPDT) switch to choose the radiation quadrant. In these configurations, only a half-space electronic scanning antenna is needed, but excited from both ports at the far ends of the structure. Thus, the design constraints are strongly simplified.

An even simpler approach is proposed in this chapter, so full-scanning capability is achieved without SPDT switch. In this case, a central-fed (non-metamaterial) LWA, like the presented in Chapter 1 , is proposed. To avoid the dual beam generated when $\beta>\alpha$, a novel electronic-routing mechanism is employed based on the electromagnetic band-gap (EBG) properties of the FP cavity, so the centrally-excited LWA is able to drive forward leaky waves into right or left direction, allowing scanning into $1^{\text {st }}$ or $2^{\text {nd }}$ quadrant respectively, besides radiation at broadside by satisfying the aforementioned splitting condition. 


\subsection{Structure}

The scheme of the proposed configuration is illustrated in Fig. 3.2.1. In the previous section, it was shown how a 1D FP LWA can be loaded with a tunable high impedance surface (HIS) to obtain half-space electronic beam steering from $+9^{\circ}$ to $+30^{\circ}$.

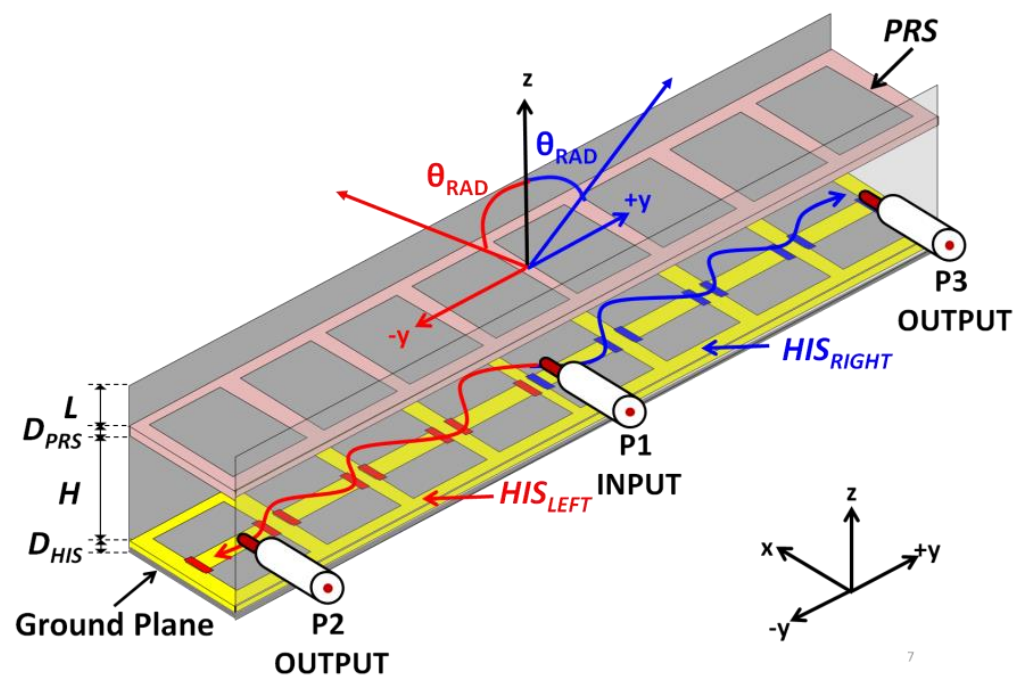

Fig. 3.2.1 Scheme of the proposed full-space steering 1D FP LWA. Same dimensions of original 1D FP LWA are considered ( $a=25 \mathrm{~mm}, H=25.2 \mathrm{~mm}, L=13.6 \mathrm{~mm}, D_{H I S}=1.524 \mathrm{~mm}$, $\varepsilon_{r H I S}=3, P_{H I S}=30 \mathrm{~mm}, Q_{H I S}=14 \mathrm{~mm}, L_{H I S}=17 \mathrm{~mm}, g=1 \mathrm{~mm}, D_{P R S}=0.8 \mathrm{~mm}, \varepsilon_{r P R S}=4.4, P_{P R S}=20 \mathrm{~mm}$,

$$
\left.Q_{P R S}=18 \mathrm{~mm}, L_{P R S}=22 \mathrm{~mm}\right) \text {. }
$$

The input port of the previous electronically-steerable 1D FP LWA was located at one end of the 1D FP leaky waveguide. This original structure is modified here by locating the input port at the middle of the waveguide (P1 in Fig. 3.2.1), and integrating two independently tuned HIS located at each side of the center feeding (HIS $S_{L E F T}$ and $H I S_{R I G H T}$ in Fig. 3.2.1). Therefore, the structure is split in two independently controlled 1D FP LWAs respectively excited by two leaky waves which propagate in opposite directions (+y and $-y$ in Fig. 3.2.1) from the central feed. As will see in next section, the independent control of the dispersion at each side of the antenna will allow scanning at forward (positive quadrant), backward (negative quadrant) or broadside directions. 


\subsection{Dispersion Analysis and Defined Regions}

Below, the dispersion curves of the leaky-mode complex propagation constant as a function of the HIS varactors' junction capacitance $\left(C_{j}\right)$ are presented in Fig. 3.3.1 for a fixed frequency of $5.5 \mathrm{GHz}$.

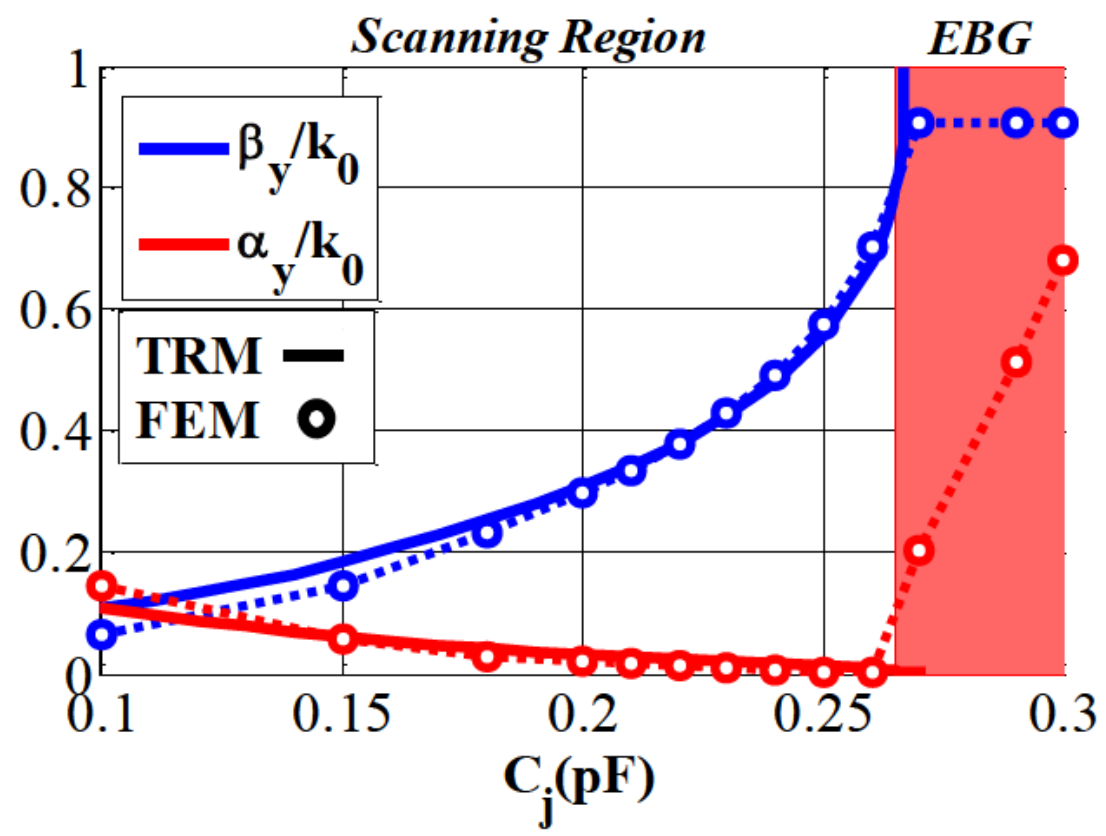

Fig. 3.3.1 Defined regions for the leaky-mode dispersion curves obtained for the half-space electronic-steering 1D FP LWA as a function of $C_{j}$ at $5.5 \mathrm{GHz}$.

Such results have been obtained from the TEN used for the original half-space steering 1D FP LWA analyzed in the previous section, and have been validated from numerical full-wave simulations ([CST 2010]) with the finite structure of $L_{A}=5.2 \lambda_{0}$ at $5.5 \mathrm{GHz}$ excited from one end. In this case, Fig. 3.3.1 shows the dispersion curves for a $C_{j}$ range which extends from $C_{j}=0.1 \mathrm{pF}$ to $C_{J}=0.3 p F$, so two well-differentiated regions can be outlined:

A. Scanning region: The region is defined in the range $C_{j}=[0.1 \mathrm{pF}, 0.27 \mathrm{pF}]$. Here, the TE leaky mode propagates in the structure $(\beta<\alpha)$ and the radiation condition is satisfied $\left(\beta_{y} / k_{0}<1\right)$, so the antenna is radiating at a certain pointing angle $\theta_{R A D}$ ([Oliner 2007]). In this region, $\beta_{y}$ (and so $\left.\theta_{R A D}\right)$ is tuned as a function of the varactors' bias voltage $\left(C_{j}\left(V_{R}\right)\right)$. In particular, at $5.5 \mathrm{GHz}$ the LWA provides half-space steering from $\theta_{R A D} \approx 6^{\circ}\left(\beta_{y} / k_{0} \approx 0.1\right)$ for $C_{j}=0.1 \mathrm{pF}$ to $\theta_{R A D} \approx 60^{\circ}\left(\beta_{y} / k_{0} \approx 0.9\right)$ for $C_{j}=0.27 \mathrm{pF}$.

B. Electromagnetic band-gap (EBG) region: An electromagnetic band-gap (EBG) region for $C_{J}=[0.27 \mathrm{pF}, 0.3 \mathrm{pF}]$ (red shaded area in Fig. 3.3.1) is identified. The propagation of the leaky mode along the FP cavity is prevented inside this stop-band, as shown by the strong rise of 
the attenuation constant $\alpha_{y} / k_{0}$ and the null value of $d \beta_{y} / d C_{j}$. Nevertheless, this increment of $\alpha_{y}$ does not correspond to an increment in the leakage rate, but to the reactive attenuation constant, since no power is carried by any propagating mode inside this region ([Oliner 2007], [Jackson 2008]).

From this dispersion analysis, it is concluded that a FP cavity can operate either into the Radiating or $E B G$ region, depending on the operating point $\left(C_{j}\right)$ in which the reconfigurable antenna is tuned. If the antenna operates into the scanning region, a leaky wave will be propagated by the FP cavity, radiating its power at a radiation angle determined by $C_{j}$. On the contrary, if the antenna is operated inside the EBG region, propagative modes will be prevented and its energy will be mostly reflected back to the source, as will see in next section.

Other EBG structures have been also proposed to modify the aperture of antennas ([Boutayeb 2006-I, II, III, IV, 2007-I, II], [Ettorre 2007], [Llombart 2005, 2007], [Neto 2006]). Boutayeb ([Boutayeb 2006-I, III, IV, 2007-I, II]) employed low cost cylindrical EBG (CEBG) structures made of circular arrays of metallic wires. Thus, directional antennas with potential reconfigurable capability are envisaged, excited by a punctual omnidirectional source and controlling the CEBG by defects on the wires. Thus, electronically reconfigurable antennas with beamwidth electronic control or azimuthal beam-scanning have been achieved applying the same principles with tunable CEBG loaded with PIN diodes ([Boutayeb 2004, 2006-II], [Djerafi 2008], [Edalati 2009], [Edalati 2011]). In [Llombart 2005, 2007] and [Neto 2006], planar circularly symmetric EBG (PCS-EBG) structures were employed to suppress surface waves and conceive printed antennas with high bandwidth and enhanced directivity. Similar PCS-EBG structures have been employed in [Ettorre 2007] to realize directional antennas on printed circuit board technology, by controlling the dispersion of the surface waves launched by a punctual source. 


\subsection{Operating Regimes}

In this section are explained the different regimes in which the antenna can be operated. A lossless prototype with an aperture length of $L_{A}=5.2 \lambda_{0}$ excited by a coaxial in the middle of the waveguide has been simulated by FEM ([CST 2010]). Analysis of the electric field distribution inside the FP cavity for each operating mode and the associated radiation patterns are shown in Fig. 3.4.1 and Fig. 3.4.2 respectively.

As previously explained, it is possible to control independently each side of the centrallyexcited 1D FP LWA biasing each defined tunable HIS $\left(H I S_{L}, H I S_{R}\right)$, allowing to operate each FP cavity in scanning or EBG region separately. Combining the operating region in which works each FP cavity, three operation regimes have been defined:

A. Backward scanning regime. To scan at negative angles, HIS $\mathrm{S}_{\mathrm{LEFT}}$ must be tuned inside the scanning region $\left(C_{j L}<0.27 \mathrm{pF}\right)$ while $\mathrm{HIS}$ RIGHT is fixed at EBG region $\left(C_{j R}=0.3 \mathrm{pF}\right)$. In this way the input signal is guided to the left side of the LWA and no energy travels to the right side, as it can be seen in Fig. 3.4.1(a). By properly tuning $C_{j L}$ in the range [0pF, $0.23 \mathrm{pF}$ ], a single beam pointing at negative angles should be theoretically scanned in the angular region $\theta_{R A D}=\left[-5^{\circ},-25^{\circ}\right]$. Three radiation patterns corresponding to three different configurations of the antenna in this operating mode are shown in Fig. 3.4.2(a).

B. Forward scanning regime. This is the symmetrical case, as observed in Fig. 3.4.1(b). HIS $_{\text {LEFT }}$ is now operated in the EBG regime $\left(C_{j L}=0.3 \mathrm{pF}\right)$ and HIS $\mathrm{SIGHT}_{\text {in }}$ is tuned in the scanning region to provide steering at positive angles. In this case, with $C_{j R}=[0 \mathrm{pF}, 0.23 \mathrm{pF}]$ the beam is theoretically steered in the range $\theta_{R A D}=\left[+5^{\circ},+25^{\circ}\right]$. Symmetric response is verified observing the electric fields in Fig. 3.4.1(b) and the radiation patterns obtained for three symmetrical configurations operating in the forward regime shown in Fig. 3.4.2(b).

C. Broadside radiation. Optimum radiation at boresight can be obtained by launching two opposite-directed leaky waves which satisfy the splitting condition $\beta_{y} / k_{0}=\alpha_{y} / k_{0}$ ([Lovat 2006-I]). At this point, maximum directivity at broadside is achieved, when the two beams generated by both left and right FP LWAs, each one pointing slightly above broadside, merge each other to form one broadside beam. To this end, HIS LEFT $_{\text {and }}$ HIS $_{\text {RIGHT }}$ are tuned at the same operating point inside the scanning region with $C_{j R}=C_{j L}=0.1 \mathrm{pF}$, which satisfies this condition according to Fig. 3.3.1. Above this condition, two symmetric beams will arise scanning regularly as a function of $C_{j}$. Fig. 3.4.1(c) illustrates the electric field distribution generated for this operating regime when $C_{j L}=C_{j R}=0.1 \mathrm{pF}$, showing how the fields on the two 
sides of the antenna converge to form a single beam radiating at $\theta_{R A D}=0^{\circ}$, as observed in Fig. 3.4.2(c). Good explanation, discussion and applications of this condition can be found in ([Ip 1990], [Yamamoto 1999], [Lovat 2006-I], [Sutinjo 2008] and [Jackson 2008]).

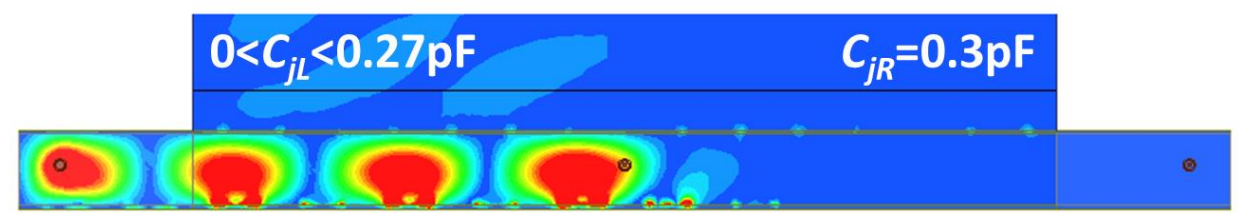

(a)

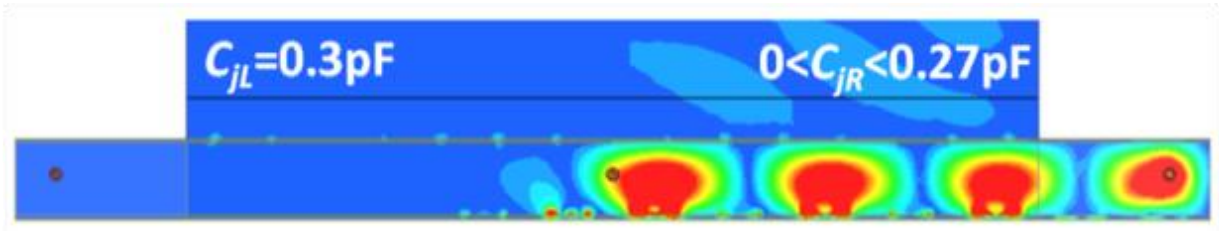

(b)

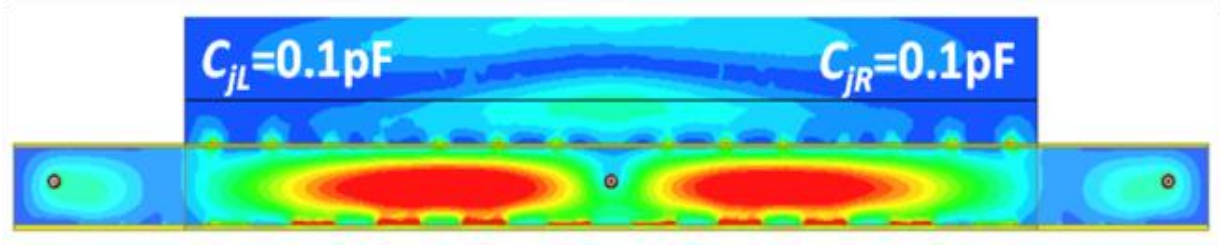

(c)

Fig. 3.4.1 Electric field distribution along the centrally excited reconfigurable 1D FP LWA obtained by FEM ([CST 2010]) for the three operating regimes: (a) Backward scanning (b) Forward scanning, (c) Broadside radiation.

As observed, the EBG regime can be employed as an electronic-routing mechanism which allows to reroute the energy introduced from the central coaxial to the desired side of the antenna. It is noteworthy to advance that this EBG electronic-routing concept will be of interest, not to obtain full-space 1D FP LWAs which scans its fan beam in elevation, but to conceive a more interesting type of antenna consisted of a centrally-excited two-dimensional (2D) FP LWAs with two-dimensional electronic beam-scanning (in elevation and azimuth) of its pencil beam, as will see in next section. In the next subsections, before presenting the 2D structure, experiments with a prototype of the full-space 1D FP LWA demonstrate this concept and also the advantages and disadvantages of this type of reconfiguration mechanism. 


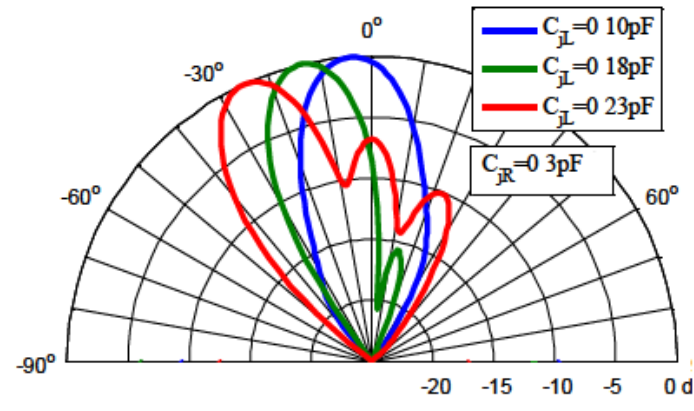

(a)

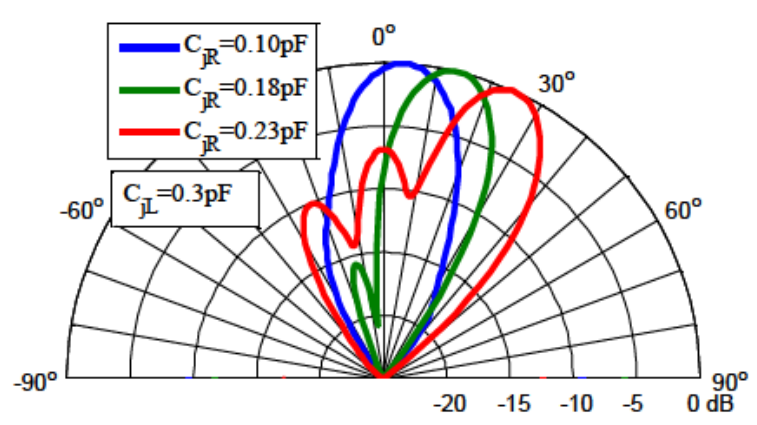

(b)

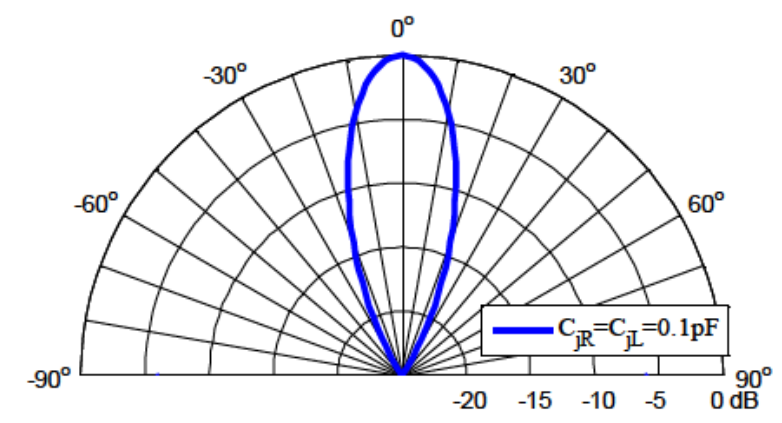

(c)

Fig. 3.4.2 Normalized H-plane radiation patterns obtained by FEM ([CST 2010]) at 5.5GHz for different $\left(C_{j L}, C_{j R}\right)$ operating configurations: (a) Backward scanning (b) Forward scanning, (c) Broadside radiation. 


\subsection{Experiments}

\subsubsection{Fabricated Prototype}

The optimized 3D model simulated to check the final response of the full-space scanning 1D FP LWA and a photograph of the fabricated prototype are shown in Fig. 3.5.1. The prototype has been fabricated from the prototype of the half-space steering 1D FP LWA presented in the previous section. So, same varactors, bias network and dimensions of the previous structure can be considered. However, some structures modifications have been introduced to obtain the new full-space structure. A coaxial input port has been located at the center (P1) of the waveguide, whereas the two ports located at the far ends have been left as the output ports at each side of the antenna respectively (P2 and P3 in Fig. 3.5.1(b)). The tunable HIS bias network employed for the 1D FP LWA has been isolated at the center of the waveguide, so two independently controlled tunable HIS are now defined. Fig. 3.5.1(b) also shows the cables that supply the control signal voltages to each side of the HIS, defined as HIS LEFT $_{\text {and }}$ HIS $_{\text {RIGHT }}$, and associated to the DC bias voltages $V_{L}$ and $V_{R}$ respectively.

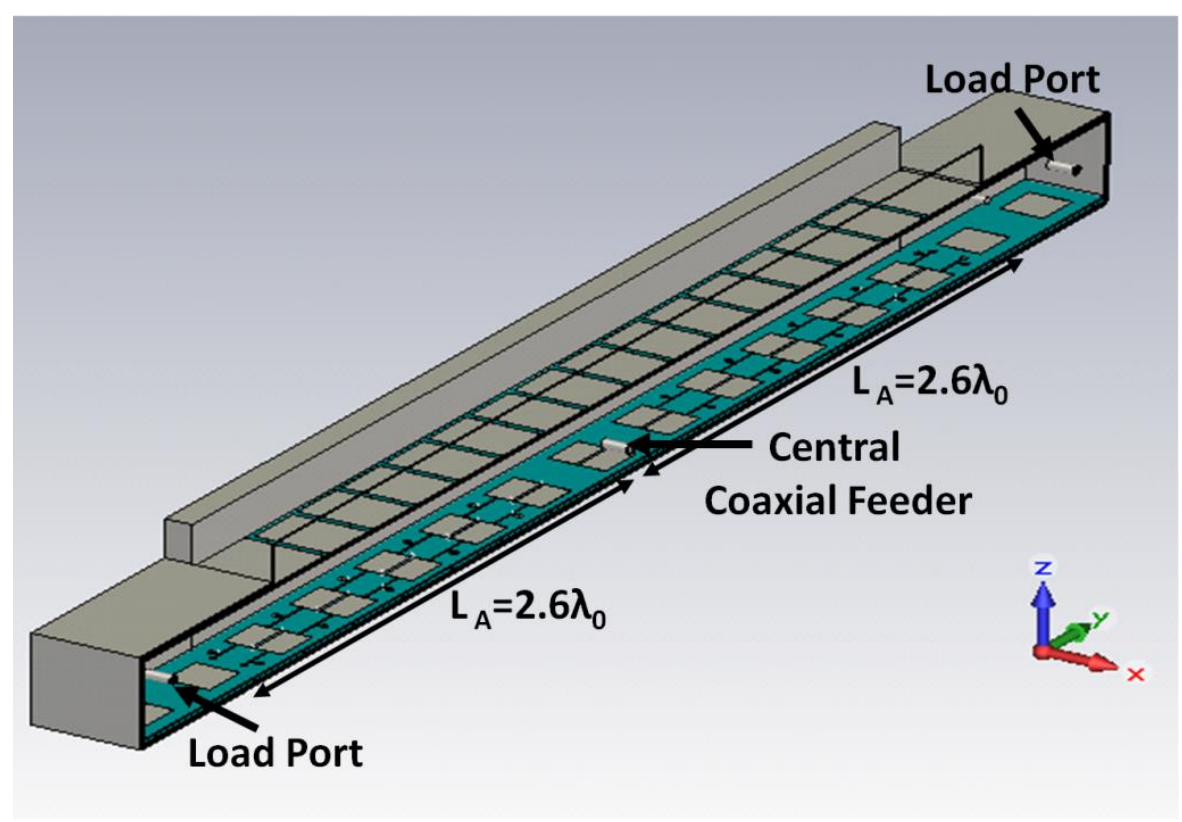

(a) 


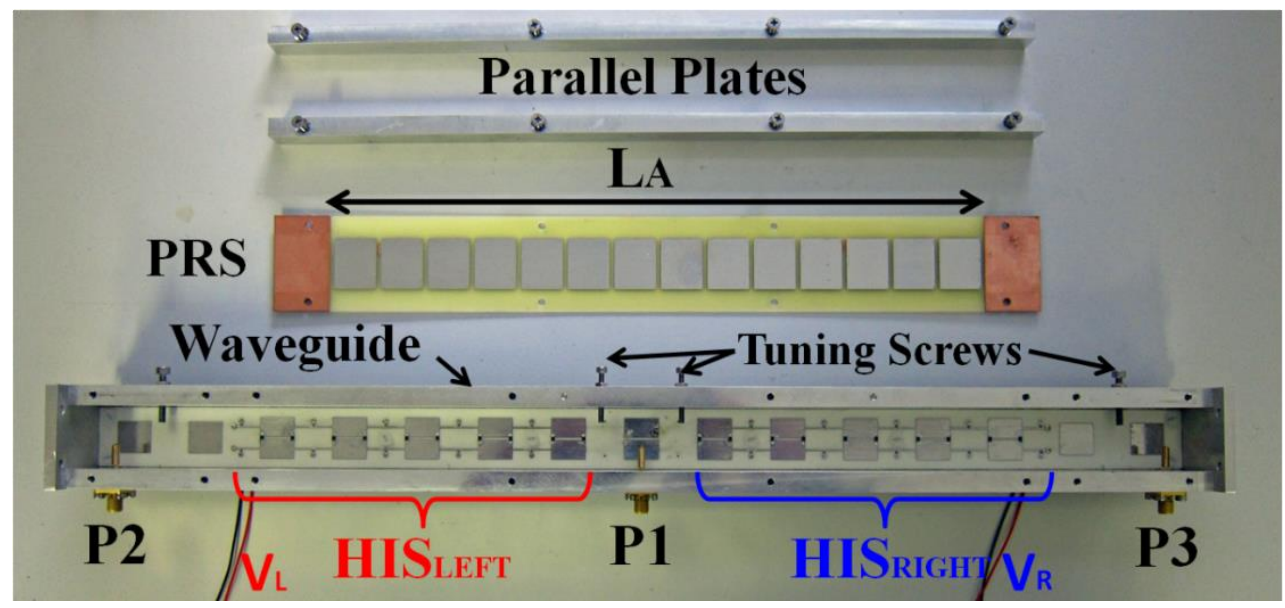

(b)

Fig. 3.5.1 (a) 3D Model of the final design of reconfigurable full-space electronic-steering 1D FP LWA excited from the center of the waveguide ([CST 2010]) and (b) Manufactured fullspace scanning 1D FP LWA prototype $\left(L_{A}=5 \lambda_{0}\right)$.

Regarding the central coaxial feeder used to excite symmetrically the structure, again a fullwave optimization process has been employed to improve the input matching of the antenna. Fig. 2.7.3 shows the schematic modeled in the full-wave simulator ([CST 2010]) of the coaxialfeeder introduced at the center of the FP waveguide. The vertical position of the central coaxial probe has been firstly optimized to obtain good input matching for the whole range of operating configurations in which the antenna can work. Later, two tuning screws have been symmetrically placed a both sides of the coaxial at a distance A (see Fig. 2.7.3(b)), which position, width (E2) and length (C2) has been optimized to enhance the response for the forward and backward scanning regimes. Finally, a hat has been added at the end of the inner conductor of the coaxial, inside the FP waveguide, in order to improve more the overall response.

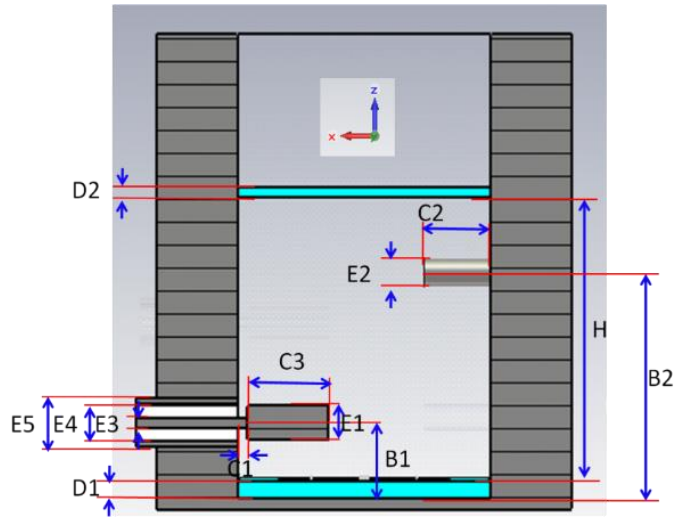

(a)

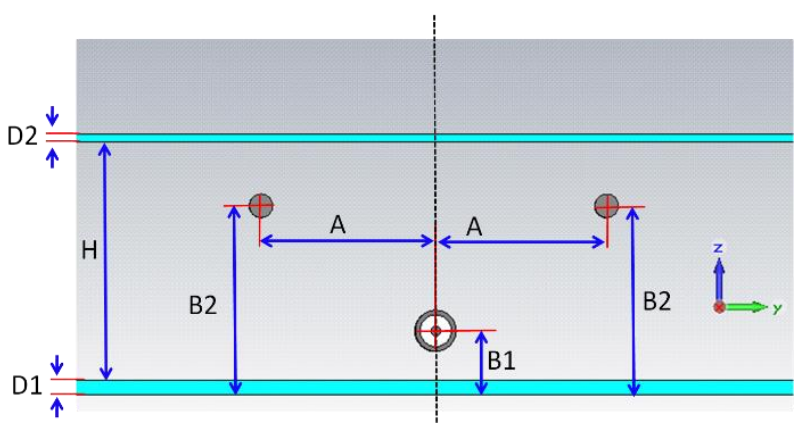

(b) 


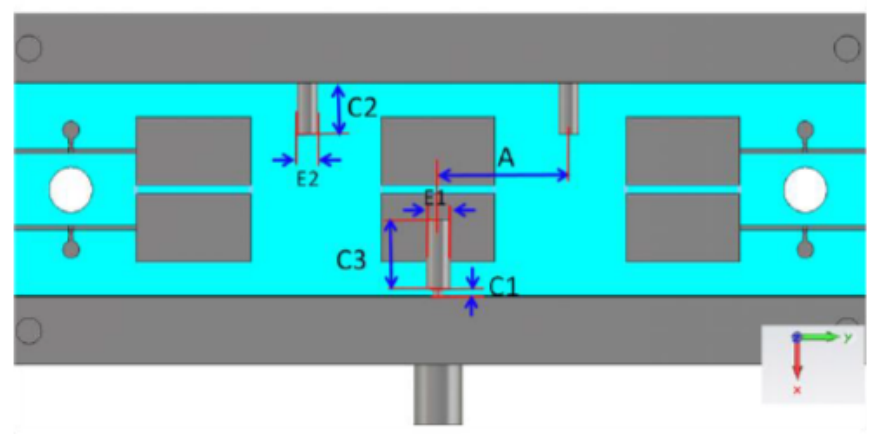

(c)

Fig. 3.5.2 Central coaxial feeder model ([CST 2010]). (a) Side view, (b) Front view and (c) Top view.

\begin{tabular}{|c|c|c|c|}
\hline PARAM. & DIM (mm) & PARAM. & DIM (mm) \\
\hline A & 16 & D2 & 0.8 \\
\hline B1 & 7.5 & E1 & 3 \\
\hline B2 & 13 & E2 & 2.5 \\
\hline C1 & 1 & E3 & 0.64 \\
\hline C2 & 6 & E4 & $2.05\left(\varepsilon_{\mathrm{r}}=2.1\right)$ \\
\hline C3 & 8 & E5 & 3 \\
\hline D1 & $1.524\left(\varepsilon_{\mathrm{r}}=3\right)$ & H & 25.2 \\
\hline
\end{tabular}

Table 3.5.1 Dimensions of the optimized coaxial-waveguide feeding network. 


\subsubsection{Experimental Results}

The theoretical and measured scanning response at $5.5 \mathrm{GHz}$ is plotted in Fig. 3.5.3, showing the variation of the pointing angle $\theta_{R A D}$ as a function of the varactor junction capacitance $C_{j}$ (for theoretical results obtained from the TRM and full-wave simulations), and also as a function of the bias voltage $V$ (for experiments). As explained in Section 4.2, the correspondence between $C_{j}$ and $V$ in this double-x-axis is obtained with a calibration of the varactors. It is important to notice that this double $\mathrm{x}$-axis in Fig. 3.5.3 is divided into three regions which correspond to the three aforementioned operation regimes of the electronically controlled FP LWA. The left side of Fig. 3.5.3 represents the backward scanning configuration, for which HIS $\mathrm{RIGHT}_{\text {is }}$ is biased with fixed $V_{R}=3.7 \mathrm{~V}$ (corresponding to $C_{j R}=0.3 \mathrm{pF}$ for EBG operation), while HIS $\mathrm{LEFT}_{\text {in }}$ is controlled with $V_{L}$ varying in the range $V_{L}=[4.1 \mathrm{~V}, 10.6 \mathrm{~V}]$ (which corresponds to $C_{j L}=[0.27 \mathrm{pF}, 0.1 \mathrm{pF}]$ to operate in the scanning region). The symmetrical biasing configuration is represented in the right side of Fig. 3.5.3, showing a fixed value of $V_{L}=3.7 \mathrm{~V}$ and $C_{j L}=0.3 \mathrm{pF}$ (HIS $\mathrm{LEFT}_{\mathrm{LET}}$ at EBG) and varying $V_{R}=[4.1 \mathrm{~V}, 10.6 \mathrm{~V}]$ and $C_{j R}=[0.27 \mathrm{pF}, 0.1 \mathrm{pF}]$ to obtain forward scanning.

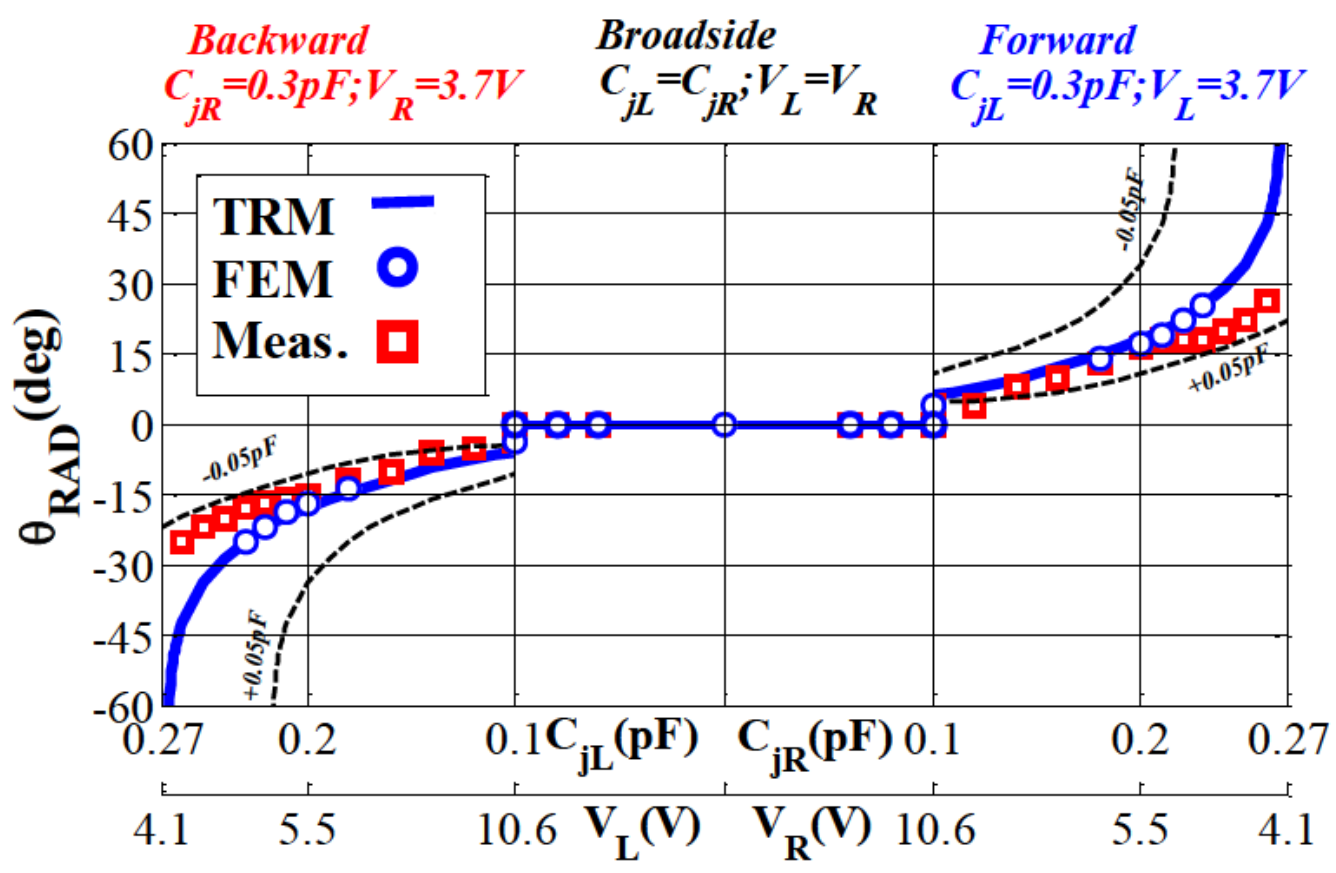

Fig. 3.5.3 Measured and simulated (FEM) scanning response for the full-space electronicsteering 1D FP LWA prototype at $5.5 \mathrm{GHz}$.

Finally, the central section in Fig. 3.5.3 represents the broadside configuration, for which symmetrical biasing is applied with $V_{L}=V_{R}=[10.6 \mathrm{~V}, 18 \mathrm{~V}]$ and $C_{j L}=C_{j R}=[0.1 \mathrm{pF}, 0.06 \mathrm{pF}]$. Very good agreement is observed for the theoretical scanning response obtained with TRM and fullwave commercial software ([CST 2010]), obtaining a scanning range of $\theta_{R A D}=\left[-60^{\circ},+60^{\circ}\right]$. Experimental results are plotted with red rectangles, showing good matching with theory for the 
angular region $\left[-15^{\circ},+15^{\circ}\right]$, but limiting the measured scanning range to $\left[-25^{\circ},+25^{\circ}\right]$. These discrepancies can be attributed to the varactors tolerances, already discussed in previous section. Actually, the measured scanning response lies inside the theoretical scanning response obtained when applying a deviation of $\pm 0.05 \mathrm{pF}$ to the junction capacitance $C_{j}$, as shown with dashed lines in Fig. 3.5.3. Nevertheless, Fig. 3.5.3 demonstrates that a backward-to-forward electronic scanning (including broadside radiation) can be obtained by using the proposed full-scanning configuration for FP LWAs.

The measured gain patterns of the fabricated antenna operating at backward, forward, and broadside configurations are plotted in Fig. 3.5.4. Fig. 3.5.4(a) illustrates the electronic scanning at negative angles, showing a single main beam scanned from $\theta_{R A D}=-25^{\circ}$ to $\theta_{R A D}=-5^{\circ}$ as $\mathrm{HIS}_{\mathrm{LEFT}}$ is tuned from $V_{L}=4.2 \mathrm{~V}\left(C_{j}=0.26 \mathrm{pF}\right)$ to $V_{L}=10.6 \mathrm{~V}\left(C_{j}=0.1 \mathrm{pF}\right)$, while HIS RIGHT is fixed at $V_{R}=3.7 \mathrm{~V}$ $\left(C_{J}=0.3 \mathrm{pF}\right)$. In Fig. 3.5.4(b), the scanning response at positive angles is shown, observing a symmetrical steering from $\theta_{R A D}=+5^{\circ}$ to $\theta_{R A D}=+25^{\circ}$ by controlling HIS and $V_{R}=4.2 \mathrm{~V}$ and keeping $\mathrm{HIS}_{\mathrm{LEFT}}$ at $V_{L}=3.7 \mathrm{~V}$. Both scanning regimes present a maximum gain of $G_{\max }=11.6 \mathrm{~dB}$ for $\theta_{R A D}= \pm 5^{\circ}$.

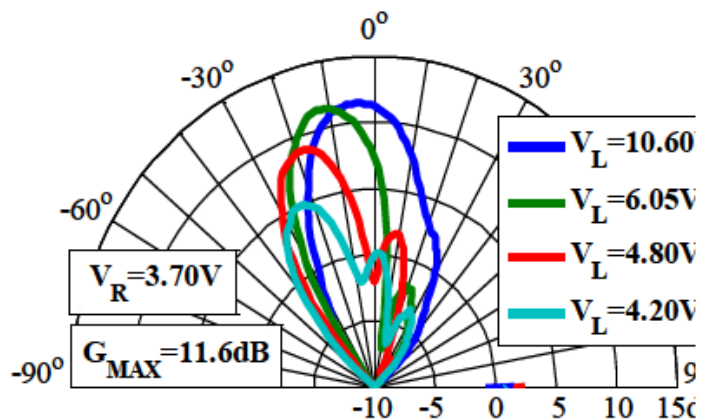

(a)

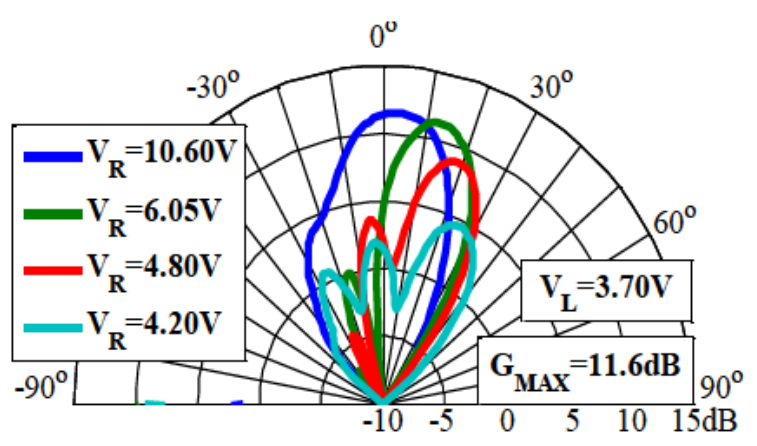

(b)

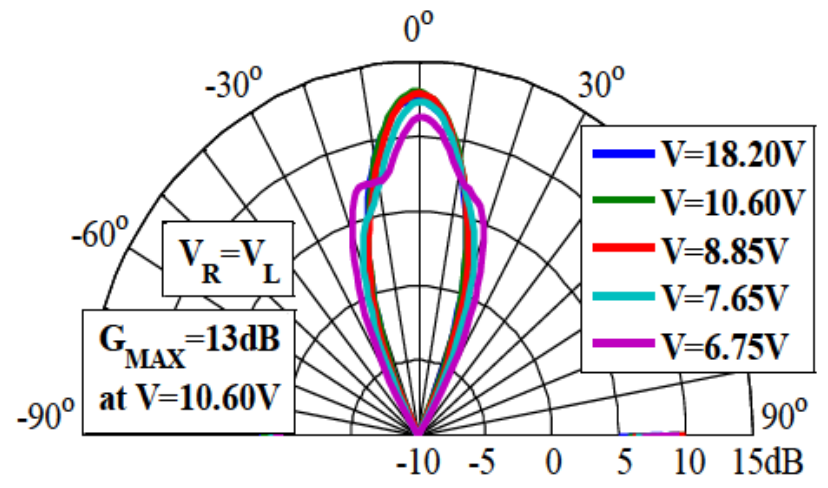

(c)

Fig. 3.5.4 Measured gain patterns for the full-space scanned 1D FP LWA at 5.5GHz for the three operating configurations.

However, slight asymmetries can be observed between the scanning responses for the negative and positive quadrants: this is most likely due to manufacturing tolerances and variation in the 
performance of the varactors for each HIS circuit. In particular, higher side lobes can be seen when scanning toward forward endfire than towards backward endfire, resulting in gain values of $G=5 \mathrm{~dB}$ for $\theta_{R A D}=-25^{\circ}$ and $G=4.5 \mathrm{~dB}$ for $\theta_{R A D}=+25^{\circ}$. This gain fall as the pointing angle is scanned caused by several loss factors such as the leakage rate drop, dielectric and varactor dissipation and mismatch, as commented in the efficiency analysis presented in Section 2.8. Nevertheless, the concept of using the EBG regime of the FP leaky line to guide the input energy to the desired quadrant is experimentally demonstrated, resulting in a main beam scanned in the corresponding radiation quadrant while preventing radiation in the opposite direction.

Fig. 3.5.4(c) is dedicated to the broadside radiation operation. As described before, the same bias voltage must be applied to both HIS circuits, $V_{L}=V_{R}$. Particularly, the splitting condition is satisfied for $V=10.6 \mathrm{~V}$ (which theoretically corresponds to $C_{j}=0.1 \mathrm{pF}$ where $\beta_{y} / k_{0}=\alpha_{y} / k_{0}$ in Fig. 3.3.1, obtaining a single beam radiating at broadside with maximum gain $G_{m a x}=13 \mathrm{~dB}$. Moreover, it is shown that the voltage can be varied in the range $V=[18.2 \mathrm{~V}, 6.75 \mathrm{~V}]$, and still a single beam at boresight is obtained. However, the gain decreases and side lobes start to appear as we move further from the splitting condition ([Lovat 2006-I]), as it can be seen in Fig. 3.5.4(c) for $V=6.75 \mathrm{~V}$ (that corresponds to $C_{j}=0.16 \mathrm{pF}$, where $\beta_{y} / k_{0}=0.2>\alpha_{y} / k_{0}=0.05$ in Fig. 3.3.1), which provides lower gain at broadside $G=11.3 \mathrm{~dB}$. Thus, it is experimentally checked that optimum radiation at broadside is obtained when both HIS are tuned at the splitting condition. The radiation patterns in Fig. 3.5.4 experimentally demonstrate backward-to-forward electronic scanning of the proposed FP antenna.

At this point, it should be stressed that when the antenna is operating at backward or forward operating modes, half of the aperture length remains unused. This is clearly observed from the field distributions depicted in Fig. 3.4.1(a) and Fig. 3.4.1(b). Therefore, the initial aperture length of $L_{A}=5.2 \lambda_{0}$ is reduced to $L_{A}=2.6 \lambda_{0}$, what produces a wider main beam and so decrement of the total directivity/gain of the antenna. On the contrary, this effect is not happening when the broadside configuration is tuned $\left(C_{j L}=C_{j R}\right)$. This operating mode is using both sides of the antenna and, if the PRS has been properly designed to illuminate the total aperture length with a high aperture efficiency (which depends on the leakage constant $\alpha_{y}$ of the excited leaky mode, as previously commented ([García-Vigueras 2012-II])), a narrower beamwidth, and so higher directivity (and gain), will be obtained at broadside direction than at the other configurations, as observed in Fig. 3.5.4.

Fig. 3.5.5(a) shows the input matching of the full-space scanning FP LWA as a function of the biasing voltage $V$ (and corresponding $C_{j}$ ) at the fixed design frequency of $5.5 \mathrm{GHz}$. As aforementioned, the matching circuit makes use of tuning screws (which can be seen in Fig. 
3.5.1), which were optimized to provide low $S_{11}$ for most of the scanning range including broadside. The antenna presents $S_{11} \approx-8 \mathrm{~dB}$ in the broadside radiation configuration, while in the backward and forward scanning regimes the mismatch increases from $-15 \mathrm{~dB}$ to $-5 \mathrm{~dB}$ as the beam is steered towards endfire. Similar tendency is observed with CST simulations and measurements. Some asymmetry between backward/forward scanning is again observed in measured $S_{11}$. Again, it is worth to mention that matching of reconfigurable antennas is a difficult issue to deal with. The full-space scanning FP LWA has been more difficult to match than its half-space counterpart because it operates in three different scanning regimes, with dramatic changes in the leaky-mode field distribution. As a result, matching is worse than in the half-space design.

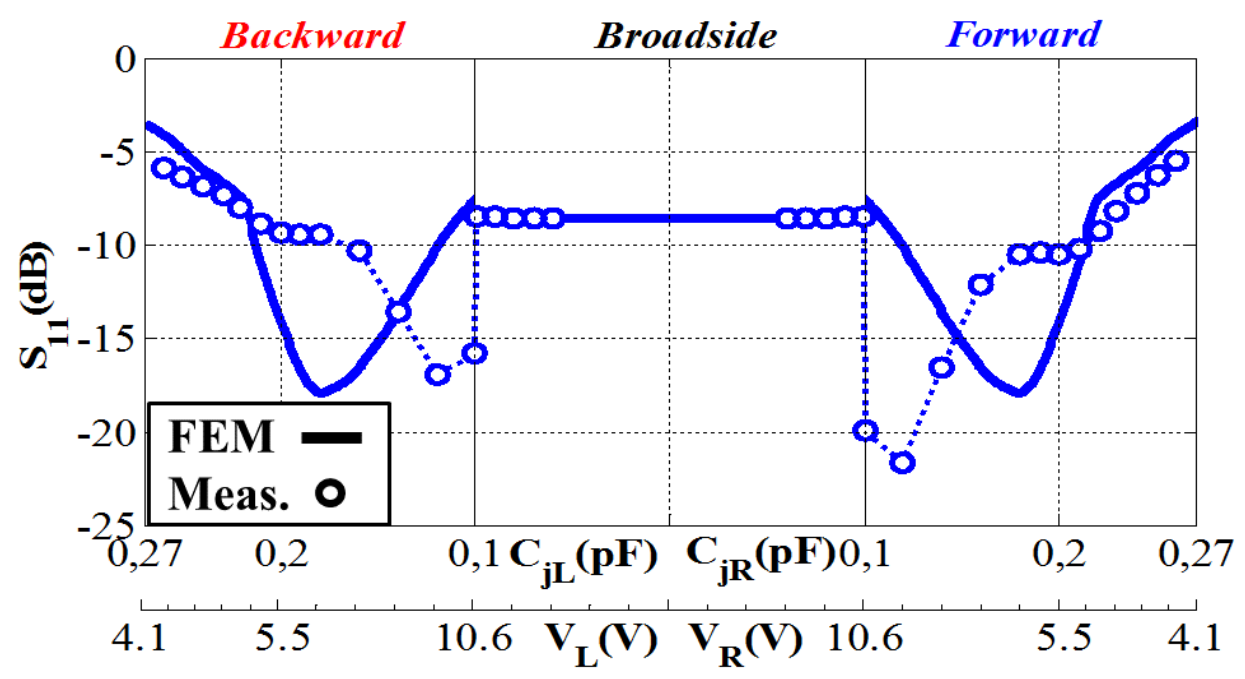

(a)

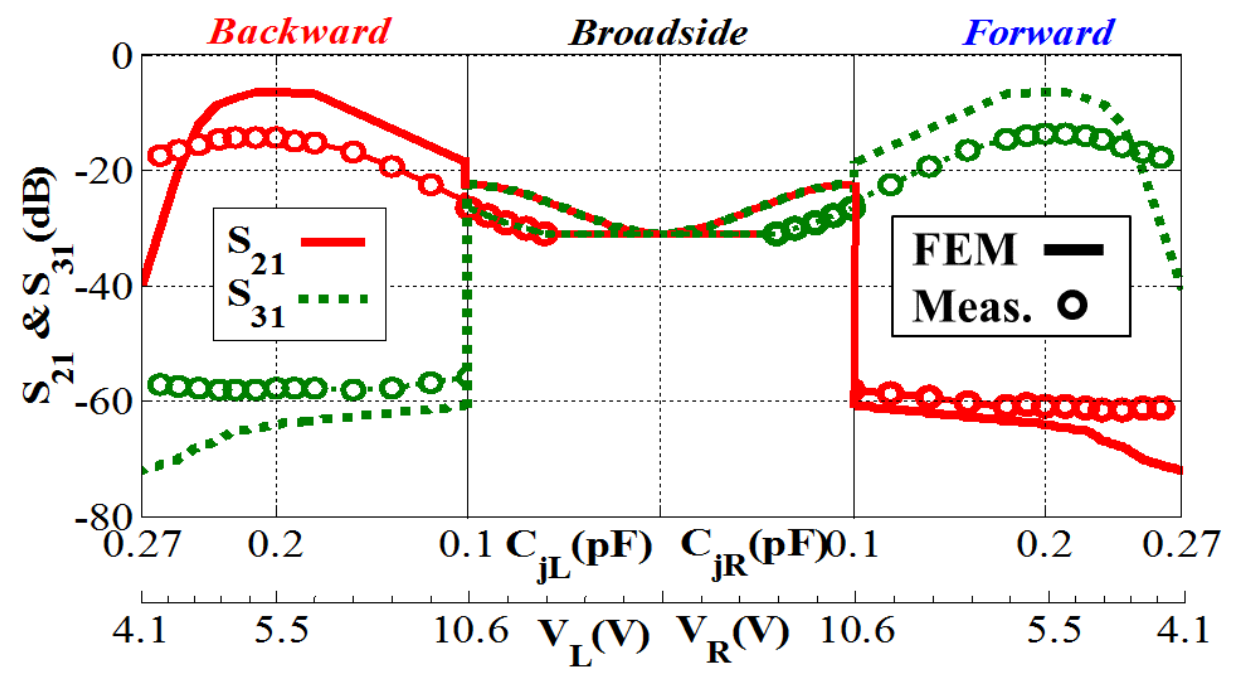

(b)

Fig. 3.5.5 Measured and simulated S-parameters for the full-space scanned 1D FP LWA at 5.5GHz. a) Input matching $S_{11}$ b) Transmission $S_{21}, S_{31}$. 
Fig. 3.5.5(b) illustrates the transmission coefficients between the input port and the two ports located at the left $\left(S_{21}\right)$ and right $\left(S_{31}\right)$ sides (see Fig. 3.5.1). As expected, when the antenna is operated in the backward scanning regime, the leaky wave is guided to the left side resulting in very low $S_{31} \approx-60 \mathrm{~dB}$ (due to the EBG regime provided by $\mathrm{HIS} \mathrm{S}_{\mathrm{RIGHT}}$ ) and $S_{21} \approx-20 \mathrm{~dB}$ (which corresponds to the energy which has not been radiated by the left-propagating leaky wave, see Fig. 3.5.5(a)). These values are interchanged in the forward-scanning configuration, showing $S_{21} \approx-60 \mathrm{~dB}$ and $S_{31} \approx-20 \mathrm{~dB}$, since the energy is guided to the right side. Finally, in the broadside regime the energy is guided to both sides presenting equal values of $S_{21}=S_{31} \approx-30 \mathrm{~dB}$. The transmission at broadside is $10 \mathrm{~dB}$ lower than in the scanning configuration due to the fact that leaky wave presents higher leakage rate at the broadside splitting condition $\left(\alpha_{3} / k_{0}>0.1\right)$ than for higher scanning angles $\alpha_{y} / k_{0}<0.1$ as shown in Fig. 3.3.1. Good agreement between experiments and simulations is observed for the transmission coefficients in the three regimes, confirming the ability of the proposed FP LWA to route the leaky-wave to the desired direction. 


\subsection{Conclusions}

A novel topology to obtain fixed-frequency electronic full-space scanning from a 1D FabryPérot (FP) LWA has been described and experimentally demonstrated, fulfilling the first objective pursued in this chapter (O5). The design is derived from the half-space electronicsteering 1D FP LWA structure presented in Chapter 2, which has been modified employing a central feeding and two independently biased tunable HIS, which give rise to two independently controlled half-space electronic-steering LWAs. Two dispersion regions are identified from the leaky-mode dispersion curves: the scanning and EBG regions. On one hand, the scanning region corresponds to the zone where the LWA is operating as leaky (which was employed by the normal half-space 1D FP LWA of Chapter 2), radiating at an angle dependent on the bias voltage of the HIS varactors. On the other hand, the EBG dispersion property of the FP cavity prevents the propagation of the TE leaky mode, so it has been employed as a routingmechanism to direct the input signal, excited from the central source, to the desired half-space electronic-steering antenna. Thus, backward (negative) angles, can be reached when left section of the LWA is operating in the scanning region and right section is tuned at EBG, whereas forward angles are scanned just in the opposite configuration. Also, broadside radiation is achieved properly exciting both tunable HIS inside the scanning region ([Lovat 2006-I]). Experimental results from a fabricated prototype operating at $5.5 \mathrm{GHz}$ show an absolute continuous scanning range from $5^{\circ}$ to $25^{\circ}$ for backward and forward scanning regimes, besides broadside radiation. Thus, it is demonstrated that continuous full-space scanning can be achieved by virtue of the tunable dispersion properties of the FP cavity and two control signals, one per section. With this conclusion, second objective of the chapter (O6) is achieved. In this way, the switching mechanism proposed in [Nguyen 2011], which needs a SPDT switch and associated control signals is avoided. It should be pointed out that the proposed EBG routing mechanism is less efficient than [Nguyen 2011], as only 50\% of the antenna aperture is used to scan the beam in each quadrant. Nevertheless, the main advantage of this EBG routing proposal compared to switching schemes is that it can be naturally extended to 2D FP LWAs, as will see in next chapter, where a 2D FP antenna is divided in several discrete sectors $\left(\mathrm{S}_{\mathrm{A}}-\mathrm{S}_{\mathrm{D}}\right)$ which are independently tuned, so that the leaky wave excited at the center of the FP cavity can be routed to any of these sectors by tuning the others to EBG regime. In this way, discrete azimuthal sectorization could be obtained, providing 2D scanning of a pencil beam (in elevation $\theta$ and azimuthal $\phi$ angles) in contrast to scanned conical beams ([Costa 2011]), and without using phased-arrays ([Rock 2009], [Devogobic 2014-II]).

The work developed in this chapter has given rise to the publication of $\mathbf{2}$ peer-review international journal paper (IJ), 1 international conference paper (IC), 1 Spanish journal 
paper (SJ) and 2 Spanish conference papers (SC). These references are copied below; they have been extracted from the complete list of publications of Section 5.3.

IJ2. R. Guzmán-Quirós, J.L. Gomez-Tornero, A.R. Weily, and Y.J. Guo, "Electronic full-space scanning with 1-D Fabry-Pérot LWA using electromagnetic band-gap," IEEE Antennas and Wireless Propagat. Lett., vol.11, no., pp.1426-1429, 2012.

IJ3. Y.J. Guo, J.L. Gomez-Tornero, R. Guzmán-Quirós, and A.R. Weily, "Reconfigurable Leaky-Wave Antennas," Forum for Electromagnetic Research Methods and Application Technologies (FERMAT), vol.1, no., pp., January 2014, available online : http://www.efermat.org/files/articles/1533721af95bd9.pdf

IC5. R. Guzmán-Quirós, J. L. Gómez Tornero, M. García-Vigueras, A.R. Weily, Y. J. Guo, "Advances in electronically reconfigurable LWAs in Fabry-Pérot and SIW technologies", 7th European Conference on Antennas and Propagation (EUCAP 2013), pp. 224-228, April 2013. (Invited contribution to a convened session about "Emerging Antenna Technologies" at EuCAP 2013)

SJ4. R. Guzmán-Quirós, M. García Vigueras, J. L. Gómez Tornero, "Escaneo Electrónico Backward-a-Forward en Antenas Leaky-Wave 1D con Excitación Central", V Jornadas de Introducción a la Investigación (UPCT), Ed. UPCT, ISBN: 1888-8356, pp.102-104, April 2012.

SC6. R. Guzmán-Quirós, J. L. Gómez-Tornero, M. García-Vigueras, A. J. Martínez-Ros, "Backward-to-Forward Electronic Scanning in Symmetrically Excited 1D Fabry-Perot LeakyWave Antennas" , XXVII URSI (International Scientific Radiocommunications Union) Symposium, Elche (Alicante), Spain, Sept. 2012.

SC9. R. Guzmán Quirós, J. L. Gómez Tornero, A. J. Martínez Ros, Andrew R. Weily, Y. J. Guo, "Novel and Simple Electronically Reconfigurable Fabry-Pérot Antennas", XXVIII URSI (International Scientific Radiocommunications Union) Symposium, Santiago de Compostela, Spain, 11-13 September 2013. (First Prize Young Scientists URSI 2013) 


\section{Chapter 4 Full-Space Electronic-Steering 2D FP LWA}

In this chapter it is presented for first time a reconfigurable two-dimensional (2D) FP LWA with simultaneous electronic steering in azimuth and elevation, operating at fixed frequency. To this aim, the author presents a novel 2D FP cavity excited from its center and comprised by a top PRS and a two-dimensional tunable HIS at the bottom (the antenna can be considered as a twodimensional extension of the full-space electronic-steering 1D FP LWA presented in Chapter 3). The HIS is divided in four independently biased sectors which control the dispersion properties of four regions inside the FP cavity. Given the x-polarized electric field generated by the excitation (an optimized stacked patch antenna), TE, TM and hybrid TE/TM leaky modes are excited across the FP cavity in the $y, x$, and oblique directions respectively. The scanning and EBG dispersion regions are analyzed for the fundamental TE and TM modes. Thus, applying the electronic-steering and EBG electronic-routing mechanisms presented in the previous chapters, it is possible to reroute the leaky waves in the FP cavity, and scan the pencil beam in a continuous way in elevation and at eight different azimuth angles (apart from broadside) depending on the chosen configuration of the sectors. Four different operating regimes are then defined: TE scanning regime, TM scanning regime, hybrid TE/TM scanning regime and broadside radiation. A prototype has been designed and manufactured to demonstrate the proof of concept. The beam can take eight discrete azimuth directions: $\phi=\left[0^{\circ}, 45^{\circ}, 90^{\circ}, 135^{\circ}, 180^{\circ}\right.$, $225^{\circ}, 270^{\circ}, 315^{\circ}$. The elevational scanning range for TE and hybrid TE/TM configurations is achieved approximately from $\theta_{R A D}=+3^{\circ}$ to $\theta_{R A D}=+20^{\circ}$, and from $\theta_{R A D}=+3^{\circ}$ to $\theta_{R A D}=+12^{\circ}$ for $\mathrm{TM}$ 
configurations. Simulations and experimental results are shown and discussed. Therefore, the last specific objective of this thesis is pursued:

O7. Design of a 2D FP LWA with simultaneous and independent electronic-steering in azimuth and elevation employing a tunable HIS and the EBG electronic-routing mechanism.

This chapter is organized as follows:

Section 4.1 gives an overview on 2D full-space scanning antennas. Later, in Section 4.2 it is introduced the proposed structure of the electronic-steering 2D FP LWA. Section 4.3 shows the modal dispersion analysis performed for the initial design of the 2D FP cavity. From this analysis, the scanning and EBG regions are identified for TE and TM modes, and the operating modes of the antenna are defined. Preliminary simulated results of the antenna are also reported in this section. In Section 4.4 it is shown the fabricated prototype and it is discussed the experimental results obtained. A brief summary of the principal results and discussion on the advantages and disadvantages of this type of antennas are given in the final conclusions in Section 4.5. 


\subsection{Introduction}

Electronically-steerable 1D LWAs previously discussed are line-source antennas. This means that they can scan a fan beam in elevation (or azimuth if they are rotated) at a fixed frequency, with a beamwidth narrow in one plane, but wide in the orthogonal one. As aforementioned, this capacity is found useful for many applications in communications or exploratory RF sensing systems (e.g. indoor wireless communications, localization, medical imaging, radio astronomy, etc.). However, two-dimensional (2D) beam-scanning is also much appreciated for other applications which require of high-gain antennas with an additional reconfigurable degree of freedom (e.g. satellite based communication systems, 3D radars, tracking, connection maintenance, etc.). For example, omnidirectional antennas used in many of today's wireless devices cause interference, lowering signal quality, reducing data rates, and limiting bandwidth. Thus, a need exists for an antenna system having a narrow beamwidth to reduce interference, while also offering the flexibility of full-space scanning ([Nguyen 2007]).

Recently, in the spatial industry, two-dimensional (2D) active phased arrays have been employed to steer the coverage of a satellite electronically without physical reorientation ([Washington 2002], [Christodoulou 2012]). However, as was mentioned in the introduction of this thesis, phased arrays have several drawbacks, such as losses due to phase-shifters or circulators, besides the complexity of the feeding network, which in the case of $2 \mathrm{D}$ arrays are issues which become much more risky given the high number of elements employed, making these systems much more expensive. Thus, other solutions have been proposed in recent years based on simpler, more practical and compact structures which integrate these functionalities in one single device. Reconfigurable reflectarrays and transmitarrays have been presented as an immediate alternative to phased arrays for electronic two-dimensional beam-scanning and general beam-forming applications. As aforementioned in the introduction of this thesis, these antennas have the advantage of being high directive, and do not require of phase shifters, but they have the main drawback of requiring an external feed, what do not allow a compact design. In addition, reflectarrays suffer from additional issues due to feed blockage effects or diffraction at the edges of the reflecting surface which require of a study of the feeder type and position in order to not lose efficiency, apart from the typical losses due to substrates and the electronic devices integrated. For an accurate control of the scanned beam, an enormous number of control signals are required to tune each element (or groups of elements) in case of large arrays, what increase the complexity of the design. In this sense, other solutions have been proposed in order to provide more compact and reduced cost antennas with two-dimensional pencil-beam steering capability. For example, one-dimensional arrays of 1D LWAs have also been studied in order to procure such two-dimensional pencil-beam scanning capability ([Oliner 2007]). These systems 
combine elevation scanning by the natural frequency scanning of LWAs and scanning in the cross plane (in azimuth), by varying the phase difference between the successive parallel leakywave sources. Fig. 4.1.1(a) shows a generic schematic of this frequency-phase scanning architecture. The spacing between the line sources is chosen to avoid grating lobes and each LWA is excited individually by a feed system with phase-shifters to tune the desired phase difference between elements. Because of the proper length of the LWAs and the arrangement in the orthogonal direction, a pencil-beam form occurs (Fig. 4.1.1(b)) and will scan in both elevation and azimuth in a conical-scan manner ([Oliner 2007]). Several types of LWA have been proposed to create phased-arrays for 2D-scanning purposes ([Ettorre 2011], [Hu 1998, 1999, 2000-I, II], [Lamapariello 1987], [Li 2008-I, II, 2009], [Nguyen 2007], [Oliner 1988, 1990], [Rusch 2013]). These structures have some unusual virtues: essentially no cross polarization, no grating lobes, no blind spots within the scan volume, and simplicity of the structure. In addition, they should cost less than phased arrays because phase-shifters are needed in only one dimension ([Jackson 2008], [Oliner 2007]). For fixed-frequency scanning applications, frequency scanning is not applicable, being necessary to employ fixed-frequency electronic-scanning LWAs. In ([Lee 2007]), a Rotman lens and five interconnected reconfigurable CRLH microstrip LWAs are proposed to obtain two-dimensional scanning at fixed-frequency. The principal inconvenient of all these phased-array configuration is that they partly lose one of the most interesting LWA features, the simple feeding scheme.

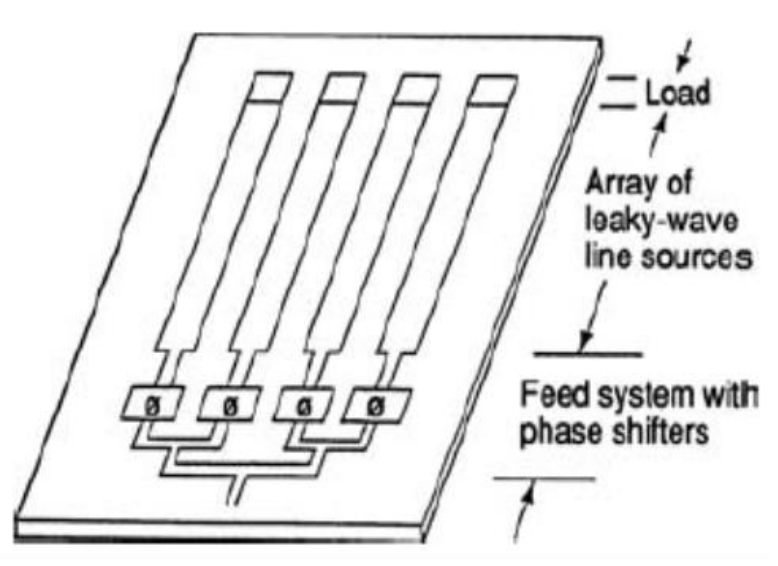

(a)

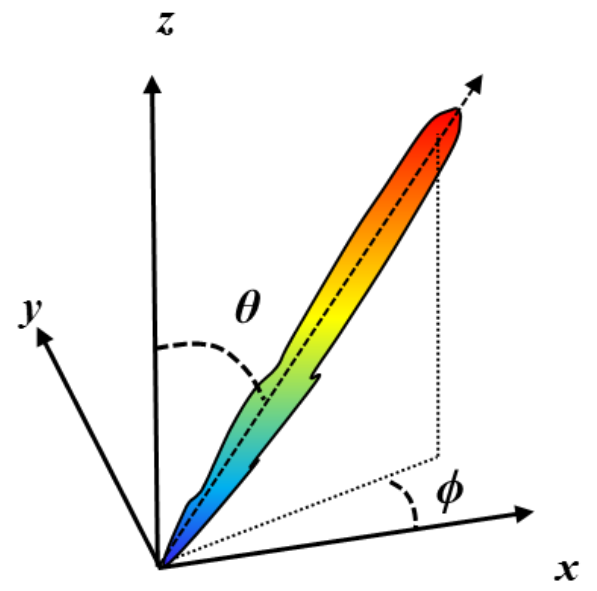

(b)

Fig. 4.1.1 (a) Schematic of the recent approach to simpler 2D scanning, involving a linear phased array of leaky-wave line sources, with frequency scanning in elevation and phase scanning in azimuth (Fig. 11.28 in [Oliner 2007]). (b) Generic pencil-beam form produced by the LWA phased array.

Other LWAs have been designed for scanning in elevation or azimuth at fixed frequency. [Chang 2012] proposed a LWA with capability for multi-plane scanning. In [Podilchak 2010] a 
pattern-reconfigurable two-dimensional LWA is considered built from a grounded dielectric substrate loaded with an annular bull-eye grating and excited by an array of four surface wave launchers (SWL). Controlling the amplitude and phase difference of the SWL array, it was demonstrated that beam-steering could be achieved at different azimuth planes, besides broadside radiation.

In this chapter we propose a more compact and simpler solution based on a single twodimensional Fabry-Pérot Leaky Wave Antenna (2D FP LWA) excited from its centre by a simple low-gain radiator. As it will be shown, this structure is able to produce a high-gain pencil beam which direction can be electronically scanned in azimuth and elevation at fixed frequency without the inconvenient of phased arrays (phase-shifters, feeding networks, reduced scalability...) and with just 4 control signals. As aforementioned in previous chapters, 2D FP LWAs were firstly introduced in 1950s by Trentiny ([Trentiny 1956]). These antennas are attractive for their properties to enhance the directivity of low-gain radiators, such as patch antennas, dipole sources, aperture antennas, etc., with a simple structure and low cost. Today, its simplicity and integrated source is still considered one of their major virtues, but the recent conception of novel designs with lower profile, enhanced directivity or enhanced bandwidth, together with the integration of new radiation characteristics such as multi-band, wideband, multiple-polarization or reconfigurable capabilities ([Boutayeb 2007], [Debogovic 2014-I], [Feresidis 2005, 2006], [Hosseini 2011], [Kelly 2007], [Krauss 2011], [Lovat 2006-II], [MateoSegura 2014], [Moghadas 2013], [Orr 2014], [Weily 2006, 2008], [Weily 2007], [Yuehe 2012], [Zhao 2005]), have made them currently one of the most promising structures to investigate for future applications. The proposed system is based on placing the low-gain antenna between a PRS and a ground plane separated at a certain distance, comprising a resonant cavity (the socalled FP cavity). Thus, the low-gain radiator turns into a source which launches a cylindrical leaky wave inside the FP cavity which propagates in the radial direction, illuminating the aperture area of the FP LWA. If the FP cavity is properly designed to satisfy the splitting condition ([Lovat 2006-I]), the antenna generates a pencil beam pointing at broadside with higher directivity, (as was observed in Fig. 2.2.8(a)). If this condition is not satisfied, (e.g. increasing the operating frequency and scanning the antenna towards endfire), then $\beta>\alpha$, and a conical beam arises (Fig. 2.2.8(b)) with the cone axis perpendicular to the aperture plane ([Jackson 2008]) because of the symmetry of the structure. Thus, the practical applicability of 2D FP LWAs can be limited for 2D scanning applications, since the beam will always evolve into a conical beam as it is scanned at higher angles, resulting in a loss of gain in the broadside direction. In the last years, it has been demonstrated that 2D FP LWAs can be used to achieve electronic scanning capabilities ([Burokur 2013], [Debogovic 2014-II], [Edalati 2011], [Ourir 2009]). In [Ourir 2009] and [Burokur 2013], a FP LWA constituted by a ground plane and a 
tunable metamaterial PRS, with a phased array as excitation source, demonstrated electronic scanning of its pencil beam in the E-plane. Also, in [Debogovik 2014-II] a similar FP LWA was proposed to scan its pencil beam in the H-plane, not employing a tunable PRS, but the phase distribution of the small phased array employed to excite the FP cavity. Also, a tunable PRS with varactors was incorporated to this 2D FP LWA in order to achieve simultaneous control of the leakage rate of the leaky wave $(\alpha)$, and hence the beamwidth of the pencil beam. [Edalati 2011] proposed a cylindrical FP structure with a tunable PRS loaded with PIN diodes, for electronic scanning in azimuth. However, despite the good applicability of these antennas, these works do not achieve two-dimensional scanning in elevation and azimuth, but solely in one scanning plane (E- or H-plane). As aforementioned, the author aims to present for first time a 2D FP LWA with simultaneous and independent electronic scanning in both planes. To do so, a two-dimensional tunable HIS loaded with varactors is placed at the bottom of the FP cavity, substituting the bottom ground plane. This surface is divided in four sectors independently biased, which allows to independently control the propagation of the leaky waves, launched by a punctual central source, in each section. Analogously to the proposed full-space electronicsteering 1D FP LWA, the electronic-steering and EBG electronic-routing mechanisms are applied to this structure by tuning each sector of the HIS. Thus, the initial broadside beam generated by the aperture in a natural way, can be scanned, rerouting the energy of the cylindrical leaky wave to the desired aperture section of the antenna. Thereby, the pencil beam can be scanned continuously in elevation, at the same time that eight different azimuth angles can be chosen, according to the configuration of the sectors of the tunable HIS. 


\subsection{Structure}

Fig. 4.2.1(a) shows a perspective view of the 3D model of the proposed reconfigurable 2D FP LWA. In this case, the structure consists of a 2D FP cavity made up of a top 2D patch-based PRS and a bottom 2D tunable HIS, as illustrated in Fig. 4.2.1(a) and Fig. 4.2.1(b) respectively. The structure is periodic in both $x$ and $y$ directions. These surfaces can be considered as a twodimensional version of the one-dimensional PRS and tunable HIS previously employed in Chapters 2 and 3.

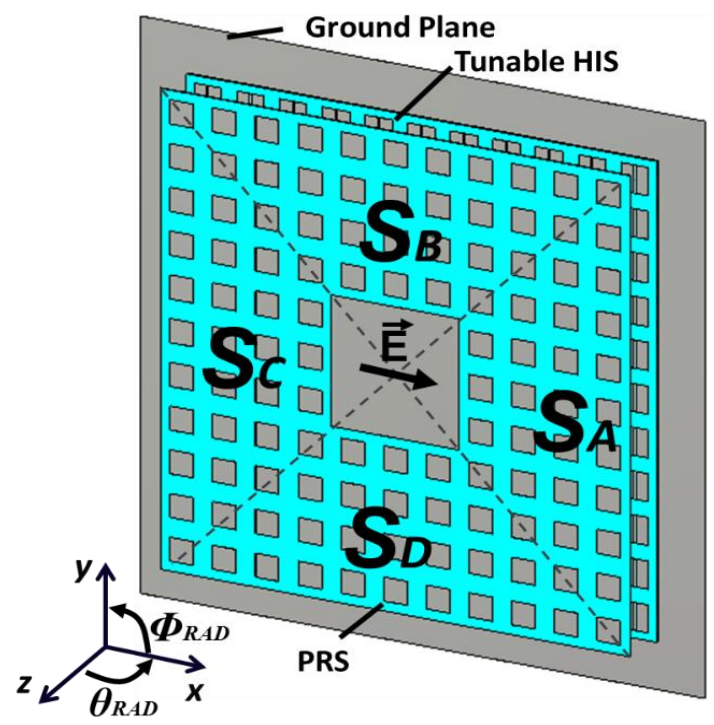

(a)

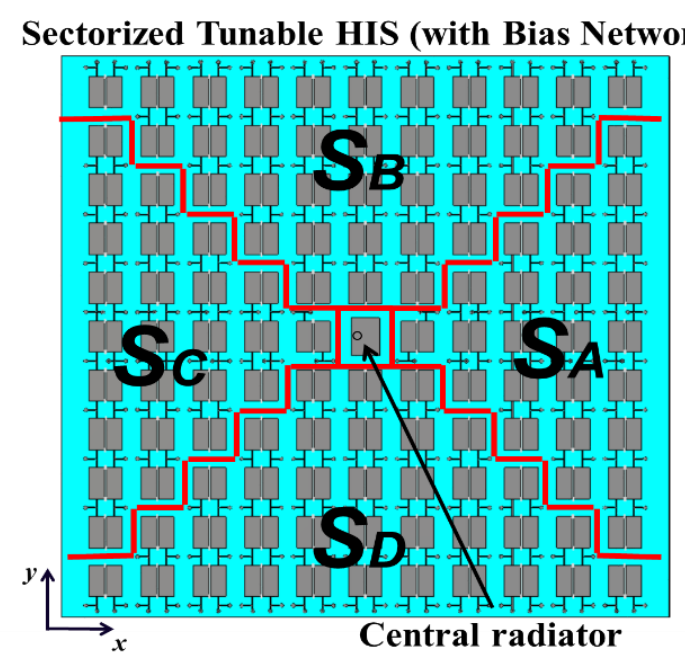

(b)

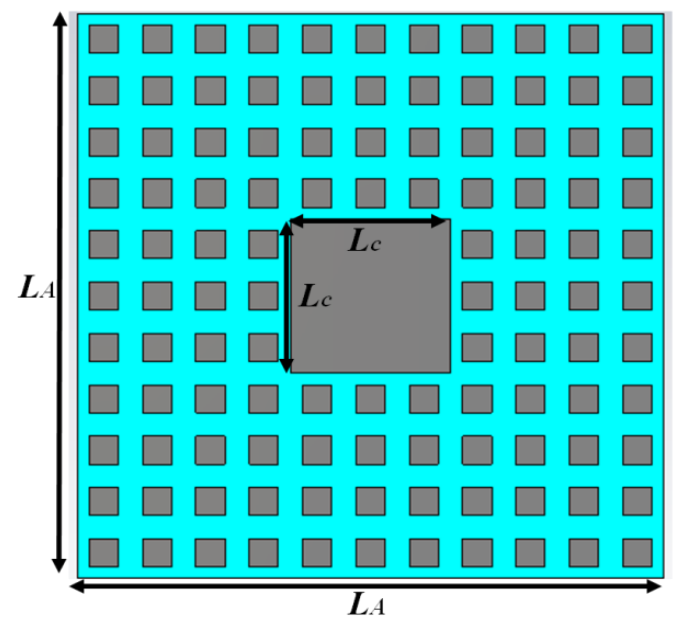

(c)

Fig. 4.2.1 (a) 3D model of the full-space electronic-steering 2D FP LWA ([CST 2010]). Antenna aperture of $A=5.4 \lambda_{0} \times 5.4 \lambda_{0}$. (b) Top view of the $2 \mathrm{D}$ tunable HIS (with bias network included) divided in four independently biased sectors $\left(\mathrm{S}_{\mathrm{A}}, \mathrm{S}_{\mathrm{B}}, \mathrm{S}_{\mathrm{C}}, \mathrm{S}_{\mathrm{D}}\right)$ and (c) Top view of the 


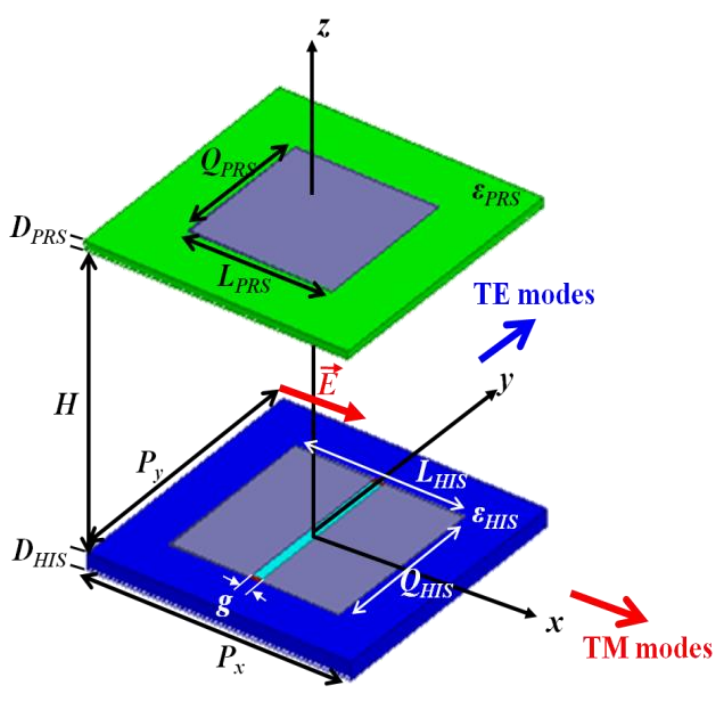

(a)

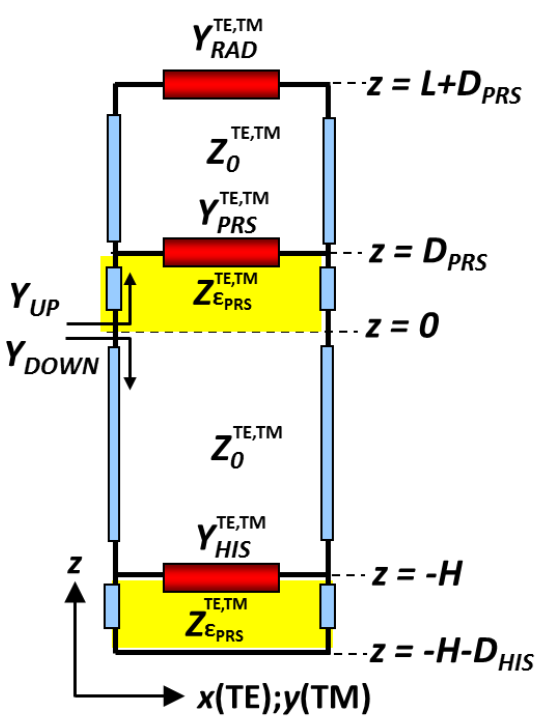

(b)

Fig. 4.2.2 (a) Single unit-cell of the periodic 2D FP structure, (b) Transverse Equivalent

Network for analyzing TE and TM leaky modes. $\left(H=27 \mathrm{~mm}, D_{H I S}=1.524 \mathrm{~mm}, \varepsilon_{r H I S}=3\right.$, $\left.P_{x}=P_{y}=26.5 \mathrm{~mm}, L_{H I S}=Q_{H I S}=17 \mathrm{~mm}, g=1 \mathrm{~mm}, L_{P R S}=Q_{P R S}=14.5 \mathrm{~mm}, D_{P R S}=0.8 \mathrm{~mm}, \varepsilon_{r P R S}=4.4\right)$.

Fig. 4.2.2(a) shows a detailed scheme with the dimensions of the PRS and HIS unit cells which compose the 2D FP LWA unit cell. It can be observed that in this case, both PRS and HIS have the same periodicity for both $x$ and $y$ axis $\left(P_{x}=P_{y}=26.5 \mathrm{~mm}\right)$. The total aperture size of the antenna is the equivalent to an array of $11 \times 11$ unit cells, creating a total aperture surface of $\mathrm{A}=5.4 \lambda_{0} \times 5.4 \lambda_{0}$ at $5.5 \mathrm{GHz}\left(294.5 \times 294.5 \mathrm{~mm}^{2}\right)$. The $2 \mathrm{D}$ tunable HIS of the antenna is constituted by 120 unit cells (see Fig. 4.2.1(b)). The single HIS unit cell (shown at the bottom of scheme depicted in Fig. 4.2.2(a)) is similar to the one used in previous 1D configurations (a square metallic patch with an air-gap in the middle loaded with two varactors). In this case, a Rogers RO4730 substrate $\left(D_{H I S}=1.542 \mathrm{~mm}, \varepsilon_{r H I S}=3, \tan \delta=0.0023\right)$ has been chosen to hold the HIS patches. The air gap where the varactors are placed still remains $g=1 \mathrm{~mm}$. The new dimensions of the patches are $Q_{H I S}=L_{H I S}=17 \mathrm{~mm}$, which have been optimized by a full-wave plane wave analysis ([CST 2010]), as explained in previous sections, to achieve the desired phase-agile response of the HIS with the desired $C_{j}$ range. The varactors have been arranged to divide the tunable HIS in four independently biased azimuthal sectors $\left(\mathrm{S}_{\mathrm{A}}-\mathrm{S}_{\mathrm{D}}\right.$, Fig. 4.2.1(b)). The original bias network taken from [Weily 2008], has been redesigned to adapt its dimensions to the new HIS and provide the input to the four DC bias signals which will control each sector separately.

Regarding the PRS, it is constituted by an array of 112 patches and a central metallic patch (Fig. 4.2.1(c)). The PRS unit cell (shown at the top of scheme depicted in Fig. 4.2.2(a)) has been slightly modified with respect to previous designs, now consisting of a square metallic patch with dimensions $Q_{P R S}=L_{P R S}=14.5 \mathrm{~mm}$. The new dimensions of the PRS patches have been 
designed in order to achieve a leakage rate which allows a high radiation efficiency ([Oliner 2007]), avoiding that too much energy arrives at the edges of the antenna and reflects back to the source ([García-Vigueras 2012-II), spoiling the radiation patterns with undesired reflected lobes. A square FR4 dielectric slab have been used to hold the PRS patches $\left(D_{P R S}=0.8 \mathrm{~mm}\right.$, $\left.\varepsilon_{r P R S}=4.4, \tan \delta=0.018\right)$. Finally notice that the square metallic patch placed at the center of the PRS (with length $L_{c} \times L_{c}=79.5 \times 79.5 \mathrm{~mm}$ ) (Fig. 4.2.1(b)) is intended to reduce the high radiation power which arises at the center of this type of $2 \mathrm{D}$ structures, where the excitation source is located and the peak electric fields are found. It was checked that this radiation can deteriorate the radiation patterns at certain configurations introducing strong secondary lobes close to broadside.

Concerning the central excitation source, a probe-fed stacked patch antenna was chosen to provide a higher degree of freedom for optimizing the input matching of the antenna ([Weily 2008]). Other simpler alternatives, such as patch antennas, or coaxial excitations, do not offer additional design parameters to provide better input matching response when the HIS is reconfigured. The lower patch is attached to an SMA connector by a coaxial probe. The lower patch can be observed at the middle of the tunable HIS in Fig. 4.2.1(b). The source will launch a cylindrical x-polarized leaky mode from the center of the FP cavity which propagates outward in the radial direction from the source. Thus, TE and TM leaky modes will propagate along the principal $y$ and $x$ directions respectively, and hybrid TE/TM modes along intermediate radial directions. The propagation of these modes will be dependent on the tuning state on which operates each one of the eight reconfigurable sectors defined. The electronic scanning of this antenna is essentially based on the same scanning mechanism and EBG electronic-routing concepts explained in previous chapters for the half-space and full-space reconfigurable 1D FP LWAs. Nevertheless, given the two-dimensional topology of this new structure, twodimensional electronic-steering control of a pencil beam will be achieved, allowing discrete beam-steering in azimuth to eight different directions, besides continuous elevation scanning operating the sectors inside the scanning range of the leaky mode, as was explained for the halfspace 1D FP LWA. In order to analyze the operating points in which each sector must be tuned to achieve the different operating modes of the antenna, next subsection shows the dispersion analysis performed for studying the TE and TM leaky modes in order to identify the operating points ( $C_{j}$ ranges) which define the scanning and EBG regions for each one. 


\subsection{Dispersion Regions and Operating Regimes}

Given the centrally-excited $x$-polarized two-dimensional structure described in previous section, the TM leaky mode will propagate across sectors $S_{A}$ and $S_{C}$, whereas the TE leaky mode propagates across orthogonal sectors, $\mathrm{S}_{\mathrm{B}}$ and $\mathrm{S}_{\mathrm{D}}(y$ axis). Also note that hybrid TE/TM leaky modes are considered propagating through the diagonal directions ([Ip 1990]) of the antenna, however, their response can be extrapolated from the TE and TM leaky-mode dispersion curves, so the analysis of just the TE and TM leaky modes will be sufficient for estimating the response of the antenna. To this aim, a dispersion analysis have been performed by the TEN shown in Fig. 4.2.2(b), which admittances and impedance characteristics have been analyzed for TE or TM incidence depending on whether TE or TM leaky mode is analyzed, according to the coordinate system described (see Fig. 4.2.2(a)). Highlight that in this case, to obtain the equivalent admittances of the PRS and the HIS, the characterization process has been performed separately for TE and for TM incidence. Also note that the TEN represents a one-dimensional approach, which means that the TE or TM modes are studied as they were propagated through an infinite 1D LWAs extended along the longitudinal $y$ or $x$ direction, supporting a TE or a TM leaky mode respectively. The same approach was successfully employed in [García-Vigueras 2012-II] for designing passive (non-reconfigurable) double-layer 2D FP LWAs.

Fig. 4.3.1 shows the dispersion curves obtained for the TE and TM leaky mode for the structure presented in previous section. As occurred for the full-space 1D FP LWA of the previous section, a scanning region and an EBG region can be identified for both TE and TM leaky modes. Thus, the scanning region for the TE leaky mode theoretically extends in the range $C_{j}=[0.15 \mathrm{pF}, 0.25 \mathrm{pF}]$, according to the TRM predictions, whereas for the TM leaky mode is defined in the range $C_{j}=[0.17 \mathrm{pF}, 0.25 \mathrm{pF}]$. On the other hand, both TE and TM leaky modes possess an EBG region which extends from $C_{j}>0.25 \mathrm{pF}$ to beyond $C_{j}=0.3 \mathrm{pF}$. Both TE and TM leaky-mode dispersion curves obtained in Fig. 4.3.1 will be considered as a first approach to design this reconfigurable antenna and to know the operating regimes of each mode. Extrapolating the hypothesis demonstrated from the full-space 1D LWA to the 2D case, the energy injected by the central source will be routed towards a given sector by tuning properly the desired sectors of the antenna into the scanning region while the rest of the sectors are tuned into the EBG region. Therefore, it will be demonstrated how a pencil beam can be continuously scanned in the elevation plane $\left(\theta_{R A D}\right)$ and also keeping different discrete azimuthal angles $(\phi)$ according to the illuminated sectors. 


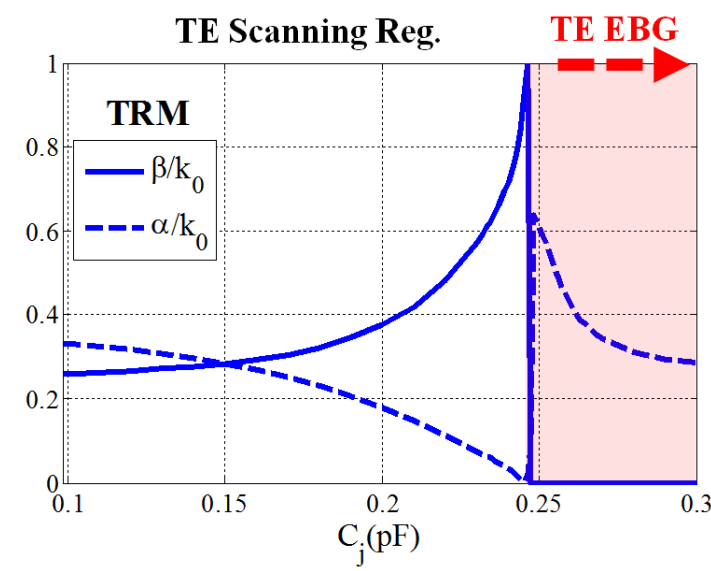

(a)

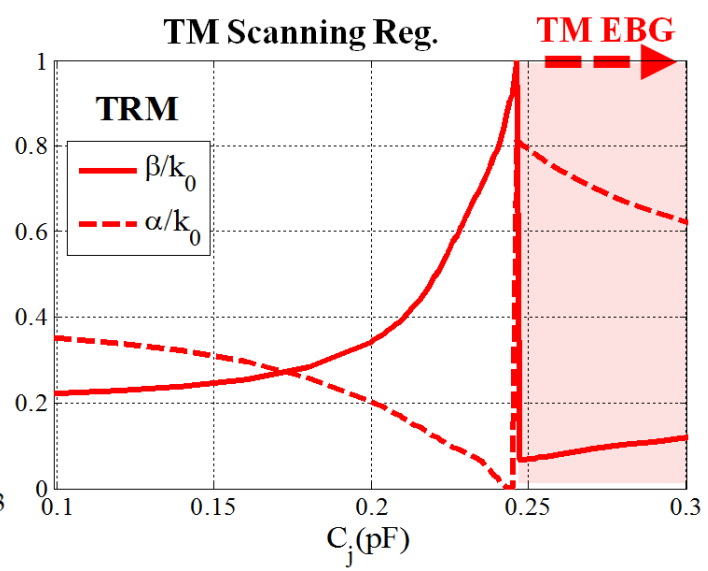

(b)

Fig. 4.3.1 (a) TE and (b) TM dispersion curves as function of $C_{j}$ at $5.5 \mathrm{GHz}$.

As a result of combining the operation of different sectors, four operating regimes can be defined for the antenna:

A. TM operating regime (radiation by TM leaky mode): TM leaky mode propagating along the $\pm x$ axis. $S_{A}$ or $S_{C}$ is active (tuned to the scanning region) while the other three sectors are inactive (tuned to the EBG region).

B. TE operating regime (radiation by TE leaky mode): TE leaky mode propagating along the $\pm y$ axis. $S_{B}$ or $S_{D}$ is active and all other sectors are inactive.

C. Hybrid operating regime (radiation by hybrid TE/TM leaky mode): Hybrid leaky mode propagating along the diagonal directions when two adjacent sectors are active (possible configurations: $S_{A} \& S_{B}, S_{B} \& S_{C}, S_{C} \& S_{D}$ or $\left.S_{D} \& S_{A}\right)$ and the other two sectors tuned to the EBG region.

D. Broadside radiation: A pencil beam pointing at boresight can be obtained when all the sectors are active and tuned at or slightly below the splitting condition [Lovat 2006-I].

By properly using the information obtained from the TE/TM leaky-mode dispersion curves of Fig. 4.3.1, the sector can be tuned in its appropriate dispersion region to operate in one of the aforementioned four radiation regimes. To give physical insight in the operating principles which govern the reconfiguring mechanism of this structure, simulated results obtained from a 2D FP LWA analyzed in CST ([CST 2010]) are shown in Fig. 4.3.2. Fig. 4.3.2(a) and Fig. 4.3.2(b) illustrate the simulated near fields obtained inside the 2D FP cavity and the associated radiation patterns for different configurations of the sectors respectively (in U-V coordinates). From the fields depicted in Fig. 4.3.2(a), it is observed how the antenna aperture field 
distribution (illumination) can be modified by changing the sectors activated at EBG or scanning region, varying the azimuthal direction of the travelling leaky wave. These aperture illuminations lead to the synthesis of radiated pencil beams which can be steered to discrete azimuthal angles $\phi=\left[0^{\circ}, 45^{\circ}, 90^{\circ}, 135^{\circ}, 180^{\circ}, 225^{\circ}, 270^{\circ}, 315^{\circ}\right]$. This is demonstrated by the position of the pencil beam in the radiation patterns obtained in Fig. 4.3.2(b) for each configuration. Moreover, for any constant azimuthal angle $\phi$, continuous scanning of the elevation angle can be achieved in the range $\theta_{R A D}=\left[5^{\circ}, 25^{\circ}\right]$ approximately, by properly tuning the active sector inside the scanning range. Finally, optimal broadside beam is obtained (Fig. 4.3.2(b)) when the four sectors are tuned to accomplish the splitting condition ([Lovat 2006-I]), resulting in a cylindrical leaky wave propagating outward in the radial direction ([Ip 1990]) (Fig. 4.3.2(a)). Note that TE and TM leaky modes have a very similar phase constant $\left(\beta_{x} \approx \beta_{y}\right)$ in this case (as observed from Fig. 4.3.1).

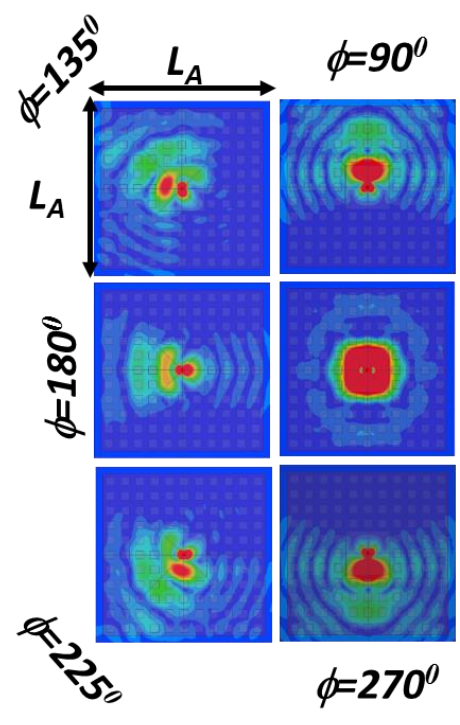

(a)

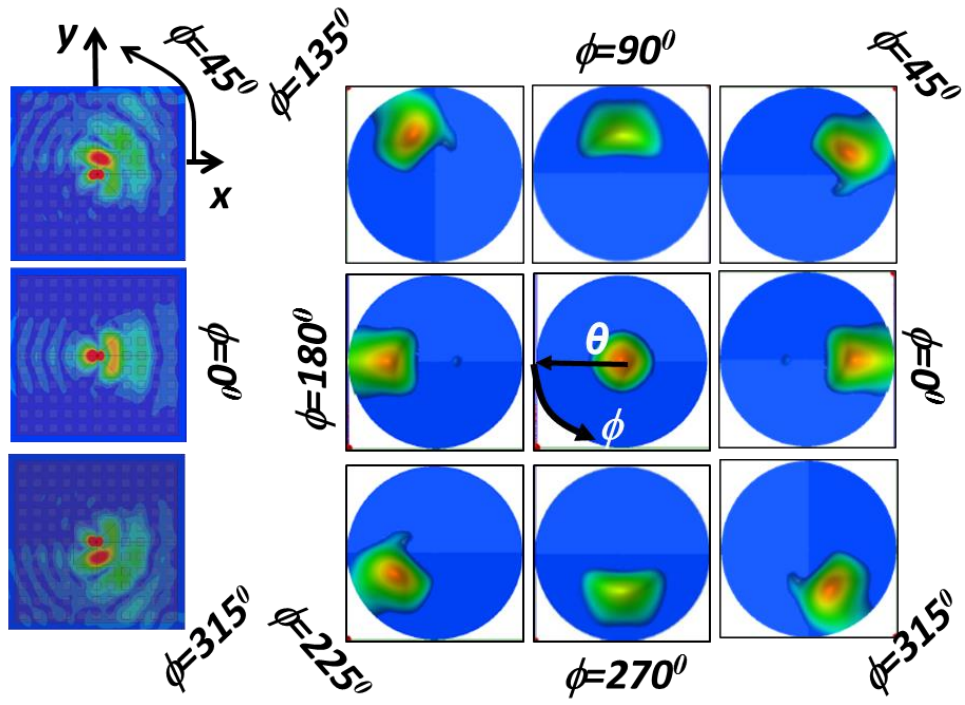

(b)

Fig. 4.3.2 (a) Near fields inside the 2D FP cavity $(z=H / 2)$ for several configurations of the sectors and (b) Associated radiation pattern in U-V coordinates (represented elevation $(\theta)$ range

$$
\left.\theta=\left[0^{\circ}, 45^{\circ}\right]\right) .
$$

Radiation patterns in polar coordinates for some representative operating configurations are depicted in Fig. 4.3.3. Each case is represented in its principal elevational plane in order to show the variation of the pencil beam scanning angle in elevation. It must be kept in mind that symmetrical scanning response is expected for the symmetrical TE, TM and Hybrid configurations, given the perfect symmetry of the structure simulated (as was shown in Fig. 4.3.2). From these results, it should be mentioned that the EBG operating point chosen to tune any sector (TE or TM) into the stop band region has been chosen $C_{j}=1 \mathrm{pF}$. The election of this point comes from a previous analysis which was performed to analyze the effect of the EBG operating point on the performance of the antenna (in terms of maximum directivity and lower 
secondary lobe level). This study demonstrated that better response was obtained as $C_{j}$ increased, so it has been adopted the maximum value attained by the varactors' dynamic range.

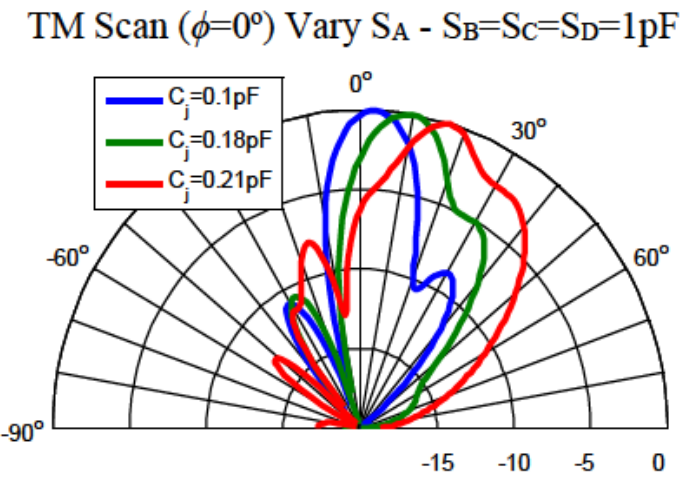

(a)

Hybrid Scan $\left(\phi=0^{\circ}\right)$ Vary $\mathrm{S}_{\mathrm{A}}=\mathrm{S}_{\mathrm{B}}-\mathrm{S}_{\mathrm{C}}=\mathrm{S}_{\mathrm{D}}=1 \mathrm{pF}$

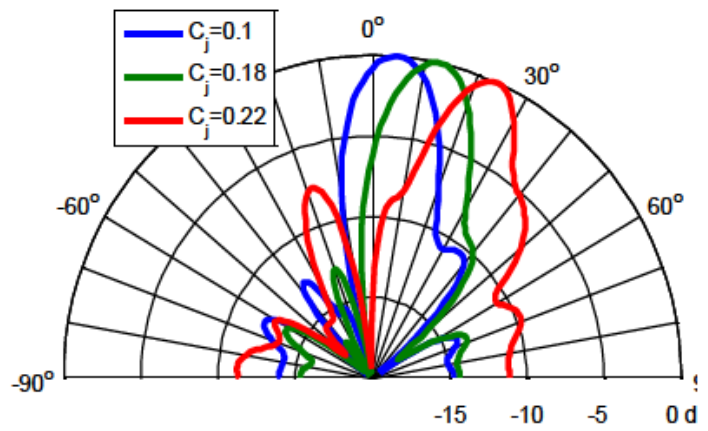

(c)

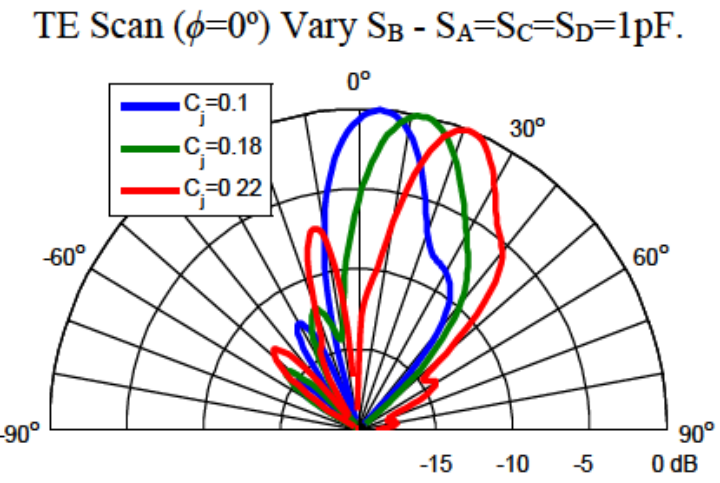

(b)

Broadside Radiation $\mathrm{S}_{\mathrm{A}}=\mathrm{S}_{\mathrm{B}}=\mathrm{S}_{\mathrm{C}}=\mathrm{S}_{\mathrm{D}}=0.1 \mathrm{pF}$.

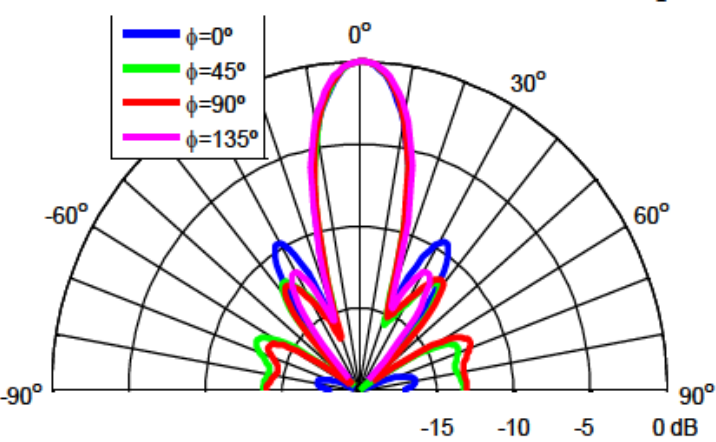

(d)

Fig. 4.3.3 (a) Normalized radiation patterns at 5.5 GHz obtained from simulations (FEM ([CST 2010])). (a) TM leaky-mode scan (E-plane) at $\phi=0^{\circ}$, (b) TE leaky-mode scan (H-plane) at $\phi=90^{\circ}$, (c) Hybrid leaky-mode scan (EH-plane) at $\phi=45^{\circ}$, (d) Broadside radiation (four cutting planes).

As observed from the radiation patterns shown in Fig. 4.3.3(a), a single beam is scanned when the antenna is operating at the TM scanning regime, achieving a scanning range $\theta_{R A D}=\left[3^{\circ}, 19^{\circ}\right]$ for azimuth angles $\phi=\left[0^{\circ}, 180^{\circ}\right]$. In Fig. 4.3.3(b), the antenna is configured to point at $\phi=0^{\circ}\left(\mathrm{S}_{\mathrm{A}}\right.$ active, $\mathrm{S}_{\mathrm{B}}=\mathrm{S}_{\mathrm{C}}=\mathrm{S}_{\mathrm{D}}=1 \mathrm{pF}$ ). Also, the TE regime achieves a similar response: $\theta_{R A D}=\left[4^{\circ}, 21^{\circ}\right]$ (Fig. 4.3.3(a)), for $\phi=\left[90^{\circ}, 270^{\circ}\right]$. In Fig. 4.3.3(b), the antenna is configured to point at $\phi=90^{\circ}\left(\mathrm{S}_{\mathrm{B}}\right.$ active, $\mathrm{S}_{\mathrm{A}}=\mathrm{S}_{\mathrm{C}}=\mathrm{S}_{\mathrm{D}}=1 \mathrm{pF}$ ). The hybrid regime achieves a slightly larger scanning range than the others, obtaining a scanning range $\theta_{R A D}=\left[5^{\circ}, 25^{\circ}\right]$ for $\phi=\left[45^{\circ}, 135^{\circ}, 225^{\circ}, 315^{\circ}\right]$. In this case, Fig. 4.3.3(b) presents the pattern when the antenna is configured to point at $\phi=45^{\circ}\left(\mathrm{S}_{\mathrm{A}}=\mathrm{S}_{\mathrm{B}}\right.$ active, $\mathrm{S}_{\mathrm{C}}=\mathrm{S}_{\mathrm{D}}=1 \mathrm{pF}$ ). As observed, similar scanning range response is obtained from simulations for every operating regime, as was expected from the design process. Finally, in Fig. 4.3.3(d) it is shown a radiation pattern for broadside configuration. In this case, the four sectors of the HIS are allowing propagation of the leaky wave, so the whole aperture is illuminated (see Fig. 4.3.3(a)), achieving a directive beam pointing at broadside if the sectors are properly tuned at 
the optimum operating point. In this case, simulations predict optimum broadside radiation (in terms of maximum directivity) when all sectors are tuned at $C_{j}=0.1 \mathrm{pF}$, which should correspond to the point where the splitting condition is supposed to be satisfied. It can be observed that this operating point is lower than the predicted by the TRM, that for the TE mode, in Fig. 4.3.1(a), is close to $C_{j}=0.15 \mathrm{pF}$, and for the TM, Fig. 4.3.1(b), close to $C_{j}=0.17 \mathrm{pF}$. These discrepancies can be attributed to errors between the TEN approach and the real finite model of the 2D FP LWA, which is excited by a real source in simulations (a stacked patch antenna).

From these simulated results, it is clearly demonstrated that continuous electronic elevation scanning at eight discrete azimuthal directions can be achieved applying the previously described dispersion-engineering techniques to a 2D FP structure. In next sections, a fabricated prototype is tested to confirm experimentally these results. 


\subsection{Experiments}

\subsubsection{Fabricated Prototype}

Fig. 4.4.1 shows photographs of the manufactured tunable HIS with varactors and the PRS which constitute the 2D FP LWA. The manufacture process as well as the experimental tests has been developed in the facilities of CSIRO ICT center, in Sidney (Australia). Fig. 4.4.1(a) and Fig. 4.4.1(b) show the front and rear views of the HIS, respectively. Fig. 4.4.1(a) shows the 11x11 HIS unit cells which constitute the tunable HIS layer, with the bias network deployed. The bias network scheme presents a similar scheme than the one employed for the half-space 1D FP LWA. Nevertheless, in this case, four control signals are employed to tune each sector of the HIS independently. The bias networks are responsible for isolating DC source from RF and vice versa. Short-circuited stubs reflect RF currents induced on the patches, isolating the DC source, whilst the bypass capacitors isolate DC signals from via holes used for short-circuited the stubs. Same bypass capacitors employed in the half-space 1D FP LWA have been used here. However, a different varactor diode model has been employed, in contrast to the prototypes of the half-space and full-space 1D FP LWAs. In this case, it has been chosen the Aeroflex Metelics MGV 125-20-0805-2 ([Aeroflex 2005]), which has a larger $C_{j}$ tuning range from $0.09 \mathrm{pF}$ to $1 \mathrm{pF}$ for a corresponding bias voltage $\left(V_{R}\right)$ from 2 to $20 \mathrm{~V}$. As will see, this tuning range will be convenient for tuning the structure into a more proper EBG region, which requires of $C_{j}=1 \mathrm{pF}\left(V_{R}=2 \mathrm{~V}\right)$, as commented in previous section. The typical performance $V_{R}$ vs $C_{j}$ of this varactor diode is given in Fig. 4.4.2 ([Aeroflex 2005]). Same RLC model shown in Subsection 2.5.4 has been introduced in the full wave simulations to characterize the varactors' response.

Fig. 4.4.1(b) shows the HIS DC wiring. As observed, DC is connected by through via holes drilled on the metallic ground plane. These vias drive the DC signals to the patches at each respective sector from the four DC connectors. Also, front and rear views of the PRS can be seen in Fig. 4.4.1(c) and Fig. 4.4.1(d) respectively. The 120 square patches and the central patch which constitute the PRS can be observed in Fig. 4.4.1(a). A FR4 slab is employed to hold this array of patches. Notice that this surface is supported at a distance $H=27 \mathrm{~mm}$ from the HIS by 12 nylon standoffs mounted at the edges of the HIS (see Fig. 4.4.1(a)). Again, the height $H$ has been optimized firstly by the TEN, and later by full-wave simulations, to optimize the scanning range with respect to the dynamic range of the varactors. 


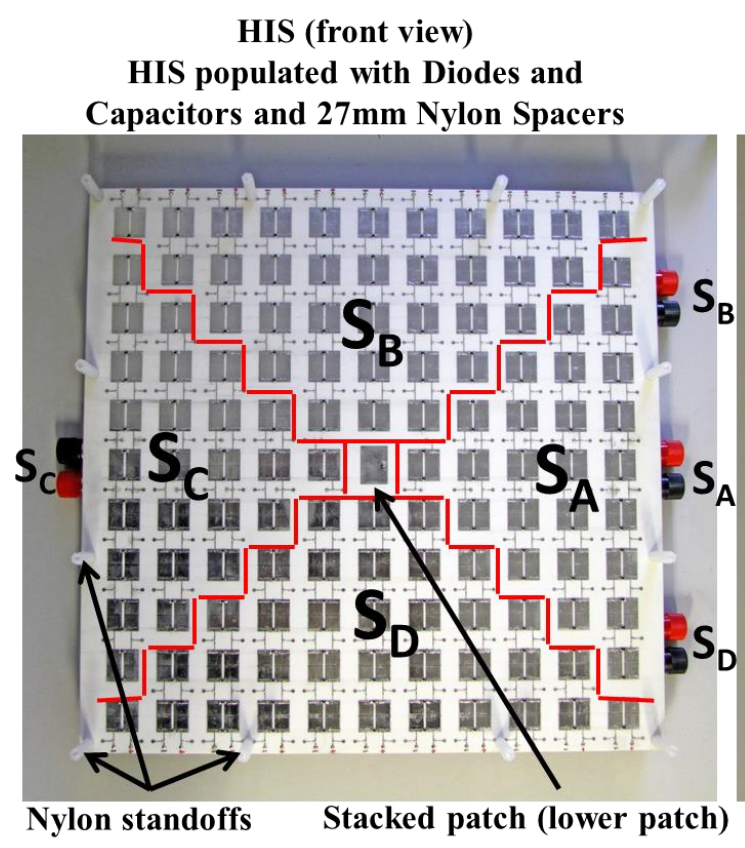

(a)

Top PRS (front view)

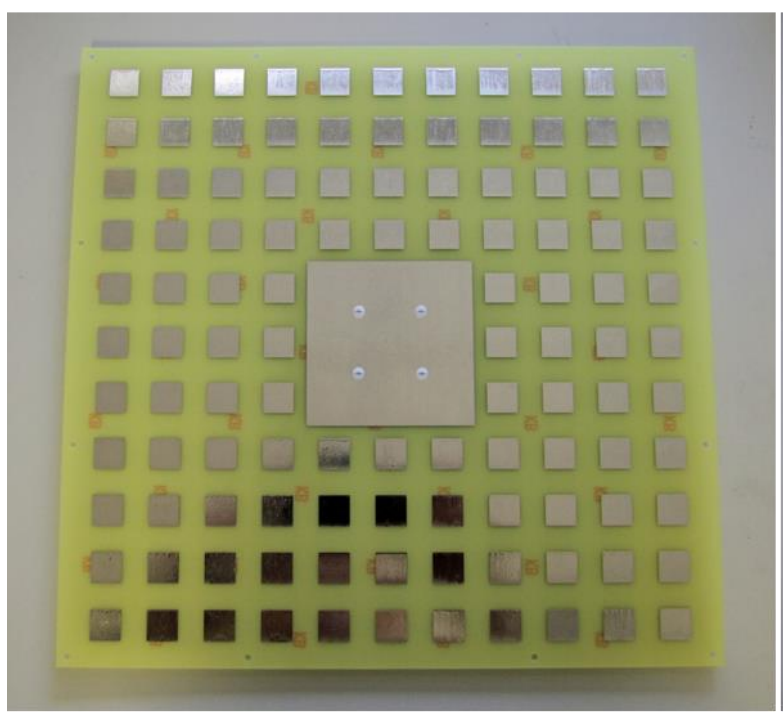

(c)
HIS (rear view) HIS DC wiring and SMA connector

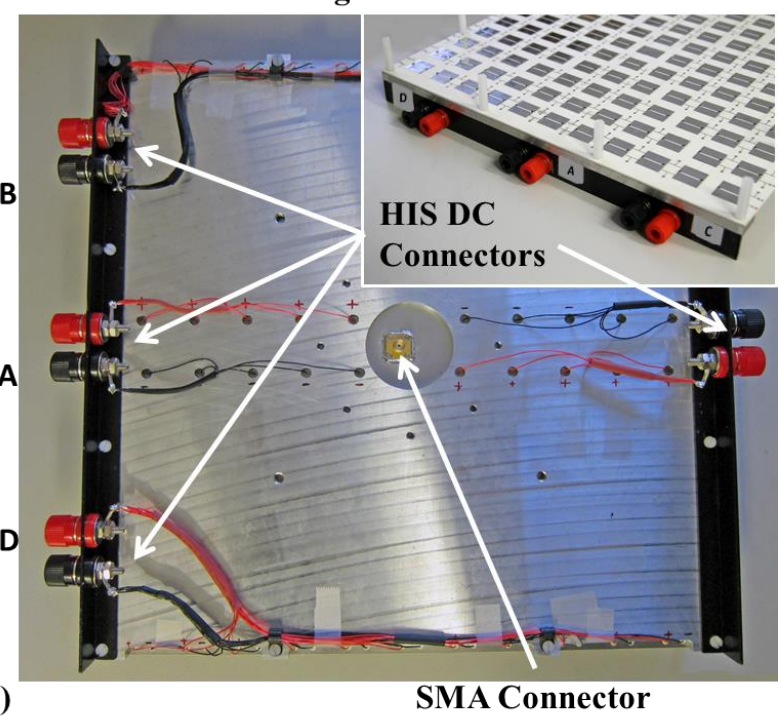

(b)

\section{Top PRS (rear view)}

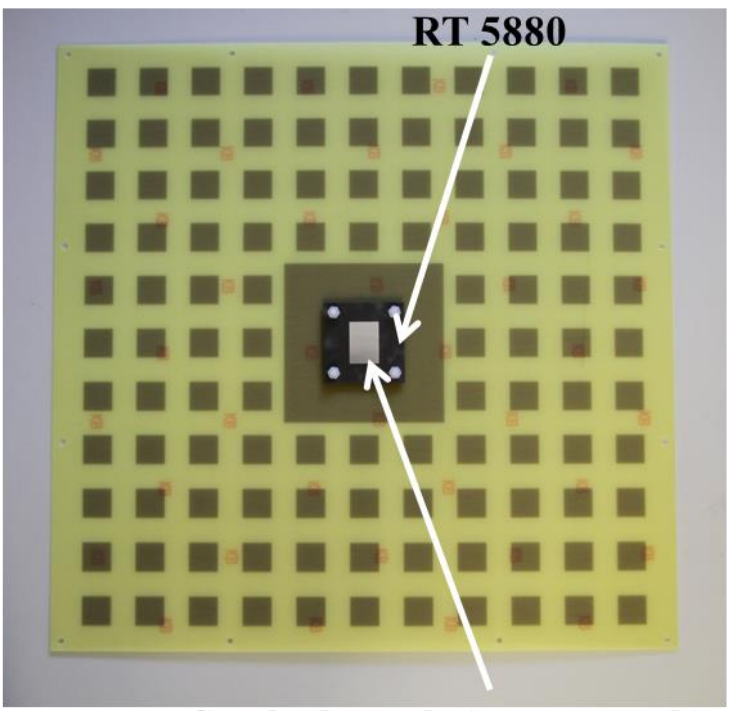

Stacked patch (upper patch)

(d)

Fig. 4.4.1 Photographs of manufactured tunable HIS: (a) front view and (b) rear view.

Photographs of the manufactured PRS: (c) front view and (d) rear view.

Regarding the central excitation, two pieces mainly compose the stacked patch antenna designed to excite the structure. Firstly, the lower patch can be found at the middle of the tunable HIS (see Fig. 4.4.1(a)), where it is attached to an SMA connector (see Fig. 4.4.1(b)) by a coaxial probe. Secondly, a parasitic patch is located over the lower patch antenna. This parasitic patch allows a better coupling of the energy into de resonating leaky modes which propagate through the structure. Hence this excitation source offers better matching for the 
reconfigurable FP cavity than single-patch antennas. Four bolts (Fig. 4.4.1(d)) drilled on the PRS at the central patch are responsible for holding the parasitic patch together with the RT Duroid 5880 slab $\left(\varepsilon_{r P R S}=4.4, \tan \delta=0.018\right)$ which holds the patch.

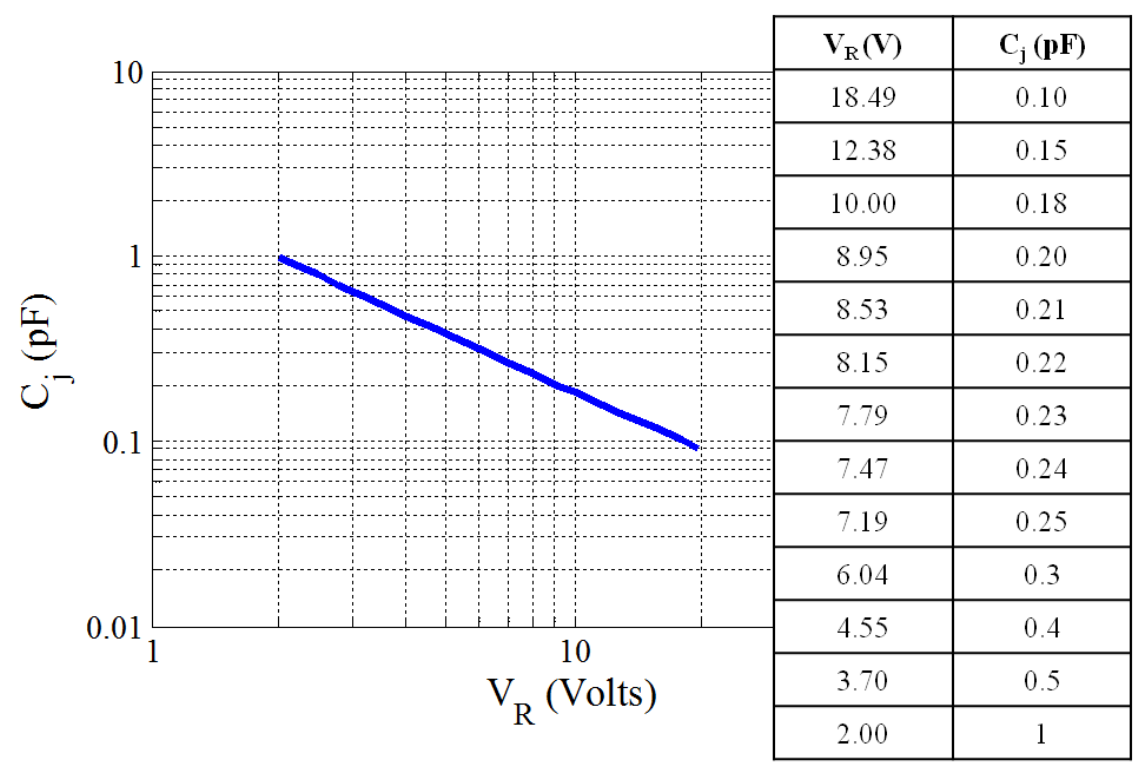

Fig. 4.4.2 Typical performance $V_{R}$ vs $C_{j}$ of Aeroflex Metelics MGV 125-20-0805-2 ([Aeroflex 2005]).

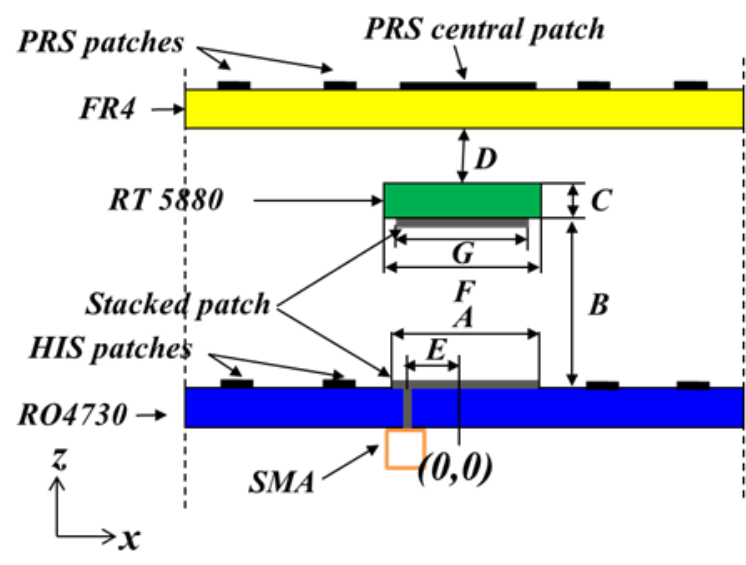

(a)

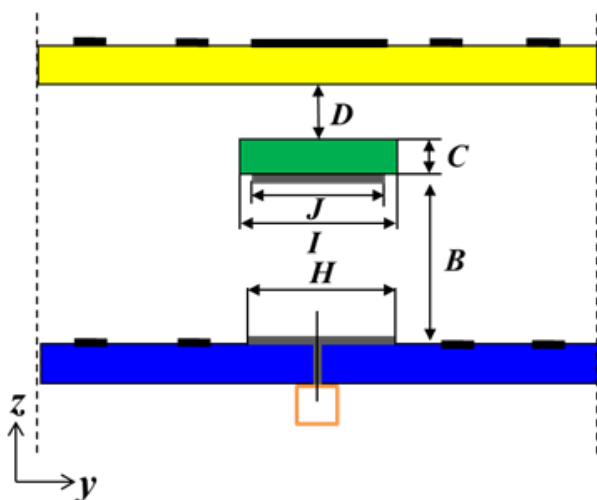

(b)

Fig. 4.4.3 Schematic of the stacked patch antenna employed for exciting the 2D FP cavity. (a)

Side view (XZ plane), (b) Side view (YZ plane).

A schematic of the stacked patch antenna designed to excite the 2D FP cavity is given in Fig. 4.4.3. The excitation source was optimized for operating at the different operating regimes of the antenna at a fixed frequency of $5.5 \mathrm{GHz}$. Again, the dynamic input impedance of this antenna, given the high number of operating regimes in which the reconfigurable 2D FP LWA can operate (6 more than for the full-space 1D FP LWA), hindered the design process of the source, which must achieve acceptable S11 for every operating regime. The stacked patch 
antenna offers a higher degree of freedom than other solutions, such as the single patch or a dipole antenna, since it allows to play with additional physical parameters related to the parasitic patch. Again, full-wave simulations were performed to optimize these dimensions. However, in order to avoid multiple and expensive (in terms of computational time) simulations of the 11x11 2D FP LWA, firstly a stacked patch antenna in absence of FP cavity was optimized, and later was introduced in a short 2D FP cavity of $5 \times 5$ unit cells in order to optimize the dimensions taking into account the effect of the tunable HIS patches around the lower patch, and also the effect of the top PRS in close proximity to the parasitic patch. In this scenario, the source was optimized for different extreme operating points for each TE, TM, and hybrid regime. The dimensions of the optimized source are given in Table 4.4.1.

\begin{tabular}{|c|c|c|c|}
\hline PARAM. & DIM (mm) & PARAM. & DIM (mm) \\
\hline A & 14.5 & F & 40 \\
\hline B & 25 & G & 13 \\
\hline C & $0.254\left(\varepsilon_{\mathrm{r}}=2.2\right)$ & H & 20.5 \\
\hline D & 1.746 & I & 40 \\
\hline E & 5.3 & J & 13 \\
\hline
\end{tabular}

Table 4.4.1. Dimensions of the optimized stacked-patch antenna to excite the reconfigurable 2D FP LWA. 


\subsubsection{Experimental Results}

Experimental tests of the $\mathrm{S}$ parameters and radiation patterns were performed for multiple configurations of the 2D FP LWA for different operating regimes. Some photographs taken during the test processes are shown in Fig. 4.4.4.

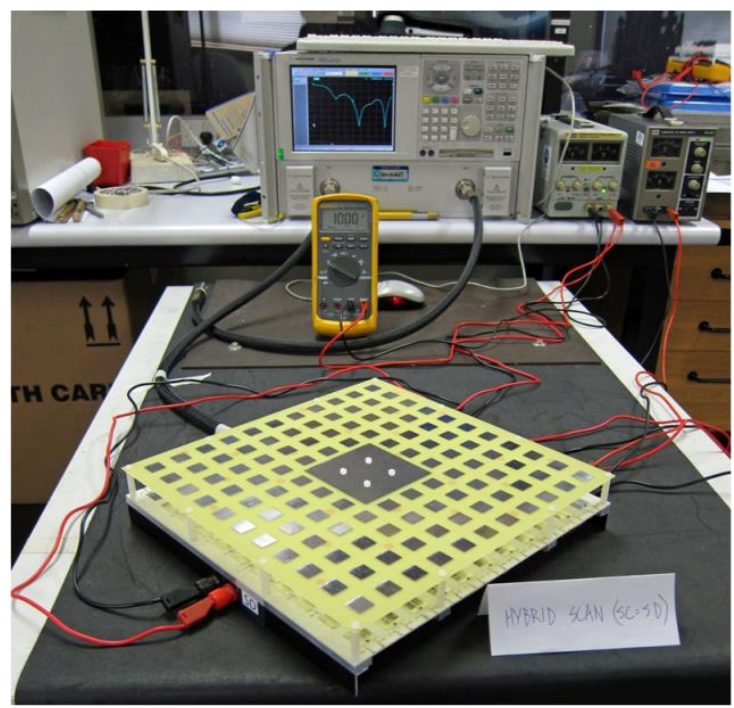

(a)

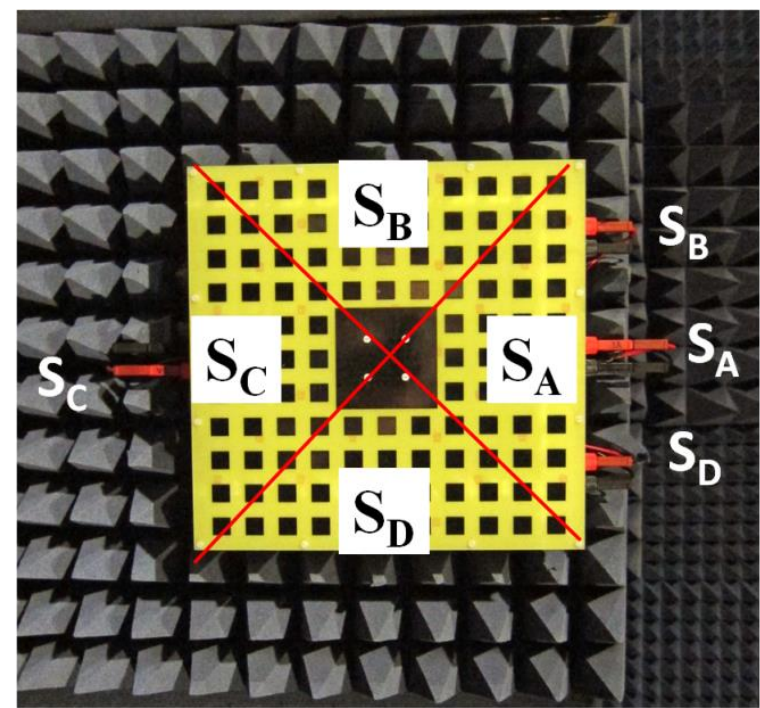

(b)

Fig. 4.4.4 Photographs of (a) S parameters measurement set-up and (b) Radiation pattern measurements set-up.

Next, the measured gain patterns for the manufactured prototype operating at TE, TM, hybrid and broadside configurations are plotted in Fig. 4.4.5. Fig. 4.4.5(a) and (b) illustrate the $X Z$ cutting plane of the pencil beam created for different TM operating modes pointed at $\phi=180^{\circ}$ and $\phi=0^{\circ}$ respectively. Continuous electronic elevation scanning range is observed. A single main beam is scanned from $\theta_{R A D}=1.5^{\circ}$ to $\theta_{R A D}=12^{\circ}$ at $\phi=0^{\circ}$, when $\mathrm{S}_{\mathrm{A}}$ is tuned from $S_{A}=18.49 \mathrm{~V}$ $\left(C_{j}=0.1 \mathrm{pF}\right)$ to $S_{A}=8.15 \mathrm{~V}\left(C_{j}=0.22 \mathrm{pF}\right)$, while the other sectors $\left(\mathrm{S}_{\mathrm{B}}, \mathrm{S}_{\mathrm{C}}, \mathrm{S}_{\mathrm{D}}\right)$ are fixed at $S_{B}=S_{C}=S_{D}=2 \mathrm{~V}\left(C_{j}=1 \mathrm{pF}\right)$. On the other hand, a similar scanning response from $\theta_{R A D}=2.5^{\circ}$ to $\theta_{R A D}=12^{\circ}$ is obtained at $\phi=180^{\circ}$ if $S_{C}$ is tuned from $S_{C}=18.49 \mathrm{~V} \quad\left(C_{j}=0.1 \mathrm{pF}\right)$ to $S_{C}=8.15 \mathrm{~V}$ $\left(C_{j}=0.22 \mathrm{pF}\right)$, while the other sectors $\left(S_{A}, S_{B}, S_{D}\right)$ are fixed at $S_{A}=S_{B}=S_{D}=2 \mathrm{~V}\left(C_{j}=1 \mathrm{pF}\right)$. Fig. 4.4.5(c)-(d) shows the scanning response for TE regime configurations. In this case, it is observed that the pencil beam is scanned from $\theta_{R A D}=3^{\circ}$ to $\theta_{R A D}=23.5^{\circ}$ for both azimuth angles $\phi=\left[90^{\circ}, 270^{\circ}\right]$. This scanning response was obtained tuning $S_{B}$ or $S_{D}$ (if it is desired pointing at $\phi=90^{\circ}$ or $\phi=270^{\circ}$ respectively) from $S_{B, D}=18.49 \mathrm{~V}\left(C_{j}=0.1 \mathrm{pF}\right)$ to $S_{B, D}=8.15 \mathrm{~V}\left(C_{j}=0.22 \mathrm{pF}\right)$, and keeping the other sectors tuned into the EBG region $(2 \mathrm{~V})$. 


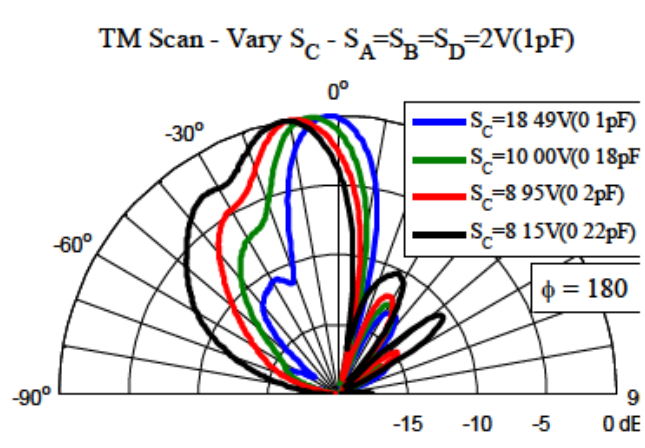

(a)

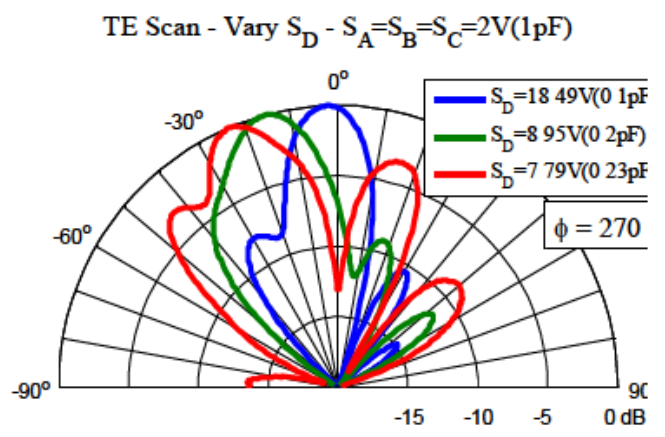

(c)

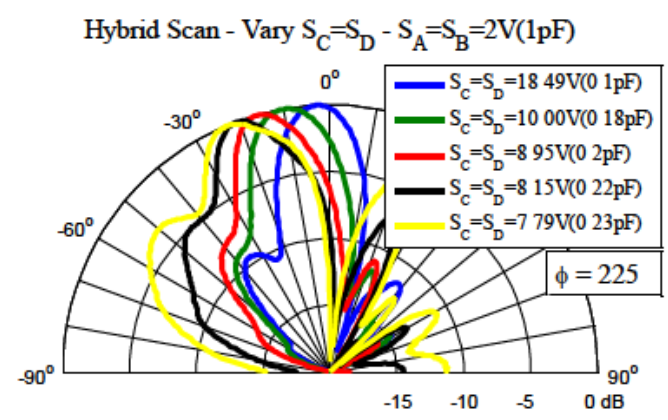

(e)

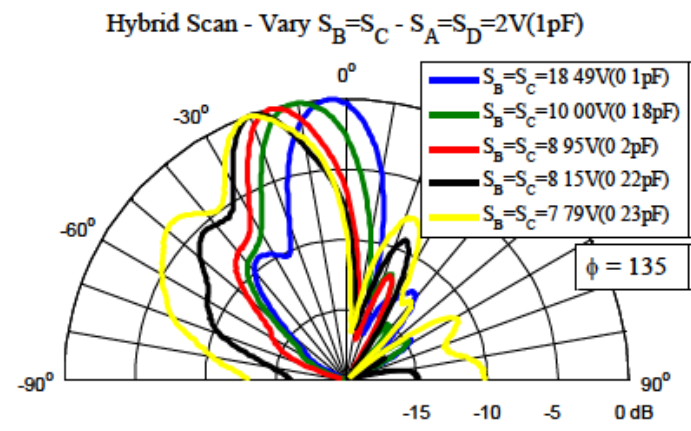

(g)
TM Scan - Vary $\mathrm{S}_{\mathrm{A}}-\mathrm{S}_{\mathrm{B}}=\mathrm{S}_{\mathrm{C}}=\mathrm{S}_{\mathrm{D}}=2 \mathrm{~V}(1 \mathrm{pF})$

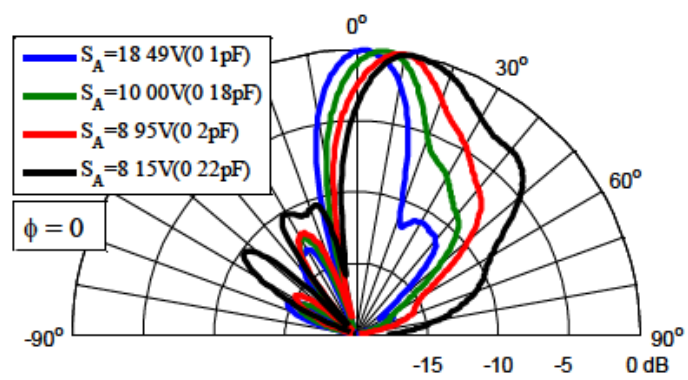

(b)

TE Scan - Vary $\mathrm{S}_{\mathrm{B}}-\mathrm{S}_{\mathrm{A}}=\mathrm{S}_{\mathrm{C}}=\mathrm{S}_{\mathrm{D}}=2 \mathrm{~V}(1 \mathrm{pF})$

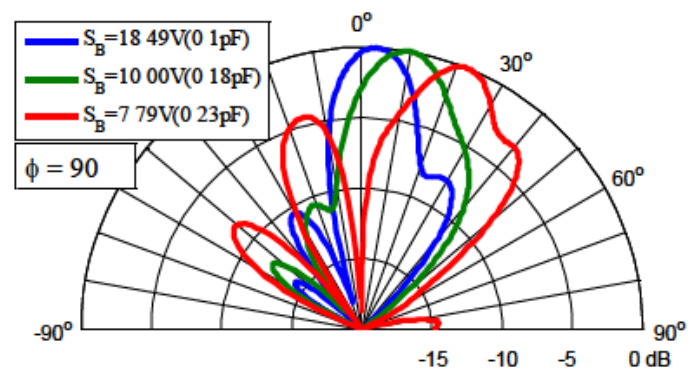

(d)

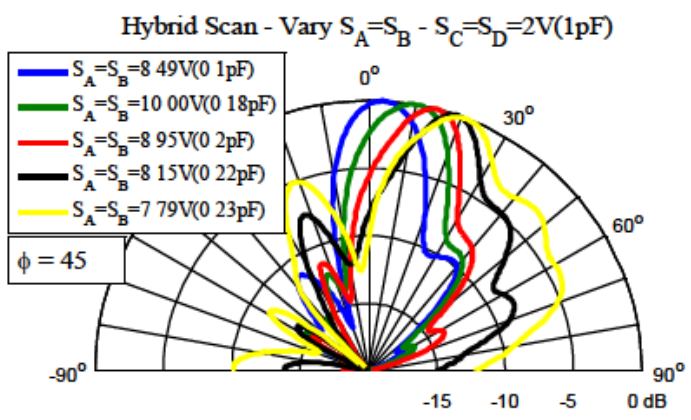

(f)

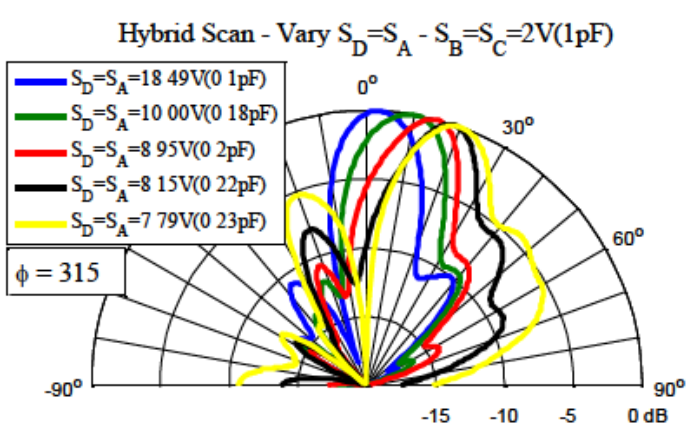

(h)

Fig. 4.4.5 Measured radiation patterns (normalized) obtained from the reconfigurable 2D FP LWA prototype at $5.5 \mathrm{GHz}$ for TM scan configurations at (a) $\phi=180^{\circ}$, (b) $\phi=0^{\circ}$ (E-Plane, $X Z$ ), for TE scan configurations at (c) $\phi=270^{\circ}$, (d) $\phi=90^{\circ}$ (H-Plane, YZ), for Hybrid TE/TM scan configurations at (e) $\phi=225^{\circ}$, (f) $\phi=245^{\circ}$, (g) $\phi=135^{\circ}$, (h) $\phi=315^{\circ}$ (diagonal planes). 


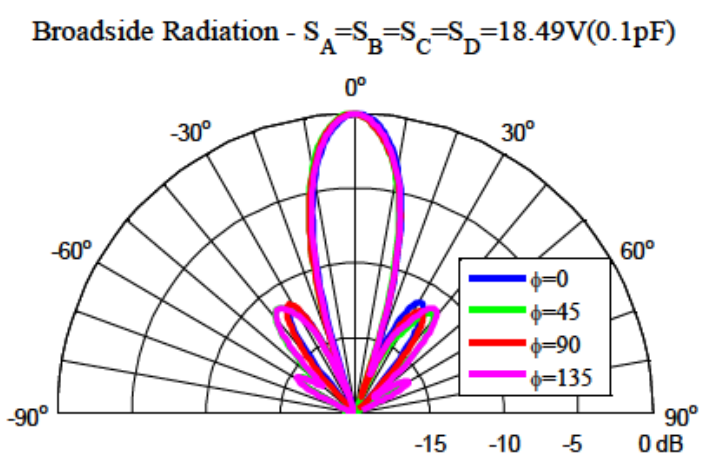

Fig. 4.4.6 Broadside configuration for optimum operating point $\left(S_{A}=S_{B}=S_{C}=S_{D}=18.49 \mathrm{~V}\right)$.

For the hybrid regime (Fig. 4.4.5(e)-(h)), the four defined configurations were tested, tuning two adjacent sectors into the scanning region (possible configurations: $\mathrm{S}_{\mathrm{A}} \& \mathrm{~S}_{\mathrm{B}}, \mathrm{S}_{\mathrm{B}} \& \mathrm{~S}_{\mathrm{C}}, \mathrm{S}_{\mathrm{C}} \& \mathrm{~S}_{\mathrm{D}}$ or $\left.\mathrm{S}_{D} \& \mathrm{~S}_{\mathrm{A}}\right)$. In this case best configuration achieved a scanning response from $\theta_{R A D}=3^{\circ}$ to $\theta_{R A D}=23^{\circ}$ for an azimuth angle of $\phi=225^{\circ}$. This configuration corresponds to $S_{C}=S_{D}$ tuned into the scanning region, and $S_{A}, S_{B}$ tuned into the EBG region $\left(S_{A}=S_{B}=2 \mathrm{~V}\right)$. A similar response from $\theta_{R A D}=3^{\circ}$ to $\theta_{R A D}=20^{\circ}$ was achieved for the rest of configurations. It is worth to note that the maximum scanning angle defined for each operating regime of the antenna has been observed when the pencil beam starts to be no longer sensitive to variations of the tuning voltage $\left(V_{R}\right)$. Beyond these maximum operating points, the maximum gain is sharply decreased due to varactors' losses as the beamwidth grows abruptly (as expected from conclusions derived in Section 2.8), reducing directivity and making the radiation patterns noisy and impractical. Finally, broadside configuration was tested, tuning all the sectors close to the splitting conditions, as was previously explained. The measured radiation pattern is shown at different cutting planes in Fig. 4.4.7 for the optimal (maximum directivity) broadside configuration, $\left(S_{A}=S_{B}=S_{C}=S_{D}=18.49 \mathrm{~V}\left(C_{j}=0.1 \mathrm{pF}\right)\right)$. A pencil beam pointing at broadside is clearly observed, with a secondary lobe level of approximately $12 \mathrm{~dB}$ for all the main planes. Measured directivity reaches $16.9 \mathrm{~dB}$, as will see below.

To sum up the scanning results obtained from the measured radiation patterns, Fig. 4.4.7 shows the measured scanning response for each TE, TM and Hybrid regime and compares with the results obtained from full-wave (FEM) simulations shown in previous section (Fig. 4.3.3). From Fig. 4.4.7(b) and Fig. 4.4.7(c) show the scanning range obtained for the TE and hybrid regimes respectively. Good agreement between simulations and measurements is appreciated. However, for the TM regime case some discrepancies have appeared, regarding the maximum scanning range achieved from simulations $\left(19^{\circ}\right)$ and from the tested prototype $\left(12^{\circ}\right)$. These discrepancies can be originated by multiple factors. However, we believe that the most important (and most likely) factor which is limiting the maximum angle for the TM leaky mode is because of dissimilarities among the electronic responses of the varactors due to their fabrication 
tolerances. As aforementioned in previous sections, the varactor diodes must present similar response in order to obtain an homogeneous electrical response from the tunable HIS sector, if not, higher mismatching and phase aberrations can produce negative effects on the scanned beam, as the abrupt increment of the beamwidth observed for the TM case in Fig. 4.4.5(a) and (b) when the antenna is pointing at higher elevation scanning angles.

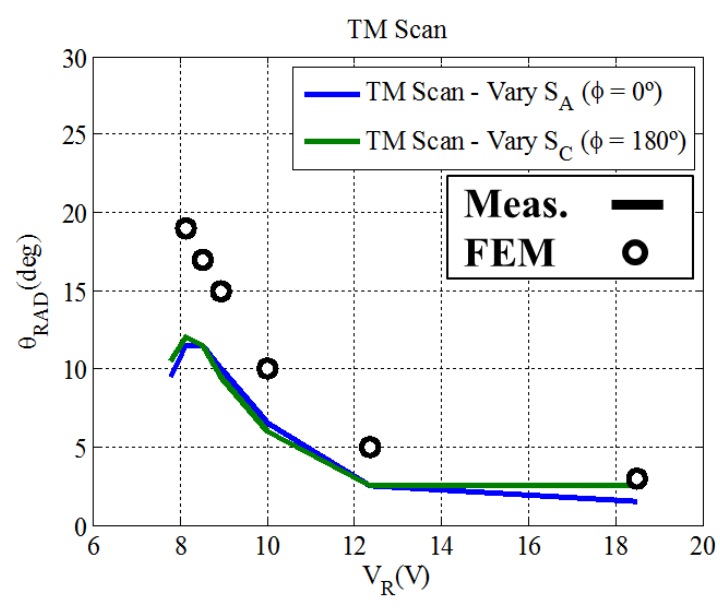

(a)

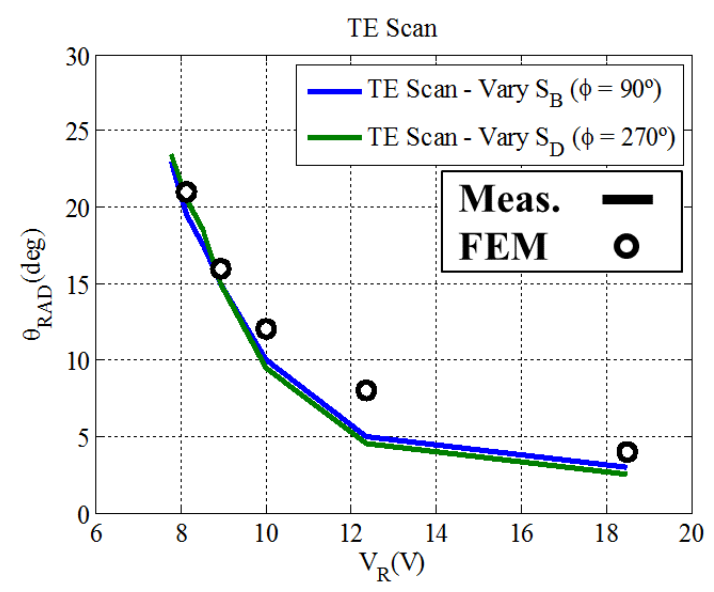

(b)

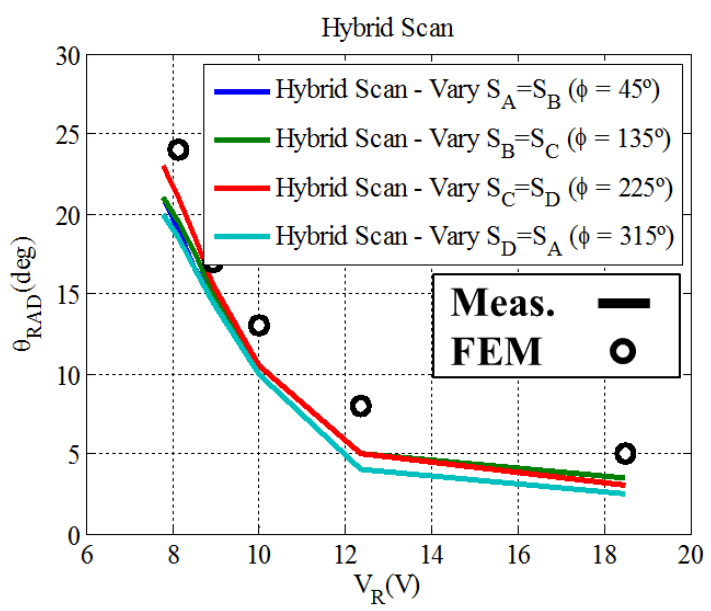

(c)

Fig. 4.4.7 Measured and simulated (FEM, ([CST 2010])) scanning angle for different configurations at (a) TM regime, (b) TE regime and (c) Hybrid regime.

Fig. 4.4.8 shows the maximum directivity and realized gain (measured and simulated) corresponding to the radiation patterns plot in Fig. 4.4.7. The $S_{11}$ is plot in Fig. 4.4.9 (also measured and simulated) for each configuration as a function of the scanning angle. For the TM regime case (Fig. 4.4.8(a)), a maximum gain of $G_{M A X}=13 \mathrm{~dB}$ is achieved with a scanning angle of $\theta_{R A D}=6^{\circ}$ for both TM configurations $\left(\phi=\left[0^{\circ}, 180^{\circ}\right]\right)$. However, a decreasing tendency of the gain is observed as the scanning angle increases, becoming to a minimum gain of $G_{M I N}=8.5 \mathrm{~dB}$ when $\theta_{R A D}=12^{\circ}$. From these results, it can be noticed that the gain falls abruptly. Simulations present a similar maximum gain of $G_{M A X}=14.3 \mathrm{~dB}$ for almost the whole range, achieving a 
minimum gain $G_{M I N}=8.5 \mathrm{~dB}$ when the maximum scanning angle is reached $\left(\theta_{R A D}=19^{\circ}\right)$. Concerning Fig. 4.4.9(a), acceptable measured mismatching $\left(S_{11}<-8 \mathrm{~dB}\right)$ is obtained for higher scanning angles $\left(\theta_{R A D}>6^{\circ}\right)$. From simulations, it can be observed a mismatching $S_{11}<-6 \mathrm{~dB}$ for $\theta_{R A D}>3^{\circ}$; which decreases in a softer way as the scanning angle increases (and bias voltage applied to the varactors in the active sectors decreases). The abrupt decreasing tendency of the measured realized gain lead us to reinforce our arguments about the premature deterioration of the gain patterns which limit the maximum scanning angle achieved by the TM leaky mode, in contrast to the predicted response obtained from simulations. Thus, the faster decrement of the gain with the scanning angle (and also the increment of the beamwidth observed in Fig. 4.4.5(a) and (b)) can be associated to some unit cells of the tunable HIS which must be operating closer to the Perfect Magnetic Condition (PMC) (due to some more sensitive varactors), as the elevation scanning angle increases, deteriorating the gain. Regarding the TE regime (Fig. 4.4.8(b)), similar measured gain has been obtained for both TE configurations $\phi=\left[90^{\circ}, 270^{\circ}\right]$. A maximum measured gain of $G_{M A X}=15.6 \mathrm{~dB}$ has been measured for a scanning angle of $\theta_{R A D}=4.5^{\circ}$, whereas minimum gain of $G_{M I N}=7.8 \mathrm{~dB}$ is obtained at the maximum scanning angle $\theta_{R A D}=23^{\circ}$. Simulated gain is quite similar for lower angles, although simulated gain is higher than measured for scanning angles above $\theta_{R A D}>10^{\circ}$, where a predicted minimum gain of $G_{M I N}=14.2 \mathrm{~dB}$ is observed when $\theta_{R A D}=21^{\circ}$. This discrepancy between simulations and measurements can be attributed again to higher losses of the varactors (not predicted by the model employed in the full-wave simulations). Acceptable simulated and measured mismatching response below $\mathrm{S} 11<-8 \mathrm{~dB}$ has been obtained for almost the whole scanning range $\left(\theta_{R A D}>7^{\circ}\right)$ of the antenna, however, simulations report a slightly better $S_{11}$ in most of the scanning range. Concerning the hybrid regime, also similar response has been obtained from the four different configurations (pointing at $\phi=\left[45^{\circ}, 135^{\circ}, 225^{\circ}, 315^{\circ}\right]$ ) and maximum gain for lower angles almost match the predicted maximum gain from simulations, achieving maximum measured gain $G_{M A X}=16 \mathrm{~dB}$ with a scanning angle of $\theta_{R A D}=4^{\circ}$, and simulated $G_{M A X}=15.04 \mathrm{~dB}$ for $\theta_{R A D}=5^{\circ}$. Again, it can be observed that the gain decreases as the scanning angle increases. In this case, the measured minimum gain has been achieved for $\theta_{R A D}=23^{\circ}$, with $G_{M I N}=4.33 \mathrm{~dB}$, whereas simulations predicted a $G_{M I N}=11.31 \mathrm{~dB}$ for $\theta_{R A D}=24^{\circ}$. Good mismatching response is observed for the whole scanning range achieved in the hybrid regime. In this case, measured $S_{11}<-6.1 \mathrm{~dB}$ for $\theta_{R A D}>4^{\circ}\left(S_{11}<-7.8 \mathrm{~dB}\right.$ for simulation results) is observed in Fig. 4.4.9(c). Best matching $S_{11}<-13.8 \mathrm{~dB}$ is observed for the highest measured scanning angles. Finally notice that the simulated directivity has been also plot in Fig. 4.4.8. From the difference between simulated directivity and simulated realized gain, it can be observed that a constant dissipative loss about $\sim 1.3 \mathrm{~dB}$ affect to the antenna performance for every operating regime and scanning angle. This means that the tunable HIS is practically presenting constant losses at any operating point because likely it is not working too close to the AMC resonance at each operating mode. 


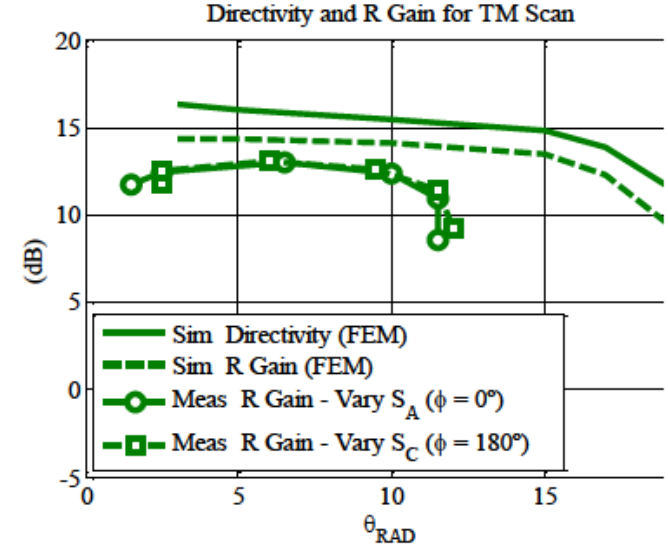

(a)

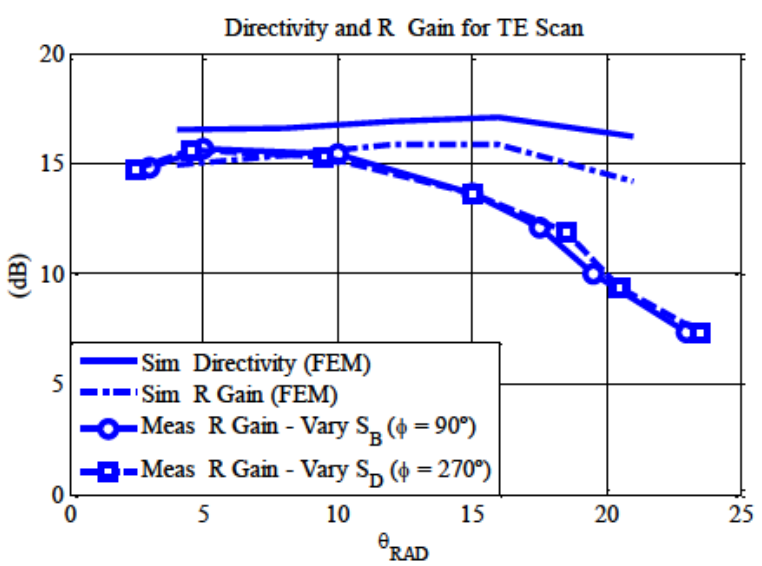

(b)

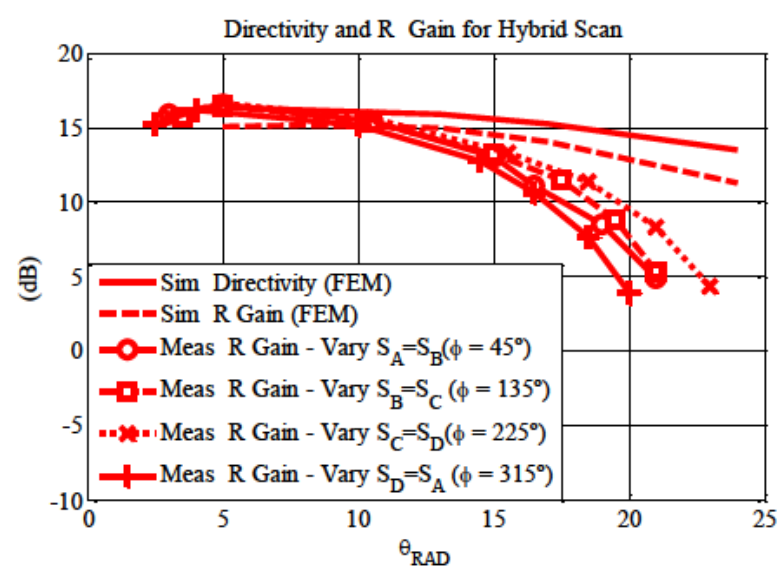

(c)

Fig. 4.4.8 Maximum Directivity (Simulated ([CST 2010])) and Realized Gain (simulated and measured) as function of the scanning angle (5.5GHz) for (a) TM scan, (b) TE scan, (c) Hybrid.

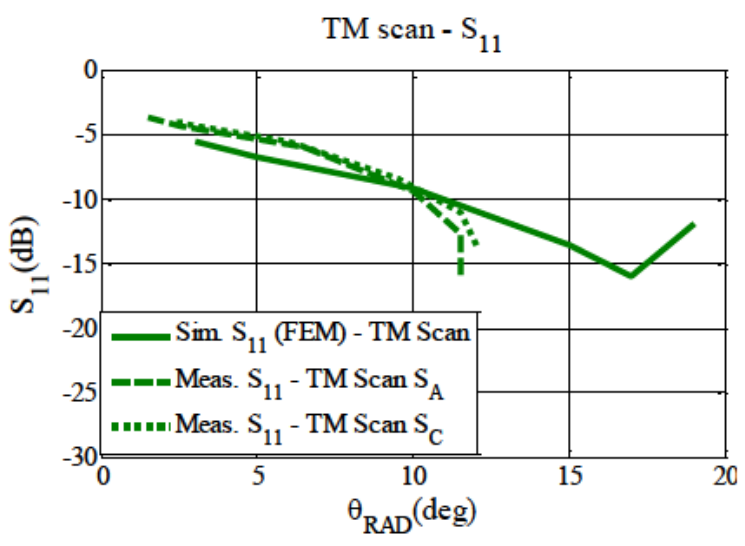

(a)

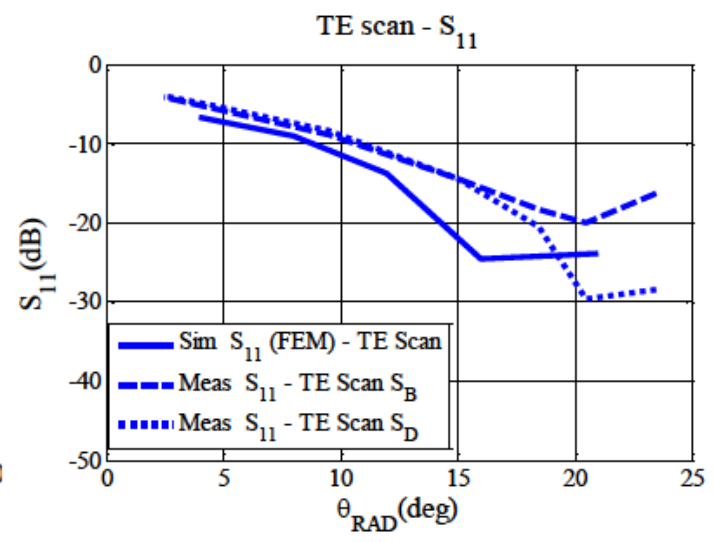

(b) 


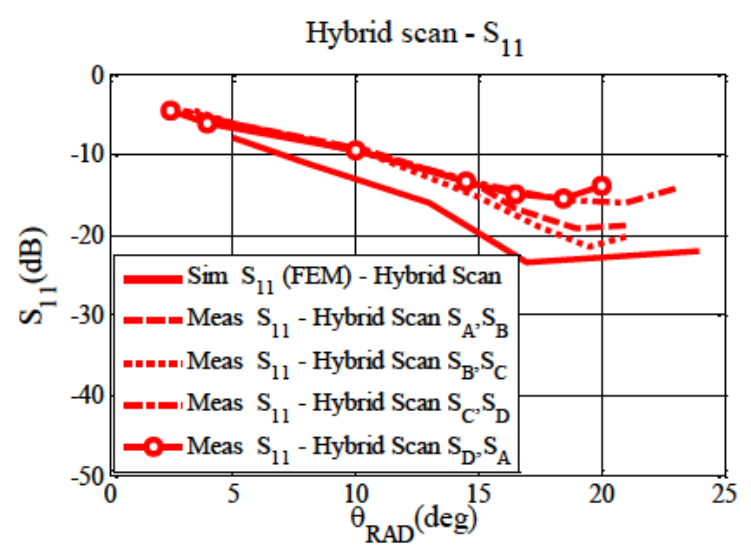

(c)

Fig. 4.4.9 Measured and simulated input matching $S_{11}$ for the Reconfigurable 2D FP LWA at

$$
\text { 5.5GHz: (a) TM scan, (b) TE scan, (c) Hybrid scan. }
$$

Finally, the broadside radiation configuration is also tested. In this case, experiments demonstrated that a maximum gain of $G_{M A X}=15.7 \mathrm{~dB}$ is obtained for the case $S_{A}=S_{B}=S_{C}=S_{D}=18.49 \mathrm{~V}\left(C_{j}=0.1 \mathrm{pF}\right)$, being this configuration the optimum operating point to achieve maximum scanning angle. Fig. 4.4.10 shows the tendency of maximum gain of the pencil beam as a function of the tuning voltage applied to the sectors of the antenna. It is clearly observed that as $V_{R}$ decreases, $G_{M A X}$ also does, falling abruptly when $S_{A}=S_{B}=S_{C}=S_{D}=12 \mathrm{~V}$, where the pencil beam becomes a conical beam ([Lovat 2006-I]). This results are already expected from simulations, which predicted a similar gain of $G_{M A X}=15 \mathrm{~dB}$ for the same operating point of the varactors $\left(C_{j}=0.1 \mathrm{pF}\right)$. A comparative table is presented below, in Table 4.4.2, where simulated and measured results are contrasted for the broadside case for the optimum point. Regarding mismatching at broadside, simulations predicted a value of $S_{11}=-8 \mathrm{~dB}$, whereas measurements presents a poorer mismatching of $S_{11}=-4.9 \mathrm{~dB}$.

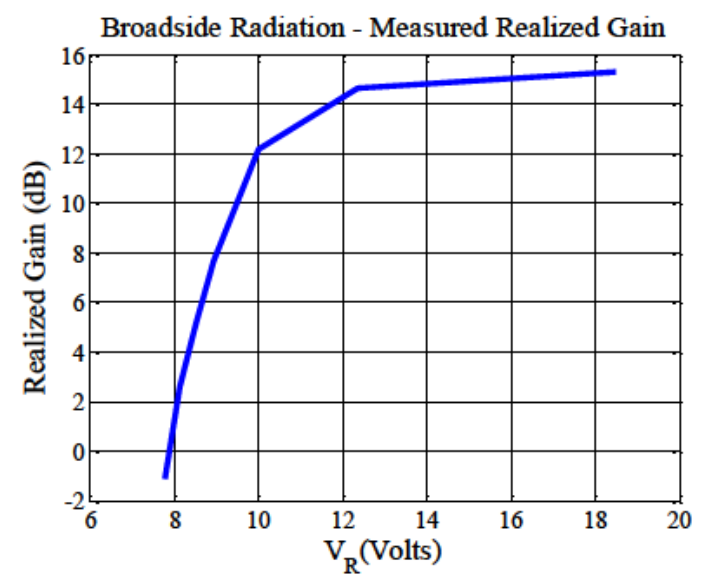

Fig. 4.4.10 Measured realized gain as a function of the operating tuning voltage $\left(V_{R}\right)$ for Broadside configuration at $5.5 \mathrm{GHz}$. 


\begin{tabular}{|c|c|c|c|c|c|c|}
\hline $\begin{array}{c}\mathbf{S}_{\mathbf{A}}=\mathbf{S}_{\mathbf{B}}=\mathbf{S}_{\mathbf{C}}=\mathbf{S}_{\mathbf{D}} \\
(\mathbf{V})\end{array}$ & $\begin{array}{c}\text { FEM } \\
\text { S11(dB) }\end{array}$ & $\begin{array}{c}\text { Meas. } \\
\text { S11(dB) }\end{array}$ & $\begin{array}{c}\text { FEM } \\
\text { Dir. (dB) }\end{array}$ & $\begin{array}{c}\text { FEM } \\
\text { R.Gain(dB) }\end{array}$ & $\begin{array}{c}\text { Meas. } \\
\text { Dir.(dB) }\end{array}$ & $\begin{array}{c}\text { Meas. } \\
\text { R.Gain(dB) }\end{array}$ \\
\hline $18.49 \mathrm{~V}$ & -8.4 & -4.9 & 16.9 & 15.0 & 16.9 & 15.7 \\
\hline
\end{tabular}

Table 4.4.2 Bias voltage, $S_{11}$, Realized Gain and Directivity (FEM and measured) for Broadside configuration at $5.5 \mathrm{GHz}$ at $18.49 \mathrm{~V}\left(C_{j}=0.1 \mathrm{pF}\right)$.

From these experimental results it has been demonstrated the proof of concept here pursued: the conception of a two-dimensional electronic scanning antenna employing a single and simple excited 2D FP cavity. The discrepancies observed between simulated and measured results for the different operating regimes of the antenna can be mostly attributed to fabrication tolerances and also to the fact that varactors likely present more losses than predicted with the RLC model used in the full-wave simulations. Again, it must be stressed that dissimilarities among the 240 varactors employed to reconfigure the structure may influence negatively the overall response, however, despite this issue, electronic scanning is clearly observed for the eight azimuthal configurations. Also note that, as mentioned for the half-space and full-space 1D FP LWAs presented in previous sections, the proper design of the excitation source is again determinant for the design of the antenna. For this 2D antenna, this design has been even more complex than for its one-dimensional counterparts. In this case, nine different operating regimes (one per each azimuth scanning angle + broadside radiation) have had to be matched from optimizing the dimensions of the central stacked patch antenna, evidencing again one of the most significant factors which complicates and challenges the practical design of these antennas: the design of the excitation source. Thus, further research on this issue will have to be analyzed in order to study other types of excitation sources and enhance the matching process design in order to speed up the design process of these antennas. However, in this work it has been demonstrated that from a simple single stacked patch antenna, acceptable mismatching response can be ensured. Regarding the TM scanning regime discrepancies, further investigation must be performed. The initial hypothesis indicate a problem with homogeneity of the tunable HIS, however, although it has not been discussed previously, it can be thought that another mode (in particular a quasi-TEM) might be also excited by the source, between the PRS and the bottom ground plane of the HIS, affecting negatively to the performance of the antenna. 


\subsection{Conclusions}

A novel reconfigurable 2D Fabry-Pérot (FP) LWA has been proposed in this chapter, with simultaneous steering of its pencil beam in azimuth and elevation, fulfilling the last objective of this thesis (O7). In general, it has been observed that normal passive 2D FP LWAs, created from a simple FP cavity with an embedded source at the center of the structure, excite cylindrical leaky waves which create high-gain pencil beams pointing at broadside, or conical beams due to its symmetric geometry. In this case, a similar 2D FP cavity is proposed, comprised by a two-dimensional patch-based PRS and a two-dimensional tunable HIS loaded with varactors. An x-polarized central excitation, constituted by an embedded probe-fed stacked patch antenna, excites polarized leaky waves which originates TM leaky modes in the $x$ direction, TE leaky modes in the $y$ direction, and hybrid TE/TM leaky modes in the rest of the radial directions. Thus, the dispersion of the TE and TM leaky modes, which propagate in the principal directions, $x$ and $y$ axis, have been analyzed, obtaining the dispersion curves as a function of the varactors' junction capacitance, at $5.5 \mathrm{GHz}$. From these curves, the scanning and EBG regions for each mode have been identified. The tunable HIS has been divided in four independently biased sectors $\left(\mathrm{S}_{\mathrm{A}}-\mathrm{S}_{\mathrm{D}}\right)$, to control the scanning or EBG region at each section of the FP cavity, and hence controlling the aperture field distribution of the 2D FP antenna. Depending on the sectors' configuration, different operating regimes can be operated: TE, TM, $\mathrm{TE} / \mathrm{TM}$ hybrid and broadside operating regimes. A fabricated prototype operating at $5.5 \mathrm{GHz}$, have been tested, confirming a continuous control of the pencil beam in elevation and discrete in azimuth, being able to point at $\left.\phi=\left[0^{\circ}, 45^{\circ}, 90^{\circ}, 135^{\circ}, 180^{\circ}, 225^{\circ}, 270^{\circ}, 315^{\circ}\right]\right)$. The elevation scanning range has been measured for each one of the eight configurations (one per azimuth direction). It has been observed that a scanning range for the TE configurations from $3^{\circ}$ to $23.5^{\circ}$ can be achieved. For the hybrid configurations, similar scanning range from $3^{\circ}$ to $20^{\circ}$ was obtained with the worst configuration, and till $23^{\circ}$ with the best. The poorer scanning obtained was with the TM configurations, being of $2.5^{\circ}$ to $12^{\circ}$ with the best configuration operating at TM regime, what disagrees with the simulation results, which predicted a scanning range for $\mathrm{TM}$ case from $3^{\circ}$ to $19^{\circ}$. These discrepancies for the TM case have been in principle associated to the employment of inhomogeneous varactors, which causes an increment of losses which particularly affects to the TM polarization, and rapidly degrades the gain of the antenna as the angle increases. Analogously to the full-space steering 1D FP LWA presented in Chapter 3, optimum broadside radiation have been also obtained with this antenna when all the sectors are properly tuned satisfying the splitting condition, obtaining in this case a pencil beam pointing at $\theta_{R A D}=0^{\circ}$. It must be noticed that only $25 \%$ of the antenna aperture is illuminated, except for the broadside radiation configuration, in that case, all the sectors are active and the aperture 
efficiency will be higher. However, this antenna could represent an interesting alternative for conceiving 2D scanning antennas because of its simplicity (it employs just 4 control signals for the steering control and the structure is composed of two easily fabricable flat surfaces and a stacked patch antenna as source) and low profile, in contrast to mechanically reconfigured reflector antennas, electronic reflectarrays or transmitarrays, which require of external sources. Also, it is thought that this antenna presents potential for scalability; the technology in which this antenna is implemented can be easily scaled to millimeter or even $\mathrm{THz}$ frequencies with the current manufacturing technologies, and in addition, the structure allows the integration of other tuning devices more convenient for such higher frequencies (MEMS, liquid crystal, piezoelectric actuators...). This advantages are not found in other technologies such as phased arrays ([Hansen 1998], [Rock 2008], [Manasson 2010], [Raisanen 2012]), because of the type of structure and reconfiguration mechanism on which this antennas are based. The simplicity of this structure, in terms of integrated excitation and its low profile (just a few centimeters at $5.5 \mathrm{GHz}$ ), could open up interesting alternatives for practical reconfigurable antennas with electronic steering capabilities.

The work developed in this chapter has given rise to the publication of 2 peer-review international journal paper, 1 Spanish journal papers, 1 international conference papers and 1 Spanish conference papers. These references are copied below; they have been extracted from the complete list of publications of Section 5.3.

IJ3. Y.J. Guo, J.L. Gomez-Tornero, R. Guzmán-Quirós, and A.R. Weily, "Reconfigurable Leaky-Wave Antennas," Forum for Electromagnetic Research Methods and Application Technologies (FERMAT), vol.1, no., pp., January 2014, available online : http://www.efermat.org/files/articles/1533721af95bd9.pdf

IJ5. R. Guzmán-Quirós, J.L. Gomez-Tornero, A.R. Weily, and Y.J. Guo, "Novel Fabry-Pérot LWA with Two-Dimensional Electronic Beam-Scanning Capability," IEEE Trans. Antennas Propag., vol., no., pp., submitted.

IC5. R. Guzmán-Quirós, J. L. Gómez Tornero, M. García-Vigueras, A.R. Weily, Y. J. Guo, "Advances in electronically reconfigurable LWAs in Fabry-Pérot and SIW technologies", 7th European Conference on Antennas and Propagation (EUCAP 2013), pp. 224-228, April 2013. (Invited contribution to a convened session about "Emerging Antenna Technologies" at EuCAP 2013)

SJ6. R. Guzmán Quirós, A. J. Martínez Ros, J. L. Gómez Tornero, "Escaneo electrónico del haz de radiación en Antenas Leaky-Wave 2D empleando superficies de alta impedancia 
activas", VI Jornadas de Introducción a la Investigación de la UPCT (ISBN: 1888-8356), vol.6, pp.127-129, 2013.

SC9. R. Guzmán Quirós, J. L. Gómez Tornero, A. J. Martínez Ros, Andrew R. Weily, Y. J. Guo, "Novel and Simple Electronically Reconfigurable Fabry-Pérot Antennas", XXVIII URSI (International Scientific Radiocommunications Union) Symposium, Santiago de Compostela, Spain, 11-13 Septiembre 2013. (First Prize Young Scientists URSI 2013) 


\section{Chapter 5 Conclusions and Future Lines}

In this chapter it is summarized the main conclusions achieved during the development of this thesis. Several ideas for improving the proposed structures and future research lines to propose novel structures derived from the results of this thesis are also described.

\subsection{Conclusions}

As it was explained in the first chapter of this thesis, a wide variety of reconfigurable antennas have been studied, especially in the recent decades, given the increasing interest for additional functionality for current and future communication and RF-sensing systems, or the new paradigms which are arising with the exponential expansion of wireless communications. In this context, several fields are involved in the advances of reconfigurable antennas, such as microelectronics or material engineering, which are looking for new types of switching and smart materials to conceive new simple and low cost reconfiguration mechanisms, also intended for reconfigurability at higher frequency bands. Then, the current tendency is to contribute with new structures with higher compactness and lower cost, in order to provide affordable systems in the future. In this way, LWAs seem to fit well with these demands. In fact, these antennas are currently one of the most investigated topics to achieve new reconfigurable antennas. In the framework of this thesis, we have focused on the potentials offered by Fabry-Pérot (FP) LWAs. These structures are mainly characterized by its simple and low profile structure, with an embedded source to excite the antenna, providing high gain characteristics. These 
characteristics make them interesting to conceive novel reconfigurable antennas for long-range communication systems required in base stations, satellites or airborne platforms, where space might be reduced and shared with other systems, or where streamlined architectures could be of major interest.

The main contribution of this thesis has been the conception of novel Fabry-Pérot LWAs (FP LWAs) with one-dimensional (1D) and two-dimensional (2D) electronic beam-steering capabilities, employing a reduced number of control signals, simple dispersion-based reconfiguration mechanisms and low cost technology. The development of a simple, but powerful analysis tool to analyze the proposed structures has been crucial for the development of this thesis. This efficient tool, based on the Transverse Resonance Method (TRM), have allowed to predict, in a fast and accurate way, the response of the reconfigurable antennas, to analyze its dispersion and ultimately optimize the dimensions of the structure. Therefore, it can be said that this tool has served as a quick design guideline to obtain the initial dimensions of these antennas. Otherwise, unfeasible optimization processes by full-wave simulations would have had to be performed, what had complicated much more the investigation process and the design of such antennas. In addition, the complexity of the proposed structures have been gradually increased with each chapter, starting from a simpler 1D FP LWA for half-space beamsteering, passing through a full-space beam-steering 1D FP LWA, and ending up with a 2D FP LWA with electronic-steering in elevation and azimuth. This ascension in functionality, which has been also chronological during the development of the investigation, would not have been possible without the achievement of each one of the objectives which have been pursued throughout this thesis step by step. Below, these achievements are summarized by each chapter.

In Chapter 2, it has been demonstrated how a 1D FP LWA can attain beam-steering at fixed frequency by employing a Fabry-Pérot (FP) cavity comprised by a top Partially Reflective Surface (PRS) and a tunable High Impedance Surface (HIS) with varactor diodes located at the bottom of the cavity. This antenna is excited from one end by a $\mathrm{TE}_{01}$ mode, generating a fan beam narrow in the H-plane (the scanning plane), and wide in the transversal E-plane. Here, it is described the structure, and the Transverse Equivalent Network (TEN) which has been employed to apply the TRM and find the propagation constant of the leaky modes excited in the structure. Also, in this chapter have been described the pole zero methods employed to characterize as equivalent admittances the frequency selective surfaces (FSSs) employed to constitute the PRS, and the tunable FSS of the HIS. A dispersion analysis in frequency and with respect to the variable capacitance of the varactor diodes $(C j)$ at fixed frequency $(5.6 \mathrm{GHz})$, evidenced theoretically the steerability of the pointing angle in the first quadrant (positive angles), as a function of the varactors' capacitance, which was the initial hypothesis of this work. This concept has been demonstrated by simulations and confirmed by experimental 
results of a fabricated prototype designed to operate at $5.6 \mathrm{GHz}$ and with an aperture length of $L_{A}=5.2 \lambda_{0}$ at $5.6 \mathrm{GHz}$. A coaxial-to-waveguide transition was designed to excite the antenna from one end. From the tests, a scanning range from $9^{\circ}$ to $30^{\circ}$ has been observed, at a fixed frequency of $5.6 \mathrm{GHz}$, as the varactors' bias voltage is varied from $18 \mathrm{~V}$ to $5 \mathrm{~V}$. Notice, that the scanning range is limited by the decreasing gain, caused by the dissipative losses of the varactors and dielectric materials, and the mismatching of the source. In order to analyze in detail the antenna radiation efficiency, estimations of the losses due to the varactors and the dielectric substrates were performed. The antenna presents radiation efficiency close to $70 \%$ for most part of the scanning range, which is reduced as the pointing angle scans to endfire. This reduction of efficiency is also noticed in the gain, which is reduced from $12 \mathrm{~dB}$ to $5 \mathrm{~dB}$ at $30^{\circ}$. Thus, it is concluded that the operating point of the tunable HIS is determined by the efficiency of the antenna, presenting maximum losses due to the varactors and dielectric materials when the HIS is close to operate as a Perfect Magnetic Conductor (PMC). Finally, the study of the pattern bandwidth for reconfigurable beam-scanning LWAs has been tackled, developing a formula to estimate the pattern bandwidth from the information obtained from the frequency dispersion curves of the LWA. In particular, estimations performed for the half-space electronic-steering 1D FP LWA predicted a pattern bandwidth of $2.26 \%$, whereas bandwidth estimated from lossy full-wave simulations estimated a $2.22 \%$ at $5.6 \mathrm{GHz}$, what agrees quite well, despite the approximations assumed in the formulas. Also, it has been evidenced that pattern bandwidth is mainly affected by the beamwidth (and hence the length of the LWA), the pointing angle and also the decreasing of the gain due to the effect of varactors' losses. Therefore, objectives O1, O2, $\mathbf{O 3}$ and 04 enlisted in Section 1.2 have been achieved.

Chapter 3 presents a novel full-space beam-steering 1D FP LWA, able to scan at negative, positive angles and also able to point at broadside (note that broadside was not attained from the previous half-space LWA excited from one end). This approach is simple, in terms of structure complexity, since do not need of metamaterials, periodic structures or additional SPDT switches to achieve backward-to-forward scanning. Instead, the previous half-space 1D FP LWA was modified, being excited from its center, and divided in two half-space LWAs with a single common source, and each one loaded with an independently biased tunable HIS. In addition, in this chapter it is demonstrated that exists an EBG region appeared because of the introduction of the periodic HIS, which prevents the propagation of any leaky mode inside the cavity. Thereby, this EBG region has been employed to act as an electronic-routing mechanism which allows to reroute the leaky waves launched from the central excitation to the desired halfspace LWA. Thus, it is possible to scan at the first quadrant (positive angles), when the right LWA is propagating, and the left one is at EBG, or at second quadrant (negative angles) in inverse configuration. Also, optimal broadside radiation is achieved when both sides of the 
antenna are tuned at the splitting condition, where a single narrow beam is achieved pointing at that direction. A fabricated prototype confirmed this reconfiguration concept, observing a scanning range from $\mathbf{- 2 5}^{\mathbf{0}}$ to $\mathbf{+ 2 5}^{\mathbf{0}}$. The central coaxial, and two additional screws where employed to match the antenna for every operating regime. The main drawback of this antenna is that when scanning at forward or backward quadrants, half of the aperture is employed, so just the aperture efficiency is reduced by a 50\%, however, this electronic-routing mechanism employed allows to extend this antenna to a 2D version for two-dimensional scanning, as demonstrated in next chapter. With these results, objectives $\mathbf{0 5}$ and $\mathbf{O 6}$ are accomplished.

Chapter 4 concludes with the analysis and design of a 2D FP LWA for two-dimensional electronic beam-steering capability at fixed frequency. Unlike previous 1D LWAs, which scanned fan beams, this 2D structure produces a pencil beam narrow in both E- and H-planes, which is able to be steered continuously in elevation, and at eight different azimuth angles. The antenna consists of a two-dimensional tunable HIS divided in four independently biased sections $\left(\mathbf{S}_{\mathbf{A}}-\mathbf{S}_{\mathbf{D}}\right)$. An $\mathbf{x}$-polarized central excitation, constituted by an embedded probe-fed stacked patch antenna, excites a polarized cylindrical leaky wave which originates TM leaky modes in the $x$ direction, TE leaky modes in the $y$ direction, and hybrid TE/TM leaky modes in the rest of the radial directions. Thus, the dispersion of the TE and TM leaky modes, which propagate in the principal directions, $x$ and $y$ axis, have been analyzed, obtaining the dispersion curves as a function of the varactors' junction capacitance, at $5.5 \mathrm{GHz}$. From these curves, the scanning and EBG regions for each mode have been identified. The tunable HIS has been divided in four independently biased sectors $\left(\mathrm{S}_{\mathrm{A}}-\mathrm{S}_{\mathrm{D}}\right)$, to control the scanning or EBG region at each section of the FP cavity, and thereby controlling the aperture field distribution of the 2D FP antenna. Depending on the sectors' configuration, different operating regimes can be operated: TE, TM, TE/TM hybrid and broadside operating regimes. A fabricated prototype operating at $5.5 \mathrm{GHz}$, have been tested, confirming a continuous control of the pencil beam in elevation at discrete azimuth angles, being able to point at $\phi=\left[0^{\circ}, 4^{\circ}, \mathbf{9 0}^{\circ}, \mathbf{1 3 5}^{\circ}, \mathbf{1 8 0}^{\circ}\right.$, $\left.\mathbf{2 2 5}^{\circ}, \mathbf{2 7 0}^{\circ}, \mathbf{3 1 5}^{\circ}\right]$ ). The elevation scanning range has been measured for each one of the eight configurations (one per azimuth direction). It has been observed that a scanning range for the TE configurations from $3^{\circ}$ to $23.5^{\circ}$ can be achieved. For the TE/TM hybrid configurations, similar scanning range from $3^{\circ}$ to $\mathbf{2 0}^{\circ}$ was obtained for the worst configuration, and till $23^{\circ}$ in the best. The poorer scanning range was obtained for the TM configurations, being from $\mathbf{2 . 5}^{\circ}$ to $12^{\circ}$ for the best configuration, what disagrees with the simulation results, which predicted a scanning range for $\mathrm{TM}$ regime from $3^{\circ}$ to $19^{\circ}$. These discrepancies for the TM case have been in principle attributed to the employment of inhomogeneous varactors, which causes an increment of losses which particularly affects to the TM polarization, and rapidly degrades the gain of the antenna as the angle increases. Analogously to the full-space electronic-steering 1D 
FP LWA presented in Chapter 3, optimum broadside radiation have been also obtained with this antenna when all the sectors are properly tuned satisfying the splitting condition, obtaining in this case a pencil beam pointing at $\theta_{R A D}=0^{\circ}$. It must be noticed that this approach only employs approximately the $25 \%$ of the antenna aperture, except for the broadside radiation case (which employs all the sectors in the scanning region). However, this antenna could represent an interesting alternative for conceiving 2D scanning antennas because of its simplicity, compactness and low profile, in contrast to other solutions such as arrays of electronically reconfigured LWAs or electronically reconfigurable reflector antennas, which require of external sources. Thus, last objective $\mathbf{O} 7$ is attained with the results obtained in this chapter. 


\subsection{Future Lines}

Throughout the design process of the prototypes presented in this thesis, several factors have been observed that reduce the efficiency, bandwidth and scanning range of the antenna, and hence their practical applicability. New lines for the study and suppression of these negative effects have been derived from these observations. One of the most important factors which has limited the scanning range is dissipative losses due to the tunable HIS employed at the bottom of the FP cavities. Losses are inherent to any structure, so it is not possible to mitigate at all, but merely reduce them. In this case, most easy and trivial solutions go through employing dielectric and metals with lower losses, or employing different materials. Also, a different alternative consists of employing another type of tuning elements with reduced losses, such as MEMS ([Chicherin 2006, 2011-I, II], [Christodoulou 2012], [Haider 2013], [Yashchyshyn 2010], [Zvolensky 2010], [Debogovic 2014-I]), instead of varactor diodes. Another option, but more difficult, because probably would require a redesign of the geometry of the structures, would be employing less resonant surfaces than tunable HIS, or design the FP cavity to operate far from the AMC resonance of the HIS. Remember that losses were a determinant when this tunable HIS was operating close to the PMC region, as was concluded in Chapter 2. The reason is related to the high currents which are excited in the metallic patches due to the AMC resonance which arise when the HIS is reflecting the wave with a reflection phased of $0^{\circ}$ ([Costa 2010]). This current is directed through the varactors, which have associated an ohmic resistance (as observed from the equivalent circuit represented in Fig. 2.5.8(b)) which increase the inefficiency of the antenna. Also, another important factor observed was mismatching. Given the high number of operating modes of the reconfigurable antennas proposed, especially for the last 2D FP LWA presented in Chapter 4 (for which up to 9 different operating regimes were defined), it is difficult to obtain from a single optimized non-reconfigurable source, acceptable matching levels in the whole dynamic range. In this sense, mismatching could be reduced employing some type of reconfigurable input networks, with the capability of match the antennas as the operating mode is changed. This type of "reconfigurable source" was employed for example in [Debogovic 2010], where an input network constituted by a varactor-loaded stub was employed to improve matching readapting its reactance for each operating state of the reconfigurable LWA. However, these solutions require of a more complex designs of the feeding networks, and in most cases, additional control signals which tune the circuits when the antenna is reconfigured.

Concerning future applications, it is thought that the antennas presented in this thesis might have potential for scalability to operate at higher frequency bands. This potential resides in their simplicity, and in the reconfigurable mechanisms on which they are based. The 
technologies employed here can be perfectly miniaturized and integrated with other tuning devices more convenient for millimeter-waves or $\mathrm{THz}$ frequencies (MEMS, piezoelectric actuators, liquid crystal...). This advantage is not always found in every technology. As an example, it was mentioned in the introduction of this thesis that phased arrays ([Hansen 1998], [Rock 2008], [Manasson 2010], [Raisanen 2012]) present several technical issues when the operating frequency is increased. In this field, recent innovations have been published from EPFL, where it has been developed for first time an original fixed-frequency beam-steering LWA in graphene to operate at $\mathrm{THz}$ bands ([Esquius-Morote]). Many references regarding reconfigurable LWAs and other technologies for mm-wave applications can be found in [Raisanen 2012].

The results presented in this thesis can be considered as a first step for more innovative future structures in the field of reconfigurable antennas. The author is currently investigating new ideas which might suppose a step forward towards more compact and multi-functional antennas. In this sense, two principal lines are being currently investigated.

First line aims to improve the 2D FP LWA steering capability exploring the possibility of achieving continuous and simultaneous steering in azimuth and in elevation. Remember that the 2D FP LWA proposed in Chapter 4, was able to scan in elevation continuously at eight different azimuth angles. This comes from the different operating regimes which were defined from tuning the four discrete sectors which divided the FP structure. Oblique azimuth angles were obtained when the antenna was reconfigured with two adjacent sectors operating at the same point inside the scanning region, while the rest are remained at EBG (prevented propagation of leaky waves through such regions of the FP cavity). Therefore, the elevation angle was chosen as a function of the scanning operating point in which the "active" sectors are working, whereas the azimuth angle is a function of the chosen sectors' reconfigured to allow propagation. The hypothesis for obtaining continuous azimuth scanning is based on varying independently the scanning point at which is operating each one of the adjacent sectors activated in the scanning region (propagating). Thus, a control of the resultant radial direction of the wave is achieved, as illustrated in Fig. 5.2.1(a), where three examples with different scanning operating points are tuned in sectors $S_{B}$ and $S_{C}\left(S_{A}\right.$ and $S_{D}$ tuned at $\left.E B G\right)$. Full-wave simulations have predicted how the leaky wave changes its propagation direction, so a pencil beam arises scanned continuously in azimuth, as can be observed in Fig. 5.2.1(b), where the associated aperture fields and radiation patterns are depicted. At this point, further tests are being developed with multiple configurations of two adjacent sectors in order to find out the maximum elevational and azimuthal scanning ranges possible with the 2D FP LWA prototype presented in Chapter 4. 

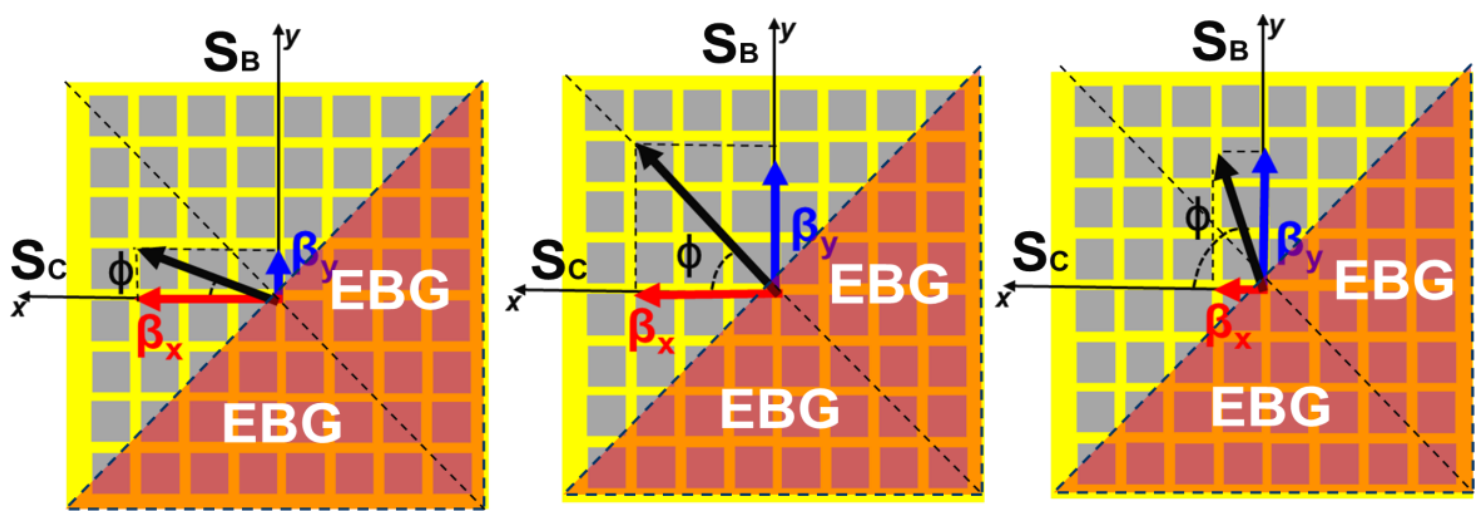

(a)
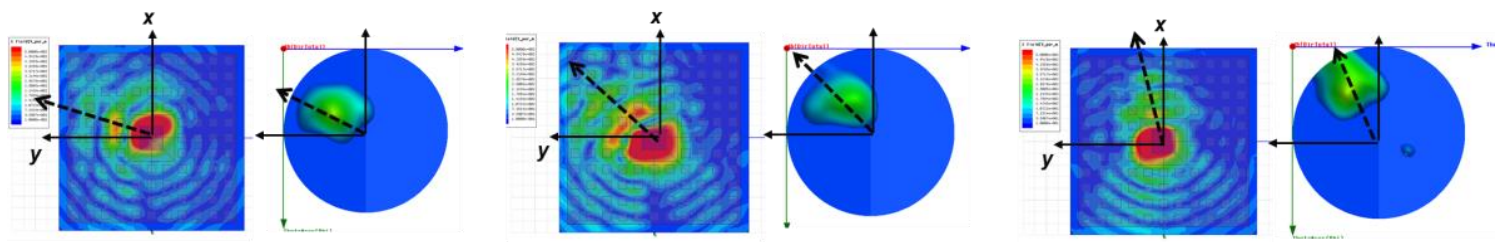

(b)

Fig. 5.2.1 (a) Top view of the beam-steering 2D FP LWA. $S_{A}$ and $S_{B}$ are independently tuned at different points in the scanning region to control the wave vector of the hybrid (TE/TM) leaky mode in azimuth and elevation. (b) Associated aperture field for three configurations of the adjacent sectors ([CST 2010]).

Second line which is also being developed consists of a pattern reconfigurable FP LWA with simultaneous control of the pointing angle $\left(\theta_{R A D}\right)$ and the beamwidth $(\Delta \theta)$ of the radiation pattern. Not so much works have proposed reconfigurable LWAs with such type of pattern reconfiguration capabilities. For example, in [Lim 2004-II] was proposed for first time a CRLH line with electronic beam-steering and also beam-shaping capabilities by modulating the phase constant along the line. Recently, in [Devogobic 2014-II] was proposed a FP LWA with beamwidth and beam-steering control. One of the key of our approach is that it is fully integrated in planar Substrate Integrated Waveguide (SIW) technology. This technology has been quite studied in the last decade because of its planarity, low loss and easiness of integration with microwave circuits ([Deslandes 2001, 2002], [Xu 2005]), what has a high practical interest to develop novel planar and low cost LWAs ([Alphones 2013], [Deslandes 2005], [Gomez 2013-I], [Hong 2007], [Martínez-Ros 2012-I, II, 2013-I, II], [Machac 2013], [Suntives 2011, 2012]). 


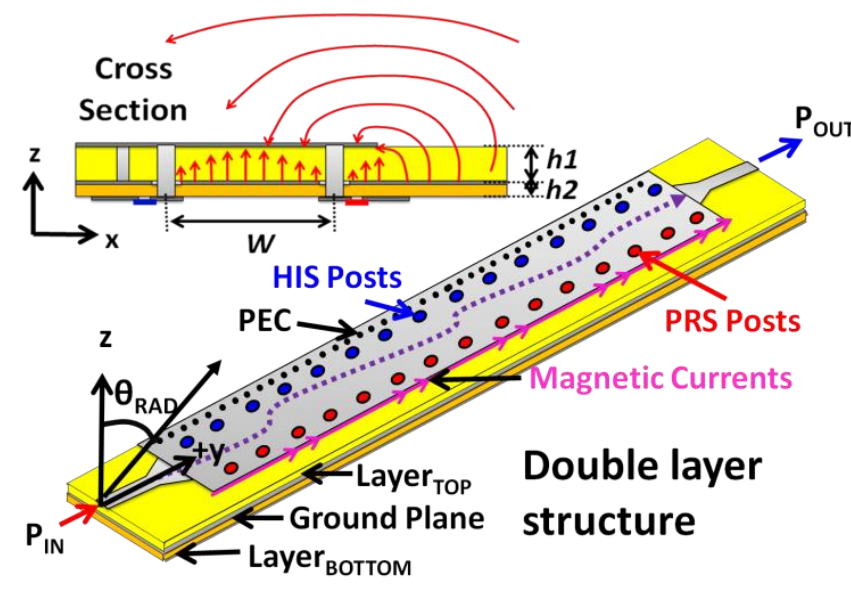

(a)

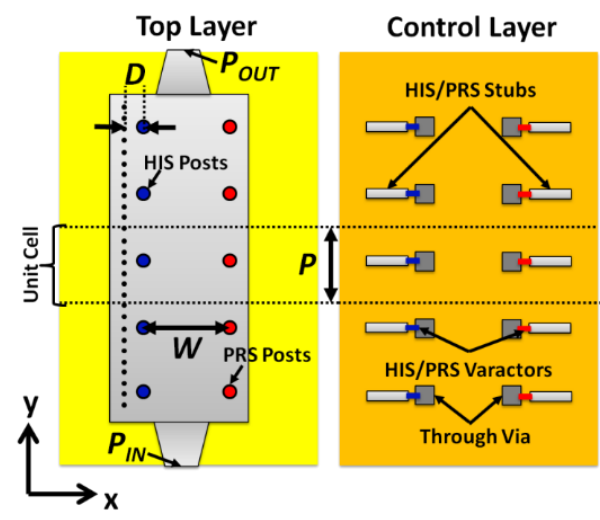

(b)

Fig. 5.2.2 Schematic of proposed reconfigurable 1D FP LWA in SIW technology. (a) 3D model and cross section. (b) Front and rear views of the structure and defined unit cell (Teflon substrates with $\varepsilon_{l}=2.2$ and thicknesses $h_{l}=3.17 \mathrm{~mm}$ and $h_{2}=0.127 \mathrm{~mm}$ are used for the top and bottom layers, respectively).

In our approach, the control of the pointing angle and of the beamwidth is integrated by a tunable HIS, which mainly controls the phase constant $(\beta)$ of the leaky wave, and a tunable PRS, which principally tune the leakage rate $(\alpha)$ of the leaky mode. The tunable HIS and PRS comprise the FP cavity which constitutes the FP LWA in SIW. The structure is inspired in the topology of a recently proposed passive LWA in SIW, which has demonstrated flexible control mechanism of the leaky wave propagation constant ([Gomez 2013-I], [Martínez-Ros 2012-I, II, 2013-I, II]). A simplified schematic of the initial structure proposed to implement this reconfigurable antenna is depicted in Fig. 5.2.2. The structure is based on a double-layer topology composed by a top layer, where the leaky-wave guide itself is integrated, and a bottom layer where varactors and control bias networks are placed. Both layers are separated by a ground plane. In the top layer, it can be observed that the HIS and the PRS, are mainly constituted by periodic arranges of metallic posts, connected to the bottom layer through via holes. The PRS and the HIS posts are separated in the $x$-direction a distance $W$ (the equivalent to the height $H$ of the FP LWAs proposed in this thesis). Hence, $W$ controls the resonance frequency of this particular planar FP LWA. The posts of the HIS has the particularity to be close to another denser array of metallic posts which acts as a ground plane, in order to grant the AMC resonance which characterize any HIS ([Sievenpiper 1999]). Two control signals allow a separated control of the PRS and the HIS. 


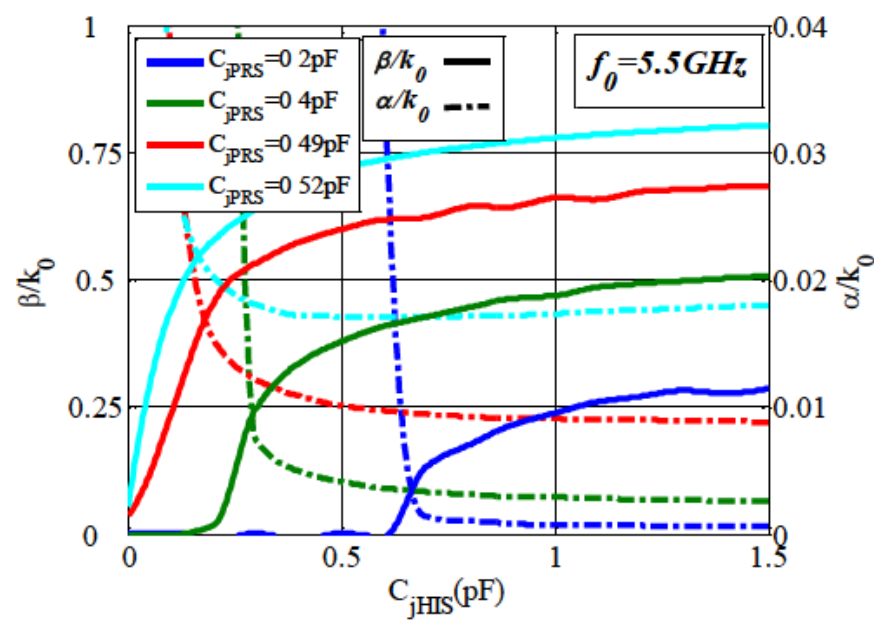

Fig. 5.2.3 Normalized dispersion curves $\left(\beta / k_{0}\right.$ and $\left.\alpha / k_{0}\right)$ as a function of $C_{j H I S}$ for different $C_{j P R S}$ values, operating at a fixed frequency of $5.5 \mathrm{GHz}$.

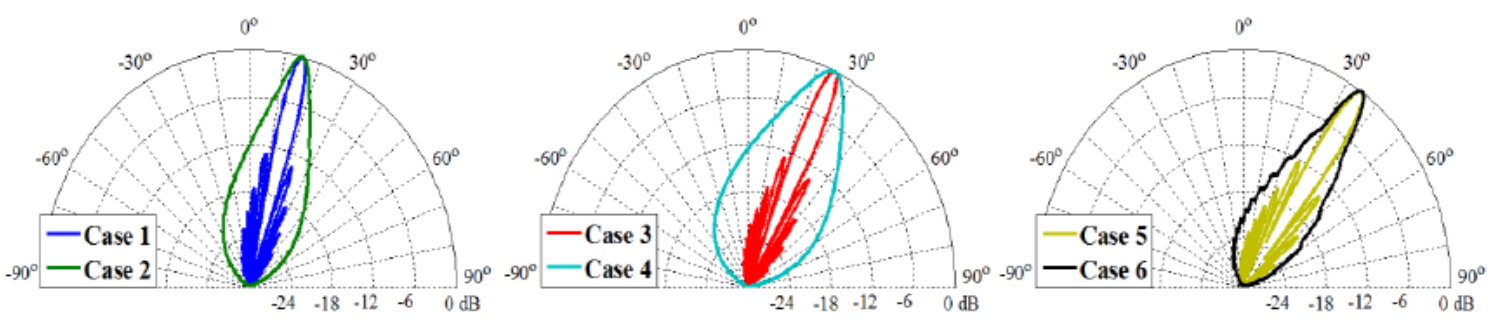

Fig. 5.2.4 Theoretical radiation patterns (normalized directivity in $\mathrm{dB}$ ) obtained for different $C_{j H I S} \& C_{j P R S}\left(\beta / k_{0} \& \alpha / k_{0}\right)$ configurations operating at $5.5 \mathrm{GHz}$.

\begin{tabular}{ccccccc}
\hline Case \# & $\begin{array}{c}\boldsymbol{C}_{j H I S} \\
(\mathbf{p F})\end{array}$ & $\begin{array}{c}\boldsymbol{C}_{j P R S} \\
(\mathbf{p F})\end{array}$ & $\boldsymbol{\beta} / \boldsymbol{k}_{0}$ & $\begin{array}{c}\boldsymbol{\theta}_{\boldsymbol{R} A D} \\
(\mathbf{d e g})\end{array}$ & $\boldsymbol{\alpha} / \boldsymbol{k}_{0}$ & $\begin{array}{c}\boldsymbol{\Delta} \boldsymbol{\theta} \\
(-\mathbf{1 0 d B})\end{array}$ \\
\hline $\mathbf{1}$ & 1,09 & 0,20 & 0,2588 & $15^{\circ}$ & 0,0007 & $7,28^{\circ}$ \\
$\mathbf{2}$ & 0,08 & 0,50 & 0,2588 & $15^{\circ}$ & 0,0630 & $22,25^{\circ}$ \\
$\mathbf{3}$ & 2,23 & 0,30 & 0,4226 & $25^{\circ}$ & 0,0009 & $7,74^{\circ}$ \\
$\mathbf{4}$ & 0,07 & 0,53 & 0,4226 & $25^{\circ}$ & 0,0930 & $33.96^{\circ}$ \\
$\mathbf{5}$ & 1,14 & 0,45 & 0,5736 & $35^{\circ}$ & 0,0050 & $8,64^{\circ}$ \\
$\mathbf{6}$ & 0,15 & 0,55 & 0,5736 & $35^{\circ}$ & 0,0390 & $17,68^{\circ}$ \\
\hline
\end{tabular}

Table 5.2.1. Radiation angle $\theta_{R A D}$ and $-10 \mathrm{~dB}$ beamwidth $\left(\Delta \theta_{-10 d B}\right)$ values obtained for each $\left(C_{j H I S}, C_{j P R S}\right)$ configuration (Antenna aperture length considered: $\left.L_{A}=12 \lambda_{0}\right)$.

In this case, the radiation mechanism is based on varying the bias voltages which control the HIS and the PRS resonances. The control of the pointing angle is based on the same principles based on the tunable HIS, explained for the half-space beam-steering 1D FP LWA presented in Chapter 2. However, the leakage rate which controls the beamwidth of the antenna, will be controlled through the tunable transparency of the PRS. Therefore, simultaneous and independent control of both parameters is achieved at fixed frequency. This hypothesis has been 
evidenced from preliminary dispersion curves obtained from full-wave simulations of the unit cell of the structure (Fig. 5.2.2(b)). Fig. 5.2.3 shows the dispersion curves as a function of $C_{j H I S}$, for different values of $C_{j P R S}$, at fixed frequency of $5.5 \mathrm{GHz}$. Below, in Fig. 5.2.2, theoretical radiation patterns have been obtained for different combinations of $C_{j H I S}$ and $C_{j P R S}$ extracted from the dispersion curves. Table 5.2.1 shows the numerical values taken, and the pointing angle and beamwidth obtained for each selected case. The reader is referenced to publications IJ3, IC5, IC6, IC7 and SJ8, enlisted in Section 5.3, where preliminary results have been published. 


\subsection{List of Publications}

During the research stage, the author of this Ph.D. has authored or co-authored several technical works derived from the results achieved in this thesis or related with the topic of leaky-wave antennas. Specifically, the author has contributed to the scientific community with 3 peerreview international journal papers, 7 international conferences, 10 national journal papers and 10 national conferences. At present, 2 additional papers for peer-review international journals are in submission process (IJ4, IJ5). Next, the references to the published contributions are listed. 


\subsubsection{International Journals}

IJ1. R. Guzman-Quirós, J. L. Gomez-Tornero, A. R. Weily, and Y. J. Guo, "Electronically steerable 1-D Fabry-Perot leaky-wave antenna employing a tunable high impedance surface," IEEE Trans. Antennas Propag., vol. 60, no. 11, pp. 5046 -5055, Nov. 2012.

IJ2. R. Guzmán-Quirós, J.L. Gomez-Tornero, A.R. Weily, and Y.J. Guo, "Electronic full-space scanning with 1-D Fabry-Pérot LWA using electromagnetic band-gap," IEEE Antennas and Wireless Propagat. Lett., vol.11, no., pp.1426-1429, 2012.

IJ3. Y.J. Guo, J.L. Gomez-Tornero, R. Guzmán-Quirós, and A.R. Weily, "Reconfigurable Leaky-Wave Antennas," Forum for Electromagnetic Research Methods and Application Technologies (FERMAT), vol.1, no., pp., January 2014, available online : http://www.efermat.org/files/articles/1533721af95bd9.pdf

IJ4. R. Guzman-Quirós, J. L. Gomez-Tornero, A. R. Weily, and Y. J. Guo, "Dynamic Scanning Pattern Bandwidth in Electronically-Steerable Leaky-Wave Antennas," IEEE Antennas and Wireless Propagat. Lett., vol., no., pp., submitted.

IJ5. R. Guzmán-Quirós, J.L. Gomez-Tornero, A.R. Weily, and Y.J. Guo, "Novel Fabry-Pérot LWA with Fixed-Frequency Two-Dimensional Electronic Beam-Steering Capability," IEEE Trans. Antennas Propag., vol., no., pp., submitted. 


\subsubsection{International Conference Proceedings}

IC1. M. Garcia-Vigueras, J. L. Gómez-Tornero, R. Guzmán-Quirós, Quesada-Pereira, F., A. Alvarez-Melcon, "Control of the radiation properties of a FSS loaded leaky-wave antenna", 4th European Conference on Antennas and Propagation (EUCAP 2010), Barcelona, Spain, April 2010.

IC2. M. Garcia-Vigueras, R. Guzmán-Quirós, J. L. Gómez-Tornero, "Beamwidth control of 1D LWA radiating at broadside", 5th European Conference on Antennas and Propagation (EUCAP 2011), Rome, Italy, April 2011.

IC3. R. Guzmán-Quirós, J. L. Gómez Tornero, M. García-Vigueras, A. R. Weily, Y. J. Guo, "Novel Topology of Fabry-Perot Electronically Steerable Leaky-Wave Antenna", 6th European Conference on Antennas and Propagation (EUCAP 2012), pp. 224-228, March 2012. (Invited contribution to a convened session about "Leaky-Wave Antennas" at EuCAP 2012)

IC4. M. García-Vigueras, P. DeLara-Guarch, J.L. Gómez-Tornero, R. Guzmán-Quirós and G. Goussetis, "Efficiently illuminated broadside-directed 1D and 2D tapered Fabry-Perot leakywave antennas," Proceedings of the 6th European Conference on Antennas \& Propagation, (Prague, Czech Republic), pp.247-251, 26-30 March 2012 (Antenna Theory Best Paper Award).

IC5. R. Guzmán-Quirós, J. L. Gómez Tornero, M. García-Vigueras, A.R. Weily, Y. J. Guo, "Advances in electronically reconfigurable LWAs in Fabry-Pérot and SIW technologies", 7th European Conference on Antennas and Propagation (EUCAP 2013), pp. 224-228, April 2013. (Invited contribution to a convened session about "Emerging Antenna Technologies" at EuCAP 2013)

IC6. J. L. Gómez-Tornero, A. J. Martínez-Ros, R. Guzmán-Quirós, F. Quesada-Pereira, D. Cañete-Rebenaque, A. Álvarez-Melcón, "Theoretical and Applied Research at UPCT on the Modulation and Control of Leaky Waves in Substrate Integrated Waveguide Technology", 9th Iberian Meeting on Computational Electromagnetics (IX EIEC 2013), Dénia, Spain, May 2013.

IC7. A. J. Martínez-Ros, R. Guzmán-Quirós, J. L. Gómez Tornero, "Static and electronic shaping of the radiated electromagnetic fields in radial arrays of substrate integrated leaky-wave antennas," Microwave Conference (EuMC), 2013 European , vol., no., pp.1667,1670, 6-10 Oct. 2013. 


\subsubsection{Spanish Journals}

SJ1. R. Guzmán-Quirós, M. García Vigueras, A. J. Martínez Ros, J.L. Gómez Tornero, "Control electrónico del haz radiado en antenas leaky wave activas", IV Jornadas de Introducción a la Investigación (UPCT), Ed. UPCT, ISBN: 1888-8356, pp.94-96, May 2011.

SJ2. A. J. Martinez-Ros, M. García-Vigueras, R. Gúzman-Quiros and J. L. Gomez-Tornero, "Control del mecanismo de radiación de una antena leaky wave en tecnología planar", IV Jornadas de Introducción a la Investigación de la UPCT, Ed. UPCT, ISBN: 1888-8356, vol. 4, pp. 91-93, May 2011

SJ3. M. García-Vigueras, A. J. Martinez-Ros, R. Gúzman-Quiros and Gomez-Tornero, "Control de la directividad en broadside en antena leaky-wave 1D", IV Jornadas de Introducción a la Investigación de la UPCT, Ed. UPCT, ISBN: 1888-8356, vol. 4, pp. 97-99, May 2011

SJ4. R. Guzmán-Quirós, M. García Vigueras, J. L. Gómez Tornero, "Escaneo Electrónico Backward-a-Forward en Antenas Leaky-Wave 1D con Excitación Central", V Jornadas de Introducción a la Investigación (UPCT), Ed. UPCT, ISBN: 1888-8356, pp.102-104, April 2012.

SJ5. M. García Vigueras, R. Guzmán Quirós and J.L. Gómez Tornero, “Antenas Leaky-Wave 1D y 2D iluminadas eficientemente" V Jornadas de introducción a la investigación de la UPCT, Ed. UPCT, ISBN: 1888-8356, pp. 90-92, April 2012.

SJ6. R. Guzmán Quirós, A. J. Martínez Ros, J. L. Gómez Tornero, "Escaneo electrónico del haz de radiación en Antenas Leaky-Wave 2D empleando superficies de alta impedancia activas", VI Jornadas de Introducción a la Investigación de la UPCT (ISBN: 1888-8356), vol.6, pp.127-129, 2013.

SJ7. A. J. Martínez Ros, R. Guzmán Quirós, J. L. Gómez Tornero, “Aplicación de diseño Holográfico para reducir el nivel de lóbulo secundario en antenas leaky wave en tecnología SIW", VI Jornadas de Introducción a la Investigación de la UPCT (ISBN: 1888-8356),vol.6, pp.130-132, 2013.

SJ8. R. Guzmán Quirós, J. L. Gómez Tornero, "Diseño de una Antena Leaky-Wave Reconfigurable Electrónicamente en Tecnología de Guía de Onda Integrada en Sustrato", VII Jornadas de Introducción a la Investigación de la UPCT (ISBN: 1888-8356), vol.7, pp., 2014, Accepted. 


\subsubsection{Spanish Conference Proceedings}

SC1. M. García-Vigueras, R. Guzmán-Quirós, J.L. Gómez-Tornero , J.S. Gómez-Díaz, A. Álvarez-Melcón, "Analysis of Leaky modes in wave-guides wit parallel plates loaded with FSS and AMC applied to leaky-waves antennas", XXIV URSI (International Scientific Radiocommunications Union) Symposium, Santander (Cantabria), Spain, Sept. 2009.

SC2. M. García-Vigueras, J.L. Gómez-Tornero, R. Guzmán-Quirós, J.S. Gómez-Díaz, A.Álvarez-Melcón, "Control de la Radiación de una Antena Leaky-Wave Cargada con una Superficie Selectiva en Frecuencia”, XXV URSI (International Scientific Radiocommunications Union) Symposium, Bilbao (País Vasco), Spain, Sept. 2010.

SC3. R. Guzmán-Quirós, M. García-Vigueras, A. J. Martínez-Ros, J.L. Gómez-Tornero, "Reconfiguración Electrónica del Haz Radiado en Antenas Leaky Wave Basadas en HIS Activas", XXVI URSI (International Scientific Radiocommunications Union) Symposium, Madrid, Spain, Sept. 2011.

SC4. M. García-Vigueras, R. Guzmán-Quirós, A. Martínez-Ros, J.L. Gómez-Tornero, "Síntesis de la Radiación en Broadside en Antena Leaky-Wave 1D", XXVI URSI (International Scientific Radiocommunications Union) Symposium, Madrid, Spain, Sept. 2011.

SC5. A.J. Martínez-Ros, M. García-Vigueras, R. Guzmán-Quirós, J.L. Gómez-Tornero, "Determinación del Ángulo de Apuntamiento y de la Anchura de Haz en una Antena LeakyWave en Tecnología Planar" , XXVI URSI (International Scientific Radiocommunications Union) Symposium, Madrid, Spain, Sept. 2011.

SC6. R. Guzmán-Quirós, J. L. Gómez-Tornero, M. García-Vigueras, A. J. Martínez-Ros, "Backward-to-Forward Electronic Scanning in Symmetrically Excited 1D Fabry-Perot LeakyWave Antennas", XXVII URSI (International Scientific Radiocommunications Union) Symposium, Elche (Alicante), Spain, Sept. 2012.

SC7. A. J. Martinez-Ros, J. L. Gómez-Tornero, R. Guzmán-Quirós, M. García-Vigueras, "Holographic Pattern Synthesis With One-Dimensional Substrate Integrated Waveguide LeakyWave Antennas" , XXVII URSI (International Scientific Radiocommunications Union) Symposium, Elche (Alicante), Spain, Sept. 2012. (Second Prize Young Scientists URSI 2012)

SC8. M. García-Vigueras, J. L. Gómez-Tornero, R. Guzmán-Quirós, A. J. Martinez-Ros, "Optimización de la Directividad en Antenas Leaky-Wave 1D y 2D basadas en cavidades Fabry-Perot", XXVII URSI (International Scientific Radiocommunications Union) Symposium, Elche (Alicante), Spain, Sept. 2012. 
SC9. R. Guzmán Quirós, J. L. Gómez Tornero, A. J. Martínez Ros, Andrew R. Weily, Y. J. Guo, "Novel and Simple Electronically Reconfigurable Fabry-Pérot Antennas", XXVIII URSI (International Scientific Radiocommunications Union) Symposium, Santiago de Compostela, Spain, 11-13 Septiembre 2013. (First Prize Young Scientists URSI 2013)

SC10. A. J. Martínez-Ros, J. L. Gómez-Tornero, R. Guzmán-Quirós, "Nuevo Tipo de Circuito Multiplexador en Tecnología SIW Usando Técnicas Cuasi-Ópticas y Ondas de Fuga", XXVIII URSI (International Scientific Radiocommunications Union) Symposium, Santiago de Compostela, Spain, 11-13 Septiembre 2013. 


\subsubsection{Prizes}

The author has been recipient of three awards as author and co-author of three conference papers:

- SC9 has been recently awarded First Prize Young Scientists for being considered best student paper in the XVIII Spanish National Symposium of the International Union of Radio Science (URSI) celebrated in September 2013 in Santiago de Compostela (Spain).

- SC7 was awarded Second Prize Young Scientists in the XVII Spanish National Symposium of the International Union of Radio Science (URSI) celebrated in September 2012 in Elche (Spain).

- IC4 was awarded Best paper Award on Antenna Theory in the international $6^{\text {th }}$ European Conference on Antennas and Propagation (EuCAP) in March 2012. 

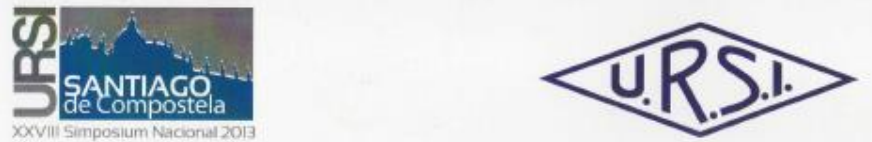

USC

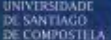

D. Eduardo Moreno Piquero, Vocal del Comité Organ zador de URSI 2013, D. Francisco José Ares Pena, Preside te del Comité Organizador de URSI 2013 y Presidente del Comité Nacional Español de URSI y D. Iuan Antonio Rodriguez González, Presidente del Comité Científico URSI 2013 y Secretario del Comité Nacional Español de URSI, otorgan el Premio Jóvenes Cientificos URSI 2013 a

Raúl Guzmán Quirós

como autor principal del artículo

Novel and Simple Electronically Reconfigurable Fabry-Pérot Antennas

Santiago de Compostela, a 12 de septiembre de 2013 Vocal del Comité Organizador de URSI 2013 

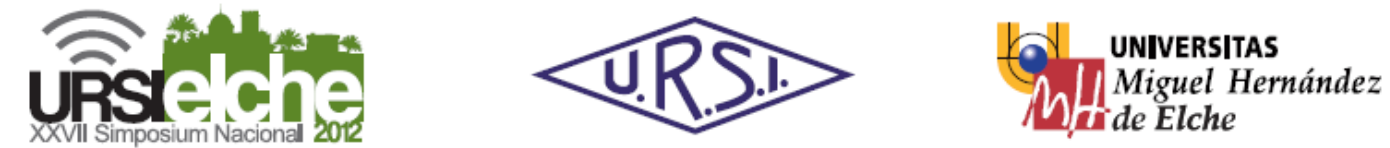

D. Enrique Bronchalo Bronchalo y D. Germán Torregrosa Penalva, Co-Presidentes del Comité Científico del XXVII Simposium Nacional de la Unión Científica Internacional de Radio, URSI 2012, D. Francisco José Ares Pena, Presidente del Comité Nacional Español de URSI y D. Juan Antonio Rodríguez González, Secretario del Comité Nacional Español de URSI, hacen constar que

\section{José Luis Gómez Tornero, Raúl Guzmán Quirós y María García Vigueras}

\section{son coautores del artículo}

“Holographic pattern synthesis with one-dimensional substrate integrated waveguide leaky-wave antennas" al que se ha otorgado el Accésit Jóvenes Científicos URSI 2012.

Elche, a 13 de septiembre de 2012.

Enrique Bronchalo Bronchalo Co-Presidente del Comité Científico de URSI 2012
Germán Torregrosa Penalva

Co-Presidente del Comité Científico de URSI 2012 


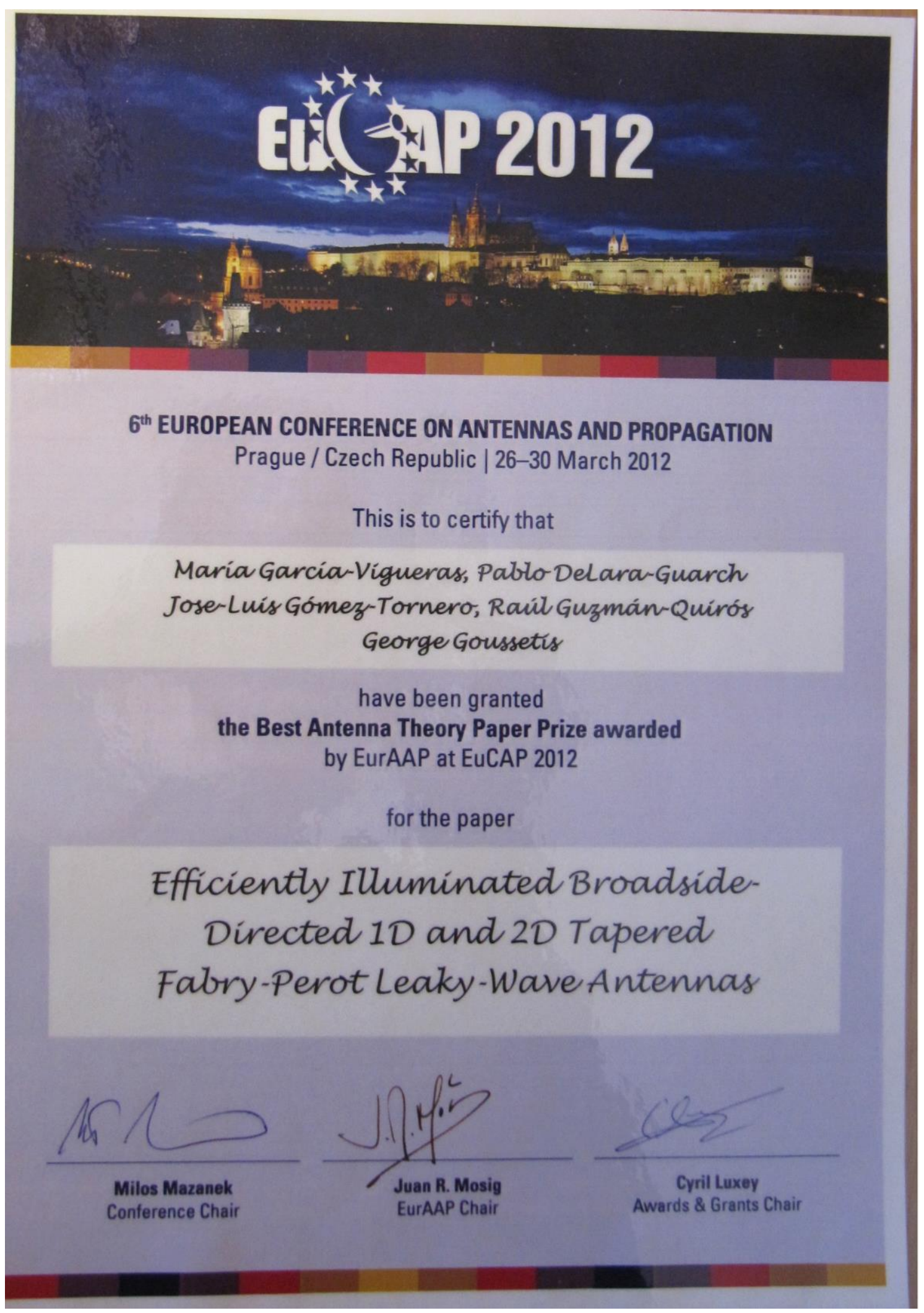




\subsection{Academic contributions}

The research work of this thesis has also given rise to one BSc. Thesis:

"Analysis of Leaky-Waves in Waveguides Loaded with Electronically Reconfigurable Frequency Selected Surfaces” (“Análisis De Ondas De Fuga En Guías De Onda Cargadas Con Superficies Selectivas En Frecuencia Reconfigurables Electrónicamente”), by Jesús Jiménez Campillo, Universidad Politécnica de Cartagena, 2010. Supervisors: José Luis Gómez Tornero and Raúl Guzmán Quirós. Grade A (10). 


\section{Bibliography}

[Aeroflex 2005] Aeroflex Microelectronic Solutions, "GaAs Hyperabrupt Varactor Diodes MGV Series," A17041 datasheet, Nov. 2005 [Online]. Available: www.aeroflexmetelics.com

[Alphones 1995] A. Alphones and M. Tsutsumi, "Leaky wave radiation from a periodically photoexcited semiconductor slab waveguide," IEEE Trans. Microwave Theory Tech.., vol. 43, pp. 2435-2441, Sept. 1995.

[Alphones 2013] Alphones, A; Mujumdar, M.; Cheng Jin, "Substrate integrated waveguide and its applications to leaky wave antennas," Microwave Conference Proceedings (APMC), 2013 Asia-Pacific, vol., no., pp.470,472, 5-8 Nov. 2013

[Archbold 2010] M. Archbold, E.J. Rothwell, L.C. Kempel, and S.W. Schneider, "Beam steering of a half-width microstrip leaky-wave antenna using edge loading," IEEE Antennas and Wireless Propag. Lett., vol. 9, pp. 203-206, 2010.

[Anagnostou 2006] D. E. Anagnostou, G. Zheng, M. T. Chryssomallis, J. C. Lyke, G. E. Ponchak, J. Papapolymerou, and C. G. Christodoulou, "Design, Fabrication, and Measurements of an RF-MEMS-based Self-Similar Reconfigurable Antenna," IEEE Trans. Antennas Propag., vol. 54, no. 2, pp. 422-432, February 2006

[Antoniades 2008] M. A. Antoniades, and G. V. Eleftheriades, "A CPS leaky-wave antenna with reduced beam squinting using NRI-TL metamaterials," IEEE Trans. Antennas Propag., vol. 56, no. 3, pp. 708-721, Mar. 2008.

[Augustin 2005] G. Augustin, S.V. Shynu, C.K. Aanandan, P. Mohanan, and K. Vasudevan, “A novel electronically scannable log-periodic leaky-wave antenna," Microw. and Opt. Techn. Lett., vol. 45, no. 2, pp. 163-165, April 2005.

[Azad 2005] A. K. Azad, W. Zhang, "Resonant terahertz transmission in subwavelength metallic hole arrays of sub-skin-depth thickness," Opt. Lett., vol. 30, no. 21, pp. 29452947, Nov. 2005.

[Bagby 1993] J. S. Bagby, L. Ching-Her, D. P. Nyquist, and Y. Yi, "Identification of propagation regimes on integrated microstrip transmission lines," IEEE Trans. Microw. Theory Tech., vol. 41, no. 11, pp. 1887-1894, 1993.

[Balanis 2005] C.A. Balanis, Antenna Theory, Singapore: John Wiley and Sons, $3^{\text {rd }}$ Ed.,2005, ch.2.8, “Antenna Efficiency”, pp.64-65. 
[Balcells 2010] J. Balcells, Y. Damgaci, B. A. Cetiner, J. Romeu, and L. Jofre, "Polarization reconfigurable MEMS-CPW antenna for mm-wave applications," in Proceedings of the 4th European Conference on Antennas and Propagation (EuCAP '10), April 2010.

[Barba 2007] M. Barba , E. Carrasco , J. E. Page and J. A. Encinar "Electronic controllable reflectarray elements in X band", Frequenz, J. RF-Eng. Telecomm., vol. 61, no. 910, pp.203-206, Oct. 2007

[Bernhard 2001] J. T. Bernhard, E. Kiely, and G. Washington, "A smart mechanically actuated two-layer electromagnetically coupled microstrip antenna with variable frequency, bandwidth, and antenna gain, "IEEE Transactions on Antennas and Propagation, vol. 49, no. 4, pp. 597-601, 2001.

[Bernhard 2005] J. T. Bernhard, K. Chang, Ed., "Reconfigurable antennas," in The Wiley Encyclopedia of RF and Microwave Engineering. New York: Wiley, Feb. 2005.

[Boccia 2010] L. Boccia, G. Amendola and G. Di Massa, "Performance improvement for a varactor-loaded reflectarray element", IEEE Trans. Antennas Propag., vol. 58, no. 2, pp.585-589 2010

[Boutayeb 2004] H. Boutayeb , T. Brillat, J. P. Daniel , F. Gadot , P. Y. Garel , A. de Lustrac , K. Mahdjoubi , P. Ratasjack and A. C. Tarot "A reconfigurable electromagnetic bandgap structure for a beam steering base station antenna", Proc. 27th ESA Antenna Technology Workshop on Innovative Periodic Antennas, pp.383 -389 2004

[Boutayeb 2006-I] H. Boutayeb, K. Mahdjoubi, A.-C. Tarot, and T. A. Denidni, "Directivity of an antenna embedded inside a Fabry-Pérot cavity: Analysis and design," Microw. Opt. Technol. Lett., vol. 48, pp. 12-17, Jan. 2006.

[Boutayeb 2006-II] H. Boutayeb, T. A. Denidni, K. Mahdjoubi, A. C. Tarot, A. R. Sebak, and L. Talbi, "Analysis and design of a cylindrical EBG based directive antenna," IEEE Trans. Antennas Propag., vol. 54, no. 1, pp. 211-219, Jan. 2006.

[Boutayeb 2006-III] H. Boutayeb, T.A: Denidni, "New Configuration of Cylindrical EBG Structure for Beam Switching Antennas," Antennas and Propagation Society International Symposium 2006, IEEE, vol., no., pp.2271-2274, 9-14 July 2006

[Boutayeb 2006-IV] H. Boutayeb, K. Mahdjoubi, "Dispersion Characteristics of a Cylindrical Electromagnetic Band Gap Structure", Microwave and Wireless Components Letters, IEEE, on page(s): 630 - 632 Volume: 16, Issue: 11, Nov. 2006

[Boutayeb 2007-I] H. Boutayeb, T. A. Denidni, and M. Nedil, "Bandwidth widening techniques for directive antennas based on partially reflecting surfaces," Progress In Electromagnetics Research, Vol. 74, 407-419, 2007. 
[Boutayeb 2007-II] H. Boutayeb and T. A. Denidni, "Metallic Cylindrical EBG Structures With Defects: Directivity Analysis and Design Optimization," Antennas and Propagation, IEEE Transactions on, vol.55, no.11, pp.3356, 3361, Nov. 2007

[Burghignoli 2003] P. Burghignoli, F. Frezza; A. Galli and G. Schettini, "Synthesis of broadbeam patterns through leaky-wave antennas with rectilinear geometry," Antennas and Wireless Propagation Letters, IEEE, vol.2, no.1, pp.136, 139, 2003

[Burghignoli 2006] P. Burghignoli, G. Lovat, and D. R. Jackson, "Analysis and optimization of leaky-wave radiation at broadside from a class of 1- D periodic structures," IEEE Trans. Antennas Propag., vol. 54, no. 9, pp. 2593-2603, Sep. 2006.

[Burghignoli 2008] P. Burghignoli, G. Lovat, F. Capolino, D. R. Jackson, and D. R. Wilton, "Directive leaky-wave radiation from a dipole source in a wire-medium slab," IEEE Trans. Antennas Propag., vol. 56, no. 5, pp. 1329-1339, May 2008.

[Burokur 2013] S.N. Burokur, G. Piau, G. Sabanowski, A. de Lustrac, "Active metasurface for low-profile reconfigurable antennas," Antennas and Propagation (EuCAP), 2013 7th European Conference on, vol., no., pp.1649, 1652, 8-12 April 2013

[Bruni 2007] S. Bruni, A. Neto, F. Marliani, "The Ultrawideband Leaky Lens Antenna," Antennas and Propagation, IEEE Transactions on, vol.55, no.10, pp.2642,2653, Oct. 2007

[Cai 2012] Y. Cai, Y. J. Guo, and T. S. Bird, "A Frequency reconfigurable printed yagi-Uda dipole antenna for cognitive radio applications," IEEE Trans. Antennas Propag., Vol. 60, No. 6, pp. 2905-2912, Jun. 2012.

[Carrasco 2012-I] E. Carrasco, M. Barba, B. Reig, C. Dieppedale, J.A. Encinar, "Characterization of a Reflectarray Gathered Element With Electronic Control Using Ohmic RF MEMS and Patches Aperture-Coupled to a Delay Line," Antennas and Propagation, IEEE Transactions on, vol.60, no.9, pp.4190,4201, Sept. 2012

[Carrasco 2012-II] E. Carrasco, M. Barba, J.A. Encinar, "X-Band Reflectarray Antenna With Switching-Beam Using PIN Diodes and Gathered Elements," Antennas and Propagation, IEEE Transactions on, vol.60, no.12, pp.5700,5708, Dec. 2012

[Carrasco 2012-III] E. Carrasco, J. A. Encinar, and J. Perruisseau-Carrier, "Evaluation of a reflectarray with independent scanning of two linearly-polarized beams," in Proc. 6th Eur. Conf. Antennas Propag. (EUCAP), 2012, pp. 2967-2970.

[Carrasco 2013] E. Carrasco, M. Tamagnone, J. Perruisseau-Carrier, "Tunable graphene reflective cells for THz reflectarrays and generalized law of reflection," Applied Physics Letters , vol.102, no.10, pp.104103,104103-4, Mar 2013 
[Caloz 2004] C. Caloz, T. Itoh, "Array factor approach of leaky-wave antennas and application to 1-D/2-D composite right/left-handed (CRLH) structures," Microwave and Wireless Components Letters, IEEE, vol.14, no.6, pp.274,276, June 2004

[Caloz 2005] C. Caloz and T. Itoh "Electromagnetic Metamaterials: Transmission Line Theory and Microwave Applications", 2005 :Wiley-IEEE

[Cetiner 2010] B. A. Cetiner, G. R. Crusats, L. Jofre, and N. Biyikli, "RF MEMS integrated frequency reconfigurable annular slot antenna," IEEE Trans. Antennas Propag., vol. 58, no. 3, pp. 626-632, Mar. 2010.

[Chang 1999] B. C. Chang, Y. Qian, T. Itoh, "A reconfigurable leaky mode/patch antenna controlled by PIN diode switches," Antennas and Propagation Society International Symposium, 1999. IEEE, vol.4, no., pp.2694-2697 vol.4, 11-16 July 1999.

[Chang 2012] R.Y. Chang, and F.C. Chen, "Leaky-wave antenna capable of multi-plane scanning," Google Patents, US Patent 8,253,642, August 2012 (available online: http://www.google.com/patents/US8253642)

[Chen 2002] C.-C. Chen and C.-K.C Tzuang, "Phase-shifterless beam-steering micro-slotline antenna," Electron. Lett., vol.38, no.8, pp. 354 - 355, April 2002.

[Chicherin 2006] D. Chicherin, S. Dudorov, D. Lioubtchenko, V. Ovchinnikov, S. Tretyakov, and A.V. Räisänen, "MEMS-based high-impedance surfaces for millimeter and submillimeter wave applications," Microwave and Optical Technology Letters, vol. 48, no. 12, pp. 2570-2573, 2006.

[Chicherin 2011-I] D. Chicherin, M. Sterner, D. Lioubtchenko, J. Oberhammer, and A.V. Räisänen, “Analog-type millimeter wave phase shifters based on MEMS tunable highimpedance surface and dielectric rod waveguide," Int. J. of Microwave and Wireless Technologies, vol. 3, pp. 533-538, 2011.

[Chicherin 2011-II] D. Chicherin, "Studies on microelectromechanically tuneable highimpedance surface for millimeter wave beam steering," Doctoral dissertation, Aalto University, Department of Radio Science and Engineering, 2011.

[Christodoulou 2012] C.G, Christodoulou, Y. Tawk, S.A. Lane, S.R. Erwin, "Reconfigurable Antennas for Wireless and Space Applications," Proceedings of the IEEE, vol.100, no.7, pp.2250,2261, July 2012

[Clark 2003] R. Clark, G. Huff, and J. Bernhard, "An integrated active microstrip reflectarray element with an internal amplifier," IEEE Trans. Antennas Propag., vol. 51, no. 5, pp. 993-999, May 2003. 
[Clarricoats 1991] P. J. B. Clarricoats, H. Zhou, and A. Monk, "Electronically Controlled Reconfigurable Reflector Antenna," Proc. IEEE Int. Symp. on Antennas and Propagation, vol. 1 (June 1991): 179-181.

[Costantine 2009] J. Costantine, "Design, optimization and analysis of reconfigurable antennas", Ph.D. dissertation, Electr. Comput. Eng. Dept., Univ. New Mexico (UNM), Albuquerque, NM, Dec. 2009.

[Costa 2008] F. Costa, A. Monorchio, S. Talarico, and F. M. Valeri, "A tuneable high impedance surface for low profile tuneable and steerable antennas," IEEE Antennas and Wireless Propagation Letters, vol. 7, pp. 676-680, 2008.

[Costa 2009] F. Costa, S. Genovesi, and A. Monorchio, "On the Bandwidth of High-Impedance Frequency Selective Surfaces," IEEE Antennas Wireless and Propagation Letters, 8 , 2009, pp.1341-1344.

[Costa 2010] F. Costa, A. Monorchio, G. Manara, "Analysis and design of ultra-thin electromagnetic absorbers comprising resistively loaded high impedance surfaces," IEEE Trans. Antennas Propag, vol. 58, no. 5, pp. 1551, 2010.

[Costa 2011] F. Costa and A. Monorchio, "Design of subwavelength tuneable and steerable Fabry-Perot leaky-wave antennas,"Progress In Electromagnetics Research, vol. 111, pp. 467-481, 2011.

[Costa 2012] F. Costa, A. Monorchio, G. Manara, "Efficient Analysis of Frequency-Selective Surfaces by a Simple Equivalent-Circuit Model," Antennas and Propagation Magazine, IEEE, vol.54, no.4, pp.35,48, Aug. 2012

[CST 2010] CST, “Computer Simulation Technology”, v.2010.O1.

[Debogovic 2010] T. Debogovic, J. Perruisseau-Carrier, and J. Bartolic, "Partially reflective surface antenna with dynamic beamwidth control," IEEE Antennas Wireless Propag. Lett., vol. 9, pp. 1157-1160, 2010.

[Debogovic 2014-I] T. Debogovic, J. Bartolic, J. Perruisseau-Carrier, "Dual-Polarized Partially Reflective Surface Antenna With MEMS-Based Beamwidth Reconfiguration," Antennas and Propagation, IEEE Transactions on, vol.62, no.1, pp.228,236, Jan. 2014

[Debogovic 2014-II] T. Debogovic, J. Bartolic, and J. Perruisseau-Carrier, "Array-Fed Partially Reflective Surface Antenna With Independent Scanning and Beamwidth Dynamic Control", Antennas and Propagation, IEEE Transactions on, On page(s): 446 - 449 Volume: 62, Issue: 1, Jan. 2014 
[Deslandes 2001] D. Deslandes and K. Wu, "Integrated microstrip and rectangular waveguide in planar form," IEEE Microw. Wireless Compon. Lett., vol. 11, no. 2, pp. 68 -70, Feb. 2001.

[Deslandes 2002] D. Deslandes and K. Wu, "Design consideration and performance analysis of substrate integrated waveguide components," in Microwave Conference, 2002. 32nd European, Sep. 2002, pp. $1-4$.

[Deslandes 2005] D. Deslandes and K.Wu, "Substrate integrated waveguide leaky-wave antenna: concept and design considerations", in APMC 2005. Asia-Pacific Microwave Conf. Proc., Suzhou, China, Dec. 2005, pp. 346 -349.

[Djerafi 2008] T. Djerafi, K. Wu; H. Boutayeb, "Electronically steerable antenna using a circular patch surrounded by a reconfigurable circular mushroom-like substrate," Microwave Conference, 2008. APMC 2008. Asia-Pacific, vol., no., pp.1,4, 16-20 Dec. 2008

[Doumanis 2012] E. Doumanis , G. Goussetis , J. L. Gomez-Tornero , R. Cahill and V. Fusco "Anisotropic impedance surfaces for linear to circular polarization conversion", IEEE Trans. Antennas Propag., vol. 60, no. 1, pp.212 -219 2012

[Doumanis 2014] Doumanis, E.; Goussetis, G.; Dickie, R.; Cahill, R.; Baine, P.; Bain, M.; Fusco, V.; Encinar, J.A; Toso, G., "Electronically Reconfigurable Liquid Crystal Based Mm-Wave Polarization Converter," Antennas and Propagation, IEEE Transactions on , vol.62, no.4, pp.2302,2307, April 2014

[Duran-Sindreu 2013] Duran-Sindreu, M.; Jun Choi; Bonache, J.; Martin, F.; Itoh, T., "Dualband leaky wave antenna with filtering capability based on extended-composite right/left-handed transmission lines," Microwave Symposium Digest (IMS), 2013 IEEE MTT-S International , vol., no., pp.1,4, 2-7 June 2013

[Edalati 2009] A. Edalati, T.A. Denidni, "Reconfigurable Beamwidth Antenna Based on Active Partially Reflective Surfaces," Antennas and Wireless Propagation Letters, IEEE, vol.8, no., pp.1087,1090, 2009

[Edalati 2011] A. Edalati, T.A. Denidni, "High-Gain Reconfigurable Sectoral Antenna Using an Active Cylindrical FSS Structure", Antennas and Propagation, IEEE Transactions on, On page(s): 2464 - 2472 Volume: 59, Issue: 7, July 2011

[Eleftheriades 2005] G.V. Eleftheriades and K.G. Balmain, Negative-Refraction Metamaterials: Fundamental Principles and Applications. Wiley \& IEEE Press, Hoboken, NJ. editors (2005). 
[Engheta 2002] N. Engheta, "Thin absorbing screens using metamaterial surfaces," in Proc. IEEE Antennas Propagation Soc. Int. Symp., 2002, vol. 2, pp. 392-395.

[Esquius-Morote 2014] Esquius-Morote, M.; Gomez-Diaz, J.S.; Perruisseau-Carrier, J., "Sinusoidally Modulated Graphene Leaky-Wave Antenna for Electronic Beamscanning at THz," Terahertz Science and Technology, IEEE Transactions on , vol.4, no.1, pp.116,122, Jan. 2014

[Ettorre 2007] M. Ettorre, S. Bruni, G. Gerini, A. Neto, N. Llombart, S. Maci, "Sector PCSEBG Antenna for Low Cost High Directivity Applications", Antennas and Wireless Propagation Letters, Vol.6, pp. 537-539, Dec. 2007.

[Ettorre 2008] M. Ettorre, A. Neto, G. Gerini, and S. Maci, "Leaky-wave slot array antenna fed by a dual reflector system," IEEE Trans. Antennas Propag., vol. 56, no. 10, pp. 31433149, Oct. 2008

[Ettorre 2011] M. Ettorre, R. Sauleau L. Le Coq, "Multi-Beam Multi-Layer Leaky-Wave SIW Pillbox Antenna for Millimeter-Wave Applications," Antennas and Propagation, IEEE Transactions on , vol.59, no.4, pp.1093,1100, April 2011

[Fathy 2003] A. Fathy, A. Rosen, H. Owen, F. McGinty, D. McGee, G. Taylor, R. Amantea, P. Swain, S. Perlow, and M. ElSherbiny, "Silicon-based reconfigurable antennas-concepts, analysis, implementation, and feasibility," Microwave Theory and Techniques, IEEE Transactions on, vol.51, no.6, pp.1650,1661, June 2003

[Feresidis 2005] A.P. Feresidis, G. Goussetis, S. Wang and J.C. Vardaxoglou, "Artificial magnetic conductor surfaces and their application to low-profile high-gain planar antennas," IEEE Trans. Antennas Propag., vol. 53, no. 1, pp. 209-215, Jan. 2005.

[Feresidis 2006] Feresidis, A.P.; Vardaxoglou, J.C., "A broadband high-gain resonant cavity antenna with single feed," Antennas and Propagation, 2006. EuCAP 2006. First European Conference on , vol., no., pp.1,5, 6-10 Nov. 2006

[Fralich 1992] R. Fralich and J. Litva, "Beam-steerable tuneable array antenna," Electron. Lett., vol. 28, pp. 184-185, Jan. 1992.

[Freeman 1992] J. L. Freeman, B. J. Lamberty, and G. S. Andrews, "Optoelectronically Reconfigurable Monopole Antenna," Electron. Lett., vol. 28, no. 16, pp.1502-1503, July 1992

[García-Vigueras 2010] M. García-Vigueras, J.L. Gómez-Tornero, G. Goussetis, J.S. GómezDíaz, and A. Álvarez-Melcón, "A modified pole-zero technique for the synthesis of waveguide leaky-wave antennas loaded with dipole-based FSS," IEEE Trans. Antennas Propagat., vol. 58, no. 6, pp. 1971-1979, June 2010. 
[García-Vigueras 2011-I] M. García-Vigueras, J.L. Gómez-Tornero, G. Goussetis, Andrew R. Wiley, and Y. Jay Guo, "Enhancing frequency-scanning response of leaky-wave antennas using high impedance surfaces," IEEE Antennas and Wireless Propag. Lett., vol. 10, pp. 7-10, March 2011.

[García-Vigueras 2011-II] M. García-Vigueras, J.L. Gómez-Tornero, G. Goussetis, A. R. Weily, and Y.J. Guo, "1D-leaky wave antenna employing parallel-plate waveguide loaded with PRS and HIS," IEEE Trans. Antennas Propag., vol. 59, no. 10, pp. 3687 - 3694, Oct. 2011.

[García-Vigueras 2012-I] M. Garcia-Vigueras, F. Mesa, F. Medina, R. Rodriguez-Berral, J.L Gomez-Tornero., "Simplified Circuit Model for Arrays of Metallic Dipoles Sandwiched Between Dielectric Slabs Under Arbitrary Incidence," Antennas and Propagation, IEEE Transactions on, vol.60, no.10, pp.4637,4649, Oct. 2012

[García-Vigueras 2012-II] María García-Vigueras,” Analysis and Design of Hybrid LeakyWave Antennas Loaded with Frequency Selective Surfaces", Technical University of Cartagena, Supervisor: José Luis Gómez Tornero, October 2012, available online : http://repositorio.bib.upct.es/dspace/handle/10317/3197

[Goldstone 1959] L. Goldstone, and A.A Oliner, "Leaky-wave antennas I: Rectangular waveguides," IRE Transactions on Antennas and Propagation, vol. 7, no. 4, pp. 307 319, April 1959.

[Gómez 2005-I] J.L. Gómez, Analisis de modos de fuga en estructuras planares apantalladas lateralmente y diseño de nuevas antenas "leaky-wave" en tecnologia hibrida impresaapantallada [Dr. dissertation]. Spain: Universidad Politecnica de Cartagena (Spain); 2005 In: Dissertations \& Theses @ Universidad Politecnica de Cartagena [database on the Internet]. Available from: http://www.proquest.com/; Publication Number: AAT 3388885, ISBN: 978-11-0955-477-9; Source: Dissertation Abstracts International, Volume: 71-01, Section: B, page: 0534.; 466 páginas, May 2005.

[Gómez 2005-II] J. L. Gómez, D. Cañete and A. Álvarez-Melcón, "Printed-circuit leaky-wave antenna with pointing and illumination flexibility", IEEE Microw.WirelessCompon. Lett.,vol. 15, no. 8, pp. 536-538, Aug. 2005.

[Gómez 2006-I] J. L. Gómez, J. Pascual and A. Álvarez-Melcón, “Efficient Full-Wave Analysis Method of Leaky-Wave Modes in Periodically Loaded Dielectric Waveguides with Application to Backward-to-Forward Frequency-Scannable Antennas and Metamaterials", Int. Journal of Numerical Modelling: Special Issue on Numerical Modelling of Metamaterial Properties, Structures and Devices., Vol.19, No.2, pp.173193, March 2006. 
[Gómez 2006-II] J.L. Gómez, D. Cañete, F. Quesada, J. Pascual and A.A. Melcón, "P.A.M.E.L.A: A Useful Tool for the Study of Leaky-Wave Modes in Strip-Loaded Open Dielectric Waveguides", IEEE Antennas and Propagat. Magazine, Vol.48, No.4, pp.54-72, August 2006.

[Gómez 2007] J.L. Gómez, G. Goussetis and A.A. Melcón, "Correction of dielectric losses in leaky-wave antenna designs," Journal of Electromagnetic Waves and Applications, vol. 21, no. 8, pp. 1025-1036, 2007.

[Gómez 2010] J.L. Gomez-Tornero , A. Martinez-Ros and R. Verdu-Monedero "FFT synthesis of radiation patterns with wide nulls using tapered leaky-wave antennas", IEEE Antennas Wireless Propag. Lett., vol. 9, pp.518 -521 2010

[Gómez 2011-I] J.L Gomez-Tornero., "Unusual tapering of leaky-wave radiators and their applications," Antennas and Propagation (EUCAP), Proceedings of the 5th European Conference on, vol., no., pp.821,824, 11-15 April 2011

[Gómez 2011-II] J.L Gomez-Tornero, "Analysis and Design of Conformal Tapered LeakyWave Antennas," Antennas and Wireless Propagation Letters, IEEE, vol.10, no., pp.1068,1071, 2011

[Gómez 2011-III] J.L. Gomez-Tornero, A. Weily and Y. Guo, "Rectilinear leaky-wave antennas with broad beam patterns using hybrid printed-circuit waveguides", IEEE Trans. Antennas Propag., vol. 59, no. 11, pp.3999 -4007 2011

[Gómez 2013-I] J.L. Gomez-Tornero, A. Martinez-Ros, A. Alvarez-Melcon, F. Mesa, F. Medina, "Substrate integrated waveguide leaky-wave antenna with reduced beam squint," Microwave Conference (EuMC), 2013 European , vol., no., pp.491,494, 6-10 Oct. 2013

[Gómez 2013-II] J.L. Gomez-Tornero, D. Blanco , E. Rajo-Iglesias and N. Llombart "Holographic surface leaky-wave lenses with circularly-polarized focused near-fields. Part I: Concept, design and analysis theory", IEEE Trans. Antennas Propag., 2013

[Goussetis 2006-I] G. Goussetis, A.P. Feresidis, J.C. Vardaxoglou, "Tailoring the AMC and EBG Characteristics of Periodic Metallic Arrays Printed on Grounded Dielectric Substrate," IEEE Transactions Antennas and Propagation, Vol. 54, No. 1, Jan. 2006, pp. 82-89.

[Goussetis 2006-II] G. Goussetis, A.P. Feresidis and P. Kosmas, "Efficient Analysis, Design and Filter Applications of EBG Waveguide with Periodic Resonant Loads," IEEE Trans. Microw. Theory Tech., Vol. 54, No. 11, pp. 3885-3892, November 2006. 
[Goussetis 2007-I] G. Goussetis, N. Uzunoglou, J.-L. Gomez-Tornero, B. Gimeno, V.E. Boria, "An E-plane EBG Waveguide for Dispersion Compensated Transmission of Short Pulses”, IEEE Antenna and Propagation Symp. Dig., Honolulu, 9-15 June 2007.

[Goussetis 2007-II] G. Goussetis, A.P. Feresidis and R. Cheung, "Quality factor Assessment of Subwavelength Cavities at FIR Frequencies," Journal of Optics A, Vol. 9, pp. s355s360, August 2007.

[Goussetis 2010] G. Goussetis, J.L Gómez-Tornero, A.P. Feresidis, and N. Uzunoglu, "Artificial impedance surfaces for reduced dispersion in antenna feeding systems", IEEE Trans. Antennas Propag., vol.58, no. 11, pp. 3629-3636, Nov. 2010.

[Guclu 2012] C. Guclu, J. Perruisseau-Carrier, and O. Civi, "Proof of concept of a dual-band circularly-polarized RF MEMS beam-switching reflectarray," IEEE Trans. Antennas Propag., vol. 60, no. 11, pp. 5451-5455,2012.

[Guerci, 2014] J.R. Guerci, et al, "Next Generation Affordable Smart Antennas", Microwave Journal, Jan. 2014 (http://www.microwavejournal.com/articles/21277-next-generationaffordable-smart-antennas)

[Gupta 2000] K. C. Gupta, J. Li, R. Ramadoss, and C. Wang, "Design of FrequencyReconfigurable Slot Ring Antennas," Proc. IEEE/URSI Int. Symp on Antennas and Propagation, vol. 1 pp. 326, July 2000

[Grbic 2002] A. Grbic, and G.V. Eleftheriades, "Leaky CPW-based slot antenna arrays for millimeter-wave applications", IEEE Trans. Antennas Propag., vol.50, no.11, pp. 1494 - 1504, Nov. 2002.

[Haider, 2013] N. Haider, D. Caratelli, and A. G. Yarovoy, "Recent Developments in Reconfigurable and Multiband Antenna Technology", Microwave Journal, Jan. 2013 (http://dx.doi.org/10.1155/2013/869170)

[Hansen 1940] W. W. Hansen, "Radiating electromagnetic waveguide," Patent, 1940,U.S. Patent No. 2.402.622.

[Hansen 1998] R.C. Hansen, Phased Array Antennas, John Wiley \& Sons, New York, 1998.

[Hessel 1969] A. Hessel, Antenna Theory, Part II, R. E. Collin and R. F. Zucker, Eds. New York: McGraw-Hill, 1969, chap. 19.

[HFSS 2011] Ansoft Corporation, "High Frequency Structure Simulator (HFSS)”, v.11.

[Hines 1957] J. N. Hines and J. R. Upson, "A Wide Aperture Tapered-Depth Scanning Antenna," Ohio State Univ. Res. Found., Report 667-7, Columbus, OH, December 1957. 
[Honey 1959] R. C. Honey, "A flush-mounted leaky-wave antenna with predictable patterns," IRE Transactions on Antennas and Propagation, vol. 7, no. 4, pp. 320 - 329, April 1959.

[Hong 2007] W. Hong, B. Liu, G. Luo, Q. Lai, J. Xu, Z. Hao, F. He, and X. Yin, “Integrated microwave and millimeter wave antennas based on siw and hmsiw technology," in Antenna Technology: Small and Smart Antennas Metamaterials and Applications, 2007. IWAT ’07. International Workshop on, Mar. 2007, pp. 69 -72.

[Horn 1980] R. E. Horn, H. Jacobs, E. Freibergs, and K. L. Klohn, "Electronic modulated beam steerable silicon waveguide array antenna," IEEE Trans. Microwave Theory Tech., vol.28, pp. 647-653, June 1980.

[Hu 1998] C. C. Hu, C. F. Jou, and J. J. Wu, "Two-dimensional beam-scanning phaseshifterless technique using linear active leaky-wave antenna array," IEE Electron. Lett., vol. 34, no. 13, pp. 1282-1283, Jun. 1998.

[Hu 1999] C. C. Hu, C. F. Jou, and J. J. Wu, "A two-dimensional beam-scanning linear active leaky-wave antenna array," IEEE Microw. Guided Wave Lett., vol. 9, no. 3, pp. 102104, Mar. 1999.

[Hu 2000-I] C.-C.Hu, C.F. Jou, and J.-J. Wu, "Two-dimensional beam-scanning linear tuneable leaky-wave antenna array using coupled VCOs," IEE Proc. Microw., Antennas and Propag., vol.147, no.1, pp. 68 - 72, Feb. 2000.

[Hu 2000-II] C. C. Hu, C. F. Jou, and J. J. Wu, “An aperture-coupled linear microstrip leakywave antenna array with two-dimensional dual-beam scanning capability," IEEE Trans. Antennas Propag., vol. 48, no. 6, pp. 909-913, Jun. 2000.

[Hu 2008] W. Hu, R. Cahill, J. Encinar, R. Dickie, H. Gamble, V. Fusco, and N.Grant, "Design and measurement of reconfigurable millimeter wave reflectarray cells with nematic liquid crystal," IEEE Trans. Antennas Propag., vol. 56, no. 10, pp. 3112-3117, 2008.

[Huang 2008] J. Huang and J. A. Encinar, "Reflectarray Antennas", 2008: IEEE Press, Wiley

[Huang 2000] L. Huang, J. Chiao, and P. Lisio, "An electronically switchable leaky wave antenna," IEEE Trans. Antennas Propagat., vol. 48, pp.1769-1772, Nov. 2000.

[Huff 2005] G. H. Huff and J. T. Bernhard, "Frequency Reconfigurable CPW-fed Hybrid Folded Slot/Slot Dipole Antenna," Proc. IEEE/ACES Int. Conf. on Wireless Communications and Applied Computational Electromagnetics, pp. 574-577, April 2005 
[Huff 2006] G. H. Huff and J. T. Bernhard, "Integration of packaged RF-MEMS switches with radiation pattern reconfigurable square spiral microstrip antennas," IEEE Trans. Antennas Propag., vol. 54, no. 2, pp. 464-469, Feb. 2006.

[Hum 2005] S. V. Hum, M. Okoniewski, and R. J. Davies, "Realizing an electronically tuneable reflectarray using varactor diode-tuned elements," IEEE Microw.Wireless Compon. Lett., vol. 15, no. 6, pp. 422-424, June 2005.

[Hum 2007] S. V. Hum, M. Okoniewski, and R. J. Davies, "Modeling and design of electronically tunable reflectarrays," IEEE Trans. Antennas Propag., vol. 55, no. 8, pp. 2200-2210, Aug. 2007.

[Hum 2010] S. V. Hum and H. Y. Xiong, "Analysis and design of a differentially-fed frequency agile microstrip patch antenna,"IEEE Transactions on Antennas and Propagation, vol. 58, no. 10,pp. 3122-3130, 2010.

[Hum 2014] S.V. Hum, J. Perruisseau-Carrier, "Reconfigurable Reflectarrays and Array Lenses for Dynamic Antenna Beam Control: A Review," Antennas and Propagation, IEEE Transactions on , vol.62, no.1, pp.183,198, Jan. 2014

[Ip 1990] A. Ip and D.R. Jackson, "Radiation from cylindrical leaky waves," IEEE Trans. Antennas Propagat., vol. 38, no. 4, pp.482-488, Apr 1990.

[Jackson 1985] D. Jackson, and N. Alexopoulos,"Gain enhancement methods for printed circuit antennas," IEEE Trans. Antennas Propag., vol.33, no.9, pp. 976- 987, Sep 1985.

[Jackson 1988] D. R. Jackson and A.A. Oliner, "A leaky-wave analysis of the high gain printed antenna configuration," IEEE Trans. Antennas Propag., vol. 36, no. 7, pp. 905-910, Jul. 1988.

[Jackson 1993] D. R. Jackson, A.A. Oliner and A. Ip, "Leaky-wave propagation and radiation for a narrow-beam multiple-layer dielectric structure," IEEE Trans. Antennas Propag., vol.41, no.3, pp.344-348, Mar 1993.

[Jackson 2008] D. R. Jackson and A.A. Oliner, "Leaky-wave antennas," in Modern Antenna Handbook, C. A. Balanis, ed. (Wiley, 2008), pp. 325-367.

[Jackson 2011] D. R. Jackson, P. Burghignoli, G. Lovat, F. Capolino, J. Chen, D. R. Wilton, and A.A. Oliner, "The Fundamental Physics of Directive Beaming at Microwave and Optical Frequencies and the Role of Leaky Waves," Proceedings of the IEEE, Vol. 99, No. 10, pp.1780-1805,2011. 
[Jung 2006] C. W. Jung, M. Lee, G. P. Li, and F. De Flaviis, "Reconfigurable scan-beam singlearm spiral antenna integrated with RF-MEMS switches," IEEE Trans. Antennas Propag., vol. 54, no. 2, pp. 455-463, Feb. 2006.

[Karmokar 2013] D. K. Karmokar, D. N. P. Thalakotuna, K. P. Esselle, L. Matekovits, and M. Heimlich, "Reconfigurable Half-Width Microstrip Leaky-Wave Antenna for FixedFrequency Beam Scanning," Proceedings of 7th European Conference on Antennas and Propagation (EuCAP 2013), 8 - 12 April 2013, Gothenburg, Sweden

[Kelly 2007] J.R. Kelly et al., "Dual Frequency Leaky-Wave Antenna Analysis and Design," Antennas and Propagation, 2007. EuCAP 2007. The Second European Conference on, vol., no., pp.1, 5, 11-16 Nov. 2007

[Kallel 2013] A. Kallel, J. Sokoloff, T. Callegari, "Theory and simulations of a beam-scanning plasma antenna," Antennas and Propagation (EuCAP), 2013 7th European Conference on, vol., no., pp.3457, 3461, 8-12 April 2013

[King 2013] A.J. King, J.F. Patrick, N.R. Sottos, S.R. White, G.H. Huff, J.T. Bernhard, "Microfluidically Switched Frequency-Reconfigurable Slot Antennas," Antennas and Wireless Propagation Letters, IEEE, vol.12, no., pp.828,831, 2013

[Kodera 2009] T. Kodera, C. Caloz, "Dual-Band Full-Space Scanning Leaky-Wave Antenna Based on Ferrite-Loaded Open Waveguide," Antennas and Wireless Propagation Letters, IEEE, vol.8, no., pp.1202,1205, 2009

[Korosec 2006] T. Korosec, P. Ritosa, and M. Vidmar, "Varactor-tuned microstrip-patch antenna with frequency and polarization agility," Electronics Letters, vol. 42, no. 18, pp. 1015-1016, 2006.

[Krauss 2011] A. Krauss, H. Bayer, R. Stephan, M.A. Hein, "A dual-band circularly-polarised leaky-wave antenna for mobile Ka-band satellite communications," Antennas and Propagation in Wireless Communications (APWC), 2011 IEEE-APS Topical Conference on, vol., no., pp.203,206, 12-16 Sept. 2011

[Lampariello 1987] P. Lampariello, A.A. Oliner, "A Novel Phased Array of Printed-Circuit Leaky-Wave Line Sources," Microwave Conference, 1987. 17th European, vol., no., pp.555, 560, 7-11 Sept. 1987

[Lau 2012] J. Y. Lau and S. V. Hum, "Reconfigurable transmitarray design approaches for beamforming applications," IEEE Trans. Antennas Propag., vol. 60, no. 12, pp. 56795689, 2012. 
[Lee 2007] D. Lee, S. Lee, C. Cheon and Y. Kwon "A Two-Dimensional Beam Scanning Antenna Array Using Composite Right/Left Handed Microstrip Leaky-Wave Antennas", Proc. Int. Microwave Symposium (IMS), pp.1883 -1886 2007

[Li 2006] Y. Li and Y. Long, "Frequency-fixed beam-scanning microstrip leaky-wave antenna with multi-terminals," Electron. Lett., vol. 42, no. 1, pp. 7-8, Jan. 2006.

[Li 2008-I] Y. Li, Q. Xue, E.K.-N. Yung, and Y. Long, "Dual-beam steering microstrip leaky wave antenna with fixed operating frequency," IEEE Trans. Antennas Propag., vol.56, no.1, pp. 248-252, Jan. 2008.

[Li 2008-II] Y. Li, Q. Xue, E.K.-N. Yung, Y. Long, "Two-dimensional beam-scanning microstrip leaky-wave antenna," Electronics Letters, vol.44, no.3, pp.183, 185, January 312008

[Li 2009] Y. Li, Q. Xue, E.K.-N. Yung, Y. Long, "Quasi Microstrip Leaky-Wave Antenna With a Two-Dimensional Beam-Scanning Capability," Antennas and Propagation, IEEE Transactions on, vol.57, no.2, pp.347, 354, Feb. 2009

[Lim 2004-I] S. Lim, C. Caloz, and T. Itoh, "Electronically scanned composite right/left handed microstrip leaky-wave antenna," IEEE Microwave and Wireless Components Lett., vol. 14, no. 6, pp. 277 - 279, June 2004.

[Lim 2004-II] S. Lim, C. Caloz, and T. Itoh, "Metamaterial-based electronically controlled transmission-line structure as a novel leaky-wave antenna with tuneable radiation angle and beamwidth", IEEE Trans. Antennas Propag., vol.52, no.12, pp. 2678-2690, Dec. 2004.

[Lin 1997] Y.-D. Lin, J.-W. Sheen, "Mode distinction and radiation efficiency analysis of planar leaky-wave source," IEEE Trans. Microw. Theory Tech., vol. 45, no.10, pp.1672-1680, November 1997.

[Liu 2002] L. Liu, C. Caloz, T. Itoh, "Dominant mode leaky-wave antenna with backfire-toendfire scanning capability," Electronics Letters, vol.38, no.23, pp.1414-1416, 7 Nov 2002

[Liu 2009] Z.G. Liu, Z.C. Ge and X.Y. Chen, "Research progress on Fabry-Perot resonator antenna," Journal of Zhejiang University SCIENCE A, Vol. 10, No. 4, 583-588, 2009.

[Llombart 2005] N. Llombart, A. Neto, G. Gerini, P. De Maagt, "Planar circularly EBG structures for reducing surface waves in printed antennas", IEEE Transactions on Antennas and Propagation, Vol. 53, no.10, pp. 3210-3218, Oct. 2005. 
[Llombart 2007] N. Llombart, A. Neto, G. Gerini, P. De Maagt, "1-D Scanning Arrays on Dense Dielectrics Using PCS-EBG Technology”, IEEE Transactions on Antennas and Propagation, Vol. 55, no.1, pp. 26-35, Jan. 2007.

[Losito 2007] O. Losito, "Design of conformal tapered leaky wave antenna," in Progress in Electromagnetics Research Symp., Aug. 2007, pp. 177-181.

[Lovat 2006-I] G. Lovat, P. Burghignoli and D. R. Jackson, "Fundamental properties and optimization of broadside radiation from uniform leaky-wave antennas," IEEE Trans. Antennas Propag., vol. 54, pp. 1442-1452, May 2006.

[Lovat 2006-II] G. Lovat, P. Burghignoli, F. Capolino, D.R. Jackson, "Bandwidth analysis of highly-directive planar radiators based on partially-reflecting surfaces," Antennas and Propagation, 2006. EuCAP 2006. First European Conference on, vol., no., pp.1, 6, 610 Nov. 2006

[Lovat 2006-III] G. Lovat, P. Burghignoli, F. Capolino, and D. R. Jackson, "High directivity in low-permittivity metamaterial slabs: Ray-optic vs. leaky-wave models," Microwave Opt. Techn. Lett., vol. 8, no. 12, pp. 2542-2548, Dec. 2006.

[Lovat 2006-IV] G. Lovat, P. Burghignoli, F. Capolino, D. R. Jackson, and D. R. Wilton, "Analysis of directive radiation from a line source in a metamaterials slab with low permittivity,” IEEE Trans. Antennas Propag., vol. 54, pp. 1017-1030, Mar. 2006.

[Lovat 2007] G. Lovat, P. Burghignoli and S. Celozzi "A tunable ferroelectric antenna for fixedfrequency scanning applications", IEEE Antennas Wireless Propag. Lett., vol. 5, pp. $353-3562006$

[Luukkonen 2008] O. Luukkonen, C.R. Simovski, A.V. Raisanen, S.A. Tretyakov, "An Efficient and Simple Analytical Model for Analysis of Propagation Properties in Impedance Waveguides," Microwave Theory and Techniques, IEEE Transactions on, vol.56, no.7, pp.1624,1632, July 2008

[Luukonen 2009] Olli Luukonen," Artificial Impedance Surfaces", Helsinki University of Technology, Supervisors: Antti Räisänen and Sergei Tretyakov, December 2009, available online: http://lib.tkk.fi/Diss/2009/isbn9789522482525/

[Luxey 2000] C. Luxey and J.-M. Laheurte, "Effect of reactive loading in microstrip leaky wave antennas," Electron. Lett., vol. 36, no. 15, pp. 1259-1260, Jul. 20, 2000.

[Machac 2013] J. Machac, M. Polivka, K. Zemlyakov, “A Dual Band Leaky Wave Antenna on a CRLH Substrate Integrated Waveguide," Antennas and Propagation, IEEE Transactions on, vol.61, no.7, pp.3876, 3879, July 2013 
[Maci 2005] S. Maci, M. Caiazzo, A. Cucini, and M. Casaletti, "A pole-zero matching method for EBG surfaces composed of a dipole FSS printed on a grounded dielectric slab," IEEE Trans. Antennas Propag., vol. 53, no. 1, pp. 70-81, Jan. 2005.

[Maci 2011] S. Maci, G. Minatti, M. Casaletti, M. Bosiljevac, "Metasurfing: Addressing Waves on Impenetrable Metasurfaces," Antennas and Wireless Propagation Letters, IEEE, vol.10, no., pp.1499, 1502, 2011

[Maheri 1988] H. Maheri, M. Tsutsumi, and N. Kumagi, "Experimental studies of magnetically scannable leaky-wave antennas having a corrugated ferrite slab/dielectric layer structure," IEEE Trans. Antennas Propagat., vol. 36, pp. 911-917, Nov. 1988.

[Maloney 2000] J.C. Maloney, M.P. Kesler, L.M. Lust, L.N. Pringle, T.L. Fountain, P.H. Harms, and G.S. Smith, "Switched fragmented aperture antennas," in Antennas and Propagation Society International Symposium, 2000. IEEE, pages 310-313 vol.1, 2000.

[Manasson 1995] V. A. Manasson, L. S. Sadovnik, A. Moussessian, and D. B. Rutledge, "Millimeter-wave diffraction by a photo-induced plasma grating," IEEE Trans. Microwave Theory Tech., vol. 43, pp. 2288-2290, Sept. 1995.

[Manasson 2010] V. A. Manasson et al, "Electronically reconfigurable aperture (ERA): A new approach for beam-steering technology," Phased Array Systems and Technology (ARRAY), 2010 IEEE International Symposium on, vol., no., pp.673-679, 12-15 Oct. 2010

[Marcuvitz 1956] N. Marcuvitz, "On field representations in terms of leaky modes or Eigenmodes," Antennas and Propagation, IRE Transactions on, vol.4, no.3, pp.192, 194, July 1956

[Martinez-Ros 2012-I] A. J. Martinez-Ros, J.L. Gomez-Tornero, and G. Goussetis, "Planar leaky-wave antenna with flexible control of the complex propagation constant," IEEE Trans. Antennas Propag., vol. 60, no. 3, pp. 1625 -1630, Mar. 2012.

[Martínez-Ros 2012-II] A.J. Martinez-Ros, J.L. Gómez-Tornero, and G. Goussetis, "Broadside radiation from radial arrays of substrate integrated Leaky-Wave antennas," in 6th European Conf. on Antennas and Propagation (EuCAP 2012), Prague, Czech Republic, Mar. 2012.

[Martínez-Ros 2013-I] A.J. Martínez-Ros, J.L Gomez-Tornero, G. Goussetis, "Holographic Pattern Synthesis With Modulated Substrate Integrated Waveguide Line-Source LeakyWave Antennas," Antennas and Propagation, IEEE Transactions on , vol.61, no.7, pp.3466,3474, July 2013 
[Martinez-Ros 2013-II] A.J. Martínez-Ros, J.L Gomez-Tornero, F.J. Clemente-Fernandez, J. Monzo-Cabrera, "Microwave Near-Field Focusing Properties of Width-Tapered Microstrip Leaky-Wave Antenna," Antennas and Propagation, IEEE Transactions on , vol.61, no.6, pp.2981,2990, June 2013

[Mateo-Segura 2011] C. Mateo-Segura, G. Goussetis, A.P. Feresidis, "Sub-Wavelength Profile 2-D Leaky-Wave Antennas With Two Periodic Layers," Antennas and Propagation, IEEE Transactions on, vol.59, no.2, pp.416, 424, Feb. 2011

[Mateo-Segura 2012] C. Mateo-Segura, M. Garcia-Vigueras, G. Goussetis, A.P. Feresidis and J.L Gomez-Tornero, "A Simple Technique for the Dispersion Analysis of Fabry-Perot Cavity Leaky-Wave Antennas," Antennas and Propagation, IEEE Transactions on , vol.60, no.2, pp.803,810, Feb. 2012

[Mateo-Segura 2014] C. Mateo-Segura, A.P. Feresidis, G. Goussetis, "Bandwidth Enhancement of 2-D Leaky-Wave Antennas With Double-Layer Periodic Surfaces," Antennas and Propagation, IEEE Transactions on, vol.62, no.2, pp.586,593, Feb. 2014

[Matlab 2012] MATLAB 2010b, The MathWorks, Inc., Natick, Massachusetts, United States.

[Matsuzawa 2005] S. Matsuzawa, K. Sato, Y. Inoue, T. Nomura, "Beam steering left-handed leak wave antenna for millimeter-wave applications". Proc. IEEE Antennas Propag. Soc. Int Symp., pp. 709-712, 2005

[Matsuzawa 2006] S. Matsuzawa, Y. Inoue, K. Sato, T. Nomura: JP211328A (2006).

[Matsuzawa 2007] S. Matsuzawa, K. Sato, T. Nomura, Y. Inoue: JP201571A (2007).

[Medina 2010] F. Medina, F. Mesa, and D. C. Skigin, "Extraordinary transmission through arrays of slits: a circuit theory model," IEEE Trans. Microwave Theory Tech., vol. 58, no. 1, pp. 105-115, Jan. 2010.

[Menzel 1979] W. Menzel, "A new travelling wave antenna in microstrip”, Arch. Elektron. Uebertrag. Tech., vol. 33, no. 4, pp. 137-140, Apr. 1979.

[Mias 2007] C. Mias, and J.H. Yap, "A varactor-tuneable high impedance surface with a resistive-lumped-element biasing grid," IEEE Trans. Antennas Propag, vol. 55, no. 7, pp. 1955-1962, July 2007.

[Minatti 2011] G. Minatti, F. Caminita, M. Casaletti, S. Maci, "Spiral leaky-wave antennas based on modulated surface impedance," IEEE Trans. Antennas Propagat., vol. 59, no. 12, pp. 4436 - 4444, Nov. 2011.

[Mittra 1988] R. Mittra, C. H. Chan, T. Cwik "Techniques for analyzing frequency selective surfaces-a review," Proceedings of the IEEE, vol.76, no.12, pp.1593-1615, Dec 1988. 
[Moghadas 2013] H. Moghadas, M. Daneshmand, P. Mousavi, "Single-layer partially reflective surface for an orthogonally-polarised dual-band high-gain resonant cavity antenna," Microwaves, Antennas \& Propagation, IET, vol.7, no.8, pp.656-662, June 52013

[Monnai 2011] Y. Monnai, H. Shinoda, "Focus-Scanning Leaky-Wave Antenna With Electronically Pattern-Tunable Scatterers," Antennas and Propagation, IEEE Transactions on, vol.59, no.6, pp.2070,2077, June 2011

[Munk 2000] B. A. Munk, “Frequency Selective Surfaces: Theory and Design”, John Wiley and Sons, 2000.

[Neto 2003] A. Neto, S. Bruni, G. Gerini, M. Sabbadini, "Leaky wave lens antenna for wide/multi band application," Wireless Technology, 2005. The European Conference on, vol., no., pp.439, 442, 3-4 Oct. 2005

[Noujeim 1998] K. M. Noujeim, "Fixed-frequency beam-steerable leaky-wave antennas.” Ph.D. Thesis. Universitv of Toronto. Ontario, Canada, 1998.

[Noujeim 2003] K.M. Noujeim, "Wave propagation characteristics of a reactively loaded microstrip," IEEE MTT-S Int. Microwave Symposium Digest, vol.2, pp. 821 - 824, June 2003.

[Neto 2006] A. Neto, N. Llombart, G. Gerini, P. De Maagt, "On the optimal radiation bandwidth of printed slot antennas surrounded by EBGs", IEEE Transactions on Antennas and Propagation, Vol. 54, no.4, pp. 1074-1083, Apr. 2006.

[Nguyen 2007] H.V. Nguyen, S. Abielmona, A. Rennings, C. Caloz, "Pencil-Beam Full-Space Scanning 2D CRLH Leaky-Wave Antenna Array," Signals, Systems and Electronics, 2007. ISSSE '07. International Symposium on, vol., no., pp.139, 142, July 30 2007-Aug. 22007

[Nguyen 2011] H.V. Nguyen, S. Abielmona, and C. Caloz, "Performance-enhanced and symmetric full-space scanning end-switched CRLH LWA," IEEE Antennas Wireless Propag. Lett. vol.10, pp.709-712, 2011.

[Oliner 1959] A. Oliner and A. Hessel, "Guided waves on sinusoidally-modulated reactance surfaces," IRE Trans. Antennas Propag., vol. AP-7, no. 5, pp. 201-208, Dec. 1959.

[Oliner 1979] A.A. Oliner and S. T. Peng, "A New Class of Leaky Modes on Open Dielectric Waveguides," 1979, pp. 569-571.

[Oliner 1985] A.A. Oliner, S. T. Peng, and K. M. Shang, "Leakage from a gap in NRD guide," in Proc. IEEE MTT Int. Microwave Symp. Dig., 1985, pp. 619-622. 
[Oliner 1986-I] A.A. Oliner and K. S. Lee, "The Nature of the Leakage from Higher Modes on Microstrip Line," IEEE MTT-S International Microwave Symposium Digest 1986, pp.57-60, 2-4 June 1986

[Oliner 1986-I] A.A. Oliner and K. S. Lee, "The nature of the leakage from higher modes on microstrip line, ” in Int. Microwave Symp. Dig., MTT-S, vol.86, pp. 57-60, Jun. 1986.

[Oliner 1986-II] A.A. Oliner and K. S. Lee, "Microstrip leaky wave strip antennas," in IEEE AP-S Int. Symp. Dig., Philadelphia, PA, pp. 443-446, Jun.1986.

[Oliner 1987] A.A. Oliner, "Leakage from higher modes on microstrip line with application to antennas," Radio Sci., vol. 22, no. 6, pp. 907-912, Nov. 1987.

[Oliner 1988] A.A. Oliner, "Scannable Millimeter Wave Arrays," Final Report on RADC Contract No. F19628-84-K-0025, Polytechnic University, September 30, 1988, 528 pages in two volumes.

[Oliner 1990] A.A. Oliner, "A new class of scannable millimeter wave antennas," in Proc. 20th European Microwave Conf., Budapest, Hungary, Sept. 1990, pp. 95-104.

[Oliner 2007] A.A. Oliner and D.R. Jackson, "Leaky-Wave Antennas", in Antenna Engineering Handbook, 4th ed., J. L. Volakis (Ed.), McGraw-Hill, New York, , 2007, Chap. 11.

[O’Nians 1966] F. O'Nians and J. Matson, “Antenna feed system utilizing polarization independent frequency selective intermediate reflector," U.S. Patent 3-231-892, Jan. 1966.

[Orr 2014] R. Orr, G. Goussetis, V. Fusco "Design Method for Circularly Polarized FabryPerot Cavity Antennas", Antennas and Propagation, IEEE Transactions on, vol.62, no.1, pp.19-26, Jan. 2014

[Ouegraogo 2011] R. Ouegraogo, E. Rothwell, and B. Greetis, "A reconfigurable microstrip leaky-wave antenna with a broadly steerable beam," IEEE Trans. Antennas Propag., vol.59, no.8, pp. 3080-3083, Aug. 2011.

[Ourir 2007] A. Ourir, S.N. Burokur, A. de Lustrac, "Electronically reconfigurable metamaterial for compact directive cavity antennas," Electronics Letters, vol.43, no.13, pp.698-700, June 212007.

[Ourir 2009] A. Ourir, Burokur, S.N., Yahiaoui, R. \& de Lustrac, A. (2009). Directive metamaterial-based subwavelength resonant cavity antennas - Applications for beam steering. C. R. Physique, Vol.10, No.5, pp. 414-422, ISSN 1631-0705, June 2009.

[Palmer 2011] K. M. Palmer "Intellectual ventures invents beam-steering metamaterials antenna IV and others aim at cheap in-flight broadband," IEEE Spectrum Magazine, Nov. 2011. 
[Panagamuwa 2006] C. J. Panagamuwa, A. Chauraya, and J. C. Vardaxoglou, "Frequency and beam reconfigurable antenna using photoconductive switches", IEEE Trans. Antennas Propag.,vol. 54, no. 2, pp. 449-454, Feb. 2006.

[Peng 1978] S.T. Peng and F. Schwering, "Dielecric Grating Antennas," R\&D Tech. Rep., COADCOM-78-3, Fort Monmouth, NJ, July 1978.

[Perez-Palomino 2012] G. Perez-Palomino, J.A. Encinar, M. Barba, E. Carrasco, "Design and evaluation of multi-resonant unit cells based on liquid crystals for reconfigurable reflectarrays," Microwaves, Antennas \& Propagation, IET , vol.6, no.3, pp.348-354, February 212012.

[Perruisseau-Carrier 2008] J. Perruisseau-Carrier and A. K. Skrivervik, "Monolithic MEMSbased reflectarray cell digitally reconfigurable over a $360^{\circ}$ phase range," IEEE Antennas Wireless Propagat. Lett., vol. 7, pp. 138-141, 2008.

[Perruisseau-Carrier 2010-I] J. Perruisseau-Carrier, "Versatile reconfiguration of radiation patterns, frequency and polarization: a discussion on the potential of controllable reflectarrays for software-defined and cognitive radio systems," in Proceedings of the IEEE International Microwave Workshop Series on RF Front-Ends for Software Defined and Cognitive Radio Solutions (IMWS '10), pp. 1-4,Aviero, Portugal, February 2010.

[Perruisseau-Carrier 2010-II] J. Perruisseau-Carrier, "Dual-Polarized and Polarization-Flexible Reflective Cells with Dynamic Phase Control," IEEE Trans. Antennas Propag., Volume.58, Issue.5, pp.1494, 2010.

[Piazza 2010] D. Piazza, M. D'Amico, "Pattern and polarization reconfigurable CRLH leaky wave antenna," Antennas and Propagation (EuCAP), 2010 Proceedings of the Fourth European Conference on, vol., no., pp.1,5, 12-16 April 2010

[Podilchak 2010] S.K. Podilchak, Al.P. Freundorfer, Y.M.M. Antar, "A new configuration of printed non-directive surface-wave sources for advanced beam control," Antenna Technology and Applied Electromagnetics \& the American Electromagnetics Conference (ANTEM-AMEREM), 2010 14th International Symposium on , vol., no., pp. 1,4, 5-8 July 2010

[Pozar 2005] D. M. Pozar. Microwave Engineering, 3rd ed. Wiley, Hoboken, NJ, 2005.

[Pringle 2004] Pringle, L.N. et al, "A reconfigurable aperture antenna based on switched links between electrically small metallic patches," Antennas and Propagation, IEEE Transactions on, vol.52, no.6, pp. 1434-1445, June 2004 
[Raisanen 2012] A.V. Raisanen et al, "Antennas for electronic beam steering and focusing at millimeter wavelengths," Electromagnetics in Advanced Applications (ICEAA), 2012 International Conference on , vol., no., pp.1235,1237, 2-7 Sept. 2012

[Riel 2007] M. Riel and J. J. Laurin, "Design of an electronically-beam scanning reflectarray using aperture-coupled elements", IEEE Trans. Antennas Propag., vol. 55, no. 5, pp.1260 -1266 2007

[Rock 2008] J.C. Rock, J.H. Mullins, J.P. Booth, T. Hudson, "The Past, Present, and Future of Electronically-Steerable Phased Arrays in Defense Applications," Aerospace Conference, 2008 IEEE, vol., no., pp.1,7, 1-8 March 2008

[Rodrigo 2010] D. Rodrigo, Y. Damgaci, N. Biyikli, B. A. Cetiner, J. Romeu, and L. Jofre, "Mems-reconfigurable antenna based on a multi-size pixelled geometry," in Proc. Fourth European Conf. Antennas and Propagation (EuCAP), pp. 1-4, 2010

[Rodrigo 2011] D. Rodrigo, Y. Damgaci, M. Unlu, B.A. Cetiner, J. Romeu, L. Jofre, “Antenna Reconfigurability based on a Novel parasitic pixel layer," Antennas and Propagation (EUCAP), Proceedings of the 5th European Conference on, vol., no., pp.3497-3500, 11-15 April 2011

[Rodrigo 2012] D. Rodrigo, L. Jofre, "Frequency and Radiation Pattern Reconfigurability of a Multi-Size Pixel Antenna", Antennas and Propagation, IEEE Transactions on, On page(s): 2219 - 2225 Volume: 60, Issue: 5, May 2012

[Rodrigo 2013] D. Rodrigo, L. Jofre, J. Perruisseau-Carrier, "Unit Cell for Frequency-Tunable Beamscanning Reflectarrays," Antennas and Propagation, IEEE Transactions on, vol.61, no.12, pp.5992-5999, Dec. 2013

[Roig 2013] M. Roig M. Maasch, C. Damm, R. Jakoby, "Steerable Ka-Band leaky wave antenna based on liquid crystal material", Advanced Electromagnetic Materials in Microwaves and Optics (METAMATERIALS), 2013 7th International Congress on, On page(s): 540 - 545

[Romanofsky 2006] R. Romanofsky, "Special issues and features of a scanning reflectarray antenna based on ferroelectric thin film phase shifters," in 1st European Conference on Antennas and Propagation (EuCAP 2006), Nice, France, 2006.

[Rotman 1959] W. Rotman and A.A. Oliner, "Asymmetrical trough waveguide antenna, IRE Trans. Antennas Propag., Vol. 7, pp. 153-162, Aril 1959

[Rusch 2013] C. Rusch, S. Beer, P. Pahl, T. Zwick, "Multilayer holographic antenna with beam scanning in two dimensions at W-band," Antennas and Propagation (EuCAP), 2013 7th European Conference on, vol., no., pp.2625, 2628, 8-12 April 2013 
[Rutschlin 2013] M. Rutschlin, V. Sokol, "Reconfigurable Antenna Simulation: Design of Reconfigurable Antennas with Electromagnetic Simulation," Microwave Magazine, IEEE, vol.14, no.7, pp.92, 101, Nov.-Dec. 2013

[Sazegar 2012] M. Sazegar, Z. Yuliang, C. Kohler, H. Maune, M. Nikfalazar, J.R. Binder, R. Jakoby, "Beam Steering Transmitarray Using Tunable Frequency Selective Surface With Integrated Ferroelectric Varactors," Antennas and Propagation, IEEE Transactions on, vol.60, no.12, pp.5690,5699, Dec. 2012

[Shumpert 1999] J.A. Shumpert, W.J. Chappell, L.P.B. Katehi, "Parallel-plate mode reduction in conductor-backed slots using electromagnetic bandgap substrates," IEEE Trans. Microw. Theory Tech., vol.47, no.11, pp.2099-2104, Nov 1999.

[Sievenpiper 1999] D.F. Sievenpiper, L. Zhang, F. J. Broas, N. G. Alexopulos, and E. Yablonovitch, "High-impedance electromagnetic surfaces with a forbidden frequency band," IEEE Trans. Microw. Theory Tech., vol. 47, no. 11, pp. 2059-2074, Nov. 1999.

[Sievenpiper 2002] D. Sievenpiper and J. Schaffner, "Beam steering microwave reflector based on electrically tuneable impedance surface," Electron. Lett., vol. 38, no. 21, pp. 12371238, Oct. 2002.

[Sievenpiper 2003] D. F. Sievenpiper, J. H. Schaffner, H. J. Song, R. Y. Loo, and G. Tangonan, "Two-Dimensional beam steering using an electrically tuneable impedance surface," IEEE Trans. Antennas Propag., vol. 51, no. 10, pp. 2713-2722, Oct. 2003.

[Sievenpiper 2005] D.F. Sievenpiper, "Forward and backward leaky wave radiation with large effective aperture from an electronically tuneable textured surface," IEEE Trans. Antennas Propag., vol. 53, no. 1, pp. 236-247 Jan. 2005.

[Sievenpiper 2005] D.F. Sievenpiper, "Superluminal Waveguides Based on Non-Foster Circuits for Broadband Leaky-Wave Antennas," Antennas and Wireless Propagation Letters, IEEE, vol.10, no., pp.231,234, 2011

[Sterner 2009] M. Sterner, D. Chicherin, A. V. Raisenen, G. Stemme, and J. Oberhammer, "RF MEMS high-impedance tuneable metamaterials for millimeter-wave beam steering," in Proc. 22nd Int. Conf. Micro Electro Mech. Sys., Sorrento, 2009, pp. 896-899.

[Suntives 2011] A. Suntives, and S.V. Hum, "An electronically tuneable half-mode substrate integrated waveguide leaky-wave antenna," Antennas and Propagation (EUCAP), Proceedings of the 5th European Conference on, pp. 3670-3674, April 2011.

[Suntives 2012] A. Suntives, and S.V. Hum, "A Fixed-Frequency Beam-Steerable Half-Mode Substrate Integrated Waveguide Leaky-Wave Antenna," Antennas and Propagation, IEEE Transactions on, vol.60, no.5, pp.2540,2544, May 2012. 
[Sutinjo 2008] A. Sutinjo, M. Okoniewski and R.H. Johnston, R.H., "Beam-Splitting Condition in a Broadside Symmetric Leaky-Wave Antenna of Finite Length," IEEE Antennas Wireless Propag. Lett., vol.7, no., pp.609-612, 2008

[Tamir 1963-I] T. Tamir and A.A. Oliner, "Guided complex waves. Part 1: Fields at an interface," Proc. Inst. Elec. Eng., Vol. 110, pp. 310-324, no. 2, Feb. 1963

[Tamir 1963-II] T. Tamir, A.A. Oliner, "Guided Complex Waves, Part II: Relation to Radiation Patterns," Proc. Inst. Elec. Eng., Vol. 110, pp. 325-334, no. 2, Feb. 1963.

[Tamir 1969] T. Tamir, Antenna Theory, Part II, R. E. Collin and R. F. Zucker, Eds. New York: McGraw-Hill, 1969, chap. 20.

[Taylor 2003-I] G. C. Taylor, et. al., "Reconfigurable Antenna”, U. S. Patent no.: US 6,567,046 B2, May 20, 2003.

[Taylor 2003-II] G. C. Taylor, A. Rosen, A. E. Fathy, P. K. Swain, and S. M. Perlow, "Surface PIN device", U. S. Patent no.: US 6,617,670 B2, Sep., 9, 2003.

[Tawk 2011] Y. Tawk, J. Costantine, K. Avery, and C. G. Christodoulou, "Implementation of a cognitive radio front-end using rotatable controlled reconfigurable antennas," IEEE Trans. Antennas Propag., vol. 59, no. 5, pp. 1773-1778, May 2011.

[Tawk 2012] Y. Tawk, J. Costantine, and C. G. Christodoulou, "A varactor based reconfigurable filtenna," IEEE Antennas and Wireless Propagation Letters, vol. 11, pp. 716-719, 2012.

[Temelkuaran 2000] B. Temelkuaran, M. Bayindir, E. Ozbay, R. Biswas, M. M. Sigalas, G. Tuttle, and K. M. Ho, "Photonic crystal-based resonant antenna with a very high directivity," J. Appl. Phys., vol. 87, no. 1, pp. 603-605, Jan. 2000.

[Topalli 2009] K. Topalli, E. Erdil, O. A. Civi, S. Demir, S. Koc, and T. Akin, “Tunable dualfrequency RF MEMS rectangular slot ring antenna," Sensors and Actuators A, vol. 156, no. 2, pp. 373-380, 2009.

[Trentini 1956] G.V. Trentini, "Partially reflecting sheet arrays," Antennas and Propagation, IRE Transactions on, vol.4, no.4, pp.666,671, October 1956.

[Tretyakov 2003-I] S. Tretyakov, "Analytical Modelling in Applied Electromagnetics”. Boston, MA: Artech House, 2003.

[Tretyakov 2003-II] S.A. Tretyakov and S I. Maslovski, "Thin absorbing structure for all incidence angles based on the use of a high-impedance surface," Microw.Opt. Technol. Lett., vol. 38, no. 3, pp. 175-178, Aug. 2003 
[Varadan 1994] V.K. Varadan, V.V. Varadan, K.A. Jose, and J.F. Kelly, "Electronically steerable leaky wave antenna using a tuneable ferroelectric material," Smart Mater. Struct., vol. 3, pp. 470-475, June 1994.

[Veselago 1968] V.G. Veselago, "The electrodynamics of substances with simultaneously negative values of $\varepsilon$ and $\mu$ ", Soviet Physical Uspekhi, vol.10, no.6, pp. 510-514. Jan. 1968.

[Walter 1965] C. H. Walter, “Traveling Wave Antennas”, McGraw-Hill, New York, 1965.

[Washington 2002] F. Washington, H.-S. Yoon, M. Angelino, and W.H. Theunissen, "Design, modeling, and optimization of mechanically reconfigurable aperture antennas," IEEE Transactions on Antennas and Propagation, vol. 50, pp. 628-637, May 2002.

[Wang 1999] C. J. Wang, C. F. Jou, and J. J. Wu, "A Novel two-beam scanning leaky-wave antenna", IEEE Trans. Antennas Propagat., vol. 47, pp.1314 -1317 1999

[Wang 2000] C. J. Wang, C. F. Jou, and Y. C. Shih, "A beam-switchable scanning leaky-wave antenna", Electron. Lett., vol. 36, no. 7, pp.596 -597 2000

[Wang 2014] M. Wang, C. Huang, P. Chen, Y. Wang, Z. Zhao, X. Luo, "Controlling Beamwidth of Antenna Using Frequency Selective Surface Superstrate," Antennas and Wireless Propagation Letters, IEEE, vol.13, no., pp.213,216, 2014

[Weedon 2001] W. H. Weedon, W. J. Payne, and G. M. Rebeiz, "MEMS switched reconfigurable antennas," in Proceedings of the IEEE International Symposium on Antennas and Propagation, pp. 654-657, 2001.

[Weily 2005-I] A.R. Weily, K. P. Esselle, B. C. Sanders, and T. S. Bird, "High-gain 1-D EBG resonator antenna," Microw. Opt. Technol. Lett., vol. 47, no. 2, pp. 107-114, 2005.

[Weily 2005-II] A.R. Weily, L. Horvath, K. P. Esselle, B. C. Sanders, and T. S. Bird, "A planar resonator antenna based on a woodpile EBG material," IEEE Trans. Antennas Propag., vol. 53, no. 1, pp. 216-223, Jan. 2005.

[Weily 2006] A.R. Weily, K. P. Esselle, T. S. Bird, and B. C. Sanders, "High gain circularly polarized 1-D EBG resonator antenna," Electron. Lett., vol. 42, no. 18, pp. 1012-1013, Aug. 2006.

[Weily 2007-I] A.R. Weily, K. P. Esselle, T. S. Bird, and B. C. Sanders, "Dual resonator 1-D EBG antenna with slot array feed for improved radiation bandwidth," IET Microw., Antennas Propag., vol. 1, no. 1, pp. 198-203, Feb. 2007.

[Weily 2007-II] A.R. Weily, T. S. Bird, and Y. J. Guo, "A reconfigurable high gain EBG antenna," in Proc. 2007 URSI EMT-Symp., Ottawa, Canada, 2007. 
[Weily 2008] A.R. Weily, T.S. Bird, and Y.J. Guo, "A reconfigurable high-gain partially reflecting surface antenna," IEEE Trans. Antennas Propagat., vol. 56, no. 11, pp. 33823390, Nov. 2008.

[White 2009] C. R. White and G. M. Rebeiz, "Single- and dual-polarized tunable slot-ring antennas," IEEE Transactions on Antennas and Propagation, vol. 57, no. 1, pp. 19-26, 2009.

[Xu 2005] F. Xu and K. Wu, "Guided-wave and leakage characteristics of substrate integrated waveguide,” IEEE Trans. Microw. Theory Tech., vol. 53, no. 1, pp. 66-73, Jan. 2005.

[Yang 2004] X. S. Yang, B. Y. Wang, Y. Zhang, "Pattern-reconfigurable quasi-Yagi microstrip antenna using a photonic band-gap structure," Microwave and Optical Technology Letters, vol. 42, no. 4, pp. 296-297, Aug. 2004.

[Yashchyshyn 2005-I] Y. Yashchyshyn, and J. Modelski, "Rigorous analysis and investigations of the scan antennas on a ferroelectric substrate," IEEE Trans. Microwave Theory Tech., vol. 53, no. 2, pp. 427-438, Feb. 2005.

[Yashchyshyn 2005-II] Y. Yashchyshyn, and J. Modelski, "A reconfigurable leaky-wave microstrip antenna," 2005 European Microwave Conference, vol. 1, pp. 4, 2005.

[Yashchyshyn 2009] Y. Yashchyshyn, et al, "Development and Investigation of an Antenna System With Reconfigurable Aperture," Antennas and Propagation, IEEE Transactions on, vol.57, no.1, pp.2,8, Jan. 2009

[Yashchyshyn 2010-I] Y. Yashchyshyn, J. Marczewski, D. Tomaszewski, "Investigation of the S-PIN Diodes for Silicon Monolithic Antennas With Reconfigurable Aperture," Microwave Theory and Techniques, IEEE Transactions on, vol.58, no.5, pp.1100, 1106, May 2010

[Yashchyshyn 2010-II] Y. Yashchyshyn, "Reconfigurable Antennas: The State of the Art," Microwave Radar and Wireless Communications (MIKON), 2010 18th International Conference on , vol., no., pp.1,9, 14-16 June 2010

[Yuehe 2012] G. Yuehe, K.P. Esselle, T.S. Bird, "The Use of Simple Thin Partially Reflective Surfaces With Positive Reflection Phase Gradients to Design Wideband, Low-Profile EBG Resonator Antennas," Antennas and Propagation, IEEE Transactions on , vol.60, no.2, pp.743,750, Feb. 2012

[Zvolensky 2010] T. Zvolensky, D. Chicherin, A.V. Räisänen, C. Simovski, "Leaky-wave antenna based on microelectromechanical systems-loaded microstrip line," Microwaves, Antennas \& Propagation, IET , vol.5, no.3, pp.357,363, Feb. 212011 
[Zhao 2004] T. Zhao, D. R. Jackson, J. T. Williams, and A.A. Oliner, "Simple CAD model for a dielectric leaky-wave antenna," IEEE Antennas Wireless Propag. Lett., vol. 3, pp. 243345, Dec. 2004.

[Zhao 2005-I] T. Zhao, D. R. Jackson, J. T. Williams, and A.A. Oliner, "General formulas for 2D leaky-wave antennas," IEEE Trans. Antennas Propagat., vol. 53, No.11, pp.35253533, Nov. 2005.

[Zhao 2005-II] T. Zhao, D.R Jackson, J.T. Williams, H.-Y.D Yang, A.A Oliner, "2-D periodic leaky-wave antennas-part I: metal patch design,” IEEE Trans. Antennas Propag, Vol. 53, No 11, pp. 3505-3514, Nov. 2005.

[Zhang 2010] Y. Li, Z. Zhang, W. Chen, and Z. Feng, "Polarization reconfigurable slot antenna with a novel compact CPW-to-slotline transition for WLAN application," IEEE Antennas and Wireless Propagation Letters, vol. 9, pp. 252-255, 2010.

[Zhao 2005-III] T. Zhao, D. R. Jackson and J. T. Williams, "2-D periodic leaky-wave antennas Part II: Slot design,” IEEE Trans. Antennas Propag., vol. 53, no. 11, pp. 3515-3524, Nov. 2005.

[Zuliani 2004] M. Zuliani, A. Petosa, A. Ittipiboon, L. Roy, and R. Chaharmir, "Microstrip periodic leaky-wave antenna with optical control and beam scanning capabilities," IEEE Antennas and Propagat. Society Int. Symposium, vol. 2, pp. 1831 - 1834, 20-25 June 2004. 


\section{Framework of the Research}

The development of this thesis has been supervised by Dr. José Luis Gómez Tornero and supported by the research Group of Electromagnetism Applied to Telecommunications (Grupo de Electromagnetismo Aplicado a las Telecomunicaciones, GEAT), in the department of Information and Communications Technologies (ICT) of Universidad Politécnica de Cartagena (UPCT).

This thesis has been carried out within the doctoral program "Information and Communications Technologies", awarded "Mention of Excellence" by the Spanish Ministry of Education and Science (“Ministerio de Educación y Ciencia”) since 2011.

In addition, this thesis has been developed in collaboration with two prestigious institutions. On one hand, the manufacturing and tests of the experimental prototypes fabricated for the development of the thesis have been performed in collaboration with the Information and Communications Technologies Centre of the Commonwealth Scientific and Industrial Research Organisation, (CSIRO ICT), in Sydney (Australia). This process was funded by CSIRO ICT centre and supervised by Dr. Andrew R. Weily and Prof. Y. Jay Guo. Also, the author performed a three-month research stay at the Institute of Electronics, Communications and Information Technology (ECIT) of Queen's University of Belfast (QUB), in Northern Ireland (United Kingdom), where it was developed part of the analysis and design of the new electronically reconfigurable leaky wave antennas presented in this thesis. This stay was possible thanks to Dr. George Goussetis and Prof. Vincent Fusco.

Likewise, the work of this thesis has been performed within the framework of the following national research projects: TEC2007-67630-C03-02/TCM, from the Spanish Ministry of Industry, Tourism and Commerce (Ministerio de Industria, Turismo y Comercio), TEC201021520-C04-04, from the Ministry of Science and Innovation (Ministerio de Ciencia $e$ Innovación) and European FEDER (Fondo Europeo de Desarrollo Regional) and 08833/PI/08, from Spanish Seneca Foundation of Murcia (Fundación Séneca de la Región de Murcia).

The three months research stage at Queen's University of Belfast was funded by Universidad Politécnica de Cartagena (student mobility program ref. PMPDI-UPCT-2012).

The author wants to express his gratitude to all the institutions which have supported the work developed in this thesis. 


\section{Acknowledgements}

Sin duda, la realización de esta tesis ha sido una de las experiencias más intensas y enriquecedoras de mi vida tanto a nivel personal como profesional. A lo largo de este viaje, muchas son las personas que me han apoyado y han contribuido directa o indirectamente al resultado final de este proyecto. Por esta razón, a continuación deseo dedicarles unas palabras, porque sin todos y cada uno de ellos, este camino nunca hubiera sido tan gratificante.

Deseo comenzar por José Luis por su labor como director. Nada de este trabajo hubiera sido lo mismo sin haber contado con su supervisión y experiencia. Gracias por todo el tiempo dedicado, tu confianza en mí, la ilusión y el esfuerzo compartidos y todo lo aprendido, no sólo a nivel técnico, sino también personal. La ilusión y vitalidad que transmites me acompañarán siempre.

A Alejandro M. Sala, al grupo de Investigación Telemática y a mis tutores de la empresa DH, Paco y Mariano, por darme la oportunidad de trabajar con ellos a la vez que trabajaba a tiempo parcial en esta tesis. Sin lugar a dudas, esos tres años de mi vida han sido también una experiencia repleta de ilusiones y buenos momentos compartidos que recordaré con gran cariño. Además ha sido algo doblemente enriquecedor para mí, puesto que me ha permitido vivir la investigación desde un punto de vista más aplicado y no sólo académico, como suele ocurrir. En especial deseo agradecerle a Alejandro su confianza, apoyo y ánimo perpetuos, siempre facilitándome el camino de esfuerzo que supone el dedicarse en profundad a diferentes temas de investigación a la vez. Pero sobretodo deseo agradecerle la oportunidad que me brindó para dedicarme a una de las cosas que más me gustan, la investigación. A Paco y Mariano también deseo agradecerles su confianza y apoyo y a enseñarme a ver siempre oportunidades en situaciones donde sólo parecía haber problemas. También deseo agradecerle al grupo de Telemática su acogida durante esos años, y en especial a Joan sus consejos y revisiones, siempre muy apreciados, y por ser un ejemplo en la enseñanza y la investigación de nuestra universidad.

Deseo agradecerle a mi grupo, el GEAT, recibirme con los brazos abiertos y darme su respaldo y apoyo. Resaltar a Alejandro A. Melcón y Fernando por hacerme siempre el camino un poco más fácil cuando lo he necesitado. También a mis compañeros de laboratorio: Alejandro M., Attieh, David Cañete, Juan Pascual, José, María, Mónica, Sebas, Jorge G. y Jorge M. Sin vosotros ni las largas horas de trabajo, ni los congresos hubieran sido tan gratificantes y divertidos.

No quiero olvidarme de agradecer a la ETSIT y todos sus profesores todo lo aprendido durante la carrera, que ha sido crucial en estos años de mi vida, y al personal de la UPCT por vuestra 
profesionalidad. En especial a Ambrosio, de reprografía, y a Trini y Marisa, de Doctorado, por el cariño con el que me habéis recibido siempre y por la gran calidad humana que tenéis.

This thesis would have never been the same without the collaboration of my Australian fellows Andrew and Jay, from CSIRO ICT. I feel really fortunate to have worked with them during these years. Thank you very much for the countless emails and reports, the technical advices and the strong and continuous support. Thanks to you, this long journey has been much more rewarding.

To George Goussetis, Vincent Fusco, and the Queen's University of Belfast, for giving me the opportunity to perform a research stay in Belfast. Also remember the good people and friends I had the pleasure to meet there: Efstratios, Dmitry, Farhan, Gwendal, Keissy, Pak, Peter, Rob...Thank you very much for making my stay so nice and unforgettable.

También quiero agradecerles y dedicarles parte de esta tesis a todos mis amigos por animarme y haber estado siempre ahí. En particular a Ángel, Richi, Emilio, Cavas, Luis y Sergio por vuestro interés en mi trabajo y los buenos ratos que siempre pasamos juntos. También a mis colegas salseros por todos esos bailes. A los de patinaje por esas rutas de última hora. A mis camaradas del "Desastre", Jesús y José Luis por esas regatas inolvidables. Por último, a mis excompañeros de la empresa DH, a los que tuve la suerte de conocer y en especial a Antonio M. R., por su bondad inigualable y los grandes momentos que pasamos trabajando juntos, y a Agustín, por esas bajadas inolvidables desde el Veleta.

Deseo agradecer a mi familia su cariño y hacerme esto infinitamente más fácil, a mi abuelo Juan, por su buen humor y ser un ejemplo para mí, y por último a mis padres, Cari e Isidoro, a mi hermana Sara y a mi pareja Cristina, por su paciencia, apoyo incondicional y llenar mi vida de sentido. A vosotros os debo gran parte de lo que soy, y por ello os dedico esta tesis con todo mi cariño. 